ARTICLE TYPE

\title{
Supramolecular catalysis: Part 1: non-covalent interactions as a tool for building and modifying homogeneous catalysts
}

\author{
Matthieu Raynal, ${ }^{a, b}$ Pablo Ballester, ${ }^{a, c}$ Anton Vidal-Ferran ${ }^{a, c}$ and Piet W. N. M. van Leeuwen ${ }^{a}$
}

Supramolecular catalysis is a rapidly expanding discipline which has benefited from the development of both homogeneous catalysis and supramolecular chemistry. The properties of classical metal and organic catalysts can now be carefully tailored by means of several suitable approaches and the choice of reversible interactions such as hydrogen bonds, metal-ligand, electrostatic and hydrophobic interactions.

10 The first part of these two subsequent reviews will be dedicated to catalytic systems for which noncovalent interactions between the partners of the reaction have been designed although mimicking enzyme properties has not been intended. Ligand, metal, organocatalyst, substrate, additive, and metal counterion are reaction partners that can be held together by non-covalent interactions. The resulting catalysts possess unique properties compared to analogues lacking the assembling properties. Depending

15 on the nature of the reaction partners involved in the interactions, distinct applications have been accomplished, mainly: i) the building of bidentate ligand libraries (intra ligand - ligand), ii) the building of di- or oligonuclear complexes (inter ligand - ligand), iii) the alteration of the coordination spheres of a metal catalyst (ligand - ligand additive), and iv) the control of the substrate reactivity (catalyst -

substrate). More complex systems that involve the cooperative action of three reaction partners have also

20 been disclosed. In this review, special attention will be given to supramolecular catalysts for which the observed catalytic activity and/or selectivity have been imputed to non-covalent interaction between the reaction partners. Additional features of these catalysts are the easy modulation of the catalyst performance by modifying one of its building blocks and the development of new catalytic pathways/reactions not achievable with classical covalent catalysts.

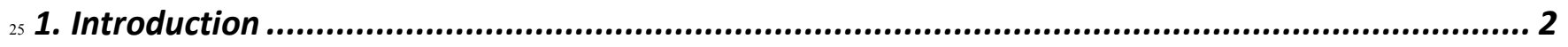

2. Concepts for the design of supramolecular catalysts beyond enzyme mimics............................ 4

3. Ligand - ligand interaction ............................................................................ 5

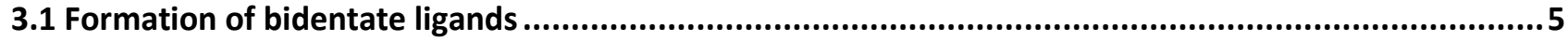

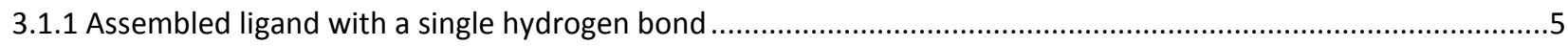

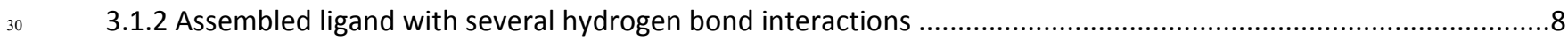

3.1.3 Lewis acid - Lewis base and ionic metal-ligand interactions ......................................................................13

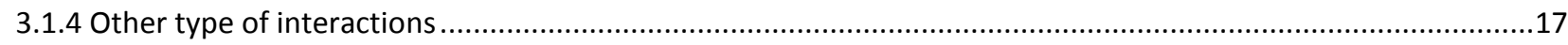

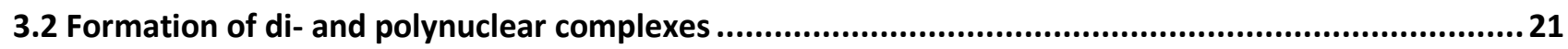

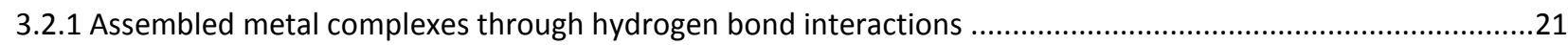

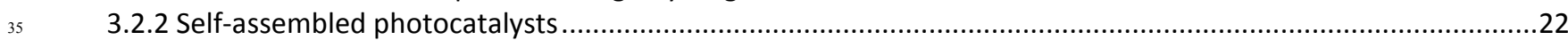

4. Ligand - ligand additive interaction.................................................................... 24

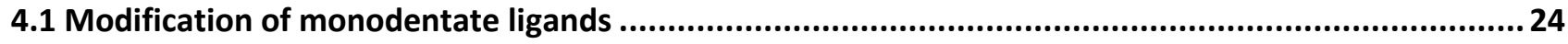

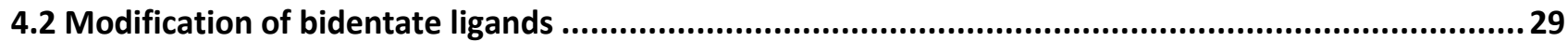

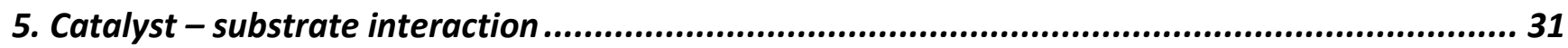




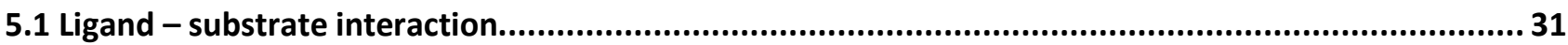

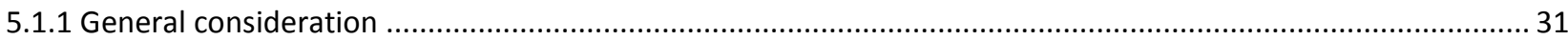

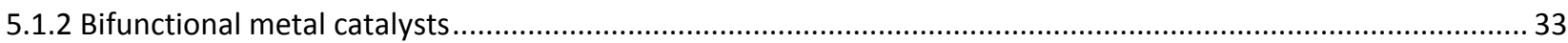

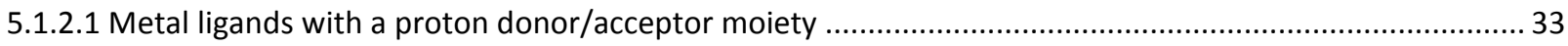

5.1.2.2 Metal ligands with tethered ionic or hydrogen bond donor/acceptor groups ..................................... 39

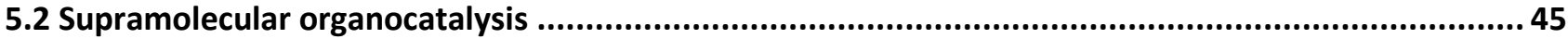

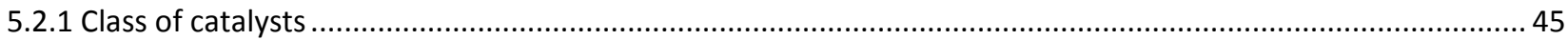

5.2.2 Modularly designed supramolecular organocatalysts ........................................................................47

5.2.2.1 Self-assembly of two monofunctional catalysts ...............................................................................4 47

5.2.2.2 MDOs composed of an organocatalyst and one or several additives...............................................48

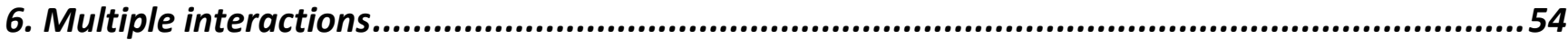

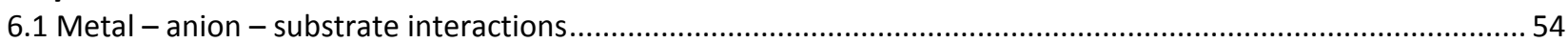

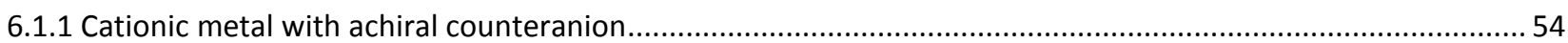

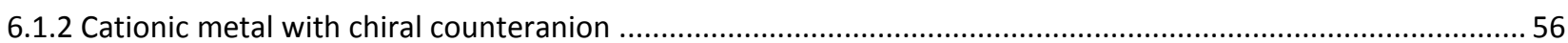

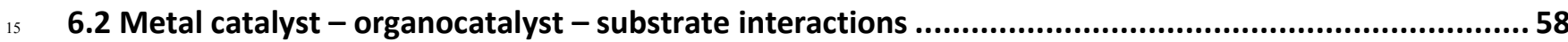

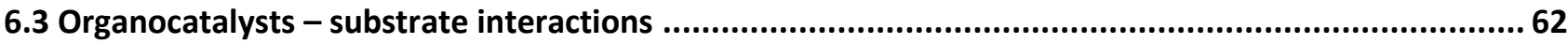

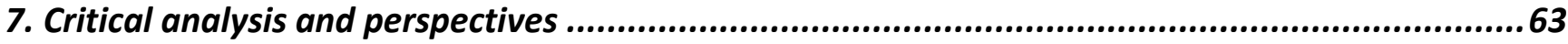

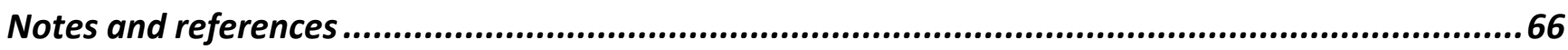

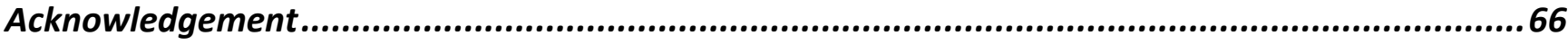

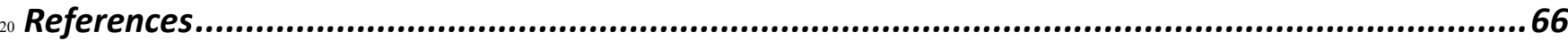

\section{Introduction}

In the last two decades supramolecular catalysis has undergone an exponential growth after a long period of a steady growth. One 25 might say that in general the attention devoted to both supramolecular phenomena and catalytic processes has increased enormously within the area of chemistry. Supramolecular chemistry nowadays plays an important role in many developments such as for example materials technology, medicine, 30 data storage, sensor technology, conversion of light to energy, selective complexation for extraction, and catalysis. Catalysis is also a keystone in chemical sciences supported by the improvement of known industrial reactions, the development of new reaction pathways and the exponential burgeoning of 35 organocatalysis. In part the growth of supramolecular catalysis should be ascribed to a refocusing or renaming of research activities, as also happened in the 1980s to supramolecular chemistry, which from then on embraces selective complexation of metal ions and anions, host-guest chemistry, crown ether 40 chemistry, self-assembly phenomena, encapsulation processes, self-recognition phenomena, etc. Also activities in the area of coordination chemistry are encountered now under the keyword supramolecular, and as we will see from the many examples in the present review this makes sense. Catalysis by itself could be ${ }_{45}$ described as a supramolecular phenomenon, as the catalyst "recognizes" substrates, "organises" the substrates in a certain way, and "assembles" a new molecule. To avoid this confusion, the name supramolecular catalysis is reserved for those reactions that involve supramolecular interactions that do not form part of
50 the basic catalytic reaction. There may not always be a clear-cut division: in the case of reactions embracing ligand - substrate interactions, one $\mathrm{N}-\mathrm{H}$ proton of the ligand interacts with the carbonyl oxygen of the substrate through a hydrogen bond interaction and subsequently reacts with it (the so-called $\mathrm{N}-\mathrm{H}$ 55 effect). ${ }^{1-3}$ Additionally, the building of supramolecular catalysts must incorporate an important degree of design. Weak interactions (cation- $\pi, \mathrm{C}-\mathrm{H}-\pi, \pi-\pi$ ) and steric repulsion play a central role in the catalyst coordination sphere by controlling the reaction paths, e.g. favouring/disfavouring the formation of a single isomer in 60 enantioselective catalysis., ${ }^{4,5}$ However, these non-covalent intermolecular forces are hardly predictable and so far cannot be used by a chemist who wishes to design "a priori" a catalyst with assembling properties.

This first review discusses how supramolecular interactions ${ }_{65}$ between complementary groups can be used as a tool to assemble the various components of the "catalytic pool" (i.e. the ligands, the metal, the organocatalyst, the substrate, the ligand additive and the metal counterion). The resulting supramolecular catalytic system is composed of assembled building blocks having improved 70 properties compared to the non-assembled catalyst. The driving idea is to modulate the properties of a given catalyst in a facile fashion; it avoids the modification of the catalyst backbone by means of painstaking synthesis as usually required to improve the catalytic properties (rate, selectivity, and substrate scope) of 75 covalent catalysts. Among other applications, it allows for the construction of supramolecular bidentate ligand (intra ligand ligand interaction) or polynuclear catalyst (inter ligand - ligand) libraries, for the tuning of the coordination spheres of a metal catalyst (ligand - ligand additive) and for the control of the 


\section{Cite this: DOI: $10.1039 / \mathrm{c0xx00000x}$}

ARTICLE TYPE

reactivity of a substrate (catalyst - substrate). Even though substrate preorganisation and transition state stabilisation are possible modes of action of any of these catalytic systems, the strategies presented here differ from the building of enzyme ligand-ligand interaction (section 3)
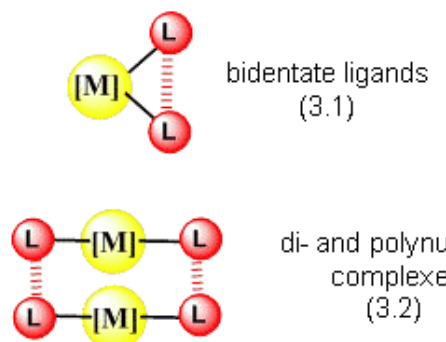

di- and polynuclear complexes

(3.2)

\section{ligand-ligand additive interaction (section 4)}

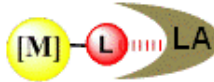

modification of monodentate ligands (4.1)

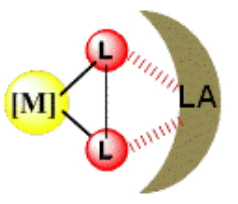

modification of bidentate ligands (4.2)

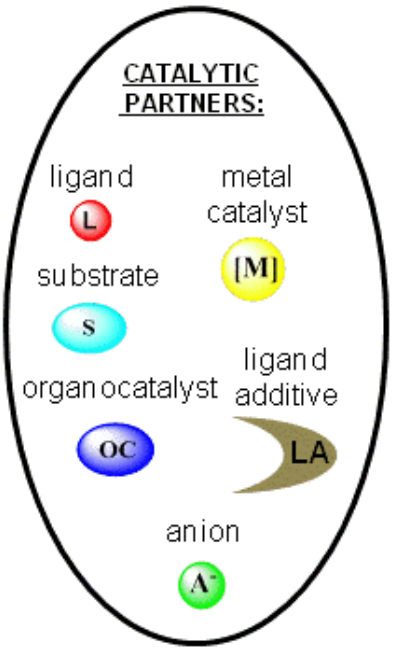
catalyst - substrate interaction (section 5)

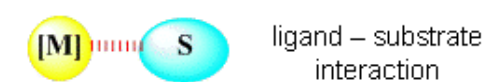

(5.1)
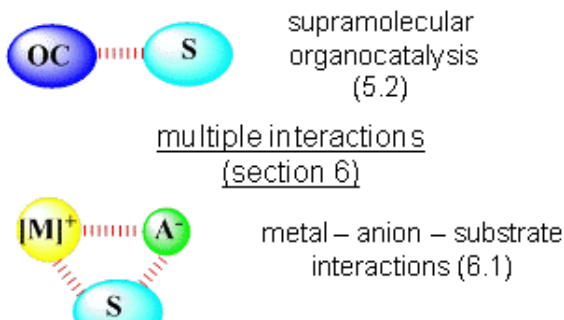

S

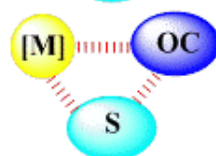

metal catalyst organocatalyst - substrate interaction (6.2)

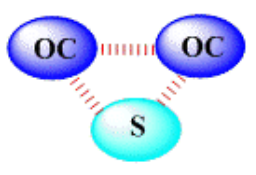

organocatalysts substrate interaction (6.3)

Scheme 1 Supramolecular catalysts beyond enzyme mimics. The dashed red lines represent the non-covalent interaction. In the text, formulas will be written as $\mathbf{L}-\mathbf{L}$ (ligand - ligand interaction) and $\mathbf{L} \cdot \mathbf{L A}$ (ligand - ligand additive interaction).

mimics because none of the properties of enzymes is specifically targeted. Part 2 of this review will be dedicated to enzyme 10 mimics $^{6-11}$ including catalysis in confined environments, ${ }^{12-16}$ catalysis in water, ${ }^{17,18}$ self-replicators, ${ }^{19-27}$ allosteric catalysts, ${ }^{28-30}$ and biomacromolecule hybrids as metal and organic catalysts. ${ }^{31-35}$ The non-covalent immobilization of homogeneous catalysts on soluble or solid support, ${ }^{3,36-39}$ self-supported catalysts, ${ }^{40}$ 15 heterogeneous catalysts modified with chiral additives, ${ }^{41,42}$ and related systems ${ }^{43}$ are out of the scope of this review.

Previous reviews ${ }^{5,39,44-53}$ and a book ${ }^{54}$ on the discipline of supramolecular catalysis beyond enzyme mimics deal with a limited number of topics or only to the authors' own contributions.

${ }_{20}$ Given the rapid growth of the discipline, many innovative catalytic systems and strategies have been achieved since the last reviews. The originality of this review is summarized in the three following points. First, supramolecular catalysts will be classified according to the design strategy outlined by the authors. Again, the borders

25 between these strategies are flexible since e.g. a ligand can interact both with another ligand and the substrate during the various steps of the catalytic cycle. Well-chosen control experiments and suitable analyses are required to precisely determine the nature of the non-covalent interactions and the reaction partners involved in 30 the rate and selectivity-determining steps. We will particularly point out examples for which the interaction at the origin of the better activity/selectivity is different from the one initially planned by the authors. Second, supramolecular catalysts can self-assemble in situ by mixing different building blocks; this strategy presents 35 advantages compared to the ones that require isolation of the precatalysts prior to catalysis. It also allows for a fine tuning of the performance of the catalyst by modifying one of its building blocks. Examples of the construction of metal catalyst libraries will be provided; each catalyst differs by its electronic and steric 40 properties as well as its chiral environment. Finally, examples of organic catalysts will be mentioned even though organocatalysts are not usually considered to belong to the supramolecular catalysis discipline because reversible interactions between the catalyst and the substrate(s) or reaction intermediates are 45 ubiquitous in the catalytic cycle. However, some of the strategies mentioned above for metal catalysts (e.g. the construction of catalyst libraries and the modulation of the electronic/steric properties and the chiral environment of the catalyst) have been applied with success to classical organocatalysts. Accordingly, it 50 appeared to us that the combination of supramolecular metal catalysts and supramolecular organocatalysts in the same review can stimulate the development of innovative catalysts and open new avenues in both directions.

This review is organised according to the nature of the reaction ${ }_{55}$ partners that interact during the catalytic process. This seemed more logical than a classification based on the type of non-covalent interactions involved, since the same interaction can be used for 
many different applications. After a short description of the concepts inherent to supramolecular catalysts (section 2), we will provide a detailed overview of existing examples of catalytic reactions relying on the interactions between two ligands (section 53 ), between one ligand and one ligand additive (section 4) and between one metal or one organic catalyst and a substrate (section 5). In a few cases, more than two reaction partners are involved in the self-assembled catalysts; some of these examples will be disclosed in section 6. In the last section, we will present our 10 critical analysis of various strategies used in the construction of supramolecular catalysts that go beyond enzyme mimics.

\section{Concepts for the design of supramolecular catalysts beyond enzyme mimics}

A metal catalyst (comprising the ligand(s), the counterion and the 15 metal) or an organocatalyst and the substrate(s) are the usual partners involved in a catalytic process. The control of the process in terms of reactivity and selectivity depends on the mutual interactions established between the partners along the whole catalytic cycle. Non-covalent intermolecular forces, mainly 20 hydrogen bonding, electrostatic, Lewis acid - Lewis base and hydrophobic, are reversible interactions that can allow for a fine tuning of the properties of a given catalytic system (Scheme 1). ${ }^{55}$

Until very recently, the building of a metal catalyst only referred to the coordination of one or several ligands with purely 25 covalent structure with the metal centre. Nowadays, supramolecular interactions have been used to self-assemble ligands and to tune the coordination spheres of metal catalysts. The first topic encompasses the building of libraries of supramolecular bidentate ligands via intramolecular interactions established 30 between monodentate ligands. Since the initial work of Breit et $a l .{ }^{56}$ involving hydrogen-bonded monophosphines, the diversity of the interactions used to assemble the ligands and the catalytic applications of these supramolecular bidentate ligand libraries have increased exponentially (section 3.1). Additionally, 35 intermolecular interactions between ligands have also been used in the construction of hydrogen-bonded metal complexes or selfassembled photocatalysts (section 3.2). The first coordination sphere consists of the solvent molecules and the part of the ligands that are connected directly to the metal atom. The metal 40 counterions, the molecules that interact with the ligands through non-covalent interactions and remote portions of the ligand backbone define the second coordination sphere. Classical catalytic systems are built on a covalent skeleton that can only be modified by means of painstaking synthesis. In 2001, the group of ${ }_{45}$ Reek and van Leeuwen ${ }^{57}$ used Lewis acid - Lewis base interactions (coordination bonds) to assemble a pyridylphosphine ligand and a $\mathrm{Zn}$-porphyrin unit (the ligand - template strategy). The first and second coordination spheres of the self-assembled catalyst can be modulated in a facile fashion by changing the nature 50 of either the pyridylphosphine ligand or the Zn-porphyrin unit coordinated to the periphery of the catalytic metal centre. From that time, the use of additives able to establish reversible interactions with monodentate and bidentate ligands proved to be useful in fine tuning the electronic, steric and chiral properties that the ligands 55 provide to the metal (section 4). Of special interest to supramolecular catalysts based on ligand - ligand or ligand additive interaction is the fact that the properties of the self- assembled catalyst can be modulated by simple modification of one of its components: ligands or additives. Accordingly, we will 60 present several examples of self-assembled metal catalysts prepared in situ by simply mixing different building blocks.

A good understanding of the nature of the interaction that takes place between the substrate(s) and the catalyst is key to optimise the activity and the selectivity of a given catalytic process. In 65 transition-metal catalysis, this fact has shown to be highly relevant since the discovery of $\mathrm{Ru}$ (diamine) hydrogenation catalysts by Noyori and co-workers in $1995 .^{58-60}$ This class of $\mathrm{Ru}$ catalysts constitute a seminal example of metal bifunctional catalysts in which the hydride and the amine ligand act cooperatively in 70 activating the carbonyl substrate. ${ }^{1-3}$ Ligand - substrate interactions have been incorporated in the design of several catalysts (section 5.1). A plethora of organocatalysts operate via the formation of non-covalent interactions between the catalyst and the substrate(s), the reaction intermediate(s) or the transition state., ${ }^{4,5,61-70}$ Of 75 interest to this review is the design of organocatalysts using approaches similar to those used for self-assembled metal catalysts (section 5.2). Modularly designed organocatalysts (MDOs) are composed of several building blocks connected to one another by means of non-covalent interactions between the complementary 80 groups hold by the blocks. Accordingly, libraries of self-assembled organocatalysts can be achieved by a careful choice of the building blocks, in a similar fashion as self-assembled metal catalysts mentioned above.

In a few cases, non-covalent interactions involving more than 85 two reaction partners have been detected in the selectivity-determining step of the catalytic reaction. Metal counteranions play a critical role in catalysis, ${ }^{53,71}$ and in some cases the interactions of the substrate with both the metal counteranion and the ligand are the origin of the observed selectivity (section $9_{90} 6.1$ ). Well-defined transition states that involve three reaction partners have been demonstrated for a few catalytic reactions: the substrate interacts with a metal catalyst and an organocatalyst (section 6.2) or with two organocatalysts (section 6.3). ${ }^{72}$ In these cases, the substrate is activated simultaneously by two catalysts 95 through several types of non-covalent interactions.

Many supramolecular strategies that have recently emerged for promoting chemical reactions go beyond the traditional approaches of homogeneous catalysis. A certain degree of catalyst design can be achieved by incorporating recognition units to it. A 100 specific application will be targeted in each strategy depending on the nature of the interacting partners. However, we will show that borders between the different approaches are not clear-cut and interactions that differ from those designed initially can merge. For example, a urea motif may not only play the role of recognition 105 unit in the construction of a bidentate ligand, it can also be involved in mediating the reactivity of a nucleophile through hydrogen bond interactions. ${ }^{73}$ Additionally, the design strategy outlined by the authors may be based on the use of one or two main non-covalent interactions, but interactions not foreseen in the initial design of 110 the selective catalyst can play an important role in the catalytic process. ${ }^{4,5}$ 


\section{Cite this: DOI: 10.1039/c0xx00000x}

ARTICLE TYPE

\section{Ligand - ligand interaction}

\subsection{Formation of bidentate ligands}

The "natural bite angle" is an intrinsic property of bidentate ligands which strongly influences the course of a given catalytic reaction.

5 The design of wide bite angle diphosphines led to a major breakthrough in the domain of palladium-catalysed cross-coupling reactions and rhodium-catalysed hydroformylation, among other catalytic applications. ${ }^{74-77}$ In this field, subtle variations of the diphosphine's bite angle and of the ligand's geometry produce 10 drastic effects in catalysis. The assembly of supramolecular bidentate ligands based on two monodentate ligands held together by means of hydrogen bonds, Lewis acid -Lewis base interactions and ionic metal-ligand interactions, was the subject of intense research during the last ten years. The use of such supramolecular 15 ligands presents many advantages: i) it reduces the amount of synthetic work required for the preparation of a similar range of covalent ligands, ii) by preparing two groups of $m$ and $n$ monodentate ligands, which combine as a 1:1 complex, an $m \times n$ library of ligands is obtained, iii) if $m$ and $n$ possess 20 complementary building blocks, the heterocombination of ligands will be favoured. Such ligand libraries are important since they enable catalyst optimisation using combinatorial/deconvolution approaches but can also allow for the discovery of new catalytic reactions or privileged ligands able to catalyse selectively different 25 reactions with diverse modes of reactivity. Supramolecular bidentate ligands can thus fill the gap between purely combinatorial strategies and an approach involving the covalent synthesis of modular ligands. $^{78}$

The research on supramolecular bidentate ligands has increased 30 enormously since the report of Breit et al. on hydrogen-bonded monophosphines based on the 2-hydroxypyridine/2-pyridone recognition motif. ${ }^{56}$ Most of the existing examples will be described in the following section with a special focus on efficient catalytic systems that can be easily modulated. ${ }^{39,44,45,47,49-51,78,79}$

\section{3.1.1 Assembled ligand with a single hydrogen bond}

Two monodentate ligands can be held together in a 1:1 complex by means of very strong interactions (electrostatic interactions can be as strong as covalent bonds and ionic hydrogen bonds can be maintained in water). In the early avant la lettre examples, 40 hydrogen-bonded bidentate ligands were neither designed nor envisaged as supramolecular ligands. This is the case for the SPO (secondary phosphine oxide) ligands which have been known for 45 years as ligands for transition-metals and introduced as catalysts by van Leeuwen and Roobeek in the early $1980 \mathrm{~s} .{ }^{80}$ The pentavalent ${ }_{45}$ phosphine oxide is in equilibrium with the trivalent phosphinous acid (Fig. 1, a). Upon coordination to a soft transition-metal, the cis-coordinated SPO ligands usually interact by means of a hydrogen bond established between the $\mathrm{O}-\mathrm{H}$ proton of a neutral SPO ligand and the oxygen atom of an anionic SPO ligand. 50 Accordingly, the SPO ligands act as a monoanionic diphosphorus chelate for the metal centre. For
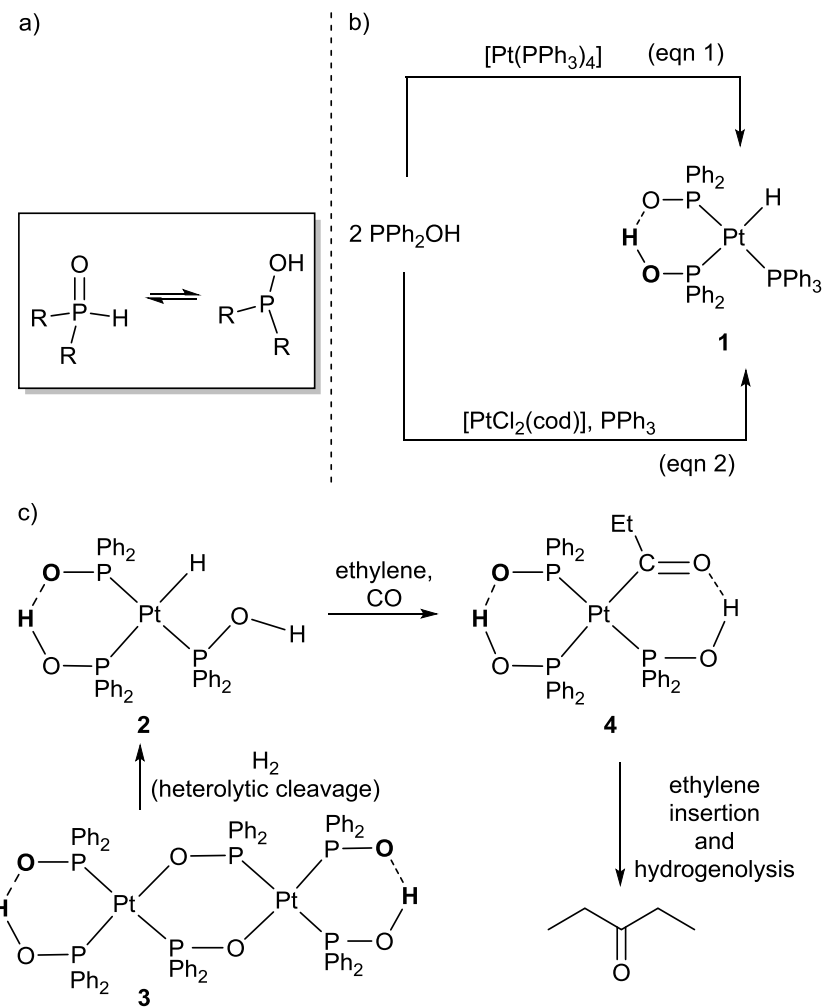

Fig. 1 SPO: an early example of supramolecular bidentate ligand. a) Tautomeric equilibrium between pentavalent and trivalent SPO ligand. b) 55 Preparation of $\mathbf{1}$, an hydroformylation and hydrogenation catalyst. c)

Formulas of monomer $\mathbf{2}$ or dimer $\mathbf{3}$ and plausible mechanism for the formation of pentan-3-one from 4 observed during ethylene hydroformylation. Refs: see the text.

example, two equivalents of $\mathrm{PPh}_{2} \mathrm{OH}$ react with $\left[\mathrm{Pt}\left(\mathrm{PPh}_{3}\right)_{4}\right]$ to 60 yield $\left[\mathrm{Pt}(\mathrm{H})\left(\mathrm{PPh}_{2} \mathrm{O}-\mathrm{PPh}_{2} \mathrm{OH}\right)\left(\mathrm{PPh}_{3}\right)\right] \mathbf{1}$ (eqn 1, Fig. 1, b). ${ }^{81}$ The same complex can be generated by mixing $\left[\mathrm{PtCl}_{2}(\operatorname{cod})\right], \mathrm{PPh}_{2} \mathrm{OH}$ and $\mathrm{PPh}_{3}$ (eqn 2, Fig. 1, b). ${ }^{80,82}$ The nature of the added phosphine was varied and the resulting complexes proved to catalyse the hydroformylation of alkenes, and the hydrogenation of alkenes and ${ }_{65}$ aldehydes. Stoichiometric reaction led to the identification of each intermediate in the hydroformylation reaction of ethene (Pt-propyl, Pt-propionyl). Most likely, the rate determining step is the hydrogenolysis of the platinum acyl bond. Trialkylphosphine oxides $\mathrm{R}_{3} \mathrm{P}=\mathrm{O}$ molecules are known to be one of the best hydrogen 70 bond $^{\text {acceptors }}{ }^{83}$ and apparently SPO ligands have similar properties. The use of SPO ligands was recently recognized as the first use of a supramolecular bidentate ligand in catalysis. ${ }^{84}$ When $\mathrm{PPh}_{3}$ in eqn 2 is replaced by $\mathrm{PPh}_{2} \mathrm{OH}$ a complex is formed which contains two SPO ligands in a monoanionic bidentate fashion and 75 one monoligated $\mathrm{PPh}_{2} \mathrm{OH}$ per platinum. The structure of this complex was not established due to its low solubility; it could be a monomeric complex $\mathbf{2}$ or a dimer (such as $\mathbf{3}$ ) or even an oligomeric complex (Fig. 1, c). Stoichiometric reaction of this complex with ethene and $\mathrm{CO}$ led to the unambiguously identified complex 4 80 where the two trans 


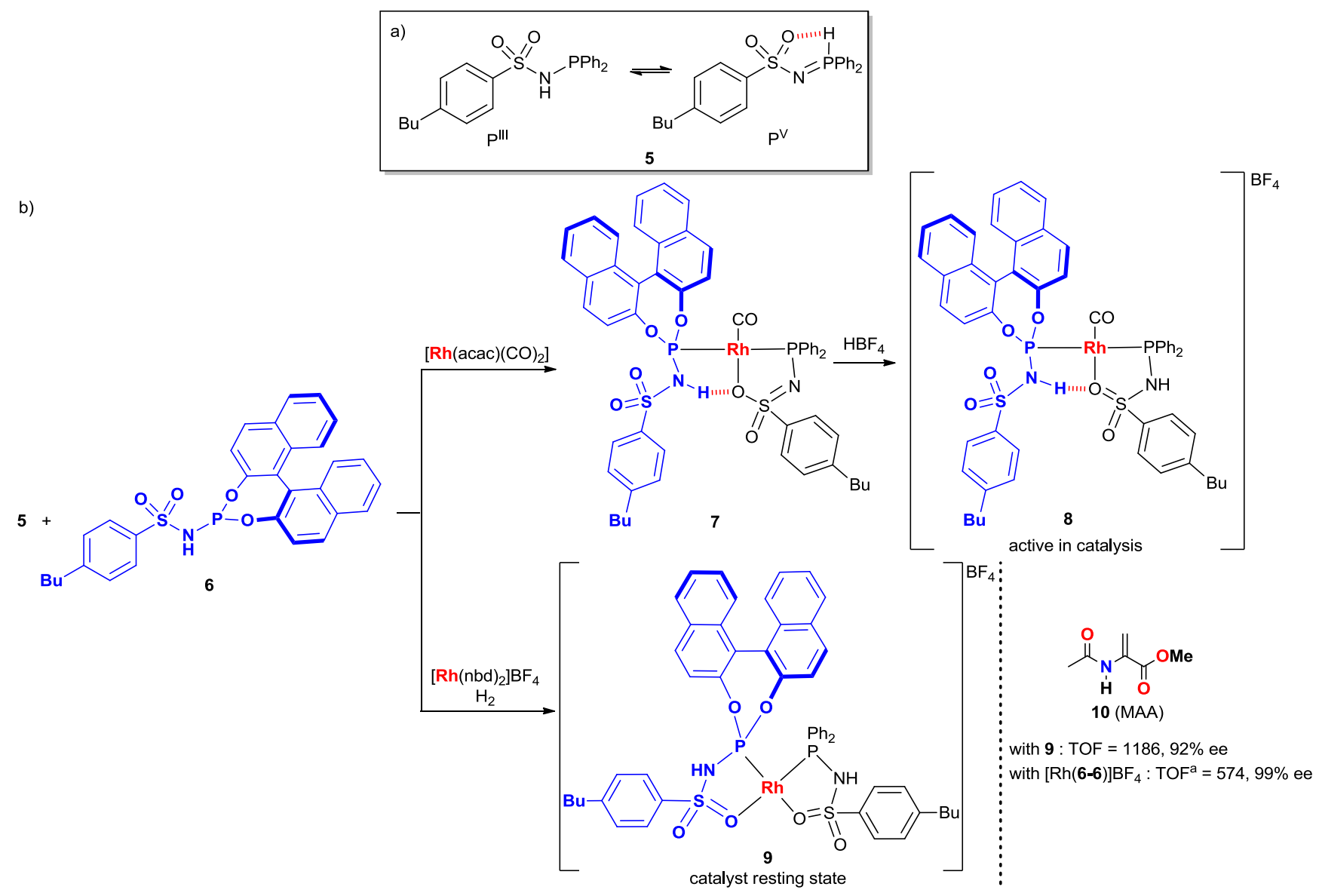

Fig. 2 METAMORPhos ligands. a) Tautomeric equilibrium between pentavalent and trivalent ligand $\mathbf{5}$. b) In the hetero-complexes $\mathbf{7}$ and $\mathbf{8}$, ligands $\mathbf{5}$ and $\mathbf{6}$ are assembled via a single hydrogen bond. In the asymmetric hydrogenation of 10, the hetero-complex 9 bearing a ligand heterocombination of 5 and $\mathbf{6}$ is more active than the homo-complex of ligand $\mathbf{6}$. TOF in mol.mol ${ }^{-1} \cdot \mathrm{h}^{-1}$. MAA $=$ methyl 2-acetamidoacrylate. Ref: see the text.

SPO ligands are bound in an alternating manner through a hydrogen bond to the acyl and phosphine oxide groups, respectively. Catalyst 2 behaves quite differently from 1 since pentan-3-one is observed during ethene hydroformylation 10 experiments. Pentan-3-one is formed by ethene insertion into the $\mathrm{Pt}-\mathrm{C}$ bond of the Pt-propionyl intermediate, confirming that hydrogenolysis is the slow step in this class of catalysts. ${ }^{82}$ Recent evaluation of SPO and HASPO (HeteroAtom-substituted SPO) ligands in the rhodium-catalysed hydroformylation reaction of 15 alkenes highlights their non-innocent nature; nucleophilic addition of the $\mathrm{P}-\mathrm{H}$ bond to aldehydes yields $\alpha$-hydroxyphosphane oxides. ${ }^{85,86}$ Owing to the excess of ligands usually used in hydroformylation, this side reaction reduces the amount of available SPO ligands. When introduced, rates and selectivities of ${ }_{20} \mathrm{Pt}-\mathrm{SPO}$ catalysts were of interest, but today they have lost importance.

Recently, SPOs and HASPOs emerged as a very efficient class of ligands in palladium-catalysed cross-coupling reactions, involving challenging aryl-chloride and even aromatic $\mathrm{C}-\mathrm{H}$ bonds 25 as electrophiles, and in palladium-catalysed asymmetric allylic substitution reactions amongst other reactions. ${ }^{84,87-89} \mathrm{~A}$ recent study highlights the versatile coordination mode of SPO ligands: monoligated neutral, monoligated anionic, bis-ligated with a bridging hydrogen bond and bis-ligated with a bridging metal. ${ }^{90}$

${ }_{30}$ One may wonder whether the bidentate mode of SPO ligands is maintained during the catalytic experiment, mainly because the bridging proton is acidic. However, the superiority of SPOs over non-assembled phosphine ligands in several catalytic reactions, working under mild conditions, such as alkene 35 hydroformylation, ${ }^{80,82,85,86}$ alkene and imine hydrogenation ${ }^{91-93}$ and nitrile hydration ${ }^{94-96}$ argues in favour of the bidentate coordination mode of SPOs maintained during the catalytic process. Castro et al. showed that a rhodium complex containing BINOL-derived chiral SPO ligands catalysed the asymmetric 40 transfer hydrogenation of acetophenone with $89 \%$ enantiomeric excess (ee), employing isopropanol as the hydrogen-donor. ${ }^{97}$ 


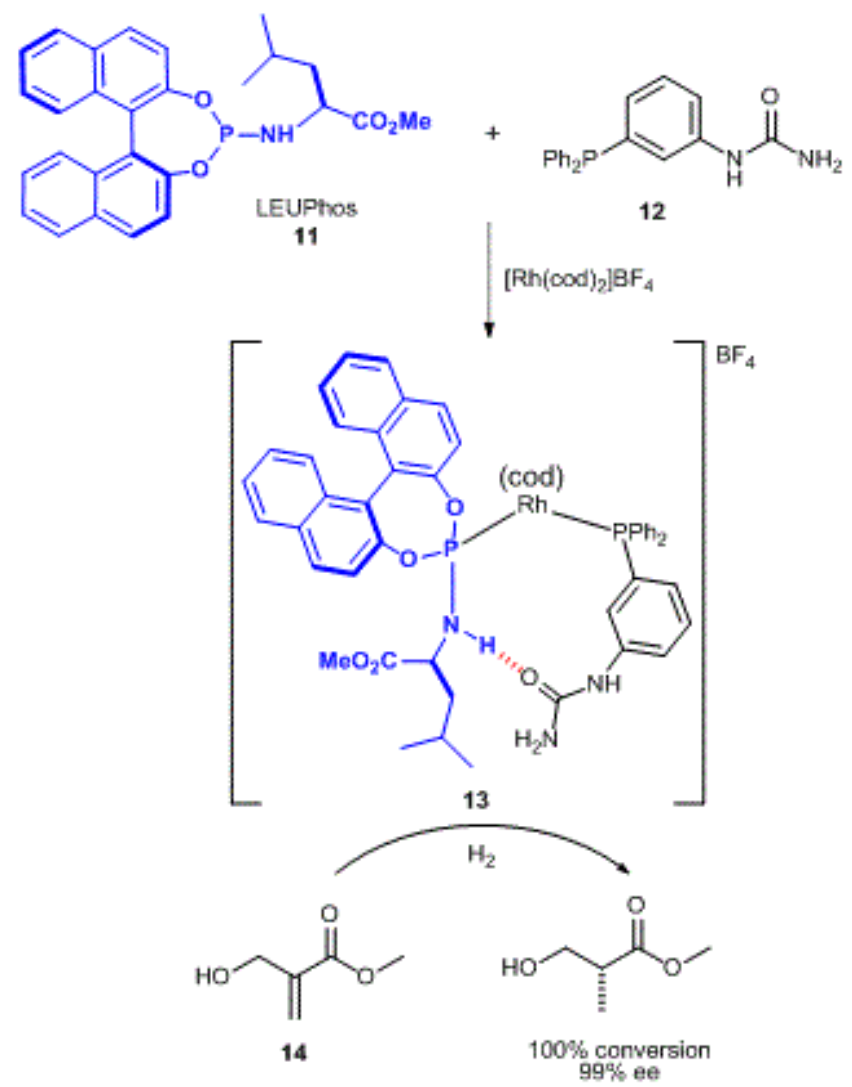

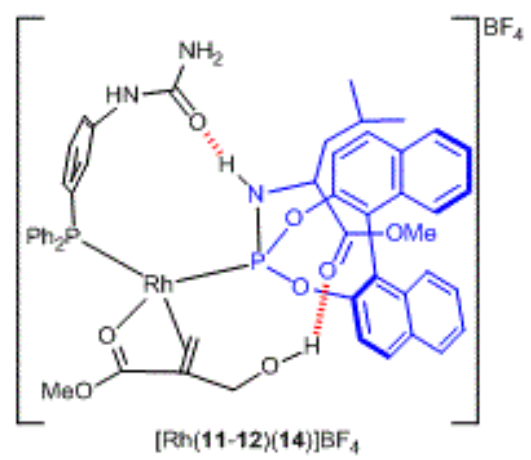

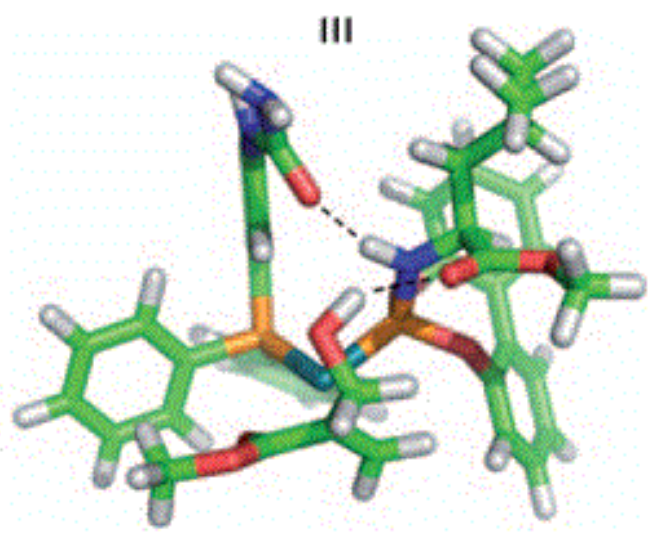

Fig. 3 LEUPhos-UREAPhos supramolecular ligand assembled via a single hydrogen bond. Left: Catalyst derived from 13 provided the hydrogenation product of 14 with $99 \%$ ee. Right: Energy-minimized structure of [Rh(11-12)(14)]BF, BINOL backbone omitted for clarity. (The energy-minimized structure is reprinted with permission from ref. 103. Copyright 2009. John Wiley and Sons).

5 Mechanistic investigation reveals that the dehydrogenation of isopropanol is assisted by one of the oxygen atom of the chelating SPO ligands. The resulting $\mathrm{O}-\mathrm{H}$ proton of the SPO ligand and the rhodium hydride are likely transferred to the carbonyl group of acetophenone via a well-defined seven-membered transition state.

${ }_{10}$ A similar outer-sphere mechanism has been proposed for the Shvo catalyst. ${ }^{98,99}$ Other examples of metal bifunctional complexes that mediate the selectivity of a catalytic reaction by means of ligand substrate interactions will be mentioned in section 5.1.2.

Patureau et al. developed a new class of ligands, sulfonamido15 phosphinamides, which are subject to a $\mathrm{P}^{\mathrm{III}} / \mathrm{P}^{\mathrm{V}}$ tautomerism similar to that observed in SPOs (Fig. 2, a). ${ }^{100}$ Upon coordination of two different sulfonamido-phosphinamide ligands (one achiral $\mathbf{5}$ and one chiral 6) to $\left[\mathrm{Rh}(\mathrm{acac})(\mathrm{CO})_{2}\right]$, only the hetero-complex 7 is formed, i.e. the complex which contains a heterocombination of 20 ligands $\mathbf{5}$ and $\mathbf{6}$. The structure of $\mathbf{7}$ presents some additional remarkable features: i) within this complex, ligand $\mathbf{5}$ is deprotonated and acts as a $(P, O)$-chelate, ii) the phosphorus atoms of $\mathbf{5}$ and $\mathbf{6}$ are mutually trans and, iii) ligands $\mathbf{5}$ and $\mathbf{6}$ are assembled by means of a hydrogen bond established between the $\mathrm{N}-\mathrm{H}$ proton ${ }_{25}$ of $\mathbf{6}$ and the rhodium-coordinated oxygen atom of $\mathbf{5}$. Accordingly, the resulting assembled ligand 5-6 defines a supramolecular anionic $\kappa^{3}-P, O, P$ pincer ligand. The cationic rhodium complex obtained upon protonation of 7 with $\mathrm{HBF}_{4}$ is active in catalysis (Fig. 2, b). When $\left[\mathrm{Rh}(\mathrm{nbd})_{2}\right] \mathrm{BF}_{4}$ is used as the precursor, the 30 catalyst resting state for the hydrogenation reaction is also a heterocomplex (9 Fig. 2, b) but this time ligands 5 and $\mathbf{6}$ both act as neutral $(P, O)$-chelates for the rhodium metal centre and no hydrogen bond is observed between 5 and 6. Complex 9 hydrogenates substrate $\mathbf{1 0}$ with good activity $(\mathrm{TOF}=1186$ $\left.35 \mathrm{~mol} \cdot \mathrm{mol}^{-1} \cdot \mathrm{h}^{-1}\right)$ and enantioselectivity ( $92 \%$ ee). The catalyst which contains only the chiral ligand 6 is slightly more selective ( $99 \%$ ee) but twice less active than 9. This class of ligands is named METAMORPhos owing to their adaptability and dual character. Pchirogenic METAMORPhos ligands were prepared but they are ${ }_{40}$ poor enantioselective catalysts. ${ }^{101}$ A METAMORPhos and UREAPhos (see below) mixed ligands library was screened for the asymmetric hydrogenation of a challenging cyclic imine. ${ }^{102}$ The best combination gave $95 \%$ ee ( $61 \%$ conversion). However, the origin of the superiority of heterocombinations incorporating 45 METAMORPhos ligands 
(a)

2

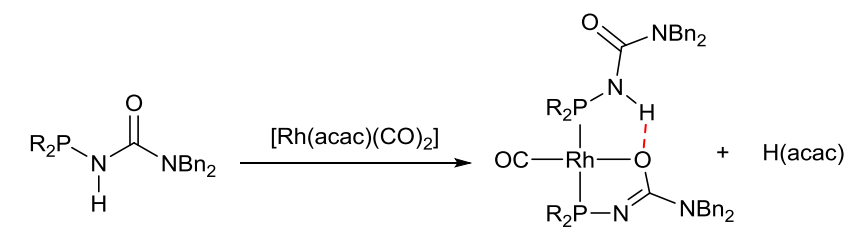

(b)

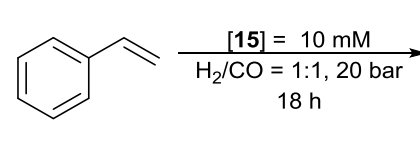

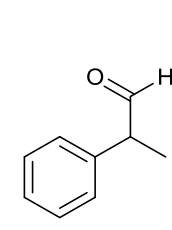

$75 \%$ conversion b:I ratio $=27$ $46 \%$ ee

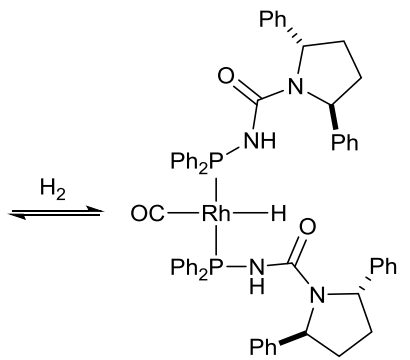

Fig. 4 Assembled UREAPhos ligands via a bridging hydrogen atom. (a) Complex formed by reacting $N, N$-dibenzyl UREAPhos ligand with $\left[\mathrm{Rh}(\mathrm{acac})(\mathrm{CO})_{2}\right]$ (b) Complex 15 is an active catalyst for the hydroformylation of styrene, giving the branched product as the main isomer albeit with moderate ee. Refs: see the text.

5 remains to be clarified. Kinetic experiments indicated that the mechanism of the hydrogenation reaction differs depending on the nature of the ligands present in the catalyst resting state. For $\mathbf{9}$, it was suggested that an unusual oxidative addition of the $\mathrm{N}-\mathrm{H}$ bond of the METAMORPhos ligand occurs in the catalytic cycle, 10 yielding a Rh hydride species that can split the hydrogen molecule heterolytically. In light of the proposed catalytic cycle, it seems that the efficiency of METAMORPhos ligands is more due to their electronic properties and their ability to activate the substrate $\left(\mathrm{H}_{2}\right)$ than to ligand - ligand interactions.

15 Breuil et al. reported that LEUPhos 11, a chiral phosphoramidite ligand derived from leucine, and an achiral UREAPhos ligand 12 self-assembled upon coordination to a $[\mathrm{Rh}(\mathrm{cod})]^{+}$fragment. ${ }^{103}$ The cis-coordinated $\mathbf{1 1}$ and $\mathbf{1 2}$ ligands act as a chelate for the rhodium metal centre as a result of a hydrogen bond established between the $20 \mathrm{~N}-\mathrm{H}$ proton of $\mathbf{1 1}$ and the carbonyl oxygen of $\mathbf{1 2}$. This attractive interaction mediates the selective formation of the hetero-complex 13 over the respective homo-complexes (Fig. 3). The catalyst derived from 13 selectively hydrogenates methyl 2hydroxymethylacrylate 14 (99\% ee). Two hydrogen bonds are 25 present in the energy-minimized structure of the catalytic intermediate $[\mathrm{Rh}(\mathbf{1 1}-\mathbf{1 2})(\mathbf{1 4})] \mathrm{BF}_{4}$ : i) one between ligands 11 and 12 and, ii) one between the hydroxyl group of the substrate 14 and the carbonyl oxygen of LEUPhos ligand 11 (Fig. 3). While the ligand - substrate interaction clearly plays a crucial role during the 30 enantio-determining step, the contribution of the ligand - ligand interaction on the catalytic efficiency has not been established. A supplementary control experiment with a $N$-methylated analogue of 11 would have given an indication.

Another example of assembled ligand through a single 35 hydrogen bond is provided by some members of the UREAPhos family. ${ }^{104,105}$ Notably, the complex formed by reacting two equivalents of a $N, N$-dibenzyl UREAPhos derivative with
$\left[\mathrm{Rh}(\mathrm{acac})(\mathrm{CO})_{2}\right]$ was characterized and presented some analogies with complex 7: i) one UREAPhos ligand acts as a $(P, O)$-chelate 40 as a result of the deprotonation of one acidic urea proton by the acac ligand, ii) the second UREAPhos is coordinated as a neutral ligand and is linked to the chelating anionic ligand via an intramolecular hydrogen bond and, iii) the rhodium complex is neutral and the phosphorus atoms are mutually trans (Fig. 4, a). A 45 similar coordination mode was observed with 1 '-(diphenylphosphino)ferrocenecarboxylic acid. ${ }^{106,107}$ During the hydroformylation reaction, the oxidative addition of dihydrogen to these precatalysts yields a catalytic resting state that contains no hydrogen bond between the UREAPhos ligands. A library of chiral ${ }_{50}$ UREAPhos ligands was investigated for the asymmetric hydroformylation of styrene with good selectivity for the branched product but modest enantioselectivity (15, Fig. 4). The mechanism involves heterolytic cleavage of $\mathrm{H}_{2}$ on the rhodium centre and an anionic phosphine urea fragment (Fig. 4, b). ${ }^{105}$

\section{3.1.2 Assembled ligand with several hydrogen bond} interactions

Since the seminal work of Breit et al. ${ }^{56}$, involving hydrogen-bonded ligands by taking advantage of the pyridone/2-hydroxypyridine tautomeric equilibrium, this area of ${ }_{60}$ research has drawn the attention of several research groups. New systems have been designed in which the monodentate ligands selfassemble by means of two, three or even more classical hydrogen bonds. In some systems, weak hydrogen bonds were also observed in the solid state and in solution as additional stabilising ${ }_{65}$ interactions. ${ }^{108}$ These hydrogen-bonded ligands behave as typical bidentate ligands in hydroformylation experiments at least upon reaction conditions that does not weaken or disrupt the assemblies. Accordingly, a significant change in the reaction outcome is usually observed when the temperature is increased or when protic 70 polar solvents are used. ${ }^{56,105,109-113}$ Several classes of ligands are now available, as libraries for catalyst optimisation. 

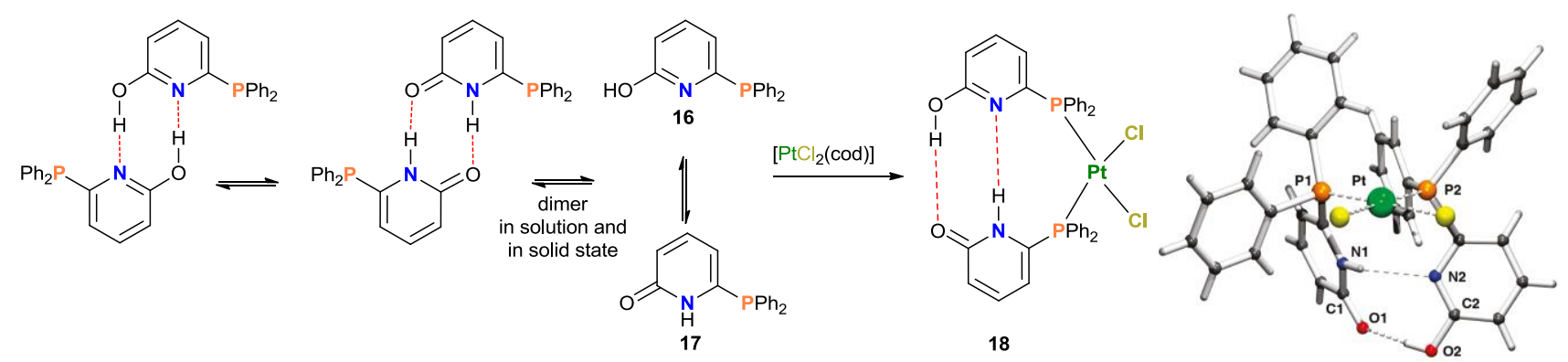

Fig. 5 Complexation properties of the pyridone/2-hydroxypyridine phosphine ligands. Tautomeric equilibrium between 16 and 17 , and coordination to Pt. Crystal structure of complex 18. (The crystal structure is reprinted with permission from ref. 118. Copyright 2011. American Chemical Society).

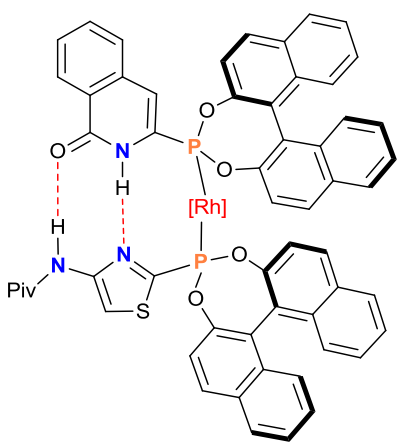

19

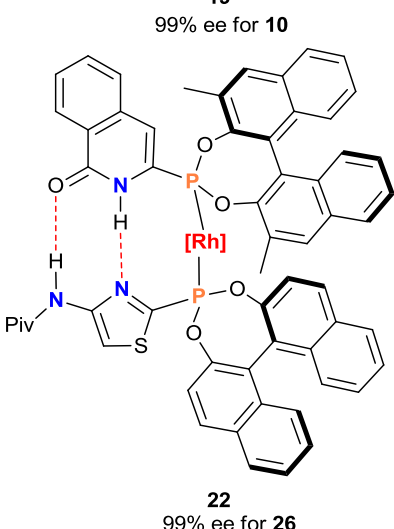

$99 \%$ ee for 26

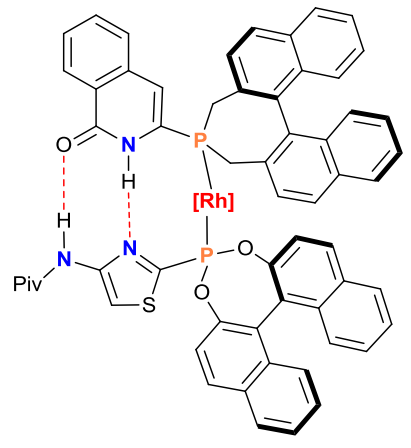

20

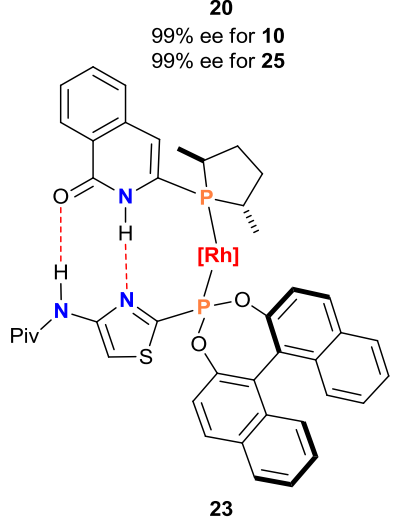

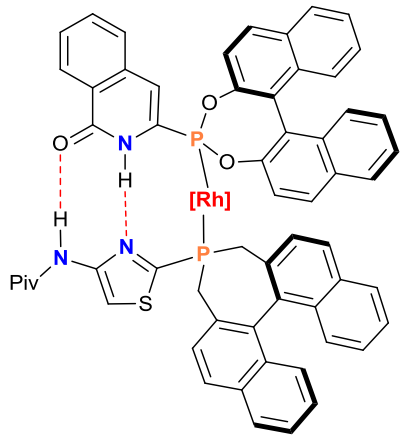

21

$99 \%$ ee for 10

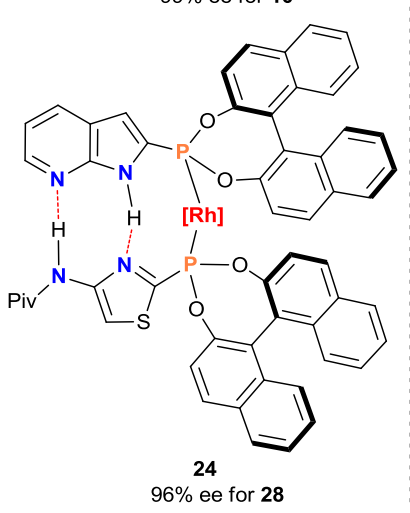

substrates:<smiles>C=C(C[NH2+])C(=O)OC</smiles>

25<smiles>COC(=O)/C(=C/c1ccccc1)NC(C)=O</smiles>

26<smiles>C=C(CC(=O)OC)C(=O)OC</smiles>

27<smiles>C=C(NC(=O)OC)c1ccccc1</smiles>

28

Fig. 6 Breit supramolecular bidentate ligands built on the adenine-thymine pair model. A combinatorial approach has been used to identify the best ligands in asymmetric hydrogenation reactions of various substrates. Phth $=$ phthaloyl. Piv = pivaloyl. Ref: see the text.

They include: pyridone/2-hydroxypyridine phosphanes, ${ }^{56,109,113-119}$ 10 amidopyridine/isoquinolone phosphanes and their derivatives built on the adenine-thymine pair model, ${ }^{110,111,120-126}$ UREAPhos and its derivatives, ${ }^{102,104,105,127-130}$ Suprabox (urea-oxazoline ligands), ${ }^{131}$ DpenPhos (based on phosphoramidite ligands), ${ }^{132}$ peptides phosphanes (included SupraPhanephos ${ }^{133}$ and ${ }_{15}$ Suprapeptiphos), ${ }^{108,112}$ and PhthalaPhos ${ }^{134,135}$ (phosphanes appended with phthalamide residue). All these examples will be succinctly described with a special attention on the more recent results $(>2009)$.

Gellrich et al. investigated in detail the tautomeric equilibrium
20 between 6-diphenylphosphanyl-2-hydroxy-pyridine (16) and 6-diphenylphosphanylpyridone (17) before and after coordination to $\mathrm{Pt}$ (complex 18, Fig. 5). ${ }^{118}$ The free ligand exists as a pyridone-pyridone dimer both in solution and in the solid state. However, upon metal coordination, one pyridone molecule 25 transforms into 2-hydroxypyridine and the favourable complementary interactions easily compensate the energy penalty (0.9 kcal.mol $\left.{ }^{-1}\right)$ associated with the tautomerization process. Spectroscopic studies showed that the two hydrogen bonds in complex 18 are involved in a fast dynamic tautomerization 30 process. For complex 18, DFT calculations led to an estimate of an 
additional enthalpic stabilisation through hydrogen bonding of 15 kcal.mol ${ }^{-1}$. Previous studies indicated that structurally-related chiral ligands, having phospholane or phosphepine groups attached to the pyridone moiety, can exhibit totally different coordination 5 behaviour depending on the metal and the co-ligands. ${ }^{114}$ IR and NMR studies gave an indication of the tautomeric form of the pyridone moiety in the precatalysts used in rhodium-catalysed hydrogenation reactions. ${ }^{117}$ Whereas hydrogen bonding is confirmed in $\mathrm{CH}_{2} \mathrm{Cl}_{2}$ and 2,2,2-trifluoroethanol (TFE), the ligand 10 is fully dissociated in $\mathrm{MeOH}$ since the 2-hydroxypyridine tautomeric form predominates in this solvent. For the asymmetric hydrogenation of $\mathbf{2 6}$ (formula in Fig. 6), with a chiral phosphepine ligand coupled to a pyridone unit, this observation explains the better enantioselectivity obtained when TFE is used as a solvent 15 instead of $\mathrm{MeOH}$ (95\% and $64 \%$ ee respectively).

In 2003, the hydrogen-bonded ligand 16-17 was reported to be very active and selective for the hydroformylation of terminal alkenes. ${ }^{56}$ Very recently an in-depth mechanistic study was provided for this reaction. ${ }^{136}$ High-pressure infrared (HP-IR) 20 analyses identified $\left[\mathrm{Rh}(\mathrm{H})(\mathbf{1 6}-\mathbf{1 7})(\mathrm{CO})_{2}\right]$ as the catalytic resting state of the transformation. In this complex, the assembled ligand behaves as in $\mathbf{1 8}$ since a fast tautomerization process is observed. The loss of one molecule of $\mathrm{CO}$ generates the active species $[\mathrm{Rh}(\mathrm{H})(\mathbf{1 6}-\mathbf{1 7})(\mathrm{CO})]$ in which the phosphine ligands are 25 approximately trans ( $\mathrm{P}-\mathrm{Rh}-\mathrm{P}=154^{\circ}$, DFT optimised geometry). The bite angle changes from $112^{\circ}$ (resting state) to $154^{\circ}$ (active species) highlighting the flexible character of this assembled ligand. This flexibility is probably at the origin of the unique catalytic properties of this ligand system since it can adopt various 30 coordination geometries and bite angles without severe energy penalties. More experimental and computational data are provided which confirm that hydrogen bond interactions between $\mathbf{1 6}$ and $\mathbf{1 7}$ are maintained during the whole catalytic cycle. The encountered flexibility requirement agrees very well with the findings for 35 covalent diphosphines in rhodium-catalysed hydroformylation, as not all ligands coordinating in an equatorial-equatorial fashion lead to high l:b ratios. ${ }^{28}$ A self-assembled ligand, derived from 1617, was used for the rhodium-catalysed hydroformylation of internal and terminal aryl alkynes providing $E$-enals with high ${ }^{40}$ selectivity. ${ }^{137}$

With Pd, the hydrogen-bonded ligand 16-17 catalysed the allylation of $N$-heterocycles with allylic alcohols (13 examples, $33 \%<$ yield $<96 \%$ ). ${ }^{123}$ Even though the mechanism is not clearly established, hydrogen bond interactions between a "free" $\mathrm{N}-\mathrm{H}$ 45 proton of 16-17 and the hydroxyl group of the substrate are proposed that makes the hydroxyl group a better leaving group (see Fig. 41, section 5.1.2.1). For the ruthenium-catalysed hydration of nitriles, a similar interaction between the hydrogen-bonded ligand and an incoming water molecule seems plausible (see Fig. 34, 50 section 5.1.2.1). ${ }^{122}$ Clearly, with a given supramolecular bidentate ligand, the nature of the interaction (ligand - ligand and/or ligand - substrate interactions) depends on the reaction studied.

2-Amidopyridine and isoquinolone are self-complementary motifs which mimic the hydrogen bonding pattern of the 55 adenine-thymine DNA pair. Phosphane ligands were prepared which combine the 2-amidopyridine or the isoquinolone groups and the ligating phosphorus atom in the same covalent skeleton. Mixing phosphane ligands that possess self-complementary motifs with a transition-metal only gives the hetero-complex. ${ }^{111}$ Ligand ${ }_{60}$ heterocombinations prove to be more efficient than the respective ligand homocombinations in a variety of catalytic reactions. ${ }^{10,111,120-126}$ For example, this ligand self-assembly strategy enables the easy preparation of ligand libraries and their screening in rhodium-catalysed asymmetric hydrogenation 65 reactions. ${ }^{120,126}$ Wieland et al. prepared 12 ligands with the 2-amidopyridine or 2-aminothiazole motifs and 10 ligands with the isoquinolone or azaindole complementary groups (Fig. 6). ${ }^{126}$ With this $12 \times 10$ ligand library in hand, the 120 catalysts were screened by an iterative deconvolution strategy. Indeed, instead of 70 evaluating each catalyst independently, the ligands are divided in subgroups and the catalysts obtained by mixing each subgroup are evaluated together. The ligand subgroup which gives the best result in terms of activity and selectivity is selected and is deconvoluted further by division into new subgroups. In the last iterative step, 75 each subgroup only contains one ligand and the catalytic results reveal the nature of the best catalyst. Here, for the asymmetric hydrogenation of MAA (10) only 17 reactions were needed to identify three efficient catalysts (19, 20 and 21, Fig. 6). A parallel screening of the 120 catalysts validated the iterative deconvolution 80 strategy because the same catalysts were identified as the best ones in both cases. With four other substrates (25-28), very active catalysts were found but the nature of the best ligand heterocombinations differs in every case ( 20 for $\mathbf{2 5 ,} 22$ for $\mathbf{2 6}, 23$ for 27 and 24 for 28, Fig. 6). Ligand heterocombinations always 85 provide higher enantioselectivity than ligand homocombinations, proving that the former are the active species. Although ligandligand interaction plays an obvious role in the catalytic performance by furnishing highly selective heterocombinations, ligand - substrate interactions cannot be ruled out on the basis of 90 the provided data.

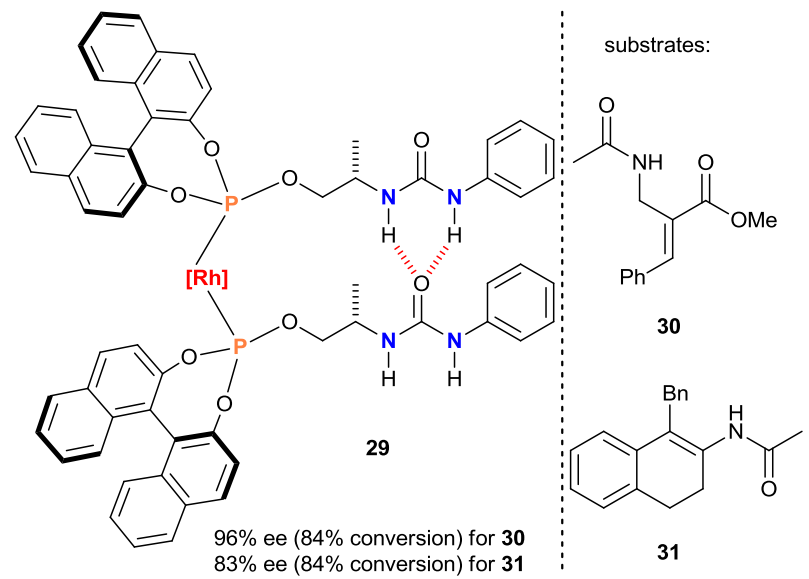

Fig. 7 1,3-disubstituted UREAPhos as self-assembled ligands for asymmetric hydrogenation. Ref: see the text.

Coordination chemistry studies involving some members of the ${ }_{95}$ UREAPhos family showed that one acidic urea proton is readily deprotonated in presence of metal precursors such as $\left[\mathrm{Rh}(\mathrm{acac})(\mathrm{CO})_{2}\right]$; complexes are formed where the UREAPhos ligands are linked by a bridging hydrogen atom (see Fig. 4). ${ }^{104,105}$ In striking contrast, the reaction of two equivalents of UREAPhos 100 ligands with a "neutral" rhodium precursor, such as 


\section{Cite this: DOI: $10.1039 / \mathrm{c0xx00000x}$}

\section{www.rsc.org/xxxxxx}

ARTICLE TYPE

$\left[\mathrm{Rh}(\mathrm{nbd})_{2}\right] \mathrm{BF}_{4}$, always yields the complex with the doubly hydrogen-bonded bidentate ligand. ${ }^{104,129,130}$ An automated procedure was followed for the fast evolution of a small library of UREAPhos ligands in the asymmetric hydrogenation of 5 industrially relevant substrates. ${ }^{130}$ Twelve ligands were prepared which differ by the nature of the linker between the urea function and the phosphorus atom and by the chiral substituent(s) located on the phosphorus atom. In the first screening the most promising catalyst for each substrate was identified. A second screening 10 concerned the reaction conditions required for the highest catalytic activity and selectivity. The best catalyst 29 (Fig. 7) provided $96 \%$ ee ( $84 \%$ conversion) and $83 \%$ ee ( $84 \%$ conversion) for substrates 30 and 31 respectively. NMR experiments of the precatalyst assemblies at different concentration confirm the presence of 15 intramolecular hydrogen bonds.
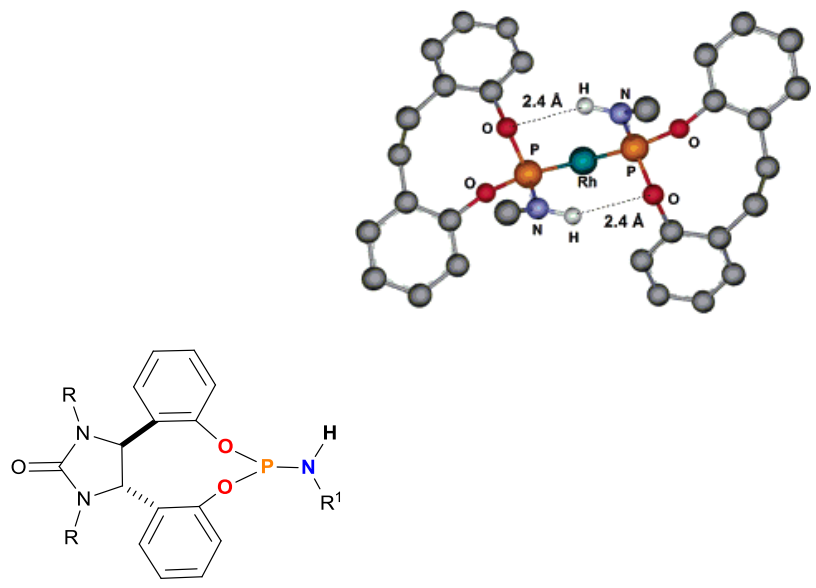

\section{DpenPhos ligands}

Fig. 8 Left: General formula of the DpenPhos ligands. Right: Optimisedstructure of a $\left[\mathrm{Rh}(\mathrm{DpenPhos})_{2}(\mathrm{cod})\right] \mathrm{X}$ complex emphasizing the hydrogen bond interactions between the two ligands. (The optimised 20 structure is reprinted with permission from ref. 132. Copyright 2006. American Chemical Society).

Liu et al. reported DpenPhos a class of monodentate ligands that self-assembled via two hydrogen bonds; the resulting bidentate ligands coordinated to Rh in a cis-position (Fig. 8). ${ }^{132}$ These chiral 25 phosphoramidite ligands proved to be very selective for the asymmetric hydrogenation of ( $Z$ )-methyl $\alpha$-(acetoxy)acrylates and (E)- $\beta$-aryl itaconate derivatives (14 examples, $94<$ ee $<99 \%$ ). In the precatalysts, the bite angle of the assembled diphosphorus ligand is of $89.9^{\circ}$, which is smaller than the one observed for a 30 structurally-related phosphoramidite ligand lacking the hydrogen bond donor $\mathrm{N}-\mathrm{H}$ group $\left(94.8^{\circ}\right)$. This hydrogen bond interaction coupled with the alteration of the ligands bite angle can explain the excellent catalytic performance exhibited by DenPhos ligands.

Breit group developed a family of peptide-derived 35 monophosphine ligands that self-assembled as a result of hydrogen bond interaction between the peptide groups. These ligands are named SupraPhanePhos because the helical hydrogen-bonding network induces a planar chiral $\pi$-stacking arrangement of the two meta-substituted arene rings (Fig. 9, a). ${ }^{133}$ With two amino acids 40 in the side chains, chirality transfer produced modest enantioselectivity in asymmetric hydrogenation (51\%). ${ }^{133}$ When supplementary chiral information is present on the phosphorus atom, the catalysts are far more enantioselective. ${ }^{133}$ Kokan et al. recently slightly improved this result by using para-substituted 45 arene rings with three amino acids in the side chains (up to $68 \%$ ee for the hydrogenation of 10). ${ }^{138}$ These results are of interest, because the chirality is derived from remote groups, and not, like the majority of the results in this review, from a chiral BINOL group. It should be borne in mind that most BINOL-derived 50 systems give modest to excellent enantiomeric excesses in the absence of supramolecular interactions.

Upon coordination of rhodium or palladium atoms, $C$-linked phosphine-functionalized peptide ligands interact with the complementary $N$-linked phosphine-functionalized peptide 55 counterparts and thus selectively form hetero-complexes (Suprapeptiphos ligands, Fig. 9, b). ${ }^{112}$ Two amino acids in the side chain are enough to direct the assembly through the formation of an antiparallel $\beta$-sheet structure as a result of three hydrogen bonds established between each monophosphine ligand. An accurate 60 study of a series of structurally-related ligands allows the determination of the structural factors that are crucial for the assembly. ${ }^{108}$ Interestingly, hydrogen bonds are not the only contributors to the overall complex stability, because a weak $\mathrm{C}$ $\mathrm{H} \cdot \cdots \mathrm{N}$ hydrogen bond, participates in the stabilisation of the ${ }_{65}$ hetero-complex. Such an interaction is present both in solution and in the solid state (see the PLATON plot of the solid state structure of complex 35, Fig. 9, b). As a result, the hetero-complex 32, which is stabilised by a weak hydrogen bond, is far much favoured over the respective homo-complexes than the hetero-complexes $\mathbf{3 3}$ 70 and 34 which lack this interaction. A library of Suprapeptiphos ligands was evaluated in the asymmetric hydroformylation of styrene $(5.7<\mathrm{b}: 1 \quad$ ratio $<19)$ for which some ligand heterocombinations proved to be more efficient than the respective ligand homocombinations. ${ }^{112}$ Selectivity was low $(38 \%$ ee in the 75 best case) but again it should be kept in mind that chirality was located on the remote peptide side chains. ${ }^{139-141}$

It must be stressed that for all the systems mentioned above hydrogen bonding was found to be primordial for the observed catalytic efficiency. However, the exact role of the hydrogen bond 80 interaction was not always carefully probed. The following example highlights how the nature of the selectivity-determining interaction can be properly assigned. Pignatoro et al. prepared chiral monodentate phosphite ligands, named PhthalaPhos, which contain a phthalic acid primary amid moiety (Fig. 10). ${ }^{134}$ ${ }_{85}$ PhthalaPhos ligands exhibit higher selectivity for the asymmetric hydrogenation of substrates $\mathbf{1 0}, \mathbf{2 6}, \mathbf{2 7}, \mathbf{2 8}$, and 37 than a chiral monophosphite ligand taken as a reference. NMR studies and DFT calculations confirmed the presence of two intramolecular hydrogen bonds between the $\mathrm{N}-\mathrm{H}_{\mathrm{A}}$ protons and the carbonyl 90 oxygens of the PhthalaPhos ligands in the rhodium precatalysts 
(N-HB remaining free, Fig. 10). However, control experiments 5 methylated substrates gave lower conversion and selectivity.

with $N$-Me analogue of $\mathbf{3 6}$ indicated that the same selectivity $(96 \%$ ee) and activity were obtained with this ligand for the hydrogenation of substrate 37. Additionally, hydrogenation of $\mathrm{N}-$

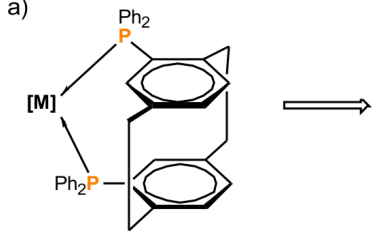

PhanePhos planar chiral

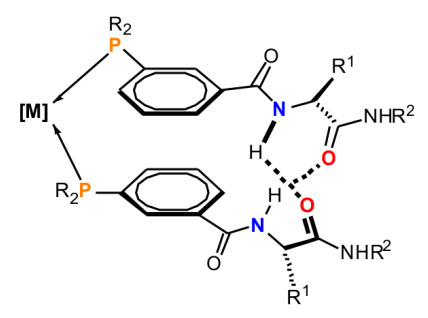

SupraPhanePhos central to planar chirality induced through self-assembly

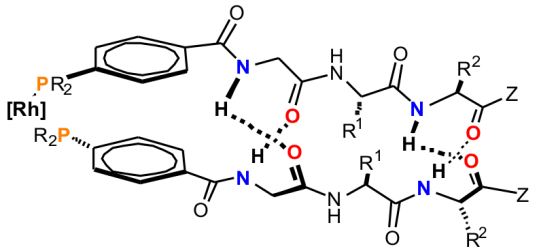

Kirin's group, 2012

b)

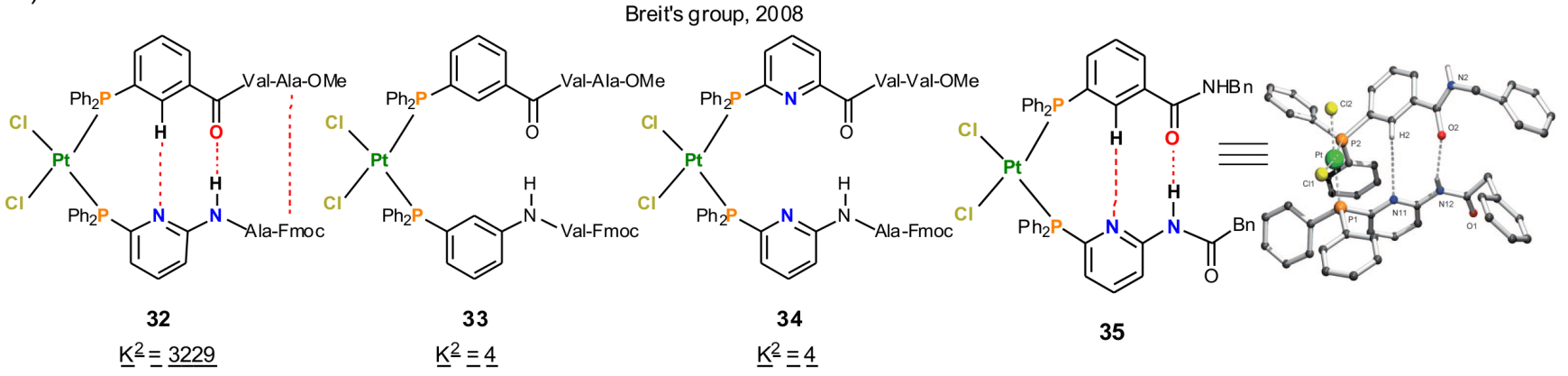

Fig. 9 SupraPhanePhos and Suprapeptiphos ligands. (a) General formula of PhanePhos and SupraPhanePhos ligands. (b) Cooperative effect of classical 10 and weak hydrogen bonds for the metal-induced construction of hetero-complex 32.PLATON plot of the solid state structure of complex 35.K constant reflects how much the formation of the hetero-complex is enhanced compared to a statistical distribution. $\mathrm{K}=[\mathrm{Hetero}]^{2} /[\mathrm{Homo}] \mathrm{x}[\mathrm{Homo}] ; \mathrm{K}=4 \mathrm{for}$ a statistical distribution. (PLATON Plot is reprinted with permission from ref. 108. Copyright 2009. John Wiley and Sons). Refs: see the text.

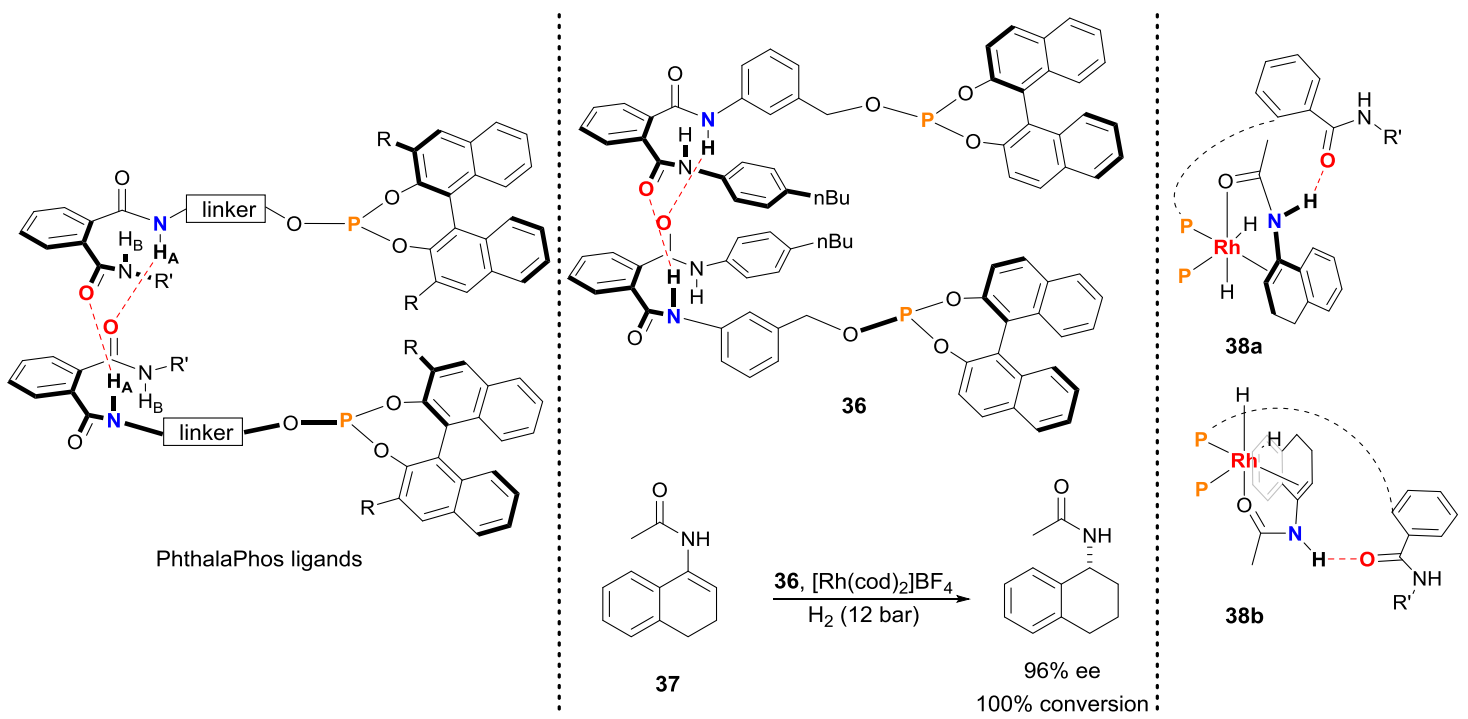

Fig.10 PhthalaPhos ligands. Left: General structure of the PhthalaPhos ligands. Middle: Best catalyst for the asymmetric hydrogenation of 37. Right: Representation of the hydrogen bond between the carbonyl oxygen of the $\mathbf{3 6}$ ancillary amide group and the N-H proton of substrate $\mathbf{3 7}$ in $\mathrm{Si}$ -

$\left[\mathrm{Rh}(\mathrm{H})_{2}(\mathbf{3 7})(\mathbf{3 6})\right]^{+}$as observed in DFT-optimised structures (38a is $14.8 \mathrm{kcal}^{\mathrm{mol}}{ }^{-1}$ more stable than 38b). Ref: see the text.

Clearly, the catalytic efficiency of PhthalaPhos ligands is governed by ligand - substrate rather than ligand - ligand interactions. 20 Indeed, DFT calculations confirmed that that the intramolecular hydrogen bonding between PhthalaPhos ligands presents in the precatalyst is lost during the catalytic cycle at the expense of ligand
- substrate interactions (hydrogen bond established between the $\mathrm{N}-\mathrm{H}$ proton of the substrate and the carbonyl oxygen of the 25 ancillary amide group of one PhthalaPhos ligand, see 38a and 38b, Fig. 10). ${ }^{135}$ This can be a general trend of this family since related ligands with a benzamide residue (instead of phthalamide) behave 


\section{Cite this: DOI: $10.1039 / \mathrm{c0xx00000x}$}

similarly. ${ }^{142}$

Most of the examples reported so far apply supramolecular interactions for the assembly of two monophosphine ligands. Durini et al. reported Suprabox: a series of chiral urea-oxazoline

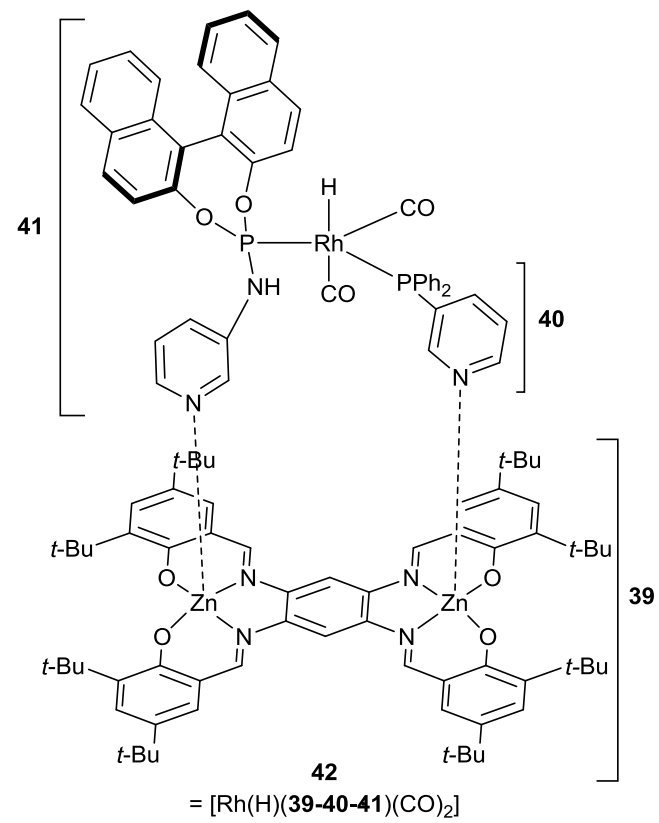

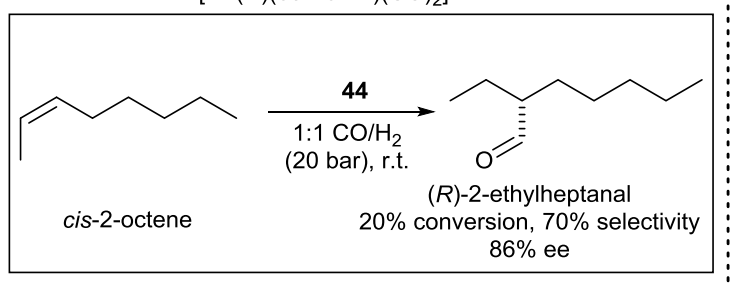

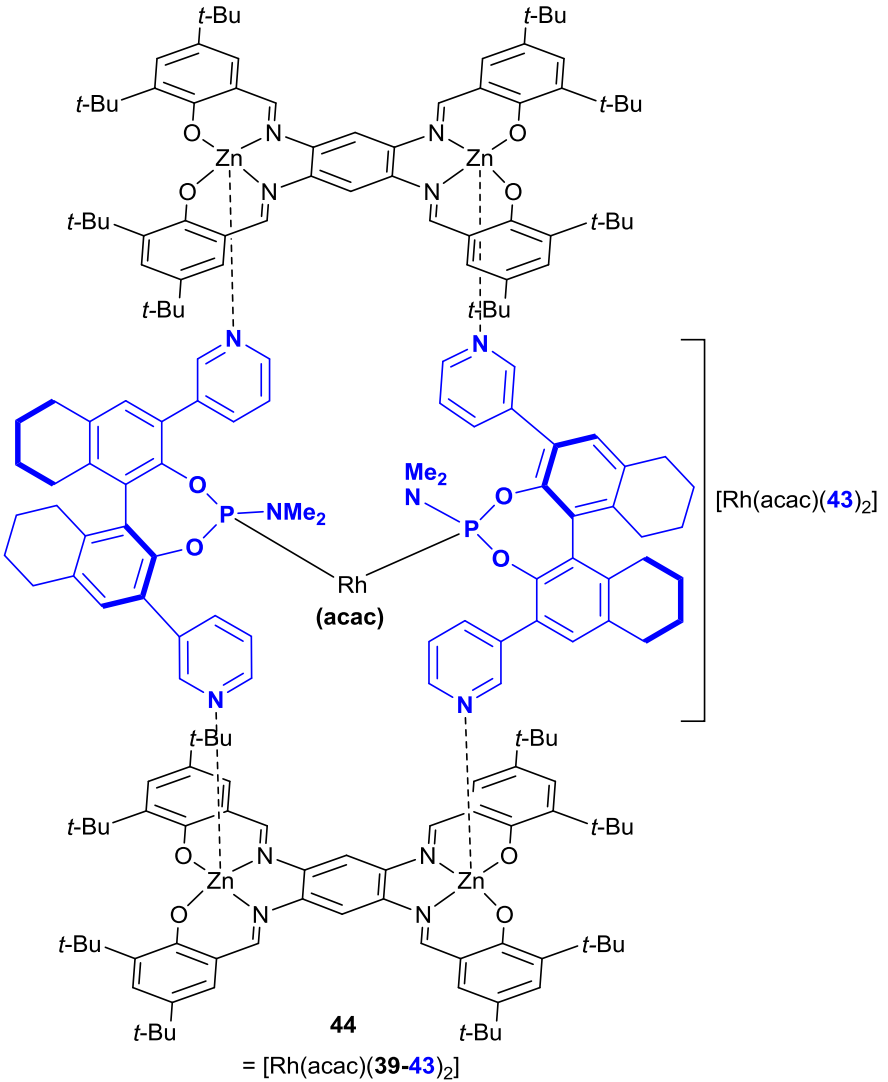

Fig. 11 Use of Zn-bis(salphen) platforms as a template for the assembly of two pyridylphosphane ligands. Left: 39 used as a template for the selective formation of the hetero-complex $\mathbf{4 2}$. Selective hydroformylation of cis-2-octene with precatalyst $\mathbf{4 4}$. Right: Formula of precatalyst $\mathbf{4 4}$, formed by combination of 39, $\mathbf{4 3}$ and $\left[\mathrm{Rh}(\mathrm{acac})(\mathrm{CO})_{2}\right]$. Refs: see the text.

ligands. ${ }^{131}$ Coordination chemistry studies confirmed the 10 formation of bis(oxazoline) copper and palladium complexes. Good selectivities were obtained for the copper-catalysed benzoylation of vic-diols. Kinetic resolution of racemic hydrobenzoin (up to $86 \%$ ee and selectivity $(s)=28$ ) and desymmetrization of meso-hydrobenzoin (up to $88 \%$ ee) was

15 accomplished. A covalent Box analogue gave $99 \%$ ee in the reaction of rac-hydrobenzoin, but, more importantly, the related oxazoline monomer without hydrogen bonding functionalities gave $0 \%$ ee.

3.1.3 Lewis acid - Lewis base and ionic metal-ligand 20 interactions

In this subsection, we describe examples of the use of a metal as template for the assembly of two ditopic ligands.

Van Leeuwen and Reek first used Lewis acid - Lewis base interactions for the construction of supramolecular bidentate 25 ligands. Two Zn-porphyrin platforms, linked by a covalent spacer, were used as a template for the association of two pyridylphosphanes (such as 40 and 41, Fig. 11). Pyridylphosphines alone and their complexes with a Zn-monoporphyrin platform served as monophosphine references. High-pressure NMR 30 analyses confirmed the formation of $(P, P)$-chelates. This was corroborated by the catalytic results; the supramolecular bidentate ligands exhibited higher selectivity in alkene hydroformylation and allylic alkylation reactions compared to references. ${ }^{143}$ One of the advantages of this system is that the formation of hetero35 complexes is entropically driven. Higher rates and selectivities were obtained when heterocombinations of pyridylphosphines, pyridylphosphoramidites or pyridylphosphites were used in combination with bis $\mathrm{Zn}$-bis(salphen) platform 39 (Fig. 11 left). ${ }^{144}$ HP-NMR and HP-IR studies established that the hetero-complex 40 42, with the pyridylphosphanes $\mathbf{4 0}$ and $\mathbf{4 1}$ coordinated to $\mathrm{Rh}$ in a equatorial-equatorial fashion, is the catalytic resting state for the hydroformylation of styrene ( $19 \%$ conversion, b: 1 ratio $=9.2,72 \%$ ee). The properties of this catalytic system were further investigated by changing the nature of the template and the 45 pyridylphosphine, and in most of the cases the bidentate assemblies showed higher selectivity than the respective 
references. However, the overall catalytic efficiency of these

reported the chiral $\mathrm{H}_{8}$-BINOL-derived phosphoramidite ligand $\mathbf{4 3}$ catalysts remained modest. ${ }^{145-147}$ Recently, Reek and co-workers

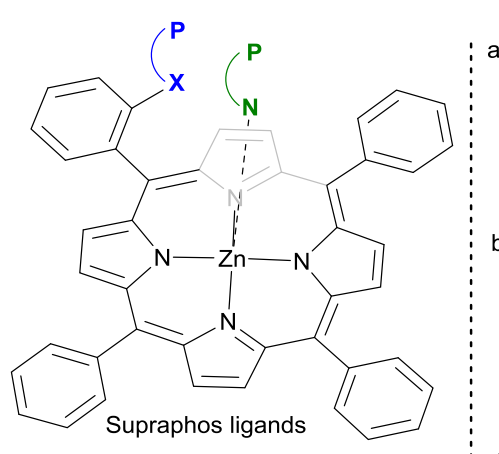<smiles>CC(=O)NC1=Cc2ccccc2CC1</smiles><smiles>[X]CCPc1ccccc1-c1ccccc1</smiles>
b)

$100 \%$ conversion, $94 \%$ ee<smiles>CC(=O)O[C@H]1C=CCC[C@H]1C(C)=O</smiles><smiles>[X]c1cccc2ccc3op(OCN)oc4ccc5ccccc5c4c3c12</smiles>
$81 \%$ conversion, $32 \%$ ee $(46), 99 \%$ ee $(47)$

c)

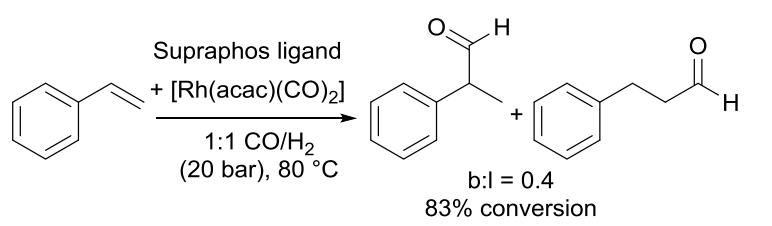<smiles>[X]CP=C</smiles><smiles>NCCPC#CCc1cccnc1</smiles>

Fig. 12 Supraphos ligands. Best ligand heterocombinations found for the rhodium-catalysed hydrogenation of the challenging enamide substrate $\mathbf{4 5}$ (a), for the palladium-catalysed kinetic resolution of racemic cyclohexenyl acetate (b) and for the rhodium-catalysed hydroformylation of styrene (c). Refs: see the text.

that possess two peripheral pyridine rings available for the 10 coordination of Zn-bis(salphen) platforms (Fig. 11 right). ${ }^{148}$ Combination of two equiv. of $\mathbf{4 3}$ and two equiv. of $\mathbf{3 9}$ led to a very robust bidentate ligand. Upon coordination to $\left[\mathrm{Rh}(\mathrm{acac})(\mathrm{CO})_{2}\right]$, the supramolecular complex 44 was formed in which the rhodium atom was confined between two units of 39. The resulting catalyst 15 exhibited an unusual selectivity during the hydroformylation of trans- and cis-2-alkenes: the innermost aldehyde was produced preferentially. In addition the $(R)$-enantiomer of this aldehyde is obtained with high optical purity. For example, the hydroformylation reaction of cis-2-octene performed at $25{ }^{\circ} \mathrm{C}$ 20 yields $(R)$-2-ethylheptanal with $86 \%$ ee, albeit at a modest $20 \%$ conversion (Fig. 11 left). The activity was increased by performing the reaction at $40{ }^{\circ} \mathrm{C}$ at a low cost of regio- and enantioselectivity. The supramolecular ligand (39-43)2 clearly outperformed the commercially available CHIRAPHITE and $(S, S)$-DIOP ligands, as 25 well as other self-assembled ligands (e.g. 83•712, Fig. 24) under the same reaction conditions. The results were explained by the steric effects exerted by the $\mathrm{Zn}$-salphen platforms within the encapsulated $\mathrm{Rh}$ catalyst.

A more elaborated self-assembled system consisting of a 30 phosphite ligand covalently attached to three Zn-porphyrin platforms was studied for the formation of a supramolecular bidentate box-like ligand. ${ }^{149}$ Three molecules of DABCO (1,4-diazabicyclo[2.2.2]octane) each one sandwiched between two porphyrin units act as supramolecular columns. The association 35 constant of the Zn-porphyrin platform with the DABCO molecules is stronger than the one with the pyridylphosphines described above. The formation of a complex with a chelating diphosphite ligand is clearly demonstrated by the result of the catalytic hydroformylation of 1-octene: a l:b ratio of 22.8 is obtained at 30 ${ }_{40}^{\circ} \mathrm{C}$.

Reek and co-workers devised a supramolecular approach for preparing bidentate ligands by simply mixing two monomeric phosphorus-based ligands each of which incorporate complementary group: a Zn-porphyrin platform or a pyridyl donor.

45 This methodology has enabled efficient preparation of libraries of structurally-diverse bidentate ligands called Supraphos with unprecedented ease (Fig. 12). The selective binding of pyridyl donors to the zinc acceptors, in addition to a chelate effect (7 $\mathrm{kJ} . \mathrm{mol}^{-1}$ ), are the driving forces for the formation of stable hetero50 complexes with $(P, P)$-chelate ligands. This strategy constitutes a powerful tool that has led in some cases to unprecedented catalytic activity for the catalysts derived from Supraphos ligands (Fig. 12, a-c). For the hydrogenation of the challenging acetamide substrate 45 (Fig. 12, a) the best ligand heterocombination outperformed ${ }_{55}$ BINAPHOS $^{150,151}$ a covalent bidentate phosphine-phosphite ligand that has been applied successfully to many asymmetric transformations. ${ }^{152}$ The large aromatic surface of the porphyrin molecule likely played an important role by creating a pocket that directs the substrate. For the palladium-catalysed kinetic resolution 60 of racemic cyclohexenyl acetate, the best Supraphos ligand exhibits a remarkable activity (Fig. 12, b). It yields high ee of the $S$ enantiomer of cyclohexenyl acetate above conversions of $60 \%$ but the ee of the product is low (30\%) and opposite. ${ }^{153}$ This unusual behaviour can be related to the dynamic character of the Supraphos ${ }_{65}$ ligand. In the hydroformylation of styrene, subtle structural modification of one of the components had a great impact on the catalytic result (Fig. 12, c). ${ }^{154}$ The rhodium catalysts derived from various chiral Supraphos ligands provide an unusual selectivity for this reaction: the linear aldehyde is formed preferentially (b:1 ratio ${ }_{70}=0.4$ ). This regioselectivity is unprecedented for a phosphoramidite-phosphine bidentate ligand and its origin remains unclear. The authors proposed that $\pi-\pi$ interactions between the aromatic groups of the Supraphos ligand and the styryl group of the substrate may slow down the migratory insertion of $\mathrm{CO}$ and 75 facilitate $\beta$-hydride elimination in the branched intermediate. ${ }^{155}$ Goudriaan et al. investigated a library of 437 chiral Supraphos 


\section{Cite this: DOI: $10.1039 / \mathrm{c0xx00000x}$}

\section{wWw.rsc.org/XXXXXX}

ARTICLE TYPE

ligands for the rhodium-catalysed

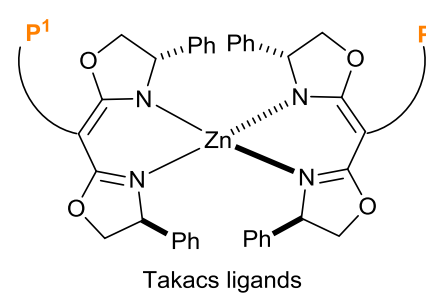

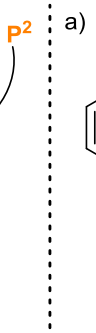

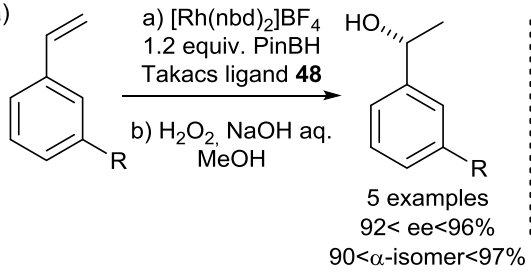

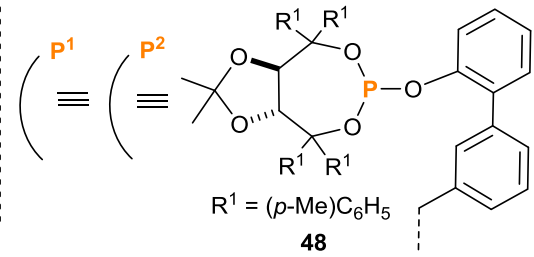

b)

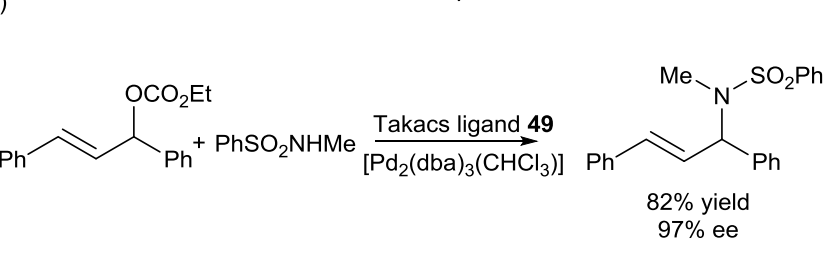

c)<smiles>C#CCCCCc1cccc(COp2oc3ccc4ccccc4c3c3c(ccc4ccccc43)o2)c1</smiles>

Fig. 13 Takacs ligands. Best ligand combinations found for the rhodium-catalysed asymmetric hydroboration of $m$-substituted styrenes (a), for the palladium-catalysed asymmetric amination of 1,3-diphenylallyl-ethyl carbonate (b) and for the rhodium-catalysed asymmetric hydrogenation of substrate $\mathbf{5 0}$ (c). In the latter case, diastereomeric ligands $\mathbf{5 1}$ and $\mathbf{5 2}$ that only differ by their configuration at the $\mathbf{Z n}$ metal centre, give different selectivity. Refs: see the text.

hydroformylation of styrene: the branched isomer was formed 10 preferentially but with very low optical purity. ${ }^{154}$ Chelate-assembled Supraphos ligands have behaved as highly efficient bidentate ligands in the above mentioned transformations for a wide array of substrates, although in a few specific cases, the non-assembled monodentate Supraphos ligand surprisingly

15 outperformed the assembled bidentate ligand in terms of enantioselectivity. ${ }^{156,157}$

Takacs et al. first reported the use of anionic metal-ligand interactions for the construction of bidentate supramolecular ligands ${ }^{158}$ not counting the numerous, older publications in the area 20 of "bimetallic" catalysis in which also most often one metal functions as the assembly metal and the other one is the catalytic metal. ${ }^{159-164}$ The synthetic route of Takacs ligands involved self-assembly of two anionic N,N bis(oxazoline) (bis(4,5-dihydrooxazol-2-yl)methane) ligands around a $\mathrm{Zn}^{\mathrm{II}}$ ${ }_{25}$ centre, acting as the metal template (Fig. 13). Apart of a bis(oxazoline) group for Zn-directed assembly, each fragment contained a conformationally labile aryl-based linker and a second set of ligating groups (i.e. TADDOL-derived phosphite groups), equipped to bind a second metal centre and to form a catalytic site.

30 The self-assembly process showed high levels of self-sorting, i.e. starting from a 1:1 mixture of $(R, R)$ - and $(S, S)$-bis(oxazoline) only the $\mathrm{Zn}^{\mathrm{II}}$ hetero-complex is formed which contains one $(R, R)$ - and one $(S, S)$-bis(oxazoline) ligand. ${ }^{165-167}$ In other words, in the Takacs system, the reaction between two different monophosphane 35 ligands attached to bis(oxazoline) units with opposite configuration and a $\mathrm{Zn}^{\mathrm{II}}$ precursor yields quantitatively the ligand heterocombination. Varying the ligand scaffold (linker and/or ligating groups) enabled easy generation of ligand libraries, from which highly efficient enantioselective catalysts were identified. 40 Analysis of the hits for three reactions, palladium-catalysed asymmetric allylic amination, ${ }^{158}$ rhodium-catalysed hydrogenation $^{167,168}$ and 


\section{Cite this: DOI: 10.1039/c0xx00000x}

\section{www.rsc.org/XXXXXX}

ARTICLE TYPE
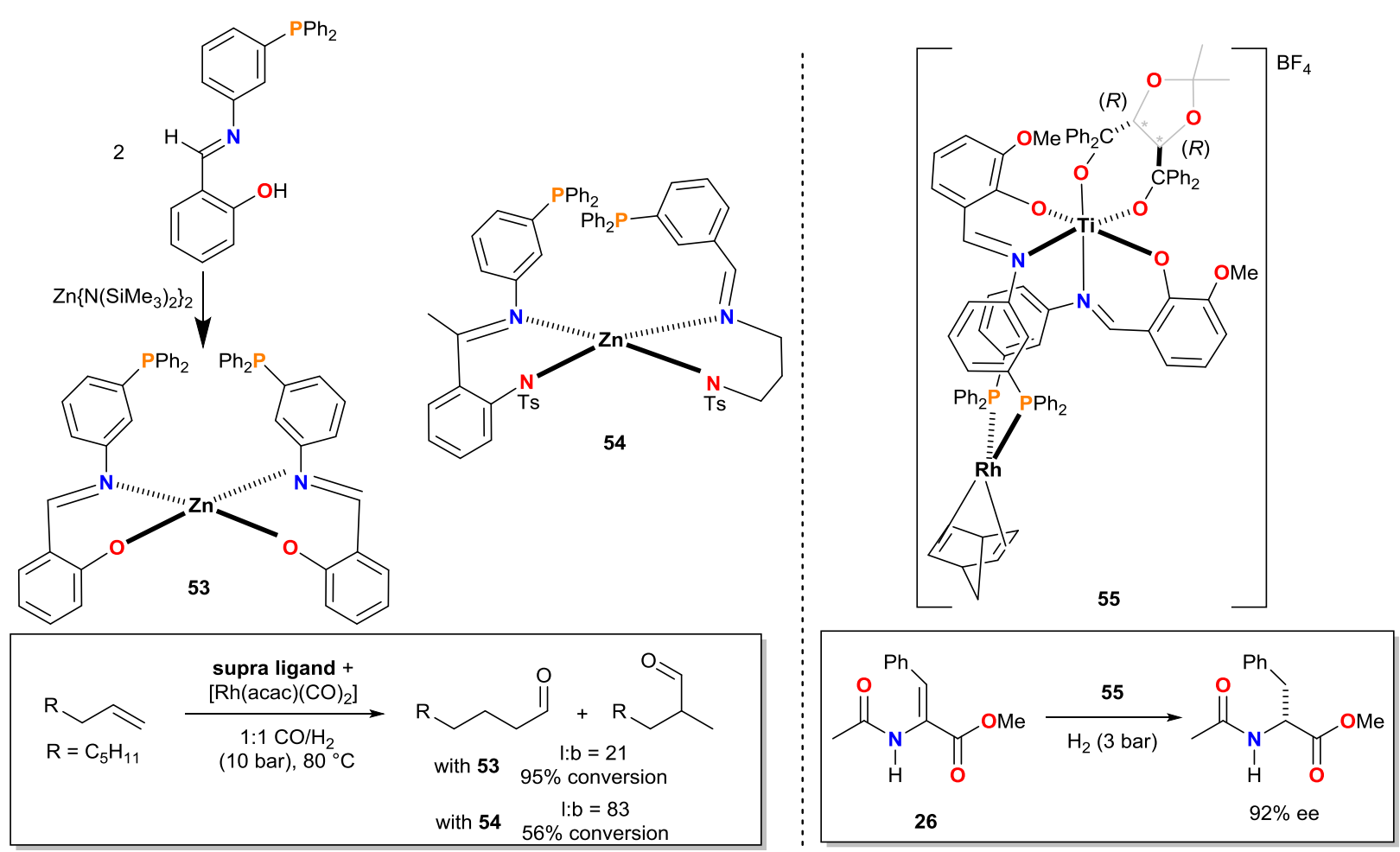

Fig. 14 Construction of supramolecular bidentate ligands by means of anionic metal-ligand interactions. Left: The rhodium catalysts containing the bidentate ligands $\mathbf{5 3}$ and $\mathbf{5 4}$ gave preferentially octanal as the hydroformylation product of 1-octene. Right: Possible structure for the self-assembled precatalyst $\mathbf{5 5}$ that possesses a remote chiral inducer. Catalyst derived from $\mathbf{5 5}$ hydrogenates $\mathbf{2 6}$ selectively ( $92 \%$ ee). Refs: see the text.

5 rhodium-catalysed hydroboration ${ }^{169,170}$ revealed that different sets of hits were obtained (Fig. 13). Takacs ligand $\mathbf{4 8}$ possess identical linkers between the phosphite ligating groups and the bis(oxazoline) recognition sub-units. The rhodium-catalyst derived from 48 (Fig. 13, a) provided benzylic alcohols with high optical 10 purity from meta-substituted styrenes. ${ }^{170}$ Spectroscopic studies and DFT calculations confirmed that the precatalyst is a $\mathrm{Rh}, \mathrm{Zn}$ dinuclear complex with a cis-arrangement of the phosphorus atoms bound to the catalytic rhodium atom. Previously, heterobidentate ligand 49, again with a chiral $(R, R)$-TADDOL phosphite ligating 15 group, gave the best result for the palladium-catalysed amination of 1,3-diphenylallyl-ethyl carbonate (Fig. 13, b). ${ }^{158}(R)$-BINOL phosphites proved to be superior to $(R, R)$-TADDOL and $(R)$ BIPHEP phosphites for the asymmetric hydrogenation of substrate 50 (Fig. 13, c). ${ }^{168}$ The same trend is observed when the $(R)$-BINOL 20 phosphite is incorporated into Takacs ligands. The impact of each sub-unit of the assembled ligand on the catalytic performance was scrutinized and, as expected, the nature of the linker between the ligating group and the bis(oxazoline) unit plays a major role. More surprisingly, the chirality around the $\mathrm{Zn}$ template also has an 25 importance. For example, diastereomeric ligands $\mathbf{5 1}$ and $\mathbf{5 2}$ give a slightly different selectivity, indicating a matched/mismatched relationship between the chirality located on the $\mathrm{Zn}$ and the one present on the phosphorus atom. Further investigation is needed to rationalize this result, but initial assumptions are that the 30 recognition sub-units: i) influence the organisation of the linkers (and thus the overall structure of the catalyst), ii) directly interact with the substrate during the course of the reaction. Probing this potential ligand - substrate interaction is of importance for the design of catalysts with unusual selectivity.

35 Although Takacs metal-directed assembling strategy leads selectively to stable hetero-complexes by virtue of the design of the recognition sub-units, the synthesis of the monomeric ligands is not straightforward (a multistep synthetic procedure is required). Van Leeuwen and co-workers devised a Ti- or Zn-directed 40 assembly process of simpler ditopic monomeric components (twostep synthesis), which contained an $o$-hydroxy Schiff base moiety for the assembly process and a ligating-phosphorus atom for catalysis The nitrogen and oxygen atoms of the $o$-hydroxy Schiff base moiety chelate the metal centre acting as the template (i.e. 45 zinc or titanium), and the phosphorus atoms of the two monomeric units come close in space in an arrangement that is suitable for the coordination to the catalytic metal centre (Fig. 14). ${ }^{171} \mathrm{X}$-ray structures of the assembled ligands induced by $\mathrm{Zn}^{\mathrm{II}}$ coordination showed variable P-P distances (between 6.5 and $8 \AA$ ) depending 50 on the ditopic ligand 


\section{Cite this: DOI: $10.1039 / \mathrm{c0xx00000x}$}<smiles>O=[N+]([O-])c1cccc(P(c2cccc(S(=O)(=O)[O-])c2)c2cccc(S(=O)(=O)[O-])c2)c1</smiles>

TPPTS

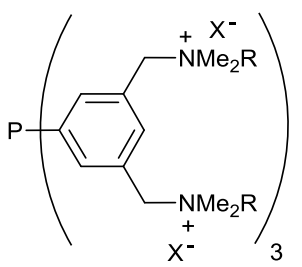

Dendriphos ligands

$\mathrm{R}=\mathrm{Me}, \mathrm{Bn}, \mathrm{G} 1$ or $\mathrm{G} 2$ Fréchet wedge b)

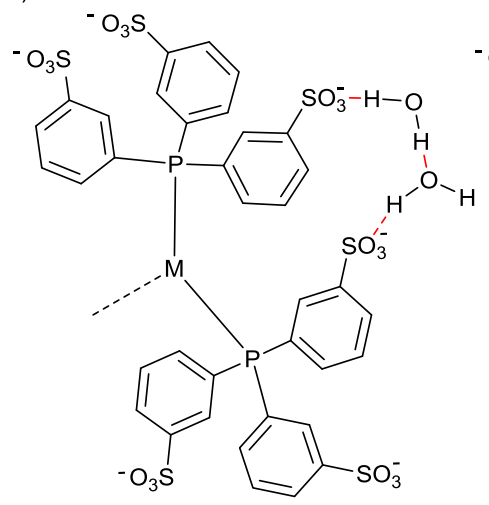

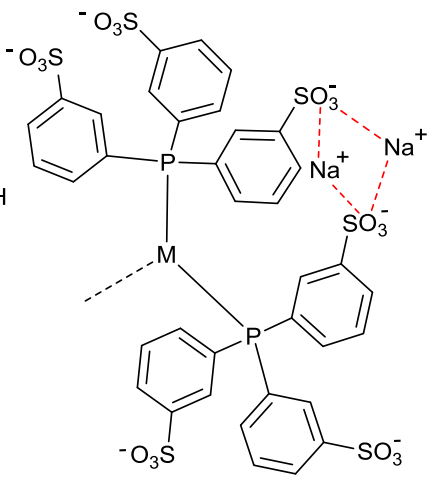

c)

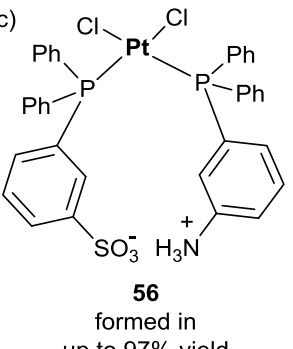

Fig. 15 Self-assembly of ionic phosphines by means of electrostatic interactions. a) Formulas of TPPTS ( tri( $m$-sulfonatedphenyl)phosphine) and Dendriphos ligands. b) Self-assembly of TPPTS ligands through hydrogen bond interactions (with bridging water molecules) or electrostatic interactions.

The model has been proposed for several catalytic reactions. c) The electrostatic interaction between mono-sulfonated triphenylphosphine and monoammonium triphenylphosphine led almost exclusively to the formation of the hetero-complex. Refs: see the text.

employed. MM2 calculations indicated the preference of a natural bite angle of approximately $110^{\circ}$ in the zinc-templated supramolecular ligands coordinated to Pd or Rh metal centres. HPIR analysis under $\mathrm{CO}$ atmosphere confirmed the preferential 10 formation of rhodium complexes $\left[\mathrm{Rh}(\mathrm{H})(\mathbf{5 3})(\mathrm{CO})_{2}\right]$ with an equatorial-equatorial arrangement of the phosphine-binding groups. ${ }^{172}$ As a result, utilization of these rhodium complexes in hydroformylation of 1-octene as substrate led preferably to the linear aldehyde (l:b ratio of 21 for the catalyst derived from $\mathbf{5 3}$, 15 Fig. 14 left). Combining different ditopic ligands gave variable mixtures of homo- and heterocombinations of the monomeric units. Evaluation of expanded ligand libraries, including ligand heterocombinations, reveals an even more selective catalyst with a l:b ratio up to 83 but at lower conversion (54, Fig. 14 left). ${ }^{172}$ ${ }_{20}$ Replacing $\mathrm{Zn}$ by $\mathrm{Ti}$ gives access to two supplementary coordination sites on the metal centre used as the template metal. A multi-component catalytic system composed of one chiral diol as the chiral inducer, a titanium centre acting as template metal, two ditopic monomeric units, and one rhodium centre as the 25 catalytic site was investigated in the asymmetric hydrogenation of benchmark substrates. ${ }^{173}$ In this case, the stereogenic centres are located in a fragment coordinated to the metal template at approximately $13 \AA$ from the double bond of the substrate (MM2 calculations). However, the chiral information at such a remote 30 position is successful transferred to the catalytic site, since the hydrogenation product of $\mathbf{2 6}$ is obtained in a $92 \%$ ee (Fig. 14 right). Control experiments confirmed that the effective catalyst is a selfassembled complex containing the four components already mentioned (chiral diol, titanium centre, ditopic monomers, 35 rhodium centre). NMR analyses of the assembled pre- catalyst $\mathbf{5 5}$ showed the formation of a major well-defined $C_{1}$-symmetric rhodium complex with the diphosphine unit coordinated in the cis fashion (other diastereoisomers of $\mathbf{5 5}$ were observed in trace amounts). The coordination mode around $\mathrm{Ti}$ has $\mathrm{C}_{2}$ symmetry, as 40 drawn, but the resulting complex has a lower symmetry; details are not known. The mechanism of the chirality transfer is not established either; at this time none specific interaction between the ligand backbone and the substrate is envisaged.

Even though ditopic ligands were used in the past for the 45 construction of homo- or heterobimetallic complexes, ${ }^{162}$ Takacs and van Leeuwen systems constitute the first examples of efficient catalysts based on a ligand scaffold that can be easily modified. ${ }^{159-}$ 164

\subsubsection{Other type of interactions}

50 In a few cases, weak attractive interactions (such as van der Waals, $\mathrm{C}-\mathrm{H}-\pi$ or $\pi-\pi$ interactions) between the ligands have been detected as the factor responsible for an excess formation of heterocomplexes. ${ }^{174,175}$ In catalysis, such a bias from the statistical distribution is desirable when the hetero-complexes are more 55 active than the respective homo-complexes. Hydrogen bond and metal-ligand interactions are to date the most used interactions for the construction of supramolecular bidentate ligands. However, other types of attractive interactions (electrostatic, ionic hydrogen bond, hydrophobic, $\pi-\pi$ stacking) can be used to design catalysts 60 with self-assembled ligands. ${ }^{176-178}$ The number of coordinated anionic or cationic ligands, such as TPPTS and Dendriphos ligands respectively (Fig. 15, a), in homoleptic transition-metal complexes is usually governed by Coulombic interligand repulsion. ${ }^{179}$ This interaction, between coordinated 65 ionic ligand and between free ionic ligand and the metal complex, tends to limit the number of ionic ligands coordinated to the metal centre. ${ }^{180}$ In palladium-catalysed 


\section{Cite this: DOI: $10.1039 / \mathrm{c0xx00000x}$}

a)

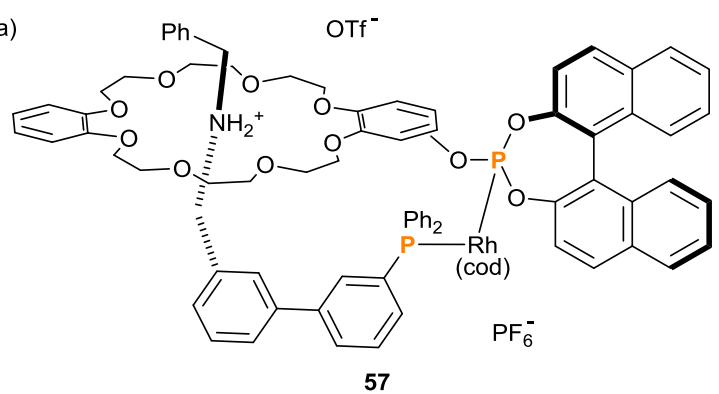

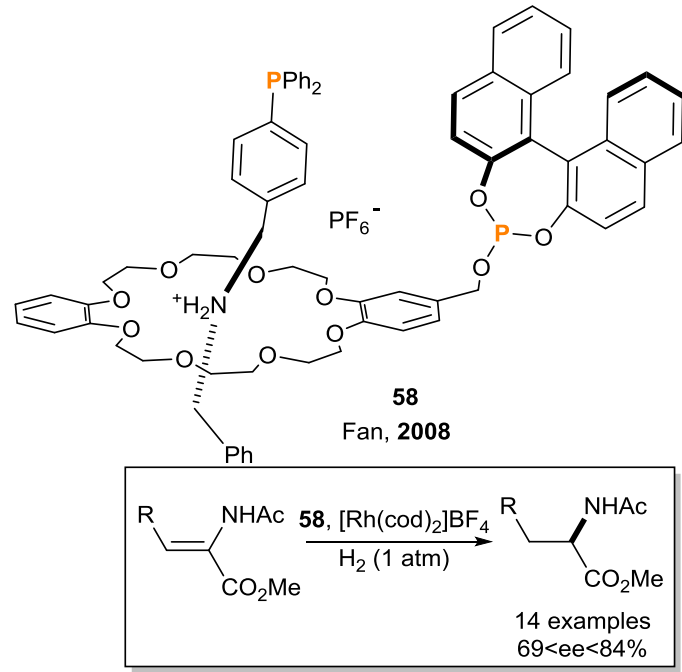

b)
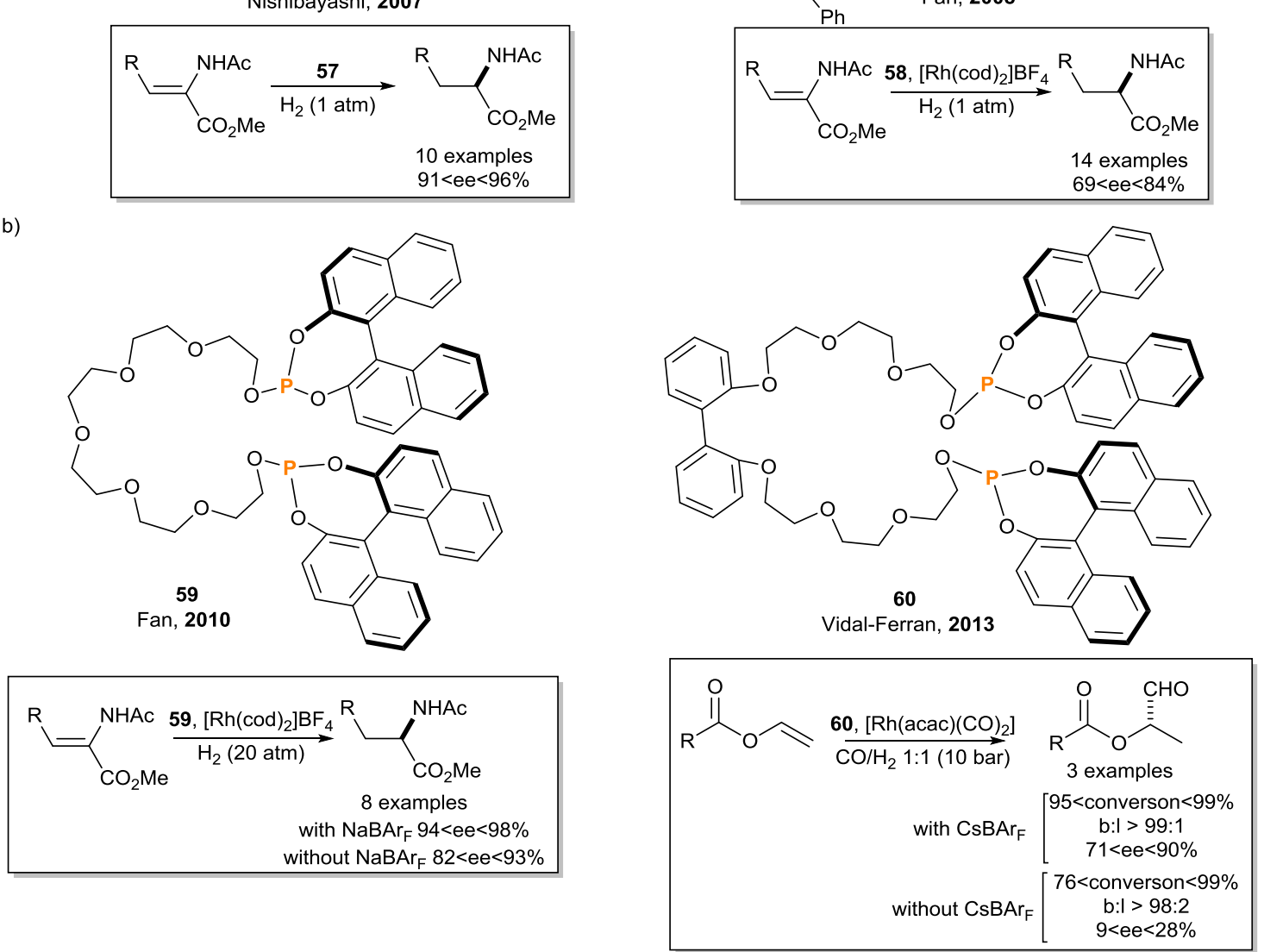

Fig. 16 Formation of supramolecular bidentate ligands by means of cation-dipole interactions. (a) Chiral ligands $\mathbf{5 7}$ and $\mathbf{5 8}$ are based on a pseudorotaxane skeleton. (b) Coordination of an alkali metal to the poly(ether) backbone of $\mathbf{5 9}$ and $\mathbf{6 0}$ enforces the formation of chelate rhodium complexes. Refs: see the text.

${ }_{5}$ Suzuki-Miyaura cross-coupling reactions with Dendriphos ligands, ${ }^{181}$ the Coulombic interligand repulsion favours the formation of homogeneous monoligated-Pd(0) species. ${ }^{182,183}$ On the contrary, non-covalent interactions can also be used to stabilise metal complexes that contain two or more coordinated ionic 10 ligands. For example, in the rhodium-catalysed hydroformylation of linear alkenes in water, ${ }^{184}$ active species with TPPTS ligands are less active but more selective towards the formation of the linear aldehyde compared to active species having triphenylphosphine ligands. The result was explained by the
15 presence of a network of water molecules bridging the sulfonato groups of the TPPTS ligands present in the active species (Fig. 15, b). The same species is stabilised through the formation of $\mathrm{SO}_{3}{ }^{-}$ - $\mathrm{Na}^{+}$ion pairs upon increasing of the ionic strength of the aqueous solution. ${ }^{185-187}$ The model ${ }^{180,184,188}$ based on these electrostatic 20 and/or ionic hydrogen bond interactions for the stabilisation of the bidentate ligand has been validated for other metal complexes and catalytic applications. ${ }^{179}$ Gulyás et al. found that hetero-complexes are generated almost exclusively as a result of attractive interactions between structurally simple ionic 


\section{Cite this: DOI: 10.1039/c0xx00000x}

\section{www.rsc.org/xxxxxx}

ARTICLE TYPE
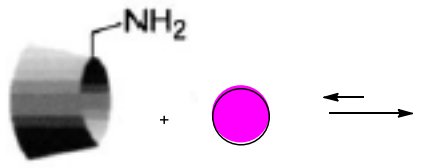

61

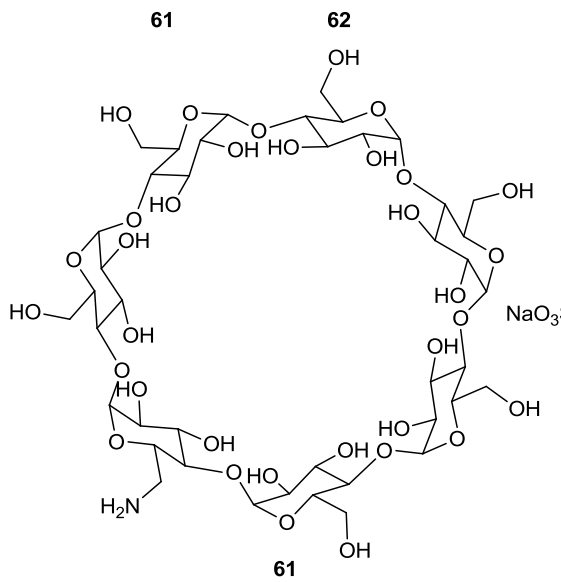

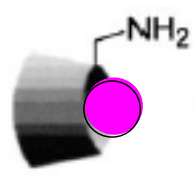

61-62<smiles>CC(C)(C)c1ccc(P(c2cccc(I)c2)c2cccc(OS(C)(=O)=O)c2)cc1</smiles>

b)

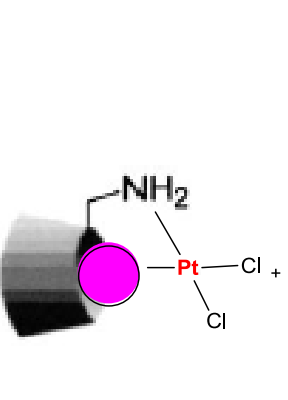

63
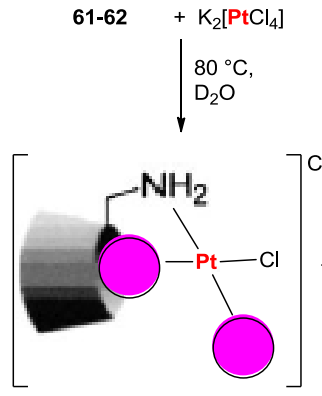

64

Fig. 17 Assembled supramolecular P,N ligands by means of hydrophobic interactions. (a) Schematic representation of the inclusion of $\mathbf{6 2}$ within $\mathbf{6 1}$.(b) After mixing 61-62 with $\mathrm{K}_{2}\left[\mathrm{PtCl}_{4}\right]$, three complexes are formed but only 63 is active for the hydrogenation of 2-methyl-3-buten-2-ol. Ref: see the text.

5 monophosphine ligands (see e.g. 56, Fig. 15, c). but so far no catalytic applications have been disclosed for the resulting metal complexes. ${ }^{189}$

The use of self-assembled ionic phosphine derivatives as ligands for catalysis has been disclosed by Pignatoro et al. These 10 authors evaluated the catalytic behavior of ligand combinations obtained by mixing in situ an acidic ligand (a chiral BINOLderived phosphite functionalized with a carboxylic acid group) and a basic ligand (a racemic BINOL-derived phosphite with a $\mathrm{NMe}_{2}$ group). ${ }^{190}$ The ligand heterocombinations of the amine- and acid15 derived phosphites displayed a slightly higher level of enantioselectivity than the corresponding ligand homocombinations in the rhodium-catalysed asymmetric hydrogenation of methyl 2-acetamidoacrylate $\mathbf{1 0}$ (up to $90 \%$ ee). NMR and IR studies failed to quantify the percentage of hetero20 complex formed for the best catalyst but a hetero-complex/homocomplexes ratio of 70/30 was assigned for a related catalytic system.

Hattori et al. recently reported the preparation of a supramolecular bidentate ligand by interlocking a crown ether 25 moiety, appended with a BINOL-derived phosphite ligand, with a phosphine-substituted ammonium derivative (57, Fig. 16, a) ${ }^{191}$ The high stability of the crown ether-ammonium salt interaction was used to link the two building blocks, each of which contributed one functional group to the final supramolecular hybrid bidentate

30 ligand with a pseudorotaxane structure. Rhodium complexes of this supramolecular ligand were better catalysts in asymmetric hydrogenation than those derived from the isolated building blocks of the pseudorotaxane (the wheel and the axle) or the heterocombination of the chiral phosphite and $\mathrm{PPh}_{3}$. A series of $\alpha$ 35 dehydroamino acid esters was hydrogenated with very high enantioselectivity (between 91 and $96 \%$ ee). ${ }^{191}$ At the same time, Fan and co-workers reported a very similar system, also based on pseudorotaxane skeleton (58, Fig. 16, a), that exhibits lower selectivity for the same catalytic reaction. ${ }^{192}$ The same group ${ }^{193}$ 40 pursued their investigation of the formation of bidentate ligands by means of ion-dipole interactions. ${ }^{194-196}$ They prepared the $\alpha, \omega$ bis(phosphite)polyether ligand 59 (Fig. 16, b) and studied the influence of alkali additives on the rhodium-catalysed hydrogenation of a series of $\alpha$-dehydroamino acid esters. The 45 effect is quite limited with a $17 \%$ enhancement of the ee in the more favourable case. Coordination chemistry studies surprisingly indicate that diphosphite ligand $\mathbf{5 9}$ alone acts as a chelate for the $\mathrm{Rh}$ metal centre. It explains the modest contribution of the cation additives on the catalytic reaction. An efficient allosteric catalyst 50 for rhodium-mediated asymmetric hydroformylation based on $\alpha, \omega$-bis(phosphite)polyether ligands was reported very recently by Vidal and co-workers. ${ }^{197}$ Diphosphite ligand 60 (Fig. 16, b) coordinates the $\mathrm{Rh}(\mathrm{acac}) \mathrm{CO}$ fragment as a bidentate ligand in the presence of $\mathrm{Cs}^{+}$and only as a monodentate ligand without this 55 additive. Again in the presence of $\mathrm{Cs}^{+}$, HP-IR analyses of the catalytic resting state in hydroformylation reactions reveal that the phosphorus atoms of $\mathbf{6 0}$ are coordinated to $\mathrm{Rh}$ in an equatorialequatorial fashion. 


\section{Cite this: DOI: 10.1039/c0xx00000x}

\section{www.rsc.org/xxxxxx}

ARTICLE TYPE

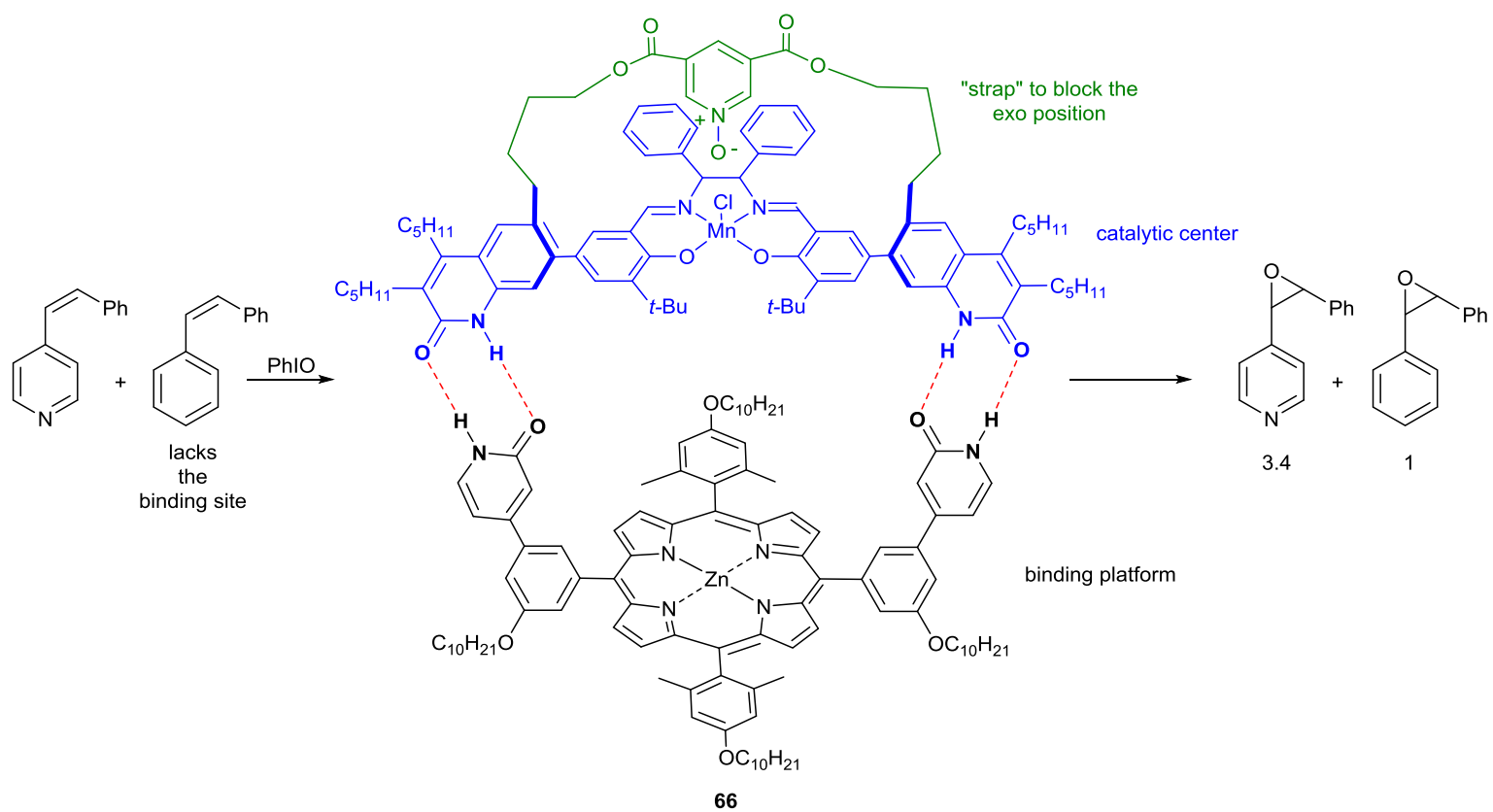

Fig. 18 Hydrogen-bonded dinuclear complex containing a receptor part (the Zn-porphyrin platform) for substrate binding and a catalytic part (the Mn(III)salen complex) for substrate activation. Second generation catalyst developed by Wärnmark and co-workers for substrate-selective epoxidation. Ref: see the text.

5 These observations explain the impressive effect of the $\mathrm{Cs}^{+}$ additive on the selectivity of the hydroformylation reaction (up to $62 \%$ enhancement of the ee for vinyl acetate).

Machut et al. demonstrated that hydrophobic interactions between $\beta$-cyclodextrin derivatives and water-soluble phosphines 10 enable the construction of self-assembled ligands in water. ${ }^{198} \mathrm{~A}$ $\beta$-cyclodextrin in which one of the primary hydroxyl groups has been replaced by an amino substituent was used as the host (61, Fig. 17, a). Inclusion of the hydrophobic part $\left(4-t-\mathrm{Bu}-\mathrm{C}_{6} \mathrm{H}_{4}\right)$ of the water-soluble monophosphine $\mathbf{6 2}$ within the cavity of $\mathbf{6 1}$ was 15 confirmed by a 2D T-ROESY NMR analysis performed in $\mathrm{D}_{2} \mathrm{O}$. The nitrogen atom of the host and the phosphorus atom of the guest are available for coordination to a metal centre. Upon addition of $\mathrm{K}_{2}\left[\mathrm{PtCl}_{4}\right]$ to the self-assembled ligand $\mathbf{6 1 - 6 2}$ in water, three complexes were formed which were identified as the neutral $(P, N)$ 20 chelate complex 63, the cationic $(P, N)$-chelate complex 64 and the monoligated $\left(\kappa^{1} P\right)$-complex 65 (see formulas in Fig. 17, b). Complex 63 was isolated and used as a precatalyst for the hydrogenation of 2-methyl-3-buten-2-ol in water at room temperature (initial TOF $=2600 \mathrm{~h}^{-1}$ ). This work represents the first 25 use of a designed bidentate supramolecular ligand for catalysis in water. Analogues of the $\beta$-cyclodextrin derivative 61, bearing more basic amino groups, favour the formation of neutral $\mathrm{Pt}$ and $\mathrm{Rh}$ complexes featuring the supramolecular P,N ligand in a chelate coordination mode. ${ }^{199}$ The related rhodium P,N-complexes are 30 selective catalysts for the hydroformylation of styrene in water (b:1 ratio $=10.1$ in the best case)..$^{200}$

Gennari and co-workers have used $\pi-\pi$ interactions between electron-rich and electron-poor arenes as the driving force to generate chiral hetero-complexes containing a combination of two 35 different monodentate phosphane ligands. In the highest performing combination in catalysis, BINOL-derived phosphites, with a pentafluorobenzyl or a methoxybenzyl group, were mixed in the presence of a rhodium precursor. A statistical distribution of rhodium complexes was observed, but the hetero-complexes were 40 more active and selective than the corresponding homo-complexes in the rhodium-mediated asymmetric hydrogenation of benchmark substrates. ${ }^{201}$ Schulz and co-workers prepared complementary mono(oxazolines) with appended substituents, one with an electron-rich (i.e. an anthracene group) and the other one with an 45 electron-poor (i.e. a 2,4,7-trinitrofluoren-9-one moiety) substituent. The aim was to generate supramolecular assemblies as a result of charge-transfer interactions. ${ }^{202}$ The resulting chiral catalysts were evaluated in the copper-catalysed Diels-Alder reactions of cyclopentadiene and acrylic acid derivatives. Some of 50 the reported homo- and heterocombinations of the mono(oxazoline) units exhibited a catalytic behaviour similar to standard bis(oxazoline) ligands in terms of selectivity and activity. However, the exact nature of the assembly interaction between the two mono(oxazoline) units was not established. The same group 55 reported the use of charge-transfer interactions to recover and reuse anthracene-modified bis(oxazoline) copper catalysts by precipitation in the presence of trinitrofluorenone. ${ }^{203,204}$ 


\section{Cite this: DOI: 10.1039/c0xx00000x}

\section{www.rsc.org/xxxxxx}

ARTICLE TYPE
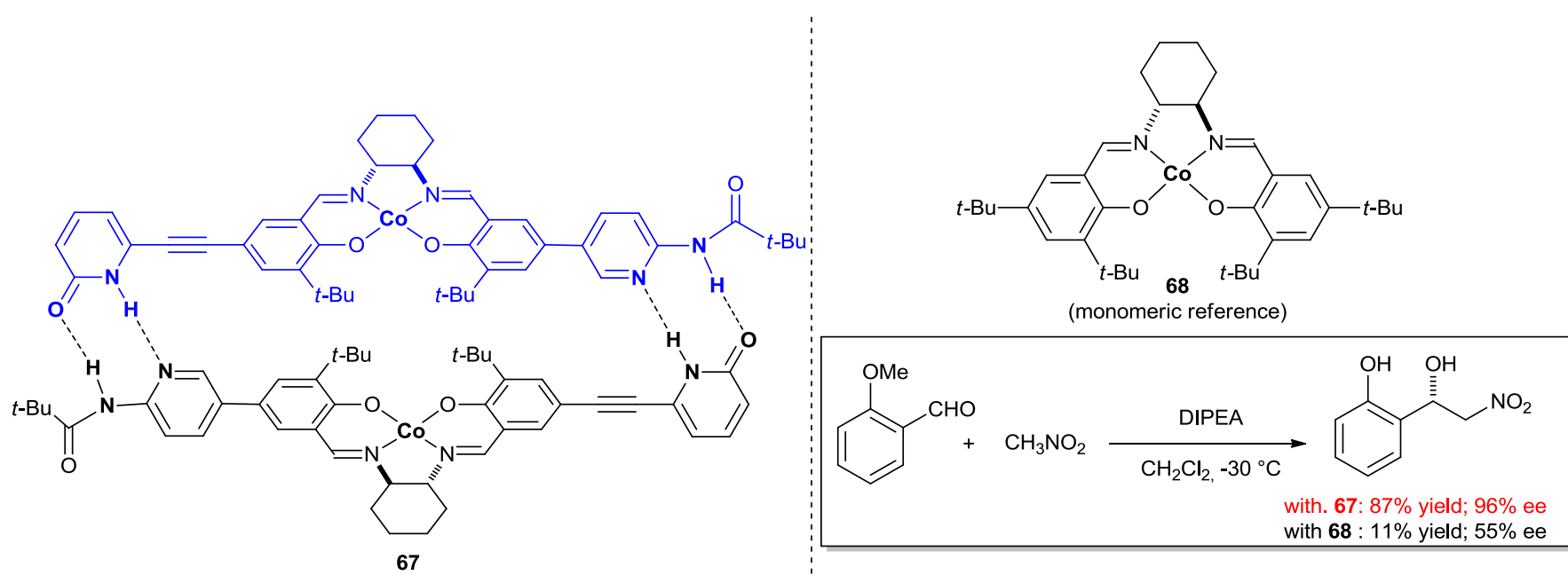

Fig. 19 Assembled head-to-tail dinuclear cobalt complex. Left: the dinuclear complex is formed as a result of complementary hydrogen bonds between the 2-pyridone and 2-amidopyridine groups. Right: Application in the asymmetric Henry reaction. DIPEA = N, $N$-Di-isopropylethylamine. Ref: see the text.

\subsection{Formation of di- and polynuclear complexes}

5 In all the examples mentioned above, supramolecular interactions have been limited to two ligands belonging to the same metal complex. The same strategies can be expanded to intermolecular ligand - ligand interaction generating di- or polynuclear complexes. Hydrogen bond interactions have been 10 used for the self-assembly of mononuclear complexes whereas other types of interactions mediate the formation of self-assembled photocatalysts.

\subsubsection{Assembled metal complexes through hydrogen bond} interactions

15 Wärnmark ${ }^{205-208}$ and Hong ${ }^{73,209,210}$ modified salen and porphyrin complexes by appending complementary hydrogen bond acceptor/donor pairs to the ligand scaffolds. Different applications were envisaged for the resulting hydrogen-bonded metal complexes: i) using one of the metal centres to bind and direct the 20 substrate to a second metallic catalytic site and, ii) promoting the cooperativity between two metal centres.

In their first example, Wärnmark and co-workers prepared a $\mathrm{Mn}(\mathrm{III})$-salen complex, an analogue of the Jacobsen-Katsuki catalysts for asymmetric alkenes epoxidation reactions, with two ${ }_{25}$ isoquinolinone groups appended to the ligand framework (catalytic part). ${ }^{205}$ The complementary sub-unit, which was designed as a platform for substrate binding (receptor part), was a Zn-tetraarylporphyrin with two 2-pyridone units at opposite mesoaryl porphyrin substituents. The resulting supramolecular 30 architecture was assembled through four hydrogen bonds established between the isoquinolinone and pyridone complementary groups. The resulting $\mathrm{Zn}, \mathrm{Mn}$-dinuclear complex was investigated as a substrate-selective catalyst for the epoxidation of a mixture of $c i$-alkenes substituted with a phenyl 35 group at one position and with a 4-pyridylalkyl or phenylalkyl substituents at the other. The underlying design principle in this investigation was that the alkene lacking a 4-pyridyl substituent as binding site for the $\mathrm{Zn}$-porphyrin unit, would react at a lower rate than the one containing the 4-pyridyl residue. However, at $20 \%$ 40 conversion, the selectivity is modest (the ratio of oxidation of pyridyl versus phenyl substrates is $1.55: 1$, at best). Further investigations on this system determined that the desired hetero-dinuclear complex, that combines the $\mathrm{Mn}$ and $\mathrm{Zn}$ platforms, constitutes, at most, $20 \%$ of the assemblies obtained by mixing the 45 catalytic and receptor parts. In addition, unselective reaction pathways (epoxidation outside of the cavity) compete with inner cavity reaction. ${ }^{206}$ The same group designed a second generation catalyst (66, Fig. 18), for which an alkyl strap containing a pyridine $\mathrm{N}$-oxide residue has been attached to the isoquinolinone groups of 50 the catalytic platform. The strapping had two objectives: i) forcing the isoquinolinone groups to adopt a cisoid conformation and, ii) blocking the outer face of the salen moiety (the unselective one). ${ }^{208}$ In this case, the selectivity increased to a 3.4:1 ratio in favour of the 4-pyridyl substituted substrate, probably, as a result, of a higher 55 concentration of the assembled hetero-dinuclear complex in solution and a reduced influence of the unselective pathway.

Intermolecular ligand - ligand assemblies were investigated in the $\mathrm{Cr}^{\mathrm{III}}$-catalysed asymmetric ring opening (ARO) of meso 60 epoxides with nitrogen nucleophiles. ${ }^{207}$ In this reaction, two molecules of catalyst are required for the activation of the substrate and the nucleophile. Compared to the covalent linking of two $\mathrm{Cr}^{\mathrm{III}}$ salen units, the supramolecular approach can lead to a more dynamic bimetallic catalyst as a better candidate for substrate 65 accommodation and transition state stabilisation. Various chiral $\mathrm{Cr}^{\mathrm{III}}$-salen mononuclear complexes were prepared which differ by the nature of the complementary group (isoquinolone or isoquinolinone) and the presence or not of an alkyl trap bridging the associating motifs. Compared to a mononuclear catalyst 70 lacking the complementary groups (taken as reference), both the homo-dinuclear and hetero-dinuclear 


\section{Cite this: DOI: $10.1039 / \mathrm{c0xx} 00000 x$}

\section{www.rsc.org/xxxxxx}

ARTICLE TYPE

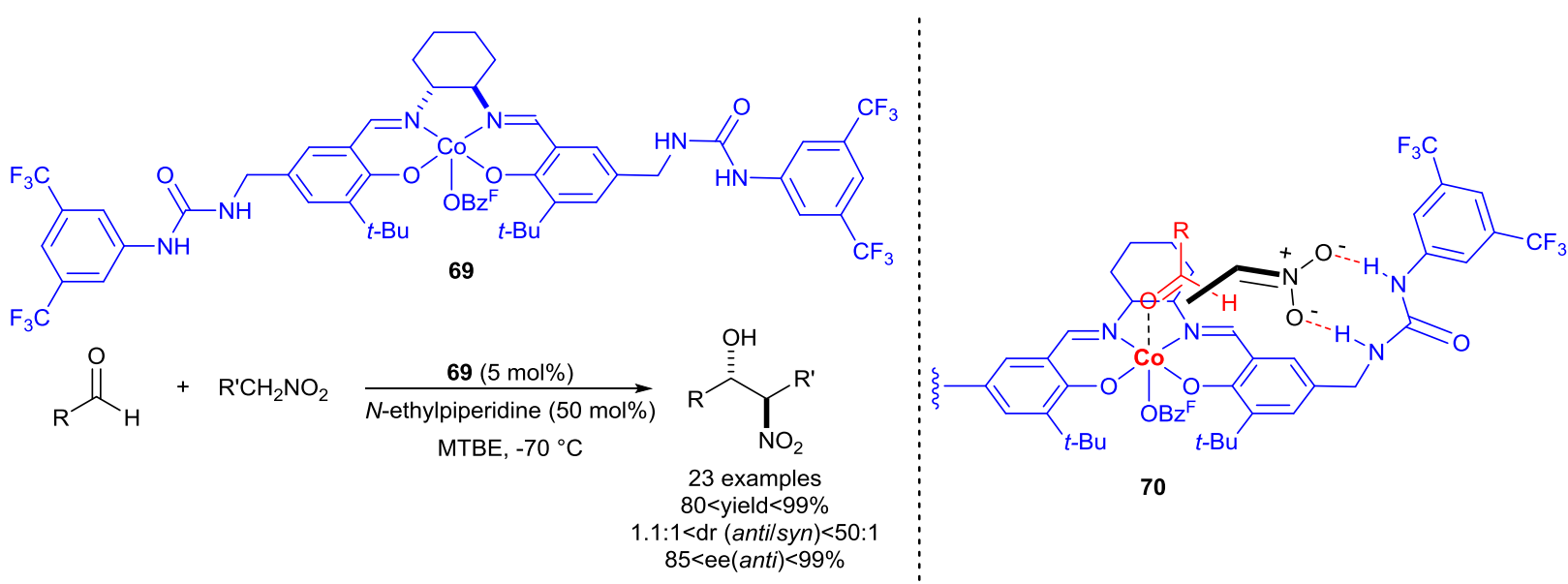

Fig. 20 Substrate-binding role played by the urea function in Co-catalysed Henry reactions. Left: selective formation of the anti-diastereomer in the asymmetric Henry reaction with Co-salen catalyst 69. Right: Proposed representation of the hydrogen-bonded ternary complex at the origin of the observed selectivity. $\mathrm{OBz}^{\mathrm{F}}=3,5$-bis(trifluoromethyl)benzoate. $\mathrm{dr}=$ diastereomeric ratio. Ref: see the text.

5 complexes, assembled through hydrogen bond interactions, exhibit larger reaction rate (up to 9 times) for the ring opening of cyclohexene oxide with $\mathrm{TMSN}_{3}$ but at the cost of lower enantioselectivities (ee $<10 \%)$.

Hong and co-workers attached a 2-pyridone unit at one side of 10 the Co-salen complex, and a complementary 2-amidopyridine moiety at the other, favouring the formation of a head-to-tail dinuclear complex (67, Fig. 19 left) as observed both in the solid state and in solution $\left(\mathrm{K}=53 \pm 21 \mathrm{~L} \cdot \mathrm{mol}^{-1}, \mathrm{CD}_{3} \mathrm{NO}_{2} 25 \% \mathrm{v} / \mathrm{v}\right.$ in $\left.\mathrm{CDCl}_{3}\right){ }^{209}$ This dinuclear catalyst was investigated in the Henry 15 reaction between aromatic aldehydes and nitromethane, where it outperforms both in terms of activity (48 times faster) and selectivity ( 96 vs $55 \%$ ee) the closely-related $\mathrm{Co}^{\mathrm{II}}$ mononuclear catalyst 68 (Fig. 19 right). Two units of Co-salen catalyst are involved in the transition state of the reaction (substrate and 20 reactant activation). Probably, the higher reaction rate measured for the hydrogen-bonded dinuclear complex is a consequence of the higher local concentration of the metal centres provided by the assembly.

The same hydrogen-bonded dinuclear complex proves to be less 25 efficient than the Jacobsen catalyst (a mononuclear complex) in the hydrolytic kinetic resolution (HKR) of epoxides. ${ }^{210}$ Hydrogen-bonding pairs between urea functions were envisaged as an alternative approach for the self-assembly of two mononuclear Co-salen catalysts. Bis-urea functionalized Co-salen complexes 30 were prepared which differ by the nature of the $N$-substituent of the urea functions located at their peripheries. The resulting Cosalen catalysts all showed significant rate acceleration (up to 13 times) for the HKR of of rac-epichlorohydrin when compared to the rate achieved with a catalyst lacking the urea groups (the 35 reference catalyst). Kinetic experiments revealed that the reaction is second order in cobalt whatever the nature of the cobalt catalyst (bis-urea Co-salen or reference catalyst). Compared to the reference, the best bis-urea Co-salen catalyst required shorter reaction times to resolve completely $(99 \%$ ee) epoxides at low 40 catalyst loading (0.03-0.05 mol\%). It also gave higher selectivity in the asymmetric hydrolysis of cyclohexane oxide (62\% yield, $75 \%$ ee compared to $9 \%$ yield, $45 \%$ ee for the reference). ${ }^{210} \mathrm{~A}$ control experiment with a Co-salen complex having $N$-methylated urea functions demonstrated that the urea $\mathrm{N}-\mathrm{H}$ groups are ${ }_{45}$ responsible for the observed rate acceleration. The self-association behaviour was probed by NMR analysis and the data can be fitted using a monomer-dimer model (dimerization constant $=56 \pm 22$ L. $\mathrm{mol}^{-1}$ in THF for a bis-urea Ni-salen complex structurally-related to the bis-urea Co-salen complex employed in catalysis). 50 Accordingly, the authors assumed that hydrogen bond interactions established through the urea functions induce ligand - ligand aggregation and this aggregation process is responsible for the observed activity and selectivity.

In striking contrast, the active species in asymmetric Henry ${ }_{55}$ reactions catalysed by the structurally-related $\mathrm{Co}^{\mathrm{III}}$-salen catalyst 69 is monomeric (Fig. 20 left). The role played by the urea functions in this reaction is markedly different than the one observed in the HKR of epoxides. One urea group in $\mathbf{6 9}$ acted as a binding site for the anionic nitronate nucleophile (70, Fig. 20). ${ }^{73}$

${ }_{60}$ This specific binding geometry for the ternary complex formed by the catalyst and the reactants led to an unusual selectivity for the reaction viz. formation of the anti diastereomer.

\subsubsection{Self-assembled photocatalysts}

Di- and oligotopic ligands and their respective di- and oligonuclear ${ }_{65}$ metal complexes have been applied in light-driven catalysis. ${ }^{211,212}$ Light-driven catalysis involves the combination of a metal catalyst and one or several photosensitizers in the same coordination complex. The idea at work is the activation of the catalytic centre through an energy and/or electron transfer process from the 70 photoactive residue. In the absence of the photoactive unit the catalyst is inactive. The photochemical reaction occurring 


\section{Cite this: DOI: 10.1039/c0xx00000x}

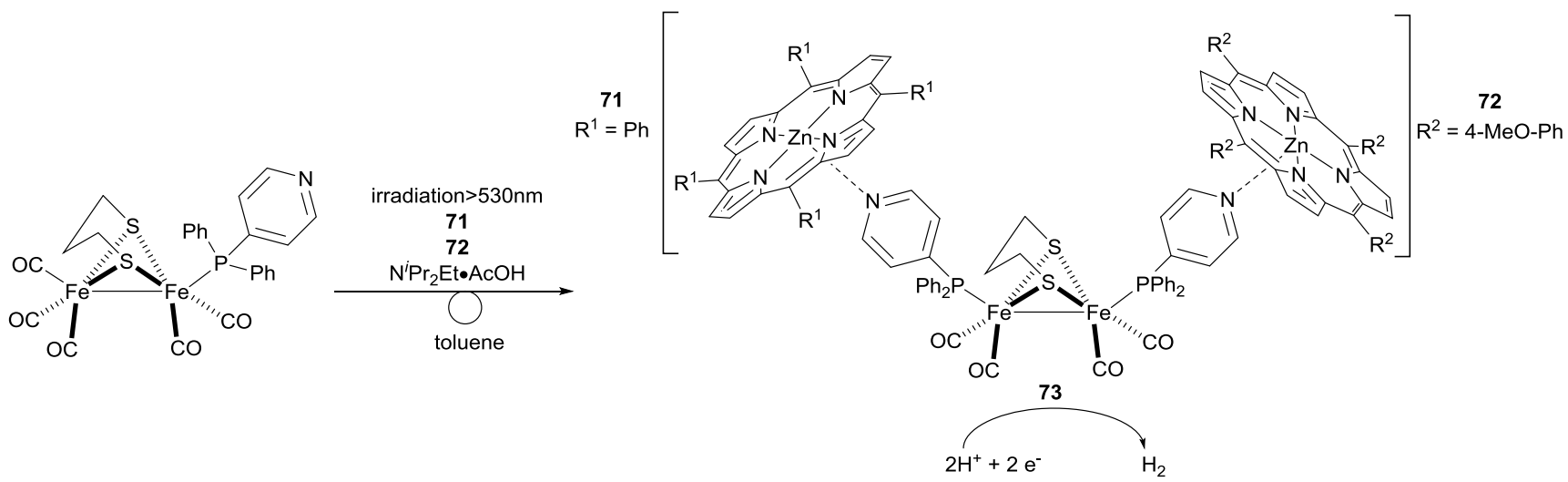

Fig. 21 Self-assembled photocatalyst for hydrogen generation. IR studies indicated that the active species is the self-assembled catalyst $\mathbf{7 3}$ which contains different chromophores (71 and 72). $\mathrm{N}^{i} \mathrm{Pr}_{2} \mathrm{Et} \cdot \mathrm{AcOH}$ acts as the proton source and sacrificial electron donor. Ref: see the text.
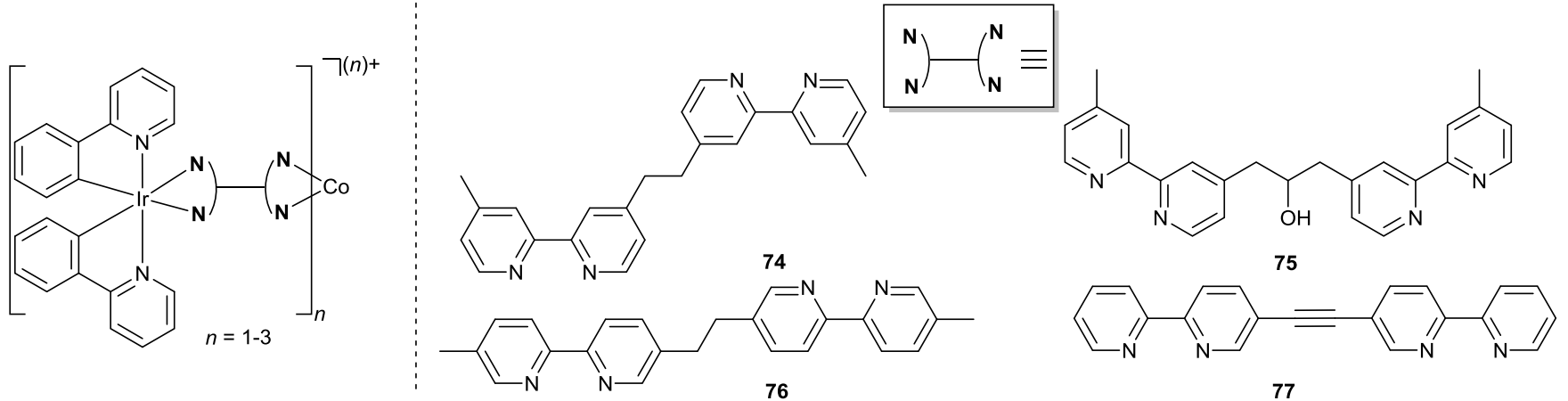

Fig. 22 Assembled Ir,Co oligonuclear complexes as photocatalysts for water reduction. In $\mathrm{H}_{2} \mathrm{O} / \mathrm{MeCN}$, and with TEOA as an electron donor, the selfassembled photocatalysts produced hydrogen upon irradiation. $p p y=2$-phenylpyridinate, $b p y=2,2$ '-bipyridine, TEOA $=$ triethanolamine. Ref: see the text.

through intramolecular sensitization is more effective than the 10 corresponding intermolecular version because charge-recombination and diffusion effects are significantly reduced. The photosensitizer and the metal catalyst are usually connected by means of a polydentate nitrogen ligand or a hybrid $\mathrm{P}, \mathrm{N}$ ligand. In many cases, the oligonuclear metal complexes used 15 in light-driven catalysis are prepared in a stepwise fashion ${ }^{213-218}$ and they are isolated before performing the catalytic experiment. ${ }^{219-226}$ This strategy prevents the direct screening of catalyst libraries (as described above for supramolecular bidentate ligand libraries used in homogenous catalysis). We will only 20 describe selected examples of self-assembled photocatalysts that can be easily tuned by changing one of their molecular building blocks.

Multi-components systems, incorporating a photosensitizer, a sacrificial electron donor and a transition-metal being an effective 25 water reduction catalyst (WRC), have been used in the photocatalytic generation of hydrogen. ${ }^{227}$ In supramolecular dyads, the WRC and the photosensitizer are connected through noncovalent interactions. ${ }^{212,228,229}$ Reek and co-workers ${ }^{230}$ designed an active site model of [2Fe2S] hydrogenases by replacing one 30 carbonyl ligand of a thiolate-bridged diiron hexacarbonyl complex by a pyridylphosphine ligand (Fig. 21). ${ }^{231}$ One coordination bond between the nitrogen atom of the pyridylphosphine and the metal atom of a zinc-porphyrin or zinc-salphen unit (the photosensitizer) enables the coupling of the WRC and the photosensitizer in the 35 same supramolecular structure. Several photosensitizer/diiron complex combinations have been investigated under visible light for the reduction of protons to hydrogen in the presence of $\mathrm{N}^{i} \mathrm{Pr}_{2} \mathrm{Et} \bullet \mathrm{AcOH}$ as the proton source and sacrificial electron donor. Surprisingly only a combination of the diiron complex and two 40 different zinc-porphyrin units was active $(30 \%$ yield, corresponding to $c a$. 2 turnovers per catalyst). The active species is likely the supramolecular aggregate $\mathbf{7 3}$ which contained the two different Zn-porphyrin units $\mathbf{7 1}$ and $\mathbf{7 2}$ (Fig. 21). Control experiments confirmed that two different bound porphyrin units 45 were required to obtain the active catalyst. Even though the reaction mechanism for hydrogen evolution is unclear, this work demonstrates that in situ association of the various building blocks constitutes an effective strategy to design photocatalysts. 
Jasimuddin et al. investigated several Ir-Co oligonuclear complexes as photocatalysts for the production of hydrogen from water. ${ }^{232}$ They prepared a series of cyclometallated Ir complexes (the photosensitizers) which bear one free bipyridine site available 5 for coordination to a Co centre. The Ir complexes differ by the nature of the linker between the Ir centre and the free bipyridine site. Self-assembly between these cyclometallated complexes $\left[\operatorname{Ir}(\text { ppy })_{2}(\mathrm{~L})\right]^{+}(\mathrm{L}=\mathbf{7 4 - 7 7}$, Fig. 22 $)$ and $\left[\mathrm{CoCl}_{2}\right]$ formed $\left[\mathrm{Co}(\mathrm{bpy})_{n}\right]^{2+}$-type complexes $(\mathrm{n}=1-3$, ppy $=2$ - phenylpyridinate, 10 bpy $=2,2^{\prime}$-bipyridine). Upon irradiation, the self-assembled complexes produced $\mathrm{H}_{2}$ from water in presence of TEOA as the electron donor. As expected, the nature of the linker had a large impact on the efficiency of the self-assembled photocatalyst. The catalyst derived from $\mathbf{7 4}$ is the only one that is more active than the 15 model system, viz. the non-assembled catalyst composed of $\left[\operatorname{Ir}(\mathrm{ppy})(\mathrm{bpy})_{2}\right]^{+}$and $\left[\mathrm{Co}(\mathrm{bpy})_{3}\right]^{2+}$. Although spectroscopic analyses support an intramolecular quenching of the excited state by the $\mathrm{Co}^{\mathrm{II}}$ centre, other quenching pathways are feasible that affect the overall efficiency of the self-assembled photocatalysts.

20 Feiters and co-workers attempted to extend the nature of the non-covalent interactions that can be used to design self-assembled photocatalysts. ${ }^{233,234}$ They studied a supramolecular version of the three-component heterogeneous photocatalytic systems developed independently by Lehn ${ }^{235}$ and Grätzel ${ }^{236,237}$ more than 30 years 25 ago. Here, the system was composed of: i) an iridium sensitizer appended with a $\beta-C D$ unit, ii) a viologen derivative bearing two adamantyl units (the electron relay), iii) Pt nanoparticles stabilised with a perthiolated $\beta$-cyclodextrin (Pt- $\beta$-CD, the WRC), and (iv) EDTA as the sacrificial electron donor. The iridium, viologen and $30 \mathrm{Pt}$ building blocks can potentially associate through hydrophobic interaction between the $\beta$-CD units borne by the sensitizer and the Pt nanoparticles and the two adamantyl groups of the viologen derivative. Compared to Lehn and Grätzel systems, this strategy presents the following advantages: i) the electron transfer can be 35 better controlled and, ii) a variety of functional photoactive systems can be generated from a limited number of building blocks. The efficiency of the self-assembled photocatalyst was evaluated in the reduction of water into $\mathrm{H}_{2}$ upon irradiation. However, the catalytic self-assembly proved to be less efficient 40 than the system of reference using a methyl-substituted viologen, an electron relay that do not form inclusion complexes with the $\beta$ CD units. This surprising result can be explained by the higher tendency of the adamantyl-modified viologen, compared to the methyl-substituted one, to aggregate into electro-inactive ${ }_{45}$ species. ${ }^{238}$ Absence of complementary groups between the various building blocks also lead to a reduced amount of the desired complex in solution, i.e. the Ir sensitizer - viologen - Pt- $\beta$-CD supramolecular assembly. Further work is needed in order to improve the catalytic efficiency of the system towards hydrogen 50 production: a possible direction is the design of a viologen derivative equipped with two different hydrophobic substituents as possible recognition motifs of the photosensitizer and the $\mathrm{Pt}$ nanoparticles respectively.

In this section, we have compiled catalytic applications of ${ }_{55}$ supramolecular bidentate ligands and supramolecular polynuclear complexes. We will discuss the scope and limitations of these systems and give some prospective perspectives for this strategy in the last section of this review.

\section{Ligand - ligand additive interaction}

\section{${ }_{60} 4.1$ Modification of monodentate ligands}

Tailoring the activity of a given catalyst by modifying its first coordination sphere is a basic concept of homogeneous catalysis. Cone angle $(\theta),{ }^{239} \chi$-parameter ${ }^{240}$ and tilt angle ${ }^{241-244}$ are important descriptors of the steric and electronic properties of phosphine ${ }_{65}$ ligands. However, despite important progress in ligand design by means of these parameters and computational techniques, the activity and selectivity of a catalyst remains difficult to predict. This is even more true in asymmetric catalysis where "achieving $95 \%$ ee" involves energy differences of about $2 \mathrm{kcal} .{ }^{245} \mathrm{Up}$ to now, 70 the development of highly efficient catalysts requires an endeavour of ligand synthesis, whose efficiency is enhanced by a trial-anderror iteration. Monodentate ligands gained increasing attention during the last years due to their relative simplicity, accessibility and their successful uses in various catalytic reactions. BINOL75 based phosphites, ${ }^{246}$ phosphoramidites ${ }^{247}$ and phosphonites ${ }^{248}$ are powerful ligands for asymmetric hydrogenation. Reetz demonstrated that the use of mixtures of chiral monophosphines, also in combination with achiral monophosphines, is a powerful tool in the discovery of efficient catalysts. ${ }^{137,249-251}$ A modular 80 approach, for example using sugar ${ }^{252,253}$ or peptide 254,255 backbones, reduces the synthetic effort but the preparation of a new ligand still requires covalent synthesis.

An alternative approach consists in modifying the properties of a simple monodentate phosphine by means of attractive non85 covalent interactions between this ligand and a well-chosen complementary additive. The additive alters both the first and second coordination sphere of a given transition-metal and as a result strikingly modifies its catalytic properties.

The proof-of-concept for this strategy was demonstrated by van ${ }_{90}$ Leeuwen and Reek in 2001. They used the zinc-porphyrin platform 71, already mentioned above for the building of supramolecular photocatalysts (Fig. 21), to modify the electronic and steric properties of a series of pyridylphosphine ligands (ligand - template strategy). ${ }^{57}$ The pyridylphosphine ligands employed in 95 this seminal study differ by the nature (para-pyridyl or metapyridyl groups) and number of pyridyl groups linked to the phosphorus atom (40, 78-80, Fig. 23). The success of the ligandtemplate strategy is dictated by the directionality and the strength $\left(10^{3}<\mathrm{K}<10^{4} \mathrm{~L}^{\mathrm{m}} \mathrm{mol}^{-1}\right)$ of the coordination bond established 100 between the pyridyl group of the pyridylphosphine ligand and the zinc atom of the zinc-porphyrin platform. Coordination chemistry studies of the pyridylphosphine ligands 40, 78-80 with $\left[\mathrm{PdCl}_{2}(\mathrm{cod})\right]$ and $\left[\mathrm{Rh}(\mathrm{acac})(\mathrm{CO})_{2}\right]$ precursors yielded the expected complexes $\left[\mathrm{PdCl}_{2} \mathrm{~L}_{2}\right]$ and $\left[\mathrm{Rh}(\mathrm{acac}) \mathrm{L}_{2}(\mathrm{CO})\right]$ respectively (where $\mathrm{L}$ $105=40,78,79$ or 80). Upon addition of two equiv. of 71, ligand 78 binds the zinc-porphyrin platform selectively through its $\mathrm{N}$ atom and this binding does not have a significant impact on its $\mathrm{P}$ atom because bis-ligated $\left[\mathrm{PdCl}_{2}(\mathbf{7 8} \cdot \mathbf{7 1})_{2}\right]$ and $\left[\mathrm{Rh}(\mathrm{acac})(\mathbf{7 8} \cdot \mathbf{7 1})_{2}(\mathrm{CO})\right]$ complexes are obtained. In striking contrast, the coordination of 110 three zinc- 


\section{Cite this: DOI: 10.1039/c0xx00000x}

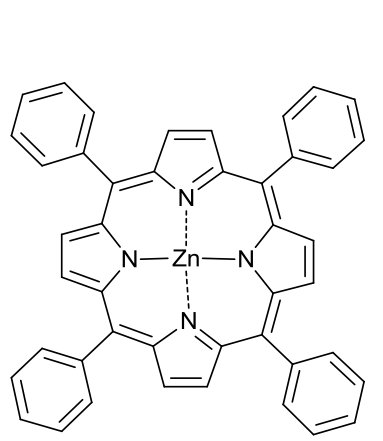

71<smiles>c1ccc(P(c2ccccc2)c2ccncc2)cc1</smiles><smiles>c1ccc(P(c2cccnc2)c2cccnc2)cc1</smiles>

79<smiles>c1ccc(P(c2ccccc2)c2cccnc2)cc1</smiles><smiles>c1cncc(P(c2cccnc2)c2cccnc2)c1</smiles><smiles>c1cc(P(c2ccncc2)c2ccncc2)ccn1</smiles>

81<smiles>CC(C)(C)c1cccc2c1O[Te]1(Oc3c(C(C)(C)C)cccc3C(C)(C)C)Oc3cc(Cl)c(Cl)cc3N=C2n2cc1c1ccccc12</smiles>

80

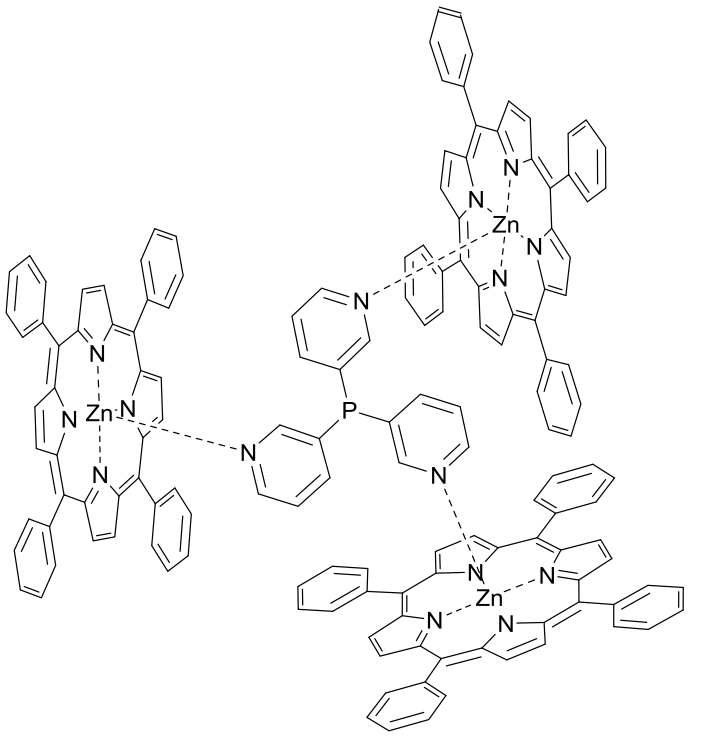

$80 \cdot 71_{3}$

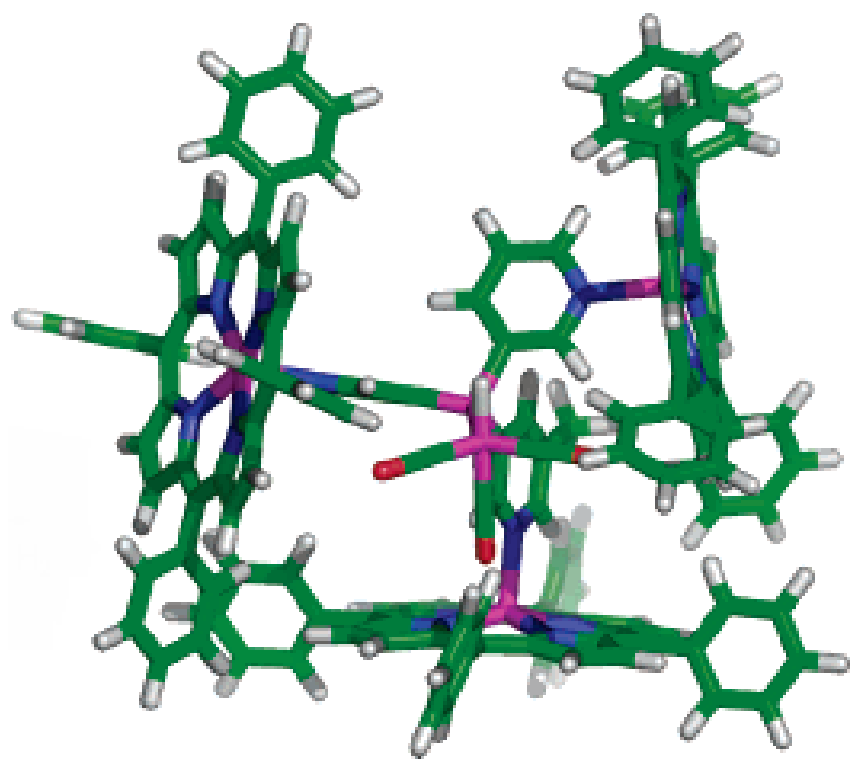

Fig. 23 Ligand - template strategy. Above: zinc-porphyrin 71 and zinc-salphen 82 platforms used to modify the coordination properties of the pyridylphosphine ligands 40, 78-81. Below: Self-assembled ligand $\mathbf{8 0} \bullet \mathbf{7 1}_{3}$ and molecular model of the catalytic resting state $\left[\mathrm{Rh}(\mathrm{H})(\mathbf{8 0} \bullet \mathbf{7 1} 3)(\mathrm{CO})_{3}\right]$ in 5 hydroformylation reactions. (The molecular model is reprinted with permission from ref. 258. Copyright 2006. American Chemical Society). Refs: see the text.

porphyrin units to the pyridyl groups of $\mathbf{8 0}$ impacts the coordination number of the metal. Thus, the addition of six equiv. of 71 to $\left[\mathrm{PdCl}_{2}(\mathbf{8 0})_{2}\right]$ and $\left[\mathrm{Rh}(\mathrm{acac})(\mathbf{8 0})_{2}(\mathrm{CO})\right]$ yielded the 10 monoligated complexes $\left[\mathrm{PdCl}_{2}(\mathbf{8 0} \cdot \mathbf{7 1})\right]$ and $\left[\mathrm{Rh}(\mathrm{acac})\left(\mathbf{8 0} \cdot \mathbf{7 1}_{3}\right)(\mathrm{CO})\right]$ respectively with the concomitant dissociation of one ligand - template assembly $\mathbf{8 0 \cdot 7 1 3}$ (enforced ligand dissociation). A later study reveals that the stepwise complexation process of $\mathbf{7 1}$ with $\mathbf{8 0}$ shows signs of positive 15 cooperativity, i.e. the third $\mathbf{7 1}$ unit binds more strongly than the first two ones as a probable result of favourable $\pi$ - $\pi$ interactions with the meso-phenyl groups of the two porphyrins already coordinated to $\mathbf{8 0}$. $^{256}$

Here, the impact of the supramolecular interactions is evaluated 20 by comparing the catalytic properties in hydroformylation reactions of linear aldehydes, of the assembled ligands $\mathbf{7 8} \cdot 71$,

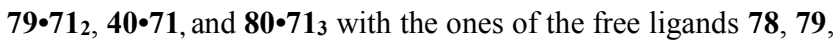
40 and 80. No striking differences are observed between the catalytic behaviour of the assembled and free ligands $\mathbf{7 8}$ and $\mathbf{4 0}$. 25 However, 79-712 led to a 2 -fold increase in activity and a different selectivity in the rhodium-catalysed hydroformylation of 1-octene. As expected, the effect is even larger for $\mathbf{8 0 \cdot 7 1 3}$; the respective rhodium catalyst is 10 times more active and the branched aldehyde is formed preferentially $(1: \mathrm{b}$ ratio $=0.6)$. The replacement 30 of the zinc-porphyrin platform $\mathbf{7 1}$ by a less dynamic $\mathrm{Ru}^{\mathrm{II}}$-porphyrin led to an even more selective catalyst $(1: b$ ratio $=0.4) .{ }^{256}$ The higher activity can be explained by the fact than monoligated rhodium catalysts are more active than the respective bis-ligated catalysts, but the 


\section{Cite this: DOI: 10.1039/c0xx00000x}

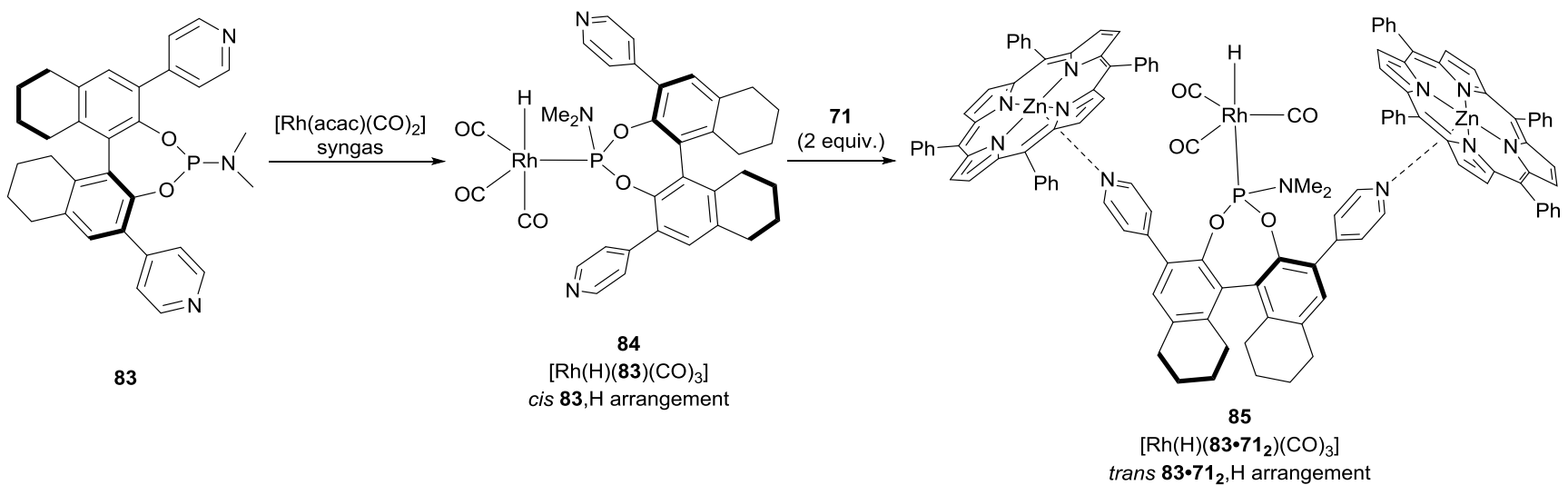

Fig. 24 Ligand - template assembly of two Zn-porphyrin templates with a monodentate phosphoramidite ligand. Coordination modes of ligand $\mathbf{8 3}$ alone

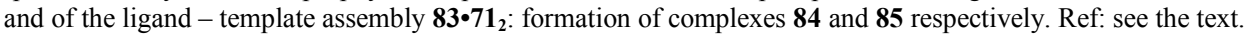

observed selectivity is unusual for a catalyst bearing a hindered 5 monodentate ligand. ${ }^{257}$ Most likely, the high selectivity derives from the encapsulation of the rhodium catalytic centre by the surrounding ligand - template assembly (see the molecular model of the catalytic resting state $\left[\mathrm{Rh}(\mathrm{H})(\mathbf{8 0 \cdot 7 1 3})(\mathrm{CO})_{3}\right]$, Fig. 23). Indeed, steric repulsions imposed on the metal-substrate complex 10 inside the capsular assembly are thought to play an important role towards the observed selectivity. This was confirmed by the fact that the catalyst derived from $\mathbf{8 0 . 7 1 3}$ is able to discriminate between carbon atoms $\mathrm{C} 3$ and $\mathrm{C} 4$ in trans-3-octene providing the innermost aldehyde in $75 \%$ yield. ${ }^{258}$ This regioselectivity is likely 15 determined during the hydride migration step where the rotational freedom of the substrate is reduced by the steric restrictions imposed inside the capsule. Interestingly, Reek and co-workers recently found that the rhodium catalyst containing the ligand template assembly $\mathbf{8 0 \cdot \mathbf { 7 1 } _ { 3 }}$ is able to maintain its unusual selectivity 20 towards the formation of branched aldehydes at $80{ }^{\circ} \mathrm{C}$, a relevant temperature for a potential industrial application. ${ }^{259}$ High pressure of CO (107 bar) and an excess of 71 (6 equiv. with respect to 80) are required to prevent the formation of unselective bis-phosphine rhodium species and the dissociation of the Zn-porphyrin from the ${ }_{25}$ pyridylphosphine ligand that occurs under these relatively harsh conditions.

Further investigation of this system with $\mathbf{8 1}$, the para-pyridyl analogue of $\mathbf{8 0}$, highlights its originality. The coordination of three equivalents of the zinc-porphyrin unit $\mathbf{7 1}$ or of the zinc-salphen 30 unit $\mathbf{8 2}$ to the nitrogen atoms of $\mathbf{8 1}$ yielded the respective ligand template assemblies $\mathbf{8 1 \cdot 7 1 _ { 3 }}$ and $\mathbf{8 1 \cdot 8 2 _ { 3 }}$ as confirmed by UV-vis titrations. ${ }^{260}$ While X-ray analysis confirmed the expected

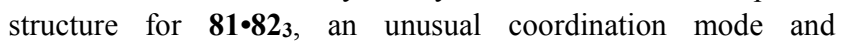
stoichiometry is observed for $\mathbf{7 1 :} \mathbf{8 1}_{2} \mathbf{\bullet 7 1}_{5}{ }^{261}$ In the solid state, a 35 hexa-coordinate zinc ${ }^{\mathrm{II}}$ complex is observed which is bound to one pyridyl group of two $\mathbf{8 1 \cdot 7 1 _ { 3 }}$ units. This coordination behaviour probably results from favourable $\pi-\pi$ interactions present in the crystal packing. Ligand - template assemblies $\mathbf{8 1 \cdot 7 1 3}$ and $\mathbf{8 1 \bullet 8 2 3}$ display phosphine atoms less well encapsulated and thus more 40 accessible for metal coordination than that of $\mathbf{8 0 \cdot 7 1 3}$. As a result, the catalysts derived from $\mathbf{8 1}$ are less active and present a classical selectivity towards the formation of the linear aldehyde in the hydroformylation of 1-octene. ${ }^{261}$ Various zinc-porphyrin platforms, as well as pyridylphosphines and pyridylphosphites, 45 were evaluated demonstrating the wide range of catalysts that can be built by the ligand - template strategy. With ligand $\mathbf{8 0}$, assemblies with zinc-salphen ${ }^{260}$ or zincbis(thiosemicarbazonato ${ }^{262}$ platforms gave slightly less efficient catalysts than the ones based on porphyrin scaffolds. First attempts 50 to get asymmetric version of these catalysts were not successful either using a chiral salen building block or chiral pyridylphosphite ligands.

Recently, Bellini et al. described an ingenious application of the ligand - template strategy (Fig. 24). ${ }^{263}$ They designed the chiral ${ }_{55} \mathrm{H}_{8}$-BINOL-derived phosphoramidite ligand $\mathbf{8 3}$ which contain two 4-pyridine rings located at the 2,2'-positions of the $\mathrm{H}_{8}$-BINOL backbone. In contrast to the binding behaviour of the Zn-porphyrin platform 71 with 80, the stepwise association of $\mathbf{7 1}$ with $\mathbf{8 3}$ is not cooperative, i.e. that the association constants for the first and ${ }_{60}$ second porphyrin binding are similar ( ca. $\left.2 \times 10^{3} \mathrm{~L}_{\mathrm{mol}}{ }^{-1}\right)$. The nature of the catalytic resting state in hydroformylation reactions was determined by HP-NMR studies. The reaction between $\mathbf{8 3}$ and $\left[\mathrm{Rh}(\mathrm{acac})(\mathrm{CO})_{2}\right]$ under syngas pressure yielded a monoligated complex $\left[\mathrm{Rh}(\mathrm{H})(\mathbf{8 3})(\mathrm{CO})_{3}\right]$ as expected for a bulky 65 monophosphite ligand. The coordination number of the $\mathrm{Rh}$ atom is not affected by the presence of two equivalents of 71 as the

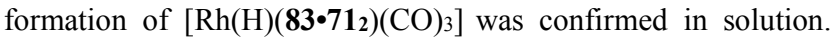
More surprisingly, NMR data are consistent with the ligand template assembly $\mathbf{8 3 \cdot 7 1 _ { 2 }}$ located in the trans position of the 70 hydride ligand (complex $\mathbf{8 5}$ ) whereas ligand $\mathbf{8 3}$ alone assumed the classically-observed cis arrangement (complex 84). ${ }^{263}$ This unusual coordination mode for the ligand - template assembly is reflected in catalysis: compared to $\mathbf{8 4}$, catalyst $\mathbf{8 5}$ is more active when applied in the hydroformylation of trans-2-octene. It also 75 exhibits a different selectivity: the innermost aldehyde (2ethylheptanal) is formed with a higher degree of optical purity. More precisely, $56 \%$ conversion, a ratio 


\section{Cite this: DOI: $10.1039 / \mathrm{c0xx00000x}$}

(a)<smiles>[R]C(OC(C)(C)C)[N+](=O)[O-]</smiles><smiles>[R12]C=C1c2cc[R14]cc2C1[R1]</smiles>

(c)

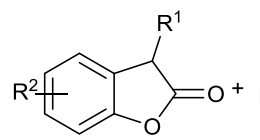

$$
\mathrm{R}^{3}=\mathrm{Ph} \text { or } \mathrm{Ar}
$$<smiles>[R]C=CCC([R])(CC=C[R])[N+](=O)OC(C)(C)C</smiles>

11 examples $90<$ yield $<99 \%$ $90<$ ee $<97 \%$<smiles>COc1c(-c2ccc3ccccc3c2)cc2ccccc2c1-c1cc2ccccc2c(OC)c1-c1ccc2ccccc2c1</smiles>

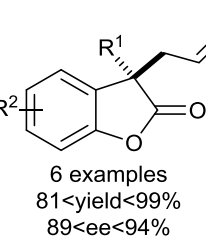

$\sim \mathrm{CO}_{2} t-\mathrm{Bu}$

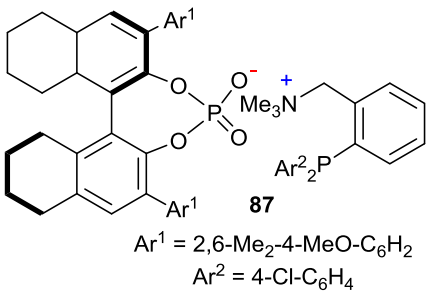

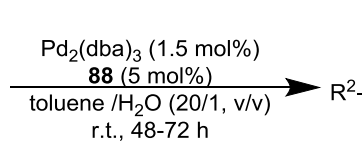

r.t., $48-72 \mathrm{~h}$

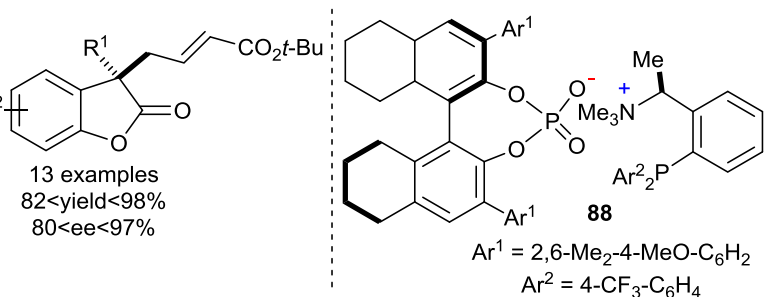

Fig. 25 Chirality transfer between an achiral monodentate ligand and a chiral module by means of electrostatic interactions. (a) Ion-pairing ligand $\mathbf{8 6}$ is efficient for the asymmetric allylation of $\alpha$-nitrocarboxylates with cinnamyl carbonate derivatives. Ion-pairing ligands $\mathbf{8 7}$ and $\mathbf{8 8}$, with a chiral phosphate anion (instead of a phenolate ion in case of $\mathbf{8 6}$ ), catalyses the allylation of benzofuran-2(3H)-ones. (b) 87 is the best self-assembled ligand for the 5 allylation of benzofuran-2(3H)-ones with ester-functionalized allylic carbonate. (c) $\mathbf{8 8}$ is the best ligand for the same reaction involving simple allylic carbonate derivatives. Refs: see the text.

of 2-methyloctanal:2-ethylheptanal equals to 0.96 and $45 \%$ ee for 2-ethylheptanal is obtained with catalyst $\mathbf{8 5}$ to be compared with $12 \%$ conversion, a ratio of 1.41 and $25 \%$ ee with catalyst 84 . ${ }_{10}$ Control experiments confirmed that the binding of the zincporphyrin unit is required to increase the catalyst efficiency. A small series of Lewis acidic platforms ( $\mathrm{Zn}$ - or boron-based) was investigated for the hydroformylation of several internal alkenes and the same trend was observed. A more precise investigation of 15 the system $^{264}$ indicated that the size of the platform neither influenced the coordination mode nor the catalytic performance. Indeed, the ligand - template assembly is always located trans to the hydride ligand whatever the binding partner ( $\mathrm{Zn}$ - or boronbased) of the pyridylphosphane ligand and trans complexes always 20 give higher selectivity and activity than the cis ones. Electronic effects induced by the template on the phosphorus atom are most likely responsible of the difference in selectivity observed between trans and cis isomers.

The modification of monodentate ligands by means of the ligand 25 - template strategy proved to be an efficient tool for tuning the coordination spheres of various metal catalysts. The concept is not restricted to the use pyridylphosphine ligands as pyridylNHC, ${ }^{265,266}$ pyridyl-salen ${ }^{267}$ and pyridyl-imine ${ }^{268}$ ligands (Fig. 28) were also prepared and the effect of their supramolecular 30 anchoring to Lewis acidic platforms was investigated in catalysis.

In order to expand the scope of the catalytic reactions that can benefit from the interaction between a ligand an additive, several catalytic systems have been designed recently. Hydrogen bonding, electrostatic and hydrophobic interactions have been used to 35 assemble the complementary groups of monodentate ligands and additives.

Clarke prepared a chiral phosphine tagged with an amidonapthyridine moiety and studied the influence of achiral pyridone additives in catalysis. ${ }^{269}$ Amidonapthyridine and 40 pyridone are well-known complementary groups and the well-defined hydrogen bond interactions were thought to generate an assembled ligand with different catalytic properties compared to the monodentate ligand. Positive effects were observed both in the asymmetric hydrogenation of a cyclic imine and the 45 hydrosilylation of 4-fluoroacetophenone, although the benefits were quite modest compared to the successful examples mentioned above for the ligand - template strategy.

Ooi and co-workers elegantly demonstrated that very efficient chirality transfer occurs between an achiral monodentate ligand 50 and a chiral module assembled by means of electrostatic 
a)

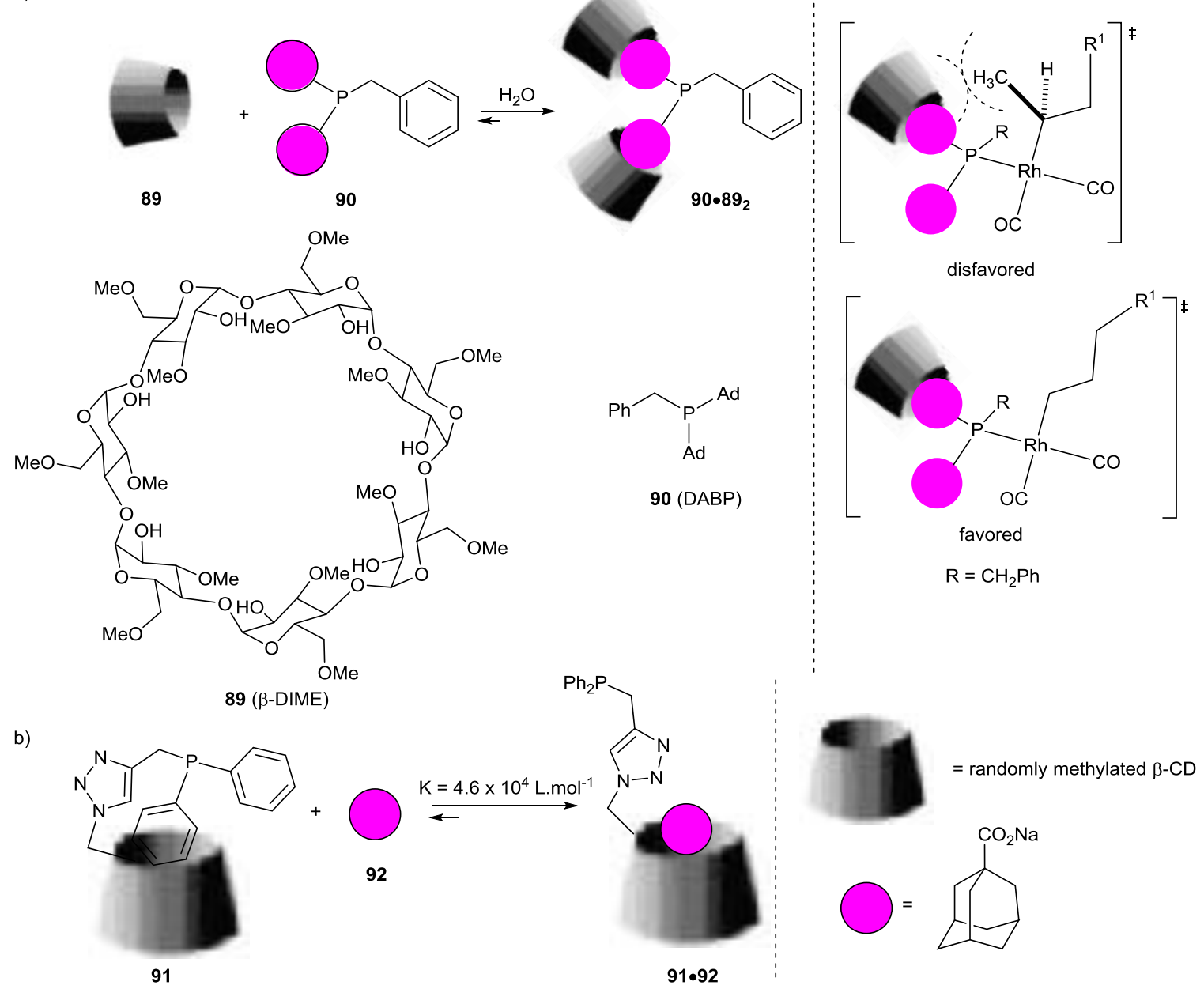

Fig 26 Hydrophobic interactions as a tool to modify the coordination spheres of metal phosphine complexes. (a) Hydrophobic interactions between one equivalent of $\beta$-DIME and one adamantyl group of the DABP ligand improve the selectivity of the respective catalyst towards the formation of the linear aldehydes. Schematic representation of the Rh-alkyl intermediates in the hydroformylation reaction of linear olefins. (b) The equilibrium between 91 and

592 is strongly shifted towards the formation of the inclusion complex $\mathbf{9 1 . 9 2}$. Catalyst derived from $\mathbf{9 1 . 9 2}$ is more selective than 91 in the rhodiumcatalysed hydroformylation reaction of methyl-4-pentenoate $(1: \mathrm{b}$ ratio $=1.78$ for $\mathbf{9 1 \bullet 9 2 , ~} 1: \mathrm{b}$ ratio $=0.67$ for $\mathbf{9 1})$. $\mathrm{DABP}=\operatorname{di}(1$-adamantyl $)$ benzylphosphine. Refs: see the text.

interactions. ${ }^{270}$ They prepared ion-pairing ligands composed of an achiral cationic ammonium-phosphine hybrid ligand and a chiral 10 binaphtholate monoanion arranged in close proximity to one another. These self-assembled ligands were tested in combination with $\mathrm{Pd}_{2}(\mathrm{dba})_{3}$ for the asymmetric allylation of $\alpha$ nitrocarboxylates with cinnamyl carbonate derivatives (Fig. 25, a). Control experiments indicated that: i) the formation of an ion pair 15 between the two components is required to get enantioselectivity, and ii) the spatial arrangement of the various building blocks dictated the catalytic efficiency; ortho-diphenylphosphinobenzylammonium being the more suitable cation tested. Ion-pairing ligand $\mathbf{8 6}$ proved to be particularly efficient providing up to $97 \%$ 20 ee. Kinetic experiments and the observation of a positive nonlinear effect in the palladium-catalysed allylation reaction allow the authors to propose a mechanism. The reactive species in the bondforming event is probably a $\mathrm{Pd}^{\mathrm{II}}$ allyl complex bearing two ionpairing ligands 86. The alcohol group of the binaphtholate anion ${ }_{25}$ (see the hydrogen in bold in $\mathbf{8 6}$, Fig. 25, a) played a crucial role during the catalysis. It is involved in a hydrogen bond interaction with the naphtholate anion (observed in the solid state) and is thought to interact with the nucleophilic nitronate anion during the catalytic cycle.

30 The same system must be slightly adapted to efficiently catalyse the asymmetric allylation of benzofuran-2(3H)-ones. ${ }^{271}$ Chiral $\mathrm{H}_{8}-$ BINOL-derived phosphates are more suitable anions in that case. After a screening of a series of ion-pairing ligands, $\mathbf{8 7}$ and $\mathbf{8 8}$ proved to be the more efficient ligands for the allylation of 35 benzofuran-2 $(3 H)$-ones with functionalized allylic carbonates and 


\section{Cite this: DOI: 10.1039/c0xx00000x}

\section{www.rsc.org/xxxxxx}

simple allylic carbonates respectively (Fig. 25, b and c). Chirality is present on both the cationic and anionic components of ligand 88. The matched chiral cation - anion combination, $(R)$-phosphate anion and $(S)$-1-(2-phosphinophenyl)ethylammonium cation, is 5 more selective than its diastereoisomer (the mismatched combination). On the one hand, a limitation of the system is that the ion-pairing ligands have to be isolated before performing catalysis. On the other hand, this work demonstrates that high level of stereocontrol can be achieved by means of metal catalysts which 10 combine well-defined secondary interactions and remote chiral centre(s). Chiral anions derived from the BINOL backbone are now ubiquitous in supramolecular catalysis; they are used as counteranions for cationic metal complexes and for cationic intermediates in organocatalysis (see sections 5 and 6). .,53,63,272

15 Native or modified cyclodextrins (CDs) have found many applications in catalysis performed in water depending on the reaction partners encapsulated in their hydrophobic pocket. ${ }^{200,273}$ They can act as: i) mass transfer promoters in case of substrates that are poorly soluble in water (CD - substrate interaction), ${ }^{200,273}$

20 ii) reaction flasks by binding a substrate within their cavity and orientating it towards a catalytic centre located at their upper part (CD - substrate interaction, following a pathway reminiscent of enzymes, see Part 2), ${ }^{11,13,200,273,274}$ iii) a partner for the construction of supramolecular bidentate ligand (ligand - ligand interaction, 25 Fig. 17), ${ }^{198,199}$ and iv) an additive that modifies the coordination spheres of metal phosphine complexes (ligand - ligand additive interaction). Hydrophobic interactions between a cyclodextrin and the remote part of a phosphine ligand coordinated to a metal complex can be beneficial or detrimental depending on the nature 30 of the catalytic system studied.

It has been recognized that randomly methylated $\beta$-cyclodextrin is able to form an inclusion complex with water-soluble phosphine ligands, leading to the dissociation of the ligand and the generation of low-coordinated metal species. ${ }^{275-277}$ For the rhodium-catalysed 35 hydroformylation of linear olefins, ${ }^{278}$ the effect is translated into an increase of the reaction rate but at the cost of the selectivity towards the linear aldehydes. ${ }^{276}$

To prevent the dissociation of the phosphine ligand, two strategies were employed: i) the group recognized by the 40 cyclodextrin was located far away from the phosphorus atom, ${ }^{279} \mathrm{ii}$ ) a chelating diphosphine, sulfoxantphos, was used that binds strongly the catalytic rhodium centre in water. ${ }^{280}$ In both cases, the cyclodextrin - phosphine complex remains associated during the catalytic process as demonstrated by the strong influence of the $\mathrm{CD}$ 45 additive on the selectivity of the reaction. The selectivity of the sulfoxantphos rhodium catalyst towards the formation of the linear aldehyde is increased in the presence of randomly methylated cyclodextrins in the hydroformylation of linear olefins. ${ }^{280}$ The electronic and steric influence of the cyclodextrin as well as 50 cyclodextrin - substrate interactions established within the second coordination sphere of the rhodium catalyst can explain this result. Leclercq et al. studied the complexation properties of
ARTICLE TYPE

dimethylated- $\beta$-CD $\quad(\mathbf{8 9}, \quad \beta$-DIME $) \quad$ and $\operatorname{di}(1-$ adamantyl)benzylphosphine $(\mathbf{9 0}, \mathrm{DABP}){ }^{281}$ In water, $\beta$-DIME 55 forms complexes with DABP through the inclusion of one or two of its adamantyl moieties (Fig. 26, a). Bis-ligated rhodium complexes containing the DABP ligand alone does not catalyse the hydroformylation of 1-octene probably because of the bulkiness of the ligand. Adding one equivalent of $\beta$-DIME generates a 60 monoligated rhodium phosphine catalyst that is active for the reaction (enforced dissociation of one cyclodextrin - DABP assembly). Additionally, the selectivity depends on the number of equivalent of $\beta$-DIME added, a higher selectivity being obtained with two equivalents of cyclodextrin additives. The authors ${ }_{65}$ suggest that the second equivalent of $\beta$-DIME forms an inclusion complex with the DABP ligand coordinated to the rhodium active species. The steric hindrance exerted by CD・DABP assembly favours the formation of the linear Rh-alkyl intermediate (see Fig. 26, a).

70 Application of phosphane-derived cyclodextrins in catalysis operating in water is usually hampered by the propensity of the phenyl groups located on the phosphorus atom to cap the cyclodextrin cavity. ${ }^{200}$ Recently, Monflier and co-workers took advantage of this property to reversibly modify the second 75 coordination sphere of a rhodium catalyst connected to the diphenylphosphine-modified CD 91 (Fig. 26, b) ${ }^{282}$ Based on twodimensional ${ }^{1} \mathrm{H}$ homonuclear NMR experiments and surface tension measurement in water, the authors proposed a schematic representation of 91 where the cyclodextrin is capped by one 80 phenyl group located on the phosphorus atom. However, the interaction is weak and the phenyl group is readily replaced by 1adamantanecarboxylate sodium salt (92). The rhodium catalysts derived from the inter inclusion rhodium complex (91•92) and the intra inclusion complex (91) exhibit different selectivity in the 85 hydroformylation reaction of methyl-4-pentenoate, a substrate that is not accommodated by the cyclodextrin cavity. Catalyst derived from 91.92 provides a higher selectivity towards the formation of the linear aldehyde as a probable result of strong steric repulsions between $\mathbf{9 1 . 9 2}$ and the branched Rh-alkyl intermediate.

\subsection{Modification of bidentate ligands}

The ligand - template strategy, mentioned above to modulate the properties of pyridyl-substituted monodentate ligands, has also been applied to bidentate ligands. ${ }^{283}$ Reek et al. prepared a diphosphine ligand, structurally-related to 83 (Fig. 24) in which 95 the dimethylamine group was replaced by $o-N$-methylanilinediphenylphosphine (93, Fig. 27). In hydroformylation catalysis, a single resting state is observed in which the diphosphine ligand coordinates to the $\mathrm{Rh}$ atom in an equatorial-apical fashion, the phosphoramidite ligand being located trans to the hydride (see 94). 100 Unlike what happened to monodentate 83, the presence of the template did not change the coordination mode, but the template did influence significantly the activity and the selectivity of the catalysts. Some bidentate hybrid ligands and their 
<smiles></smiles>

93
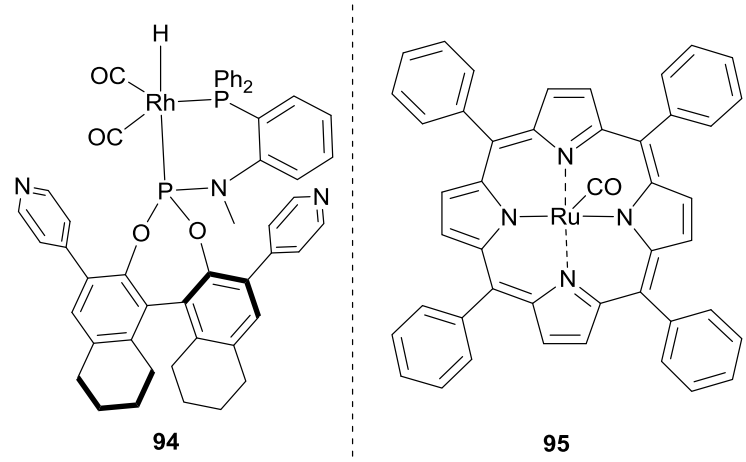

Fig. 27 Ligand - template strategy applied to bidentate phosphine ligands. The catalytic properties of the rhodium diphosphine complex 93 are drastically modified in the presence of various $\mathrm{Zn}^{\mathrm{II}}$ - or $\mathrm{Ru}^{\mathrm{II}}$-porphyrin templates. The catalyst which contain the self-assembled ligand $\mathbf{9 4}_{\mathbf{\bullet 9 5}} \mathbf{9 5}_{\mathbf{2}}$ was the more selective in asymmetric hydroformylation reaction of para-substituted styrenes. Ref: see the text.

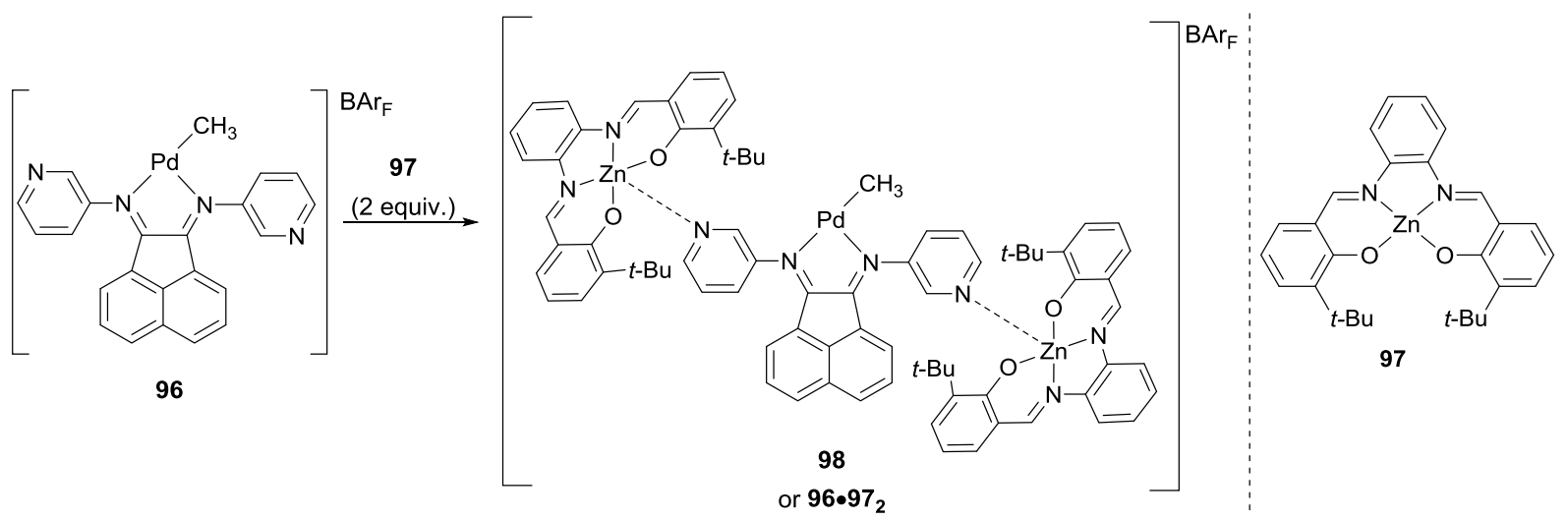

Fig. 28 Ligand - template strategy applied to bis-imine ligand. The binding of two equivalents of the salphen template $\mathbf{9 7}$ to the pyridyl groups of $\mathbf{9 6}$ yields $\mathbf{9 8}$, a very active and selective catalyst in $\mathrm{CO} / 4$-tert-butylstyrene copolymerization reaction. Ref: see the text.

respective ligand - template assemblies were evaluated in the asymmetric hydroformylation reaction of a series of para10 substituted styrenes. Self-assembled ligand 93.952 (Fig. 27) provided the best result; the branched aldehydes were obtained with good regioselectivity (b:1 ratio $>11.5$ ) and modest to good enantioselectivity $(59<\mathrm{ee}<82 \%, 4$ examples $)$.

The strategy is not restricted to diphosphine ligands. Bis-imine 15 Pd complex 96 containing pyridyl donor groups was modified with various salphen molecules and the influence of the resulting ligand - template assemblies was evaluated in $\mathrm{CO} / 4$-tert-butylstyrene copolymerization reaction (Fig. 28). ${ }^{268}$ The cationic palladium complex 96 alone, i.e. without salphen additives, was inactive, 20 probably due to intermolecular coordination of the free pyridine groups to palladium. In striking contrast, the catalyst $\mathbf{9 8}$ obtained by adding two equivalents of the salphen platform 97 to 96 was very active and selective in the copolymerization reaction. It produced the highest molecular weight copolymers $\left(M_{\mathrm{w}}=118 \times\right.$

$\left.2510^{3} \mathrm{~g} \cdot \mathrm{mol}^{-1}\right)$ reported at that time at room temperature without the use of the stabilising solvent $\mathrm{CF}_{3} \mathrm{CH}_{2} \mathrm{OH}$. Also the co-polymer obtained with catalyst $\mathbf{9 8}$ displayed a high degree of syndiotacticity with a percentage of $u u$ triads equals to $85 \%$.
Dydio et al. achieved a high level of stereocontrol in asymmetric 30 reactions as a result of efficient chirality transfer between a chiral additive and an achiral diphosphine ligand assembled through well-defined non-covalent interactions. ${ }^{284}$ The achiral diphosphine ligand 99 was designed which contained an anionic receptor in its backbone (Fig. 29). ${ }^{285,286}$ Upon addition of chiral anions, chiral 35 complexes are obtained which are tested in the asymmetric hydrogenation of various substrates. The efficiency of the catalyst strongly depends on the nature of the chiral anion (the "cofactor"). Cofactor $\mathbf{1 0 0}$ is one of the best chiral inducers; catalyst derived from 101 gave $98 \%$ ee for the asymmetric hydrogenation of 40 methyl-2-acetamidoacrylate (10). Molecular modelling of the assembled complex $[\mathrm{Rh}(\mathbf{9 9 \cdot 1 0 0})(\mathbf{1 0})]$ showed that one hydrogen bond is formed between the sulfur atom of the thiocarbonyl group of the chiral anion and the $\mathrm{N}-\mathrm{H}$ proton of substrate 10. Control experiments confirmed that the selectivity of the catalytic reaction 45 is dictated by this cofactor - substrate interaction.

On the one hand, supramolecular modification of ligands as described above gives an easy and rapid access to a large number of new, modified ligands. It allows for the tuning of the 


\section{Cite this: DOI: $10.1039 / \mathrm{c0xx00000x}$}

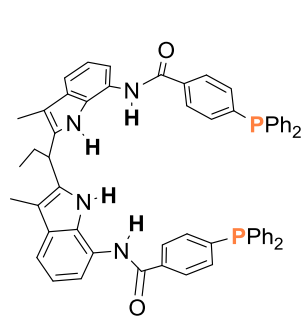

99 achiral precatalyst

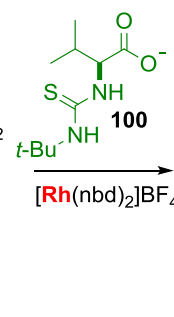

$\mathrm{NH}$ $\mathrm{NH}$ 100

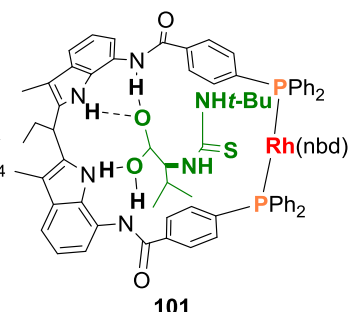

101

chiral precatalyst
$98 \%$ ee for the hydrogenation of 10

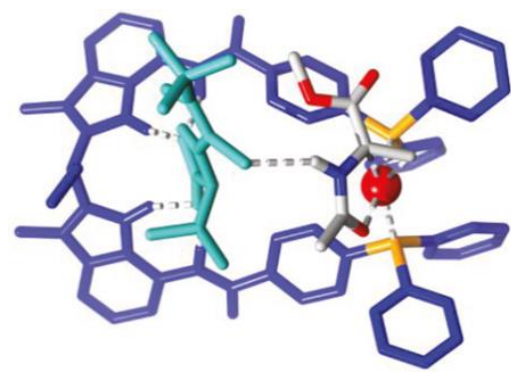

Fig. 29 Chirality transfer between an achiral diphosphine ligand and a chiral module. Above: modification of an achiral diphosphine ligand by a chiral anion that binds the receptor in the ligand backbone. Below: Molecular modelling structure of $[\operatorname{Rh}(\mathbf{9 9} \cdot 100)(10)]$. (The molecular model is reprinted with permission from ref. 284. Copyright 2011. American Chemical Society).

electronic, steric properties or the chiral environment of the 10 catalyst. On the other hand, recovery of a multi-component catalyst will be more complicated than that of a catalyst containing a covalent ligand. For most of the processes described above covalent systems with good performance have been reported and thus likely the new systems will not be practical for low-added15 value products.

\section{Catalyst - substrate interaction}

\subsection{Ligand - substrate interaction}

In enzymes, the entropic penalty associated with an optimal substrate(s) preorganization and a highly-organised transition state 20 is paid by the interplay of a set of secondary interactions established between the enzyme residues and the complementary groups borne by the substrate. These interactions, as well as the structural complexity of enzymes, explain the high rates and selectivities observed in enzymatic reactions. Such catalytic 25 proficiency is hardly achievable with artificial catalysts whose structures and mode of actions are far simpler compared to complex machinery exhibited by enzymes. However, two strategies exist to control the reactivity of a substrate: i) create a binding pocket that entraps the substrate near of the metal centre 30 (enzyme mimics, see Part 2), ${ }^{11}$ ii) design an attractive ligand substrate interaction that will direct the reactive part of the substrate at an ideal distance of the metal centre.

\subsubsection{General consideration}

Enhancement of the reaction rate by a ligand in metal catalysis can 35 be explained either by destabilisation of the ground state (the metal-ligand complex) or stabilisation of the transition state. ${ }^{287,288}$ (DHQD) 2 PYDZ (Fig. 30, a) and related bis-cinchona alkaloid compounds are very efficient ligands for the $\mathrm{OsO}_{4}$-catalysed asymmetric cis-dihydroxylation of olefins. Despite an intense 40 debate about the mechanistic basis of enantioselectivity for this reaction, ${ }^{289-291}$ strong evidence supports the formation of a binding pocket $^{287,289,292,293}$ suitable for the accommodation of hydrophobic substrates (such as styrene, ${ }^{287}$ 2-vinylnaphthalene, ${ }^{287}$ allylic ${ }^{292}$ and homoallylic $^{294}$ alcohol derivatives). $\pi-\pi$ stacking and van der ${ }_{45}$ Waals interactions between the substrate and the methoxyquinoline rings of the bis-cinchona ligand as well as edgeto-edge interaction between the substrate and the nitrogen atoms of the phthalazine or pyridazine spacer enable efficient transition state stabilisation. It accounts not only for the higher rate but also 50 for the extraordinary enantioselectivity displayed by this ligand. A representation of the pathway that leads to the observed enantiomer for the dihydroxylation of styrene is shown in Fig. 30, a.

The preference for one enantiomer over the other in asymmetric catalysis is usually explained by stereoinduction models: the 55 favoured reaction pathway is usually the one that minimizes steric repulsion and other non-attractive interactions between the incoming substrate or the metal-bound intermediate and the ligands during the transition state. The selectivity of a given catalytic reaction can also be dictated by weak attractive 60 interactions between the ligand and the substrate.

Evans and co-workers found that the result of the Diels-Alder reaction between $N$-crotonyl-2-oxazolidinone 102 (Fig. 30, b) and isoprene is strongly affected by the nature of the chiral auxiliaries. ${ }^{295}$ The chiral auxiliary bearing a benzyl instead of a ${ }_{65}$ cyclohexyl group provides higher selectivity at no cost of activity ( $d r=20.7: 1$ and 9.7:1, respectively). Control experiments nicely ascertained that this selectivity is a result of dipole-dipole and van der Waals attractions between the dienophile and the ligand's benzyl group. Later, Corey and co-workers investigated chiral 70 oxazaborolidines as catalysts for Diels-Alder reactions. They found that the selectivity is dictated by $\pi-\pi$ interactions between the electron poor dienophile (2-bromoacrylaldehyde) and the electron rich aromatic ring borne by the boron-based Lewis-acid catalyst (103, Fig. 30, c) ${ }^{296-298}$ Additional investigation on the 75 mode of action of oxazaborolidine catalysts pointed out the importance of an additional attractive interaction: a $\mathrm{C}-\mathrm{H} \bullet \bullet \mathrm{O}$ hydrogen bond occurring between the formyl group of the dienophile and the oxygen of the oxazaborolidine (see 103 and 104, Fig. 30, c). ${ }^{299}$ In these three examples (102-104), the addition 80 of the diene to the face of the dienophile that is not involved in non-covalent interactions led to a Diels-Alder product with high optical purity.

$\pi-\pi$ interactions also play a role in mediating the selectivity in rhodium-catalysed hydroformylation reactions of olefins. 

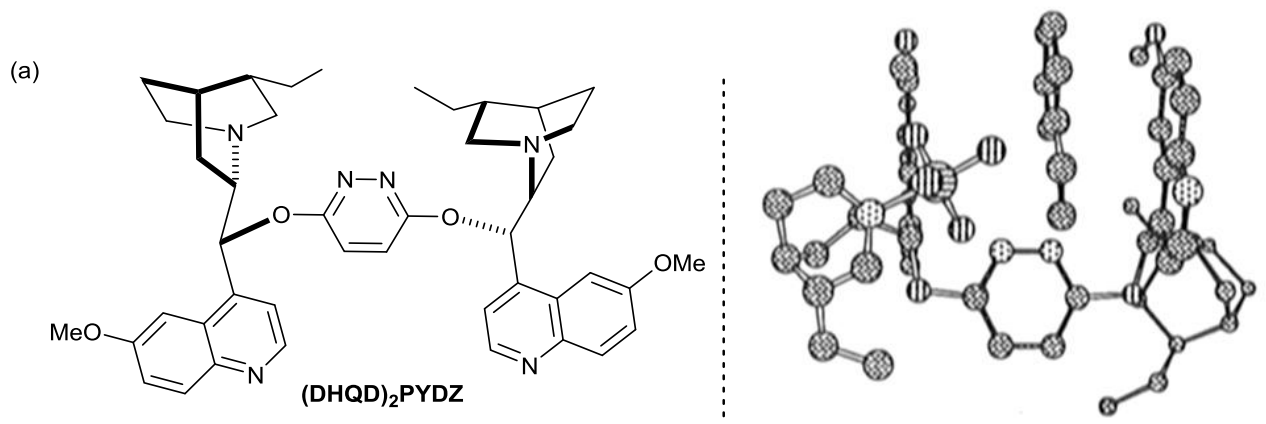

(b)

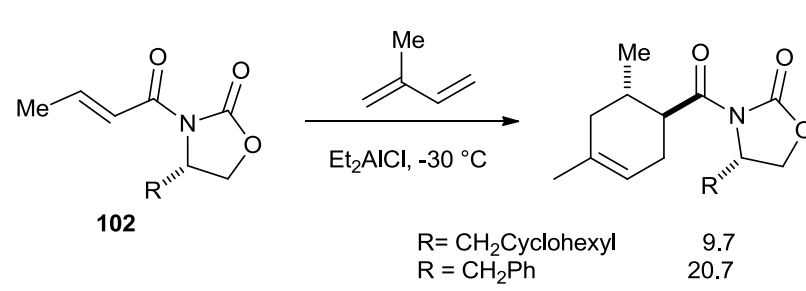<smiles>[R]C1COC(=O)N1C(=O)[C@@H]1CC=C(C)C[C@H]1[Y]I</smiles>

(c)

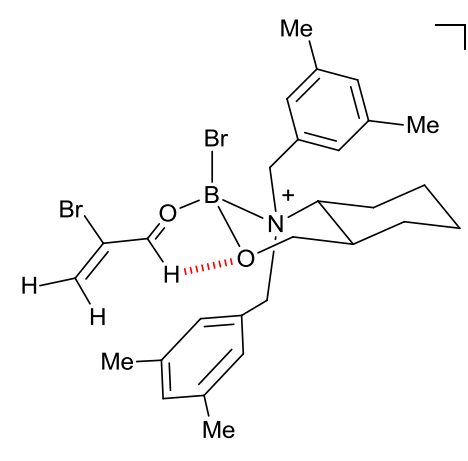

103<smiles></smiles>

104

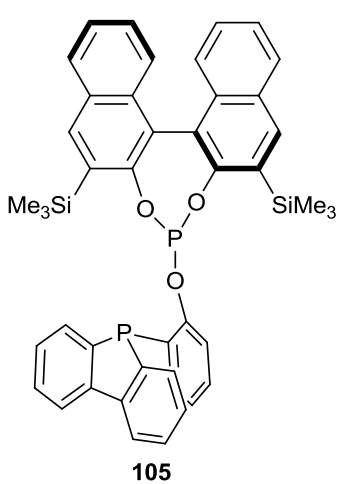

105<smiles>CC(C)(C)OC(=O)N1C[C@@H](p2c3ccccc3c3ccccc32)C[C@H]1Cp1c2ccccc2c2ccccc21</smiles>

106

5 Fig. 30 Example of weak attractive interactions between ligand and substrate in transition-metal complexes. (a) Left: (DHQD) 2 PYDZ gives high activity and selectivity for the $\mathrm{OsO}_{4}$-catalysed asymmetric dihydroxylation of olefins. Right: Representation of the complex of styrene, OsO $\mathrm{O}_{4}$ and $(\mathbf{D H Q D})_{2} \mathbf{P Y D Z}$

that leads to the observed enantiomer of styrene glycol via the [3 +2] cycloaddition pathway. The structure is reprinted with permission from ref. 289.

Copyright 1996. American Chemical Society). (b) The nature of the chiral auxiliary influences the selectivity of the reaction between $\mathbf{1 0 2}$ and isoprene.

Representation of the $\pi-\pi$ interaction that mediates the selectivity of the reaction. (c) The selectivity of Diels-Alder reactions catalysed by chiral

10 oxazaborolidine is explained by a combination of $\pi-\pi$ and hydrogen bond interactions (103 and 104). The dibenzophosphole moiety interacts with styrene and its derivatives through $\pi$ - $\pi$ interactions (105 and 106). DHQD = dihydroquinidine, PYDZ = pyridazine. Refs: see the text.

With the phosphite-phosphine hybrid ligand 105, a trend was found between the ee value obtained for the branched aldehyde products and the value of the Hammett constant $\sigma$ of the respective 15 4-substituted styrene substrates (Fig. 30, c). ${ }^{300}$ This was attributed to a $\pi-\pi$ interaction occurring between the $P$-aromatic rings of the phosphole moiety of $\mathbf{1 0 5}$ and the phenyl group of the styrene derivatives during the selectivity-determining step of the catalytic cycle. Such interaction between the dibenzophosphole moiety and 20 the substrate was established previously by computational studies of platinum-catalysed hydroformylation of styrene with the diphosphine ligand 106 (Fig. 30, c). ${ }^{301}$
The combination of hydrophobic interactions and weak attractive interactions allows for the encapsulation and the reaction 25 of substrate(s) within the inner space of well-defined molecular hosts (Part 2). ${ }^{11,14,16,46}$ Recently, the aromatic rings of welldesigned ligands have been used to create an aromatic $\pi$-wall surrounding the metal centre. The creation of such a $\pi$-pocket enables the discrimination and selective activation of aromatic 30 over aliphatic aldehydes of similar size. ${ }^{302,303}$ It suggests that the pocket efficiently recognizes aromatic substrates as a probable result of aromatic-aromatic interactions between the 


\section{Cite this: DOI: $10.1039 / \mathrm{c0xx00000x}$}

(a)

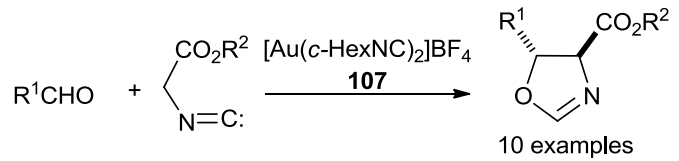
$87 / 13<$ trans/cis ratio $<100 / 0$ $87<\mathrm{ee}<97 \%$ (b)<smiles>NC(=Cc1ccccc1)C(=O)NC(Cc1ccccc1)C(=O)O</smiles>

109<smiles>N/C(=C\c1ccccc1)C(=O)N[C@@H](Cc1ccccc1)C(=O)O</smiles>

113

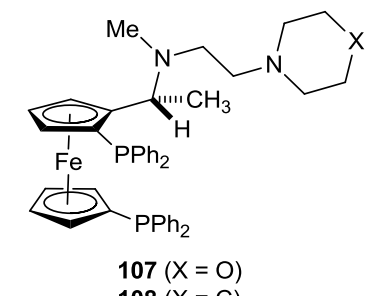<smiles>CC(C)OC(=O)[C@@H](Cc1ccccc1)NC(=O)C(Cc1ccccc1)NC(=O)[C@@H](Cc1ccccc1)C(C)C</smiles>

with 110 , de $=58 \%$ with 111, de $=90 \%$ with 112, de $=86 \%$

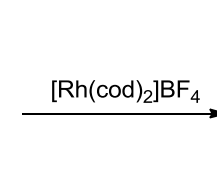<smiles>N[C@@H](Cc1ccccc1)C(=O)N[C@H](Cc1ccccc1)C(=O)O</smiles>

with 114 , de $=86 \%$ with $115 \mathrm{de}=82 \%$
$108(X=C)$

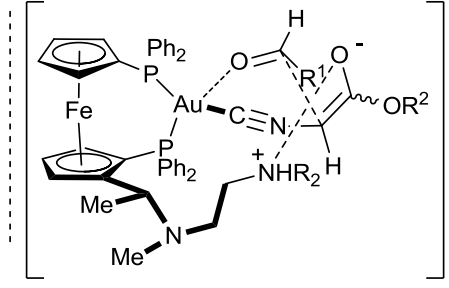<smiles>[R]N1C[C@H](OP)[C@H](OP)[C@@H]1Oc1ccccc1</smiles>

$\mathrm{R}=\mathrm{CH}_{2} \mathrm{CH}_{2} \mathrm{CH}\left(\mathrm{CH}_{3}\right)_{2}$ (110) $\mathrm{R}=\mathrm{CH}_{2} \mathrm{CH}_{2} \mathrm{NMe}_{2}$ (111)

$\mathrm{R}=\mathrm{CH}_{2} \mathrm{CH}_{2} \mathrm{CH}_{2} \mathrm{NMe}_{2}$ (112)<smiles>[R16]OC1CN([R])C[C@H]1O[Ga+]</smiles>

$$
\mathrm{Ar}=4-\mathrm{OMe}-\mathrm{C}_{6} \mathrm{H}_{4}
$$

$\mathrm{R}=\mathrm{CH}_{2} \mathrm{CH}_{2} \mathrm{CH}\left(\mathrm{CH}_{3}\right)_{2}$ (114)

$\mathrm{R}=\mathrm{CH}_{2} \mathrm{CH}_{2} \mathrm{NMe}_{2}$ (115)

Fig. 31 Examples of bifunctional metal catalysts with remote basic tertiary amines present on the ligand backbone. (a) The terminal amine is protonated in presence of the isocyanoacetate substrate and the resulting ammonium and enolate are ion-paired. This electrostatic interaction restricts the degree of conformational freedom of the enolate and favours the addition on the $S i$ face of the enolate. (b) The terminal amino group of ligands $\mathbf{1 1 1}$ and $\mathbf{1 1 2}$ has a positive effect on the selectivity obtained in the rhodium-catalysed hydrogenation of $\mathbf{1 0 9}$. This effect is not observed in the hydrogenation of $\mathbf{1 1 3}$, the enantiomer of 109. de = diastereomeric excess. Refs: see the text.

phenyl rings of the catalyst and the aromatic ring of the aldehyde.

In the preceding examples, the attractive interactions at the origin of the enhanced rate and selectivity were usually determined 10 a posteriori based on trial-and-error ligand screening cycles and experimental data (X-ray structures, use of Hammett constants, etc...). In the following examples, a degree of ligand design was rationally incorporated and a better control and understanding of the ligand - substrate interactions were thus achieved.

\section{5.1.2 Bifunctional metal catalysts}

5.1.2.1 Metal ligands with a proton donor/acceptor moiety

As a functional group of the ligand interacted with the substrate during the catalytic cycle, the term "metal ligand bifunctional catalysis"2 was used to imply that, in addition to the metal centre 20 of the catalyst, a function plays a role in the catalytic cycle. As a major general definition, "bifunctional catalysis" indicates that more than one functional group in the catalyst is involved in the rate-determining step, leading to the observed rate enhancement. ${ }^{304}$ The functional catalysts can be: a) an organic group tethered to a 25 metal centre, b) an organocatalyst with two or more catalyticallyactive organic functions (see section 5.2) and c) homo- or heteromultimetallic complexes that simultaneously activate two reaction partners. Prototypical examples of the latter case are the multimetallic systems developed by Shibasaki ${ }^{305-309}$ and 30 Jacobsen's catalysts ${ }^{310}$ for the asymmetric ring opening of epoxides with nitrogen nucleophiles. According to our general definition of supramolecular catalysis, this topic will not be included here. ${ }^{305-315}$

For catalytic systems related to case a, the mode of activation of 35 the substrate will depend on the nature of the function located on the ligand (Brønsted base, Lewis base, Lewis acid, ion, hydrogen bond donor/acceptor). In many examples of metal bifunctional catalysts incorporating a Lewis base, the base coordinates to a metal/metalloid centre $307,309,312-314,316$ (that further activates the 40 substrate) or directly reacts with a substrate; ${ }^{304,317}$ accordingly these examples will not be described here. In a few cases, the base acts as a hydrogen bond acceptor for the substrate and then reacts with it (proton transfer). ${ }^{318}$ The resulting weak acid is a hydrogen bond donor that can interact with the same or a different substrate.

${ }_{45}$ Bifunctional catalysts which possess a function acting as a proton donor/acceptor will be mentioned in this subsection. In the next subsection (5.1.2.2), we will describe examples of bifunctional metal catalysts that interact with the substrate via secondary interactions (hydrogen bond and/or electrostatic interactions) 50 without forming a new bond. Bifunctional metal catalysts that incorporate both a hard and a soft (metal centre) Lewis acid have found very few applications. ${ }^{319,320}$ Metalloenzyme mimics in which host molecules are covalently linked to metal catalysts will be described in Part 2. ${ }^{11}$

55 Ito, Sawamura and Hayashi reported avant la lettre bifunctional catalysts that operate through secondary interactions between the remote parts of chiral ligands and the substrate. ${ }^{321}$ In 1986, they 
found that chiral ferrocenylphosphine bearing a tertiary amino group at the terminal position of the pendant chain are very efficient ligands for the gold-catalysed asymmetric condensation of isocyanoacetate with aldehydes (Fig. 31, a). ${ }^{322}$ Notably, catalyst 5 derived from ligand $\mathbf{1 0 7}$, which possesses a terminal morpholino group, provided the trans-oxazoline products with high selectivity. ${ }^{322-325}$ Control experiments with analogues of 107: i) lacking the amino group, ii) with a hydroxyl instead of an amino pendant group, iii) with a longer tether between the amino group 10 and the ferrocene moiety; are less efficient ligands for the reaction. In the proposed transition state, the electrostatic interaction between the enolate substrate, coordinated to the gold atom through the isocyano group, and the ammonium pendant group of the ligand governs the facial selectivity (enolate-Si face attack). 15 The same sense of stereoselection is observed with bulkier enolates. It indicates that the selectivity is probably determined by the attractive interaction between the ammonium and the enolate rather than by the steric repulsion between the substituent of the enolates and the ligands of the Au complex. Ligand $\mathbf{1 0 8}$ is more 20 efficient than ferrocenylphosphine ligands lacking the terminal amino group in the rhodium-catalysed hydrogenation of fully substituted acrylic acids. ${ }^{326}$ Electrostatic interaction between the carboxylate group of the substrate and the pendant ammonium group of $\mathbf{1 0 8}$ is thought to govern the enantioface differentiation of 25 the olefin.

Rhodium catalysts derived from the chiral diphosphonite ligands $\mathbf{1 1 1}$ and $\mathbf{1 1 2}$ are selective for the asymmetric hydrogenation of the dehydrodipeptide 109. ${ }^{327-329}$ The pendant tertiary amino group seems to play a key role in mediating the 30 selectivity of the reaction since the analogous ligand $\mathbf{1 1 0}$ (with $\mathrm{CH}\left(\mathrm{CH}_{3}\right)_{2}$ pendant group instead of $\left.\mathrm{NMe}_{2}\right)$ is less selective. On the contrary, for the hydrogenation of the dehydrodipeptide 113, (the enantiomer of 109) the electrostatic interaction is not necessary since ligands lacking the terminal amino groups also provide high 35 degree of stereoinduction (compare ligands 114 and 115, Fig. 31, b).

Even though some mechanistic details are lacking for these seminal bifunctional catalysts, these examples clearly demonstrated that the presence of a basic site in the ligand 40 backbone can be beneficial in order to activate the substrate and insure an optimal orientation of the substrate prior to reaction.

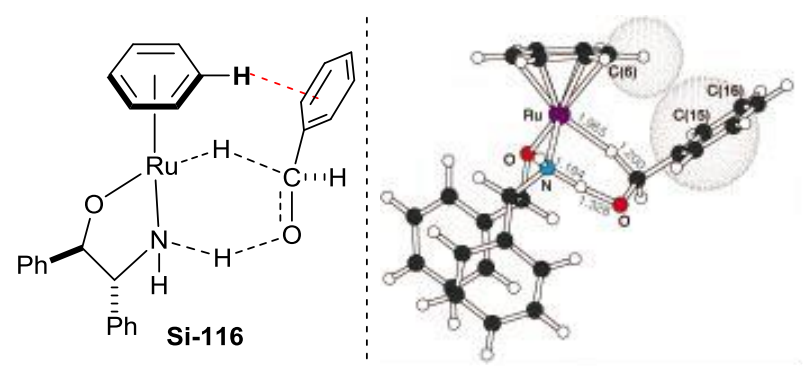

Fig. 32 Origin of the enantioselectivity in the transfer hydrogenation 45 reaction with Noyori catalysts. The optimised geometry of Si-116 at the DFT level reveals a $\mathrm{C}-\mathrm{H}-\pi$ attractive interaction between the $\eta^{6}$-benzene ligand coordinated to the Ru centre and the substrate (benzaldehyde). (The optimised geometry is reprinted with permission from ref. 332. Copyright 2001. John Wiley and Sons).
50 In 1995, Noyori and co-workers found that ruthenium complexes containing both diphosphine and ethylenediamine ligands are very active catalysts for the hydrogenation of ketones. ${ }^{58}$ Chelating diamines lacking the hydrogen bond donor $\mathrm{N}-\mathrm{H}$ groups do not accelerate the reaction compared to $\left[\mathrm{RuCl}_{2}\left(\mathrm{PPh}_{3}\right)_{3}\right]$. The 55 same "N-H effect" was found for asymmetric transfer hydrogenation reactions performed with the Noyori-Ikariya catalyst $\left\{\operatorname{RuCl}\left(\eta^{6}\right.\right.$-benzene $)[(S, S)$-TsDPEN $\left.]\right\}$ (Ts = tosyl, DPEN = diphenylethylenediamine). ${ }^{59,60}$ This effect was rationalized by the presence of a hydrogen bond established between one $\mathrm{N}-\mathrm{H}$ proton ${ }_{60}$ of the amine ligand and the carbonyl oxygen of the substrate. $\left[\mathrm{Ru}\left(\eta^{6}\right.\right.$-benzene $\left.)\left(\mathrm{NHCH}_{2} \mathrm{CH}_{2} \mathrm{Y}\right)\right](\mathrm{Y}=\mathrm{O}$ or $\mathrm{NH})$ dehydrogenates the alcohol substrate via an $\mathrm{O} \cdot \bullet \mathrm{H}^{\cdots} \mathrm{N}$ hydrogen bond and the resulting $\left[\mathrm{Ru}(\mathrm{H})\left(\eta^{6}\right.\right.$-benzene $\left.)\left(\mathrm{NH}_{2} \mathrm{CH}_{2} \mathrm{CH}_{2} \mathrm{Y}\right)\right]$ complex activates the ketone via a $\mathrm{C}=\mathrm{O} \bullet \bullet \mathrm{H}-\mathrm{N}$ hydrogen bond. ${ }^{330,331}$ Both steps ${ }_{65}$ proceed via six-membered transition states involving one hydrogen bond interaction between the Ru ligand and the substrate. Interestingly, in transition states involving Noyori catalyst $\mathbf{S i - 1 1 6}$ and aromatic aldehyde substrates, an attractive $\mathrm{C}-\mathrm{H}-\pi$ interaction between the arene substituent of the complex and the aryl 70 substituent of the substrate is at the origin of enantioselectivity (Fig. 32) ${ }^{332}$ Since the initial discovery of Noyori and co-workers, a plethora of metal catalysts (mainly $\mathrm{Ru}, \mathrm{Rh}$ and $\mathrm{Ir}$ and in a few cases $\mathrm{Fe}^{333}$ and $\mathrm{Os}^{334-340}$ ) have been described that incorporate amino $\left(\mathrm{NH}_{2}\right)$ or amido $(\mathrm{NH})$ ligands for hydrogenation, 75 dehydrogenation or transfer hydrogenation catalysis., 2,60,341-344 The metal-coordinated amino/amido groups are at an ideal position for the cooperative activation of $\mathrm{H}_{2}, i-\mathrm{PrOH}$ or $\mathrm{C}=\mathrm{X}(\mathrm{X}=\mathrm{O}, \mathrm{N})$ bonds; only in a rare case the $\mathrm{N}-\mathrm{H}$ group was not directly linked to the metal. $^{345}$ In most cases, a mechanism similar to Noyori catalysts 80 was proposed (i.e. the involvement of six-membered transition states $)^{1,330,331,346-351}$ for the hydrogenation reaction. ${ }^{352}$ For a more detailed description of metal bifunctional catalysts exhibiting a "N-H effect" in catalysis, we guide the reader to the excellent recent review by Ding and co-workers. ${ }^{3}$

85 The Noyori-Ikariya catalyst and related complexes also accelerate the conjugate addition of carbanions to various electrophiles. ${ }^{353-361}$ The amido ligand deprotonates the pronucleophile and the resulting amino moiety serves as hydrogen bond donor to direct the nucleophilic addition towards the metal90 coordinated electrophile (similarly to aldol reactions performed with 107, Fig. 31). ${ }^{362}$ Recently, Wang and co-workers found that chiral TF-BiphamPhos derivatives are very efficient ligands for the transition-metal catalysed asymmetric reactions of various activated alkenes with azomethine ylides (Fig. 33). ${ }^{363}$ These 95 authors proposed a mechanism for the $\mathrm{Cu}^{\mathrm{I}}$-catalysed Michael reaction between alkylidene bisphosphonates (117) and azomethine ylides (118). ${ }^{363}$ The active species is a $\mathrm{Cu}^{\mathrm{I}}$ complex containing the TF-BiphamPhos ligand and deprotonated 118 acting as a $(N, O)$-chelate. The nucleophilic addition of the active 100 species to the electrophile $\mathbf{1 1 7}$ generates the carbanionic intermediate 119 which is stabilized by a hydrogen bond established between the $\mathrm{P}=\mathrm{O}$ oxygen atom of the phosphonate group and one $\mathrm{N}-\mathrm{H}$ proton of the chiral ligand. The hydrogenbound proton then migrates to the carbanion yielding the neutral 105 intermediate 120. Coordination of $\mathbf{1 1 8}$ to the copper centre regenerates the active species. The proton acceptor/donor role of 


\section{Cite this: DOI: 10.1039/c0xx00000x}

\section{www.rsc.org/xxxxxx}

ARTICLE TYPE

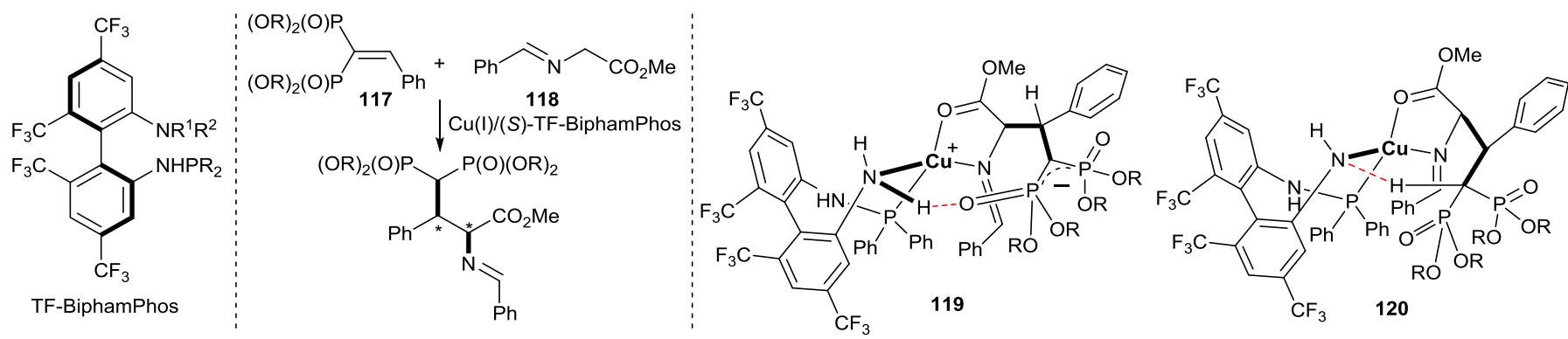

Fig. $33 \mathrm{Cu} / \mathrm{TF}-B i p h a m P h o s$ bifunctional catalysis for the Michael addition between alkylidene bisphosphonate 117 and azomethine ylide 118 . The amino moiety, coordinated to the copper atom, acts as a hydrogen bond donor/acceptor during the catalytic cycle. Refs: see the text.

the nitrogen ligand is consistent with the " $\mathrm{N}-\mathrm{H}$ effect" found 5 experimentally. ${ }^{364}$

Pyridine and tertiary amine moieties have hydrogen acceptor capabilities that can be beneficial for bifunctional catalysis. Whether these groups also behave as a base, very precise mechanistic studies are required since proton exchange processes 10 are hardly detectable. ${ }^{365}$ Heterocyclic containing phosphines, e.g. 2-tert-butyl-o-pyridyl-diphenylphosphine or 2-tert-butyl-oimidazol-diphenylphosphine, are the simplest bifunctional ligands, as they contain a phosphorus ligating group capable of forming stable metal complexes and a $s p^{2}$ nitrogen atom, which can form 15 hydrogen bonds with an adjacent ligand or an incoming substrate. For example, the $o$-pyridyl-diphenylphosphine ligand facilitates the heterolytic cleavage of $\mathrm{H}_{2}$ through hydrogen interactions and subsequent proton transfer. ${ }^{366}$

Grotjahn and co-workers found that $\mathbf{1 2 1}^{367,368}$ and $\mathbf{1 2 2}^{365}$ (Fig. 2034 , a) are very efficient catalysts for the anti-Markovnikov hydration of alkynes providing aldehydes with high selectivity and activity. Compared to $[\mathrm{Ru}(\mathrm{Cl}) \mathrm{Cp}(\mathrm{dppm})]$, the most active catalyst at the beginning of the Grotjahn's studies, ${ }^{369} \mathbf{1 2 1}$ and $\mathbf{1 2 2}$ are 2500and 90 -fold more active respectively. $\mathbf{1 2 1}$ and $\mathbf{1 2 2}$ are the resting 25 states; within these complexes the water molecule is surrounded by the two phosphine ligands and is hydrogen-bonded to the nitrogen atoms. ${ }^{365}$ The alkyne-metal $\pi$ complex $\mathbf{1 2 3}$ has been identified as an intermediate in the catalytic reaction, the coordinated alkyne being engaged in non-classical hydrogen bond interactions with 30 the surrounding nitrogen atoms. Another interaction has been identified within the Ru acyl complex 125 that features a hydrogen bond between the $\mathrm{N}-\mathrm{H}$ proton of the pyridinium moiety of the ligand and the carbonyl oxygen of the coordinated acyl group. Complex $\mathbf{1 2 5}$ is formed by the addition of water to the vinylidene 35 124, another intermediate that has been spectroscopically identified. Following these in-depth mechanistic studies, the role of the pyridine or imidazole moieties has been clarified: i) they facilitated the alkyne-to-vinylidene transformation (from $\mathbf{1 2 3}$ to 124), and ii) they assisted the addition of water towards the 40 vinylidene complex (from 124 to 125). ${ }^{368,370-372}$ Breit ligands based on the 2-amidopyridine and isoquinolone recognition units (Fig. 6) were investigated for the ruthenium-catalysed hydration of nitriles. ${ }^{121}$ If ligand - ligand interaction has been ascertained in the solid-state of the precatalyst, this interaction is probably lost at the
45 expense of ligand - water interaction occurring during the catalytic reaction.

Using the same methodology, bifunctional catalysts have been designed for the hydration of nitriles. The neutral Ru complex 126 (Fig. 34, b) provides the amide products in high yield but requires 50 high temperature $\left(180{ }^{\circ} \mathrm{C}\right) \cdot{ }^{373} \mathrm{In}$ the postulated key intermediate 127, the pyridyl group of the phosphine ligand directs the water molecule in close proximity to the coordinated nitrile. Previous results with $\left[\mathrm{Ru}(\mathrm{H})\left(\eta^{5}-\mathrm{C}_{9} \mathrm{H}_{7}\right)(\mathrm{dppm})\right]$ (active at $120{ }^{\circ} \mathrm{C}$ ) showed that the hydride ligand play the same role, i.e. it established a 55 hydrogen bond with the incoming water molecule which directs the addition of the oxygen lone pair to the $\mathrm{Ru}$-coordinated nitrile substrate. ${ }^{374}$ Breit ligands were also investigated for this reaction. ${ }^{122}$ In the solid state structure of the best precatalyst, the cis-coordinated 3-diphenylphosphinoisoquinolone ligands formed 60 a hydrogen bond with the neighboring acac co-ligand (128, Fig. 34, b) and not between each other as presented in section 3.1.2 (Fig. 5). A hydrogen bond interaction between the carbonyl group of the isoquinolone moiety and the reacting water has been hypothesized to explain the good activity observed with this class 65 of ligands. Very recently, Bera and co-workers reported the use of a NHC derivative tethered with a 1,8-naphthyridine group as a ligand in the rhodium-catalysed hydration of organonitriles at 25 ${ }^{\circ} \mathrm{C}$ (TOF up to $20,000 \mathrm{~h}^{-1}$ for acrylonitrile). ${ }^{375}$ DFT calculations suggest the formation of a hydrogen bond between the 1,870 naphthyridine group of the NHC ligand and a water molecule coordinated to the Ru atom (see 129, Fig. 34, b). Compared to 126, this bifunctional catalyst not only directs the oxygen lone pair for nucleophilic attack to the substrate but also activates the water molecule through the combined effect of metal ion coordination 75 and the hydrogen bond interaction.

Grotjahn and co-workers found that catalyst 130 (Fig. 34, c), with 2-tert-butyl-o-imidazol-di-tert-butyl-phosphine acting as a $(P, N)$-chelate, is able to isomerise alkenes over several carbon bonds providing exclusively the $(E)$-isomer products. ${ }^{376,377}$ In this 80 original catalyst, the imidazole group plays a crucial role because it acts as an internal base for the deprotonation of allylic hydrogen. Whether a hydrogen bond interaction is involved in such a process has not been probed. An iridium complex bearing one $o$-imidazoldi-isopropyl-phosphine ligand was shown to 


\section{Cite this: DOI: $10.1039 / \mathrm{c0xx00000x}$}

\section{www.rsc.org/xxxxxx}

ARTICLE TYPE
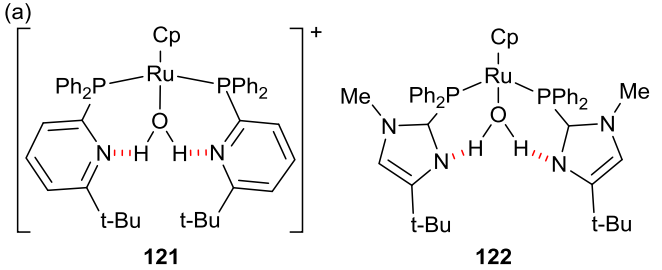

(b)<smiles></smiles>

126

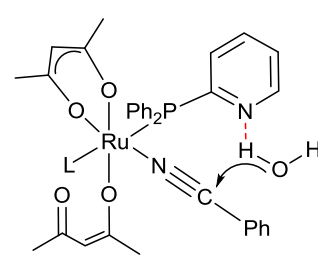

$\mathrm{L}=\mathrm{PhCN}$ or $\mathrm{PPh}_{2} \mathrm{Py}$ 127

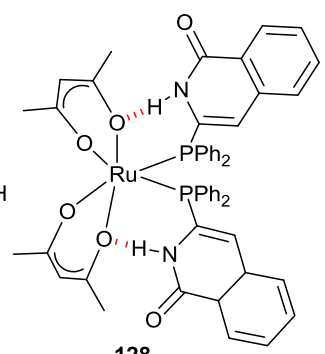

128

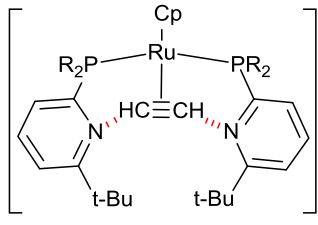

123

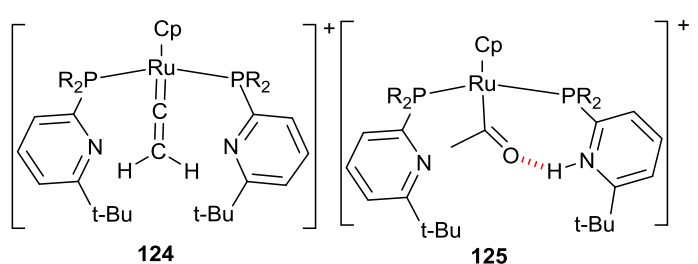

124

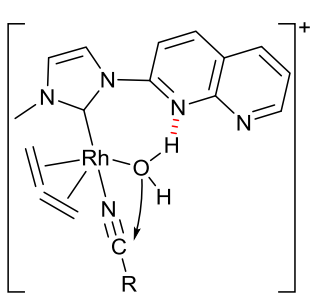

(c)

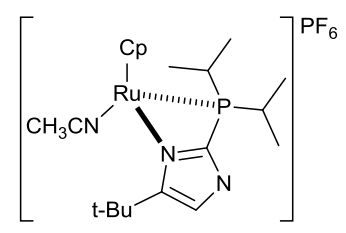

(d)

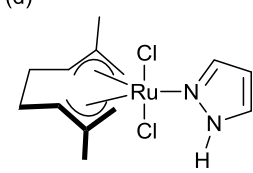

131

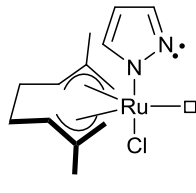

132

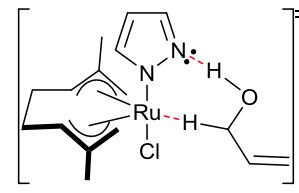

133

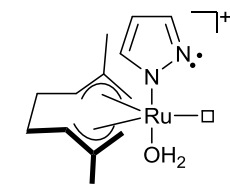

134
129

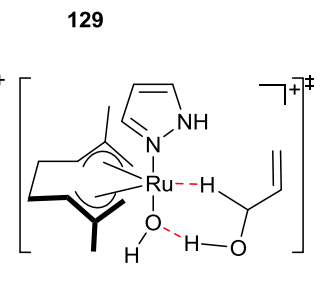

135

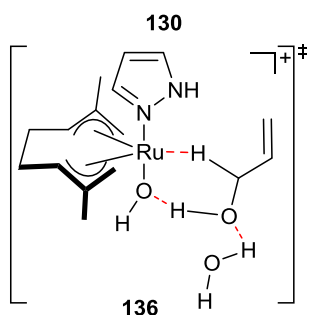

Fig. 34 Bifunctional catalysts combining a Lewis acid (the transition-metal) and a base (proton acceptor) for the anti-Markovnikov hydration of alkynes (a), the hydration of nitriles (b), the isomerisation of alkenes (c) and the isomerisation of allylic alcohols (d). Refs: see the text.

catalyse the transfer hydrogenation of cyclohexanone; however the 5 acceleration provided by the P-N ligand was modest. ${ }^{378}$ Pyrazole and protic-NHC complexes ${ }^{345,379}$ have a $\mathrm{N}-\mathrm{H}$ group located at the $\beta$-position to the metal, however their use as bifunctional catalysts has been poorly demonstrated yet. ${ }^{380}$ Pyrazole-based ruthenium(IV) complex $\mathbf{1 3 1}$ catalysed the isomerisation of 10 substituted allylic alcohols into ketones in water at $75{ }^{\circ} \mathrm{C}$ in the absence of base (Fig. 34, d) ${ }^{381}$ DFT calculations did not ascertain if the active species is the unsaturated neutral Ru chloride complex 132 or the cationic Ru-aqua complex 134, however the latter seems more likely in water. The main steps of the catalytic cycle are the 15 dehydrogenation of the alcohol and the hydrogenation of the resulting $\alpha, \beta$-unsaturated ketone. Whatever the active species may be (132 or 134), the dehydrogenation involves a six-membered ring transition state similar to the one found with the Noyori catalysts (133 and 135). The difference arises from the nature of 20 the Lewis base involved in the interaction: a pyrazolide or a hydroxo ligand for $\mathbf{1 3 3}$ and $\mathbf{1 3 5}$ respectively. The resulting pyrazole and water molecule are also involved in the hydrogenation step: the 1,4-addition of the $\mathrm{Ru}-\mathrm{H}$ hydride and $\mathrm{N}-$ $\mathrm{H}$ or $\mathrm{O}-\mathrm{H}$ protons to the $\alpha, \beta$-unsaturated ketone. Interestingly, the 25 energy of the transition state $\mathbf{1 3 5}$ is lowered when a water molecule is coordinated to the oxygen atom of the substrate (see 136, Fig. 34 , d). The ability of water molecules to accelerate organic reactions through hydrogen bonding is well-known e.g. for DielsAlder reactions ${ }^{382}$ and on-water catalysis ${ }^{383}$ but a similar role for
30 transition-metal catalysis is rarely envisaged. ${ }^{384-386}$

The proton donor/acceptor abilities of pendant basic/acidic groups are at the origin of the high activity and lower overpotential obtained with $\mathrm{Ni}$ and Co electrocatalysts for hydrogen production and oxidation. ${ }^{387,388}$ Notably, Dubois and co-workers found that a 35 series of nickel complexes incorporating pendant basic groups in their ligand backbone, such as 137 (Fig. 35), are more efficient electrocatalysts than $[\mathrm{Ni}(\mathrm{depp})]^{2+} \quad(\mathrm{depp}=$ bis(diethylphosphino)propane). Mechanistic studies clarify the roles played by the base during hydrogen oxidation: i) it facilitates 40 the heterolytic cleavage of $\mathrm{H}_{2}$ and, ii) it promotes the intra-and intermolecular proton transfer in solution. The $\mathrm{R}$ and $\mathrm{R}^{1}$ groups of the $\mathrm{Ni}$ complex 137 can be tuned in order to make of 137 a hydrogen production or a hydrogen oxidation catalyst. The addition of $\mathrm{H}_{2}$ towards the $\mathrm{Ni}^{\mathrm{II}}$ complex 138, which contain four 45 pendant tertiary amines, yields 140 , a tetrahedral $\mathrm{Ni}(0)$ complex for which one amine of each ligand has been protonated. ${ }^{389}$ DFT calculations suggest that complex $\mathbf{1 3 9}$ is the non-observed intermediate in which the dihydrogen molecule is stabilised by two adjacent tertiary amines. The high activity of the Ni catalyst $\mathbf{1 3 8}$ 50 for hydrogen oxidation (TOF of $\left.10 \mathrm{~s}^{-1}\right)^{390}$ is probably a result of this interaction as well as facilitated proton and electron distribution between the Ni centre and the pendant basic groups. Subsequent mechanistic studies support the hypothesis than two positioned pendant basic groups in the 


\section{Cite this: DOI: 10.1039/c0xx00000x}

\section{www.rsc.org/xxxxxx}

ARTICLE TYPE

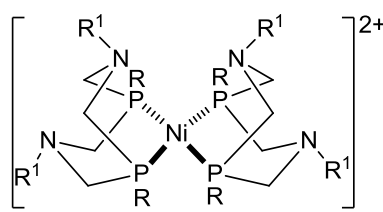

137

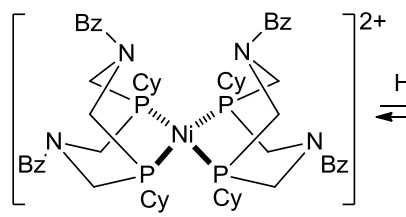

138

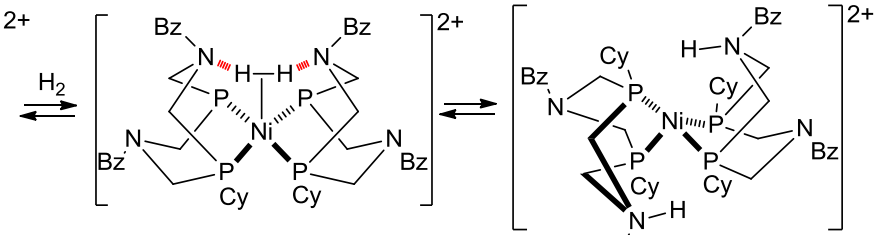

139
140

Fig. 35 Nickel complexes with pendant tertiary amines as catalysts for the production and oxidation of hydrogen. depp = bis(diethylphosphino)propane. Refs: see the text.

a)

a)

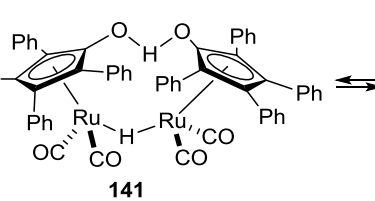

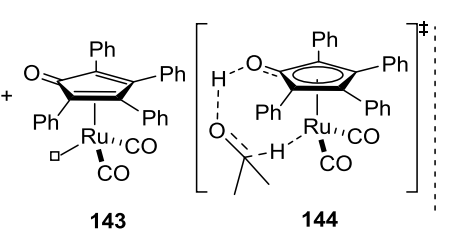

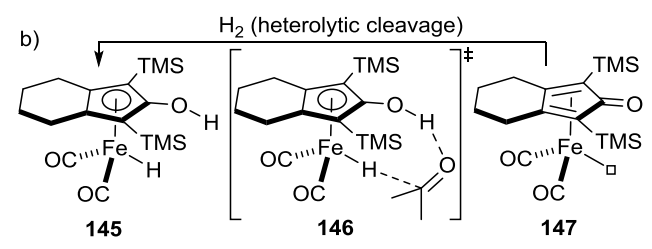

Fig. 36 Bifunctional catalysts with a pendant oxygen base: Shvo catalyst (a) and Casey catalyst (b). Refs: see the text.

second coordination sphere of metal complexes are important for achieving high catalytic rates with $\mathrm{Ni}$ catalysts ${ }^{391}$ whereas only one pendant base is needed with Co catalyst. ${ }^{392}$ Although most of the bifunctional catalysts reported to date possess nitrogen 10 groups acting as proton donor/acceptor sites, a few catalysts exist that possess basic functions based on oxygen moieties. $\left[\mathrm{Pd}(\mathrm{OAc})_{2}\left(\mathrm{H}_{2} \mathrm{O}\right)(\mathrm{IiPr})\right]$, for which the water molecule is hydrogenbonded by the surrounding acetate ligands, is a precatalyst for the aerobic oxidation of alcohols (with $\operatorname{IiPr}=1,3-\operatorname{bis}(2,6-$ 15 diisopropylphenyl)imidazol-2-ylidene). ${ }^{393,394}$ One acetate ligand acts as an internal base that deprotonates the alcohol substrate probably through the intermediacy of a hydrogen-bonded species. The Shvo catalyst is another example of bifunctional catalyst that possesses an oxygen group acting as a proton donor/acceptor 20 site. ${ }^{395,396}$ Upon heating, the bimetallic dimer 141 produces the mononuclear complexes 142 and 143, which are active species for the hydrogenation of unsaturated substrates and the dehydrogenation of saturated substrates respectively (Fig. 36, a). Among the possible reaction pathways, ${ }^{397-399}$ a concerted outer25 sphere mechanism, similar to the one taking place with Noyori catalysts, seems privileged for the transfer hydrogenation of ketones $^{98}$ and imines (see 144, a possible transition state for the transfer hydrogenation of ketones with the Shvo catalyst, Fig 36, a). ${ }^{99,400}$ Casey et al. reported that the Knölker iron complex ${ }^{401} \mathbf{1 4 5}$ 30 catalyses the hydrogenation of ketones (Fig. 36, b) ${ }^{402}$ Again, the hydrogenation of carbonyl substrates follows a concerted outersphere mechanism in which the two hydrogen atoms attached to the $\mathrm{Fe}$ and $\mathrm{O}$ atoms of the catalyst are transferred to the oxygen and carbon atoms of the carbonyl compound (see transition state ${ }_{35}$ 146). ${ }^{403} \mathrm{H}_{2}$ is cleaved heterolytically by the resulting unsaturated $\mathrm{Fe}(0)$ complex 147 through the intermediacy of a stable $\eta^{2}-\mathrm{H}_{2}$ complex. Anionic SPO ligands (Fig. 1) are another category of bifunctional catalysts in which a remote oxygen group plays the role of the base. ${ }^{97}$
40 Breit and co-workers illustrated how a remote basic group located in the ligand backbone can be used to control the selectivity of a catalyst. ${ }^{404,405}$ They found that the acylguanidinefunctionalized phosphine 148 and 149 (Fig. 37, a) are very efficient ligands in the rhodium-catalysed hydroformylation of 45 vinylacetic acid and other $\beta, \gamma$-unsaturated acids yielding preferentially the linear aldehydes. ${ }^{404}$ In a simplified version of the reaction pathway, a guanidinium-carboxylate ion pair is formed which directs the double bond of the substrate towards the $\mathrm{Rh}-\mathrm{H}$ bond, leading to the metalation of the rhodium at the $\gamma$-position of 50 the carboxylate anion. Proton transfers between the substrate and the guanidine moiety borne by the ligands occurs spontaneously during the course of the catalytic reaction. The synthetic utility of the catalyst is also demonstrated by its ability to transform selectively the double bond located in the 2,3 position of the 55 carboxylic group, in contrast to the other double bonds present in the molecule (see e.g. the chemoselective transformation of substrate 150). This result indicates that, for $\beta, \gamma$-unsaturated acids, the ligand - substrate interaction brings the double bond at an ideal position for the accomplishment of the subsequent rate${ }_{60}$ determining hydrometalation step. The precise nature of the ligand - substrate interaction and its exact role in the different steps of the catalytic cycle are difficult to ascertain according to the numerous possible intermediates. DFT calculations of the hydrometalation step suggest that $\mathbf{1 5 2}$ is a possible transition state for which four 65 hydrogen bonds are established between the carboxylate anion and two guanidine moieties (one protonated, one neutral). ${ }^{405}$ The hydrogen bonds point directly towards the lone pair of the carboxylate. The complexation of the anion seems more favoured with two instead of one guanidine moiety. The complexation role 70 of the guanidine is probably not limited to the metalation step and its participation in the other catalytic steps is surmised. When $\alpha, \beta-$ unsaturated 


\section{Cite this: DOI: $10.1039 / \mathrm{c0xx00000x}$}

a)<smiles>C=CCC(=O)O</smiles>

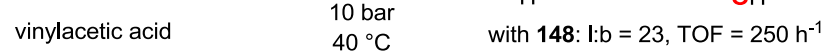
with 149: $\mathrm{l}: \mathrm{b}=41$, TOF $=350 \mathrm{~h}^{-1}$<smiles>C=CCCCC(C=C)C(=O)O</smiles>

150

b)<smiles>[R]C=CC(=O)O</smiles>

$\alpha, \beta$-unsaturated acids

$\left[\mathrm{Rh}(\mathrm{acac})(\mathrm{CO})_{2}\right](0.67 \mathrm{~mol} \%)$

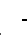

$148(6.7 \mathrm{~mol} \%)$

$\mathrm{CO} / \mathrm{H}_{2} 1$

$25^{\circ} \mathrm{C}$

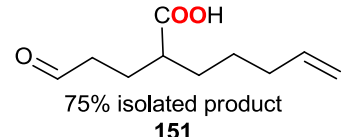
151

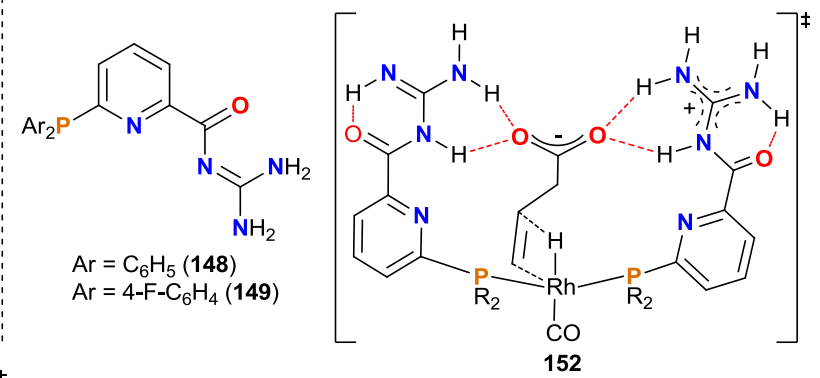

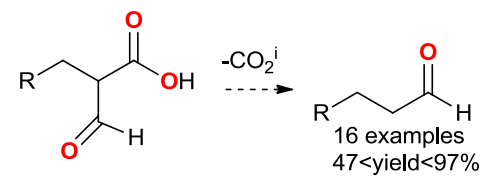

$25^{\circ} \mathrm{C}$

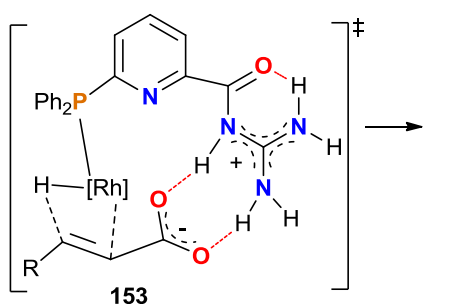

c)

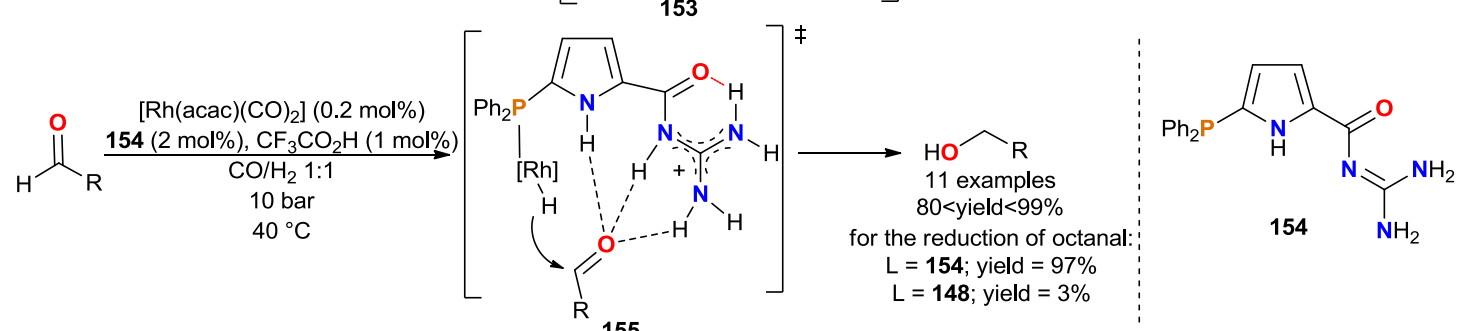

d)

$\left[\mathrm{Rh}(\mathrm{acac})(\mathrm{CO})_{2}\right](0.5 \mathrm{~mol} \%)$

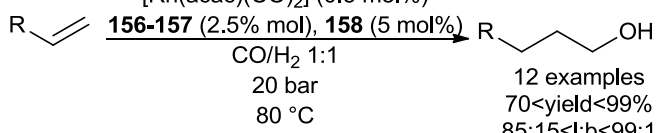

$70<$ yield $<99 \%$
$85: 15<1: b<99: 1$

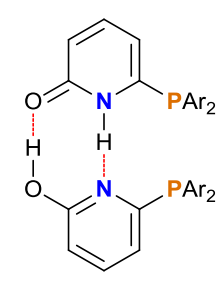

156-157<smiles>COc1ccc(P(c2ccc(OC)cc2)c2ccc(C(=O)N=C(N)N)[nH]2)cc1</smiles>

158

active for hydroformylation active for hydrogenation

Fig. 37 Bifunctional rhodium catalysts with a remote basic guanidine group. (a) Ligand - substrate interactions between 148 or 149 and vinylacetic acid enable very active and selective hydroformylation. 152 is a possible structure of the selectivity-determining hydrometalation step. (b) Selective hydroformylation of $\alpha, \beta$-unsaturated carboxylic acids with 148. ${ }^{i}$ Decarboxylation may also be rhodium-catalysed. (c) Phosphino- $1 H$-pyrrole-25 acylguanidine ligand $\mathbf{1 5 4}$ catalyses the hydrogenation of aldehydes. (d) Tandem hydroformylation/hydrogenation catalysis with a combination of 156-157 and 158. Refs: see the text.

acids are used as substrates, catalyst derived from the acylguanidine-functionalized phosphine ligand 148 mediated the regioselective formation of $\alpha$-formyl acids which, after 10 decarboxylation, give linear aldehydes (Fig. 37, b). ${ }^{406}$ This time, the carboxylate-guanidinium ion pair directs the Rh-attack at the $\alpha$-position of the carboxylate anion (see 153). The precise nature of the ligand - substrate interaction has not been established in that case, but the complexation of the carboxylate anion by two 15 guanidine moieties, similarly to $\mathbf{1 5 2}$, seems reasonable.

Following the same approach, Breit group applied phosphino$1 H$-pyrrole-2-acylguanidine ligands $\mathbf{1 5 4}$ in the rhodium-catalysed hydrogenation of aldehydes (Fig. 37, c). ${ }^{407}$ In this catalytic reaction, ligand $\mathbf{1 5 4}$ proved to be far more efficient than ligand 20 148. Eleven aldehydes are reduced with yields ranging between 80 and $99 \% . \mathrm{CF}_{3} \mathrm{CO}_{2} \mathrm{H}$ has a positive effect on the catalytic rate probably because it increases the concentration of the guanidinium directing group. The guanidinium cation can also be formed by: i) heterolytic cleavage of hydrogen or, ii) oxidation addition of $\mathrm{H}_{2}$ to 25 the rhodium atom followed by proton transfer to the guanidine moiety. Several hydrogen bonds occur between the guanidinium group of the phosphine ligand and the oxygen atom of the aldehyde. These interactions limit the degree of conformational flexibility of the aldehyde substrate and direct the carbonyl groups 30 towards the $\mathrm{Rh}-\mathrm{H}$ bond. The catalyst provides both an acidic and a hydridic hydrogen atom from the guanidinium moiety and the rhodium centre, respectively (see 155, Fig. 37, c). The pyrrole N$\mathrm{H}$ proton also participates to the binding of the aldehyde, and this interaction may explain why 


\section{Cite this: DOI: 10.1039/c0xx00000x}

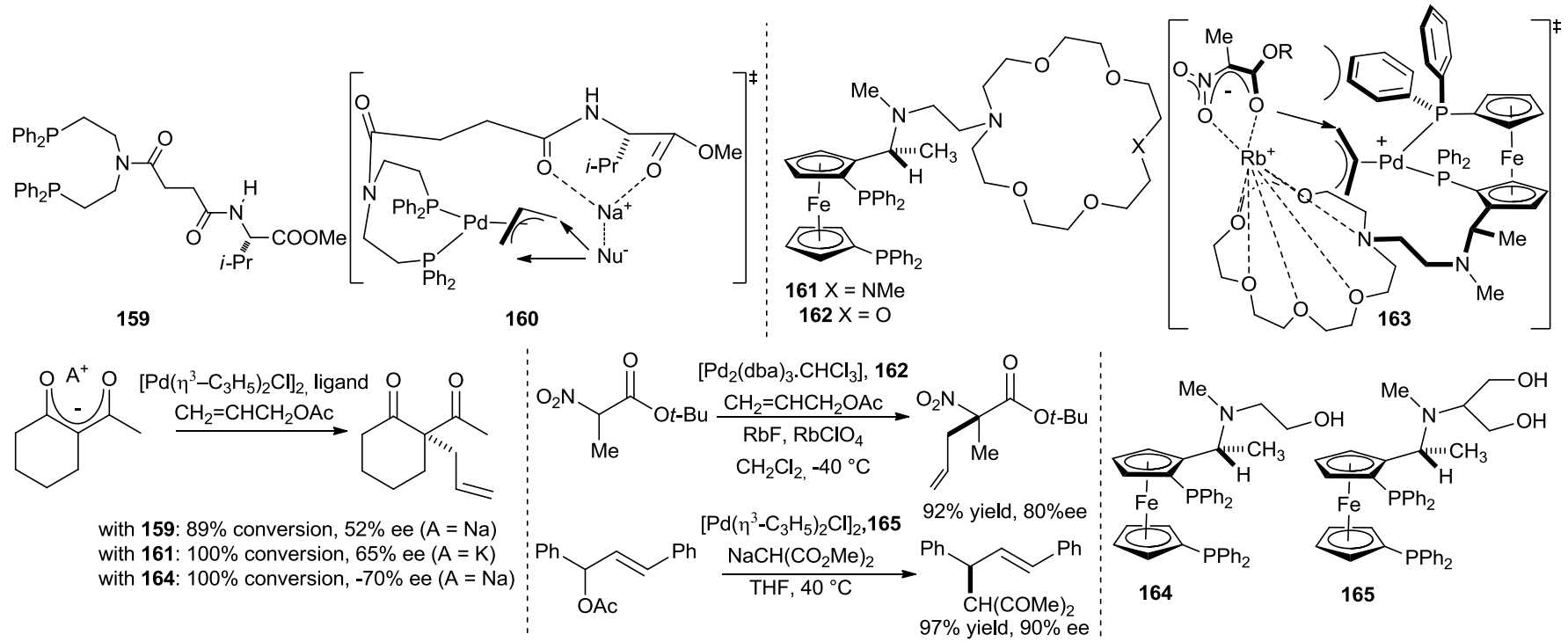

Fig. 38 The selectivity in palladium-catalysed allylation reactions is dictated by reversible ion-dipole $(\mathbf{1 5 9}, \mathbf{1 6 1}, \mathbf{1 6 2})$ or hydrogen bond interactions $(\mathbf{1 6 4}$ and 165) established between a terminal group of the ligand and the substrate. Refs: see the text.

ligand $\mathbf{1 5 4}$ is superior to $\mathbf{1 4 8}$ for the reaction. Interestingly, a 5 syngas atmosphere is required for the hydrogenation and the catalytic resting state is probably $\left[\mathrm{Rh}(\mathrm{H})(\mathrm{CO})_{2}\left(\mathrm{PR}_{3}\right)_{2}\right]$, the usual trigonal bipyramidal hydroformylation resting state. Accordingly, ligand $\mathbf{1 5 4}$ is also active for the tandem hydroformylation/hydrogenation of alkenes. However, a 10 combination of the self-assembled phosphine ligand 156-157, (for hydroformylation), of the phosphino- $1 H$-pyrrole-2-acylguanidine ligand 158 (for hydrogenation) and of $\left[\mathrm{Rh}(\mathrm{acac})(\mathrm{CO})_{2}\right]$ is more efficient for the tandem hydroformylation/hydrogenation of $n$ olefins yielding the respective linear alcohols in high yields (Fig. 15 37, d). ${ }^{113}$ HP-IR studies reveal that at least three different catalytic resting states are in equilibrium in the media; $[\mathrm{Rh}(\mathrm{H})(\mathbf{1 5 6}$ 157)(CO)2], which contains the self-assembled ligand $\mathbf{1 5 6 - 1 5 7}$, is responsible for the high hydroformylation activity of the tandem catalytic system.

20 5.1.2.2 Metal ligands with tethered ionic or hydrogen bond donor/acceptor groups

The incorporation of ionic or hydrogen bond donor/acceptor groups in the backbone of catalysts enables dynamic interactions between the ligand and the substrate or the reaction intermediate 25 and no proton exchange between these reaction partners is suspected.

More than 30 years ago, Kumada and co-workers examined whether the selectivity in palladium-catalysed asymmetric allylation reactions of various nucleophiles can be improved by 30 means of reversible ligand - substrate interactions. With diphosphine ligand 159, the allylic product of the reaction between the enolate anion of 2-acetylcyclohexanone and allylic acetate is obtained with $52 \%$ ee at $89 \%$ conversion (Fig. 38). ${ }^{408}$ Because a derivative of $\mathbf{1 5 9}$ lacking the terminal ester group is less selective, 35 the authors proposed that the chelation of the sodium countercation of the enolate by the ester group of $\mathbf{1 5 9}$ (ion-dipole interactions) is required and controls the stereochemical outcome of the reaction (see 160, Fig. 38). The enantioselectivity of the allylic product is modest but yet quite remarkable considering that the chiral centre 40 is located 10 atoms away from the catalytic metal centre. Iondipole interactions between remote groups of ligands and enolate substrates were further explored by Ito, Sawamura and co-workers. A series of chiral ferrocenylphosphine derivatives appended with crown ether moieties were evaluated in the same palladium45 catalysed allylic reactions. A combination of $\mathbf{1 6 1}$ and KF proved to be slightly more efficient than $\mathbf{1 5 9}$ providing the allylic product in $65 \%$ ee at full conversion (Fig. 38). ${ }^{409,410}$ Ligand 162 in combination with $\mathrm{Rb}$ salts $\left(\mathrm{RbCl}\right.$ and $\left.\mathrm{RbClO}_{4}\right)$ is selective for the allylation of $\alpha$-nitro ketones (up to $80 \%$ ee). ${ }^{410}$ The nature of: i) the 50 linker between the $\mathrm{Cp}$ ring of the ferrocenylphosphine ligand and the crown ether, ii) the crown ether and, iii) the countercation of the enolate substrate all influence the catalytic performance. The formation of a well-defined ternary complex involving the crown ether, the $\mathrm{Rb}$ or $\mathrm{K}$ countercation of the enolate and the enolate is 55 thought to be at the origin of the high rate and selectivity observed with ligands 161 and $\mathbf{1 6 2}$. The complementarities between the ring size of the crown ether and the size of the $\mathrm{Rb}$ cation likely explain why the combination of $\mathbf{1 6 2}$ and $\mathrm{Rb}^{+}$outperforms the other ligand/enolate countercation combinations. A model that accounts 60 for the observed selectivity was proposed: the steric repulsion between the enolate anion bound to the $\mathrm{Rb}$ cation and a phenyl group of the diphosphine ligand dictates the face of attack of the enolate to the terminal carbon of the $\eta^{3}$-allyl electrophile (see 163, Fig. 38). Hydrogen bond interactions established between the 65 terminal hydroxyl groups of ferrocenylphosphine ligands 164 and 165 and the 


\section{Cite this: DOI: 10.1039/c0xx00000x}

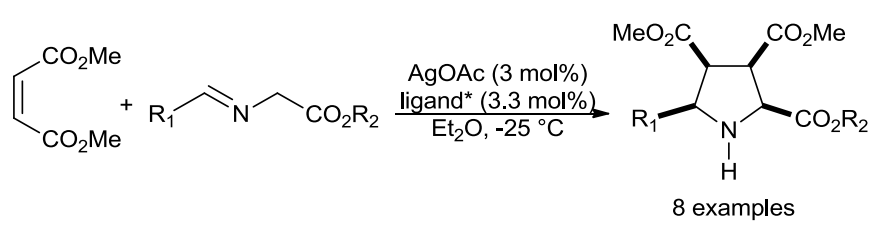

with 166: $89<$ yield $<98 \%,-78<$ ee<- $92 \%$ with 167: $90<$ yield $<98 \%, 36<$ ee $<97 \%$
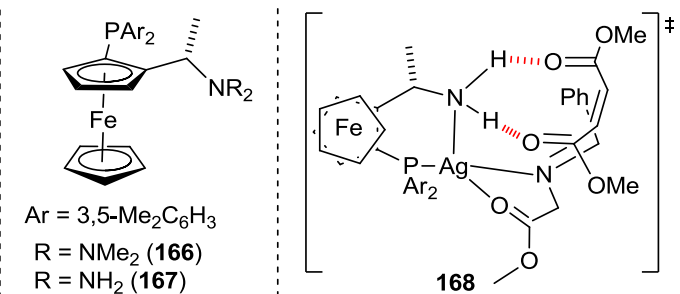

Fig. 39 Hydrogen bond interactions direct the formation of opposite enantiomers for the silver-catalysed asymmetric [3+2] cycloaddition of azomethine ylides. Ref: see the text.

substrate also seem to dictate the stereochemical outcome of 5 palladium-catalysed asymmetric allylation reactions. ${ }^{411-414}$ Interestingly, ligands 164 and 161 exhibited opposite senses of enantioselection for the allylation of 2-acetylcyclohexanone. ${ }^{412}$ At that time, these results were of interest both for their innovative design of ligand - substrate interaction and for the good to 10 excellent selectivity obtained for these challenging asymmetric allylation reactions. Even though more efficient catalysts are now known for this reaction, ${ }^{415}$ these studies have paved the way towards the investigation of bifunctional catalysts for various catalytic applications.

15 In 2008, Zeng et al. found that the ferrocenylphosphine ligands 166 and 167 yielded opposite enantiomers in the silver-catalysed [3+2] cycloaddition of azomethine ylides with dimethyl maleate (Fig. 39). ${ }^{416}$ NMR experiments indicated that the $\mathrm{N}-\mathrm{H}$ proton of 167 interacts with the carbonyl oxygen of the incoming dimethyl 20 maleate substrate. This interaction favours the attack on the top face of the silver-bound azomethine ylide (see 168, Fig. 39). Additionally, hydrogen bond interactions may stabilise the possible zwitterionic intermediate and the transition state. The dimethylamino group of ligand $\mathbf{1 6 6}$ cannot form hydrogen bonds 25 and steric repulsion between the $\mathrm{NMe}_{2}$ group and dimethyl maleate causes the attack on the bottom face of the ylide. Here, the ligand - substrate interaction does not affect the efficiency of the catalyst but directs reversal of enantioselectivity.

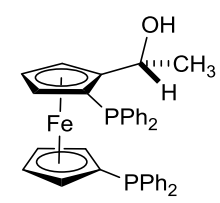

169
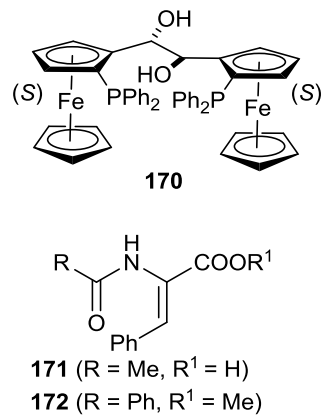

Fig. 40 Rhodium-catalysed asymmetric hydrogenation with phosphine ligands that possess one or two remote hydroxyl groups. 170 has planar chirality $((S, S)$-ferrocene moieties) and central chirality (ferrocene linker). Refs: see the text.
35 Hydrogen bond interactions between substrate and remote parts of ligands can also influence the stereochemical outcome of rhodium-catalysed asymmetric hydrogenation reactions. Notably, phosphine ligands with hydroxyl functional groups attached to their backbone have been studied. ${ }^{417}$ Ferrocenylphosphine ligand 40169 (Fig. 40) hydrogenates pyruvic acid ${ }^{418}$ and 1-phenylvinyl diphenylphosphinate ${ }^{419}$ with $80 \%$ and $78 \%$ ee respectively. Hydrogen bonds between the hydroxyl group of 169 and the $\mathrm{C}=\mathrm{O}$ or $\mathrm{P}=\mathrm{O}$ oxygens of the substrates may dictate the selectivity of the catalytic reaction. Kagan and co-workers found that diphosphine 45 ligand 170, having a chiral diol linker between the two ferrocenylphosphine units, provided the hydrogenation product of 171 with high optical purity $(85 \%$ ee $) .{ }^{420}$ Acetalization of the hydroxyl groups led to a ligand that was far less selective for the same reaction $(45 \%$ ee). However, the positive effect cannot be 50 undoubtedly ascribed to hydrogen bond interactions between the alcohol functions of the ligand and the $\mathrm{C}=\mathrm{O}$ oxygen of the substrate. A positive effect on selectivity was also found by using the phospholane derivative $\mathbf{1 7 3}$ instead of its methoxy analogue 174, e.g. substrate 172 is hydrogenated with $90 \%$ ee $(77 \%$ ee for 55 174). ${ }^{421}$ Hydrogen-bonded ligands (section 3.1.2) can also interact with the substrate during the course of asymmetric hydrogenation reactions. The carbonyl oxygen of the LEUPhos ligand interacts with the hydroxyl group of the substrate during the transition state of the hydrogenation reaction (see the energy-minimized structure 60 of Fig. 3). ${ }^{103}$ PhthalaPhos ${ }^{134,135}$ and Benzaphos ${ }^{142}$ are families of hydrogen-bonded ligands that lose the ligand - ligand interaction at the expense of ligand - substrate interaction along the catalytic cycle (see the transition state structure represented in Fig. 10). Also, in the example provided by Reek and co-workers, the anion ${ }_{65}$ used as chiral inducer forms one hydrogen bond with the substrate (see the molecular model in Fig. 29). ${ }^{284}$ The ability of urea moieties to bind and direct the substrate during the course of the catalytic reaction has also been mentioned in section 3.1 (see Fig. 20). ${ }^{73}$

The structure of a palladium hetero-complex, containing two ${ }_{70}$ Breit ligands based on the 2-amidopyrine and isoquinolone complementary groups (section 3.1.2), was established by X-ray diffraction analysis. The complementary isoquinolone and amidopyridine groups appended to cis-positioned phosphine ligands are linked through a single hydrogen bond (see 175, Fig. 75 41). In addition, the $\mathrm{N}-\mathrm{H}$ proton of the isoquinolone moiety is 

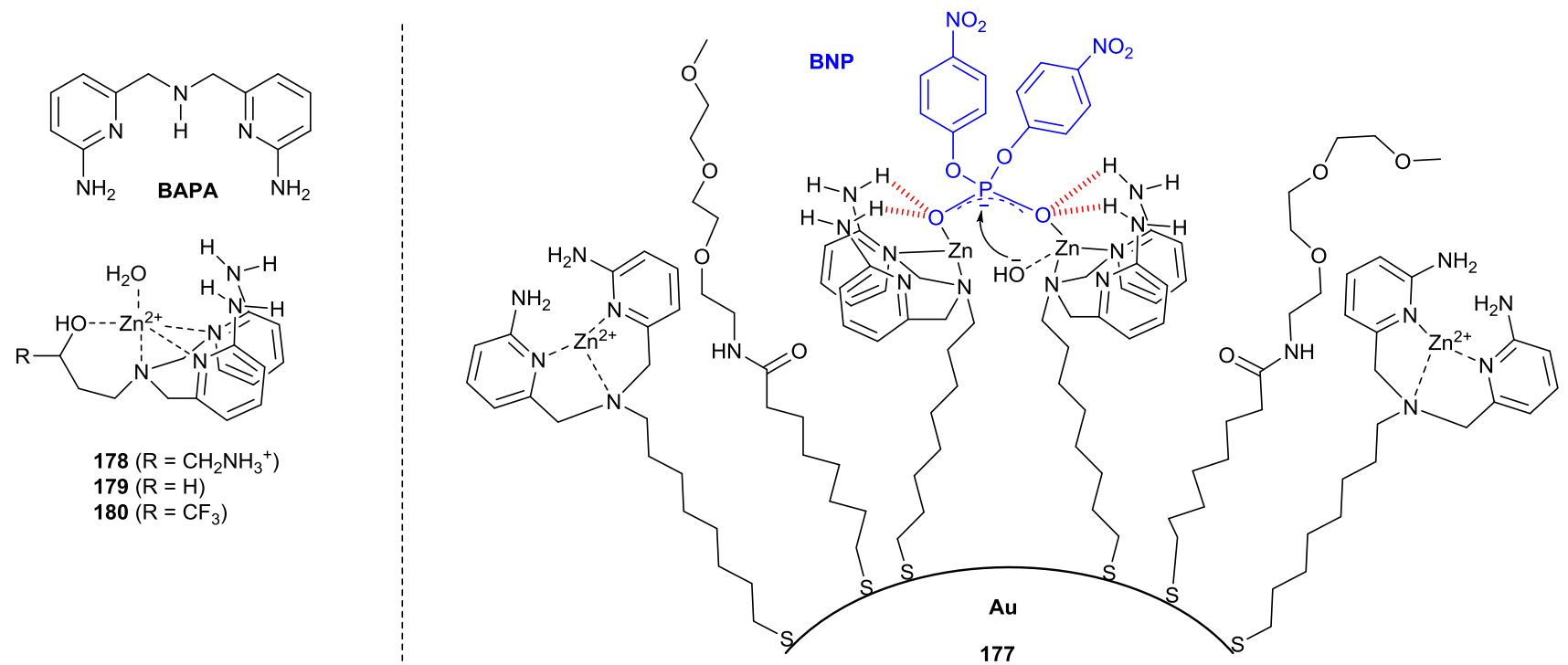

Fig. 42 Nuclease mimics that incorporate pendant amino and ammonium groups. BAPA = bis(2-amino-pyridinyl-6-methyl)amine. 177 is a very active catalyst for the cleavage of BNP and double strand DNA. The mode of activation of BNP by a dinuclear site of $\mathbf{1 7 7}$ is represented on the right. The role of the pendant ammonium group of $\mathbf{1 7 8}$ for the cleavage of phosphate ester bonds has been established. Refs: see the text.

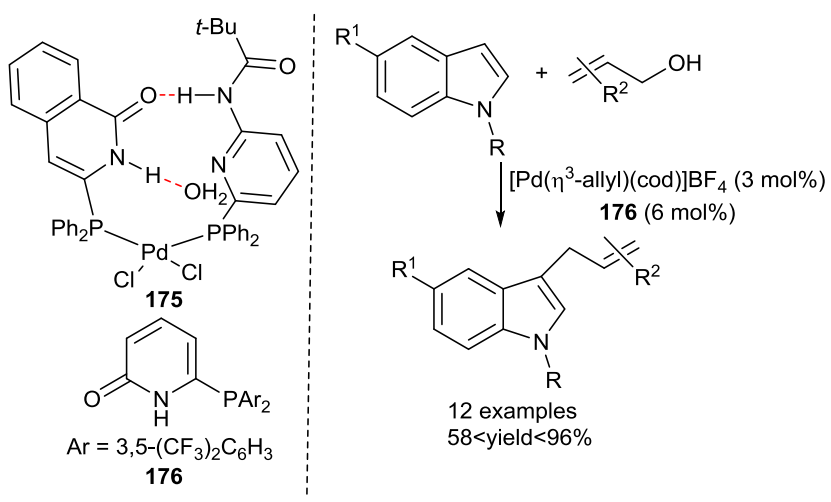

Fig. 41 Hydrogen bond between the $\mathrm{N}-\mathrm{H}$ proton of the isoquinolone moiety of the phosphine coordinated to the Pd and the incoming allylic alcohol likely facilitates the allylation of indole derivatives. Ref: see the text.

10 bound to a water molecule that was present in the recrystallisation process. This structure inspired Breit and co-workers to investigate related palladium catalysts for the allylation of indole derivatives using allylic alcohol derivatives as the allyl source. ${ }^{123}$ Indeed, a combination of $\mathbf{1 7 6}$ and $\left[\mathrm{Pd}\left(\eta^{3}-\right.\right.$ allyl $\left.)(\operatorname{cod})\right] \mathrm{BF}_{4}$ provided the 15 respective allylated indoles with good to excellent yields. Hydrogen bond interactions between the hydroxyl group of the incoming allylic alcohol and the $\mathrm{N}-\mathrm{H}$ proton of the isoquinolone moiety probably make the hydroxyl a better leaving group and favour the formation of the $\eta^{3}$-allyl palladium intermediate.

20 The proximity of one or two acidic centres $\left(\mathrm{Zn}^{2+},{ }^{422-426} \mathrm{Cu}^{2+427}\right.$ or $\mathrm{Co}^{3+428}$ ) and amino groups is important to achieve efficient nuclease mimics. ${ }^{427}$ Copper and zinc complexes of BAPA derivatives (Fig. 42) activate phosphate diesters by the cooperative effect of acidic centres and hydrogen bond interactions. ${ }^{429}$ Bonomi 25 et al. showed that zinc catalysts, supported on Au nanoparticles are very active for the cleavage of bis- $p$-nitrophenyl phosphate (BNP) and also present the unprecedented ability to perform double strand cleavage of DNA. ${ }^{430}$ Both mononuclear and dinuclear sites of the nanoparticle-supported catalyst 177 (Fig. 42) are more active than 30 mono- and binuclear zinc complexes taken as references. The enhanced activity is the result of the very high binding affinity of the phosphate diesters with the $\mathrm{Zn}^{2+}$ cationic catalytic sites located at the surface of the Au-nanoparticles. Ammonium ${ }^{431-435}$ or guanidinium $^{436,437}$ groups were also placed at a distal position of 35 the acidic centre of nuclease mimics but the exact role of these groups during the cleavage of phosphate esters remains to be elucidated. ${ }^{436,438}$ Mancin and co-workers dissect the contribution of the pendant ammonium group of 178 (Fig. 42) in the hydrolytic cleavage of three different substrates. ${ }^{439}$ The activity of the $\mathrm{Zn}$ 40 catalyst 178 was compared with the one obtained with catalyst 179 (that lacks the ammonium group) and catalyst 180 (that possess a $\mathrm{CF}_{3}$ group of similar electronegativity compared to $\mathrm{NH}_{3}^{+}$). Accordingly, they established that, due to its hydrogen bond donor ability, the ammonium group favours the formation of the 45 hydroxide/alkoxyde nucleophile but decreases its reactivity by lowering its intrinsic basicity. Also, the ammonium group stabilises the developing negative charge in the transition state via electrostatic and/or hydrogen bond interactions. Taken together, these results help to understand the complex role played by 50 ammonium groups in nucleases and nuclease mimics. 


\section{Cite this: DOI: $10.1039 / \mathrm{c0xx00000x}$}

\section{www.rsc.org/xxXXXX}

ARTICLE TYPE
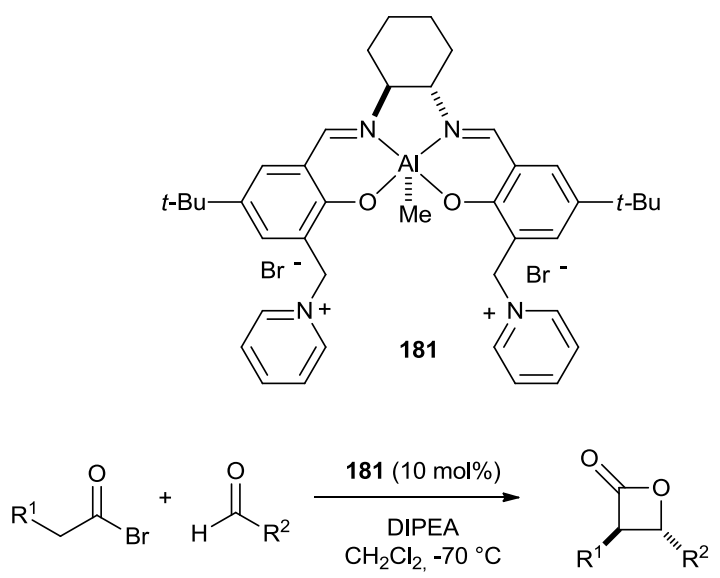

18 examples $62<$ yield $<96 \%$ 87:13<trans/cis< $98: 2$ $70<$ ee $<95 \%$

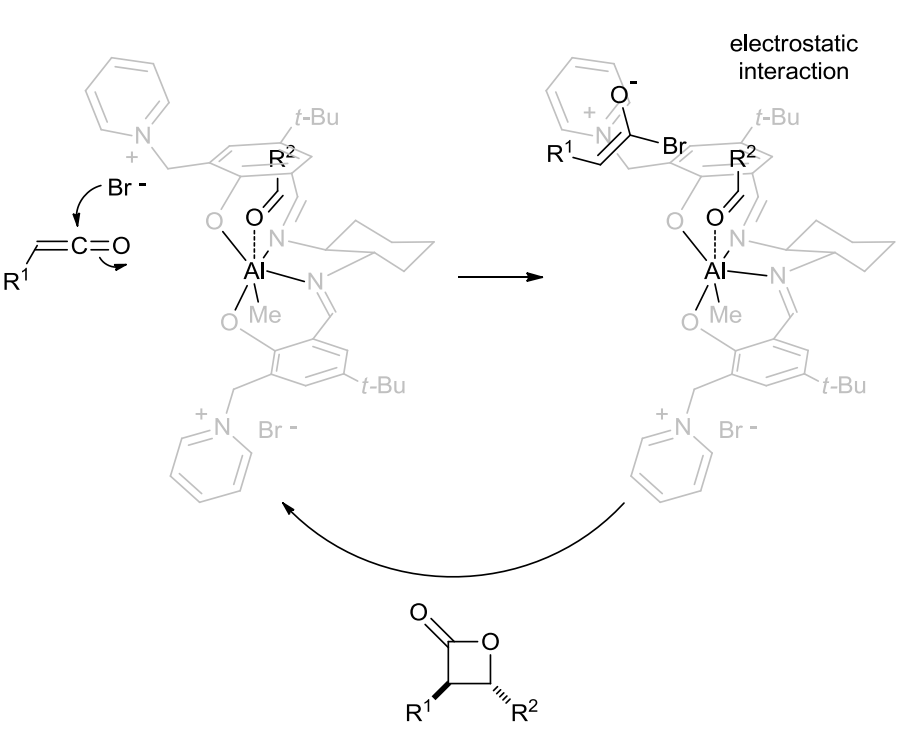

Fig. 43 Complex 181 is a very active and selective catalyst for the asymmetric synthesis of trans-configured $\beta$-lactones. Proposed mechanism for the ion pair-directed Lewis acid catalyst. Ref: see the text.

An unusual example of ion pair-directed Lewis acid catalysis 5 was provided recently. Peters et al. demonstrated that the salen$\mathrm{Al}^{\mathrm{III}}$ complex 181 (Fig. 43), which possess two peripheral pyridinium groups, is a very selective catalyst for the asymmetric $[2+2]$ cyclocondensation of acyl bromides, as ketene precursors, and aldehydes. ${ }^{440}$ This catalyst is far more active and selective 10 towards the formation of the trans-configured $\beta$-lactones than related salen- $\mathrm{Al}{ }^{\mathrm{III}}$ complexes bearing different types of tertiary amino groups. The E-configured enolate is formed in situ by reaction between the ketene and the bromine counteranion of one pyridinium group of $\mathbf{1 8 1}$. This enolate forms an ion pair with one 15 peripheral pyridinium group of the ligand and this interaction likely dictates the stereochemical outcome of the reaction. In the proposed mechanism, the ion pair stabilizes the reactive enolate that is now in a suitable position to undergo the [2+2] cyclocondensation with the Al-coordinated aldehyde.

20 Crabtree and co-workers were probably the first who used molecular modelling as a tool to probe the geometry of hydrogenbonded ligand - substrate complexes. ${ }^{441-444}$ They designed a terpyridine ligand decorated with a peripheral $\mathrm{COOH}$ derived from Kemp's triacid. This $\mathrm{COOH}$ group is directed towards the upper 25 part of the terpyridine plan at a suitable position for substrate recognition. The dinuclear $\mathrm{Mn}$ complex of this ligand was evaluated as a catalyst for the oxidation of ibuprofen and other substrates which contain a $\mathrm{COOH}$ moiety (see 182, Fig. 44, a). Within the hydrogen-bonded ligand - substrate assembly, the 30 benzylic $\mathrm{C}-\mathrm{H}$ bond of ibuprofen is located in close proximity to the $\mathrm{M}=\mathrm{O}$ group. As expected, this bond is preferentially oxidized compared to the tertiary $\mathrm{C}-\mathrm{H}$ bond of the $\mathrm{CH}-\mathrm{COOH}$ group (TON =580; selectivity for the benzylic over tertiary oxidized $\mathrm{C}-\mathrm{H}$ bond
$=66: 1)$. Oxidation of a mixture of cis- and trans 435 methylcyclohexyl acetic acid $\mathbf{1 8 3}$ takes place in a diastereoselective way at its remote tertiary $\mathrm{C}-\mathrm{H}$ bond; the oxidation product 184 with the hydroxyl and carboxymethyl substituents in a relative trans-arrangement is the only observed one (Fig. 44, b). ${ }^{443}$ The presence of two recognition units in 40 catalyst 182 makes possible the formation of two hydrogen-bonded ligand - substrate complexes which can act together to block both faces of the catalyst at once. These hydrogen-bonded ligand substrate assemblies sterically prevent free substrates to reach the active site where they would react unselectively. The two hydrogen ${ }_{45}$ bonds established between the $\mathrm{COOH}$ groups of the catalyst and the substrate are maintained during the whole catalytic pathway and the selective $\mathrm{C}-\mathrm{H}$ oxidation occurs at lower energy than without molecular recognition. Adequate substrates possess a complementary $\mathrm{COOH}$ group and an appropriate distance between ${ }_{50}$ the acid-group and the targeted $\mathrm{C}-\mathrm{H}$ bond. The same authors compared the catalytic performance of $\mathbf{1 8 2}$ and a mononuclear $\mathrm{Mn}^{\mathrm{III}}$-porphyrin complex appended with four peripheral $\mathrm{COOH}$ groups towards the epoxidation of cycloalkene acetic acid substrates. $^{445}$ Dinuclear complex $\mathbf{1 8 2}$ provided the respective 55 epoxides in good to excellent yields whereas the mononuclear $\mathrm{Mn}^{\mathrm{III}}$-porphyrin complex was poorly active. The diastereoselectivity is rather low for these epoxidation catalysts as a probable result of different possible orientations of the substrate in the catalytic pocket.

60 Probably inspired by the work of Crabtree and co-workers, several catalysts have been designed to orient the substrate through hydrogen bond interactions so that remote oxidation of a double bond or a sulfur atom occurs with high selectivity. Mirkin 


\section{Cite this: DOI: 10.1039/c0xx00000x}

(a)
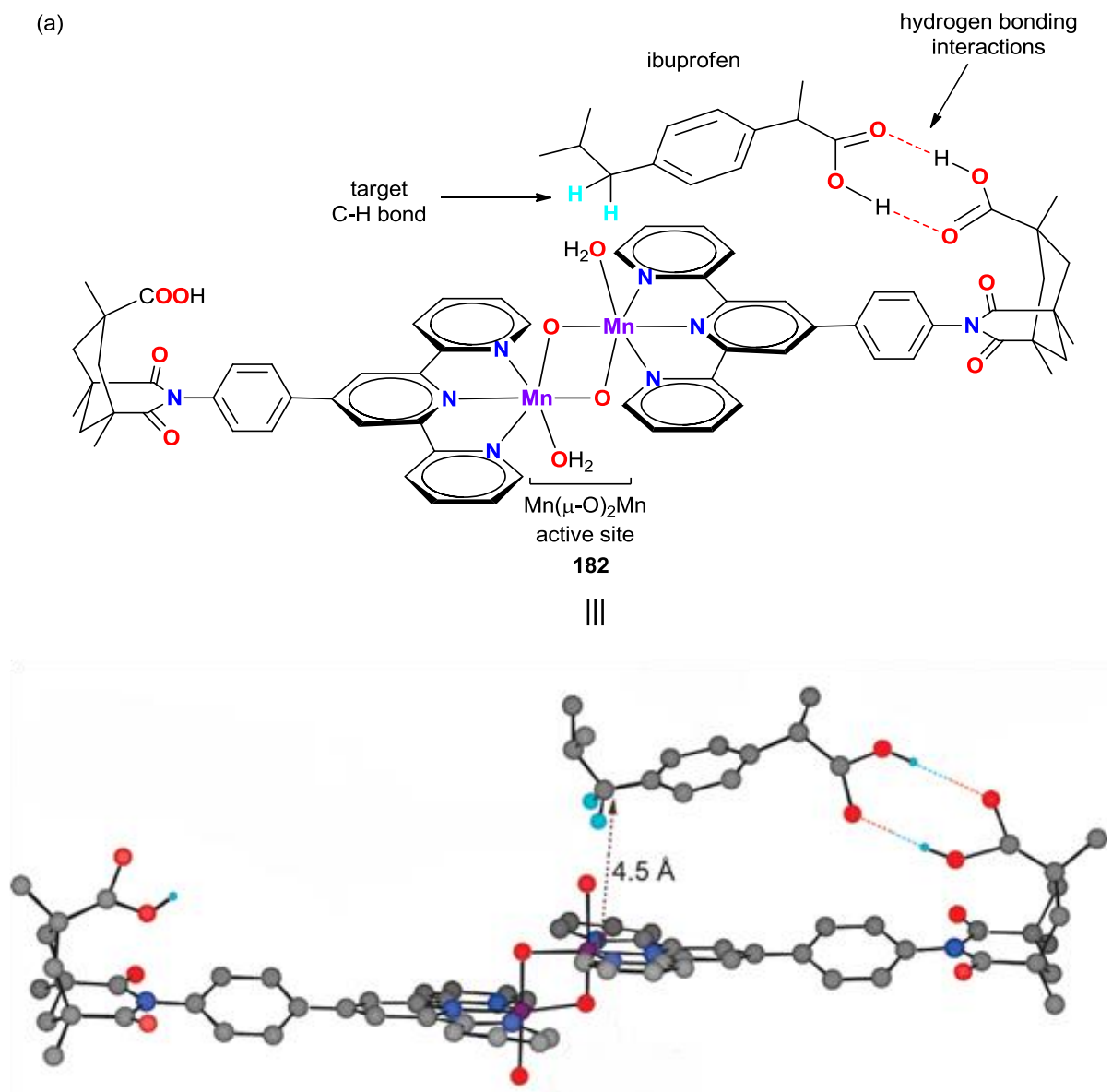

(b)

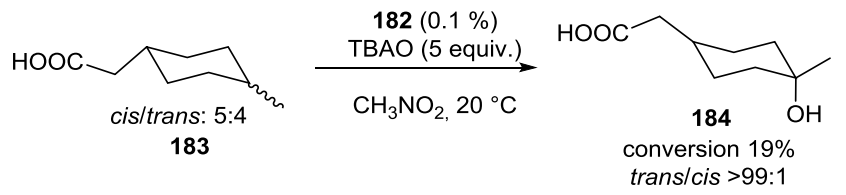

Fig. 44 Well-designed hydrogen-bonded ligand - substrate complexes. (a) Catalyst derived from 182 selectively oxidises the benzylic C-H bond of 5 ibuprofen. Molecular model of the complex. The distance between the target $\mathrm{C}$-atom and the manganese atom is $\mathrm{ca}$. $4.5 \AA$. (The molecular model is reprinted with permission from ref. 443. Copyright 2008. American Chemical Society). (b) Diastereoselective oxidation of $\mathbf{1 8 3}$ with $\mathbf{1 8 2}$ as precatalyst and $\mathrm{TBAO}$ as oxidant. $\mathrm{TBAO}=$ tetrabutylammonium oxone. Refs: see the text.

and co-workers prepared a dinuclear complex composed of a central chiral Jacobsen-Katsuki $\mathrm{Mn}^{\mathrm{III}}$-salen catalyst appended with 10 an amidopyridine group (see 185, Fig. 45, a). ${ }^{446}$ The formation of a 1:1 complex between the amidopyridine group of $\mathbf{1 8 5}$ and the carboxylic acid of 4-vinylbenzoic acid was confirmed by ITC (K $=3090 \pm 360 \mathrm{~L}^{\mathrm{mol}}{ }^{-1}$ ). Also, computational studies indicate that, within this hydrogen-bonded ligand - substrate complex, the 15 double bond is at an ideal position with respect to the Mn centre to undergo oxidation (3.9 $\AA$ ). In a competition experiment with a 1:1 mixture of 4-vinylbenzoic acid and styrene, catalyst $\mathbf{1 8 5}$ preferentially oxidized the former. Catalyst 185 also yields the opposite enantiomer compared to catalyst $\mathbf{1 8 6}$ lacking the
20 amidopyridine moiety but the enantioselectivity is low in both cases. The substrate selectivity observed with $\mathbf{1 8 5}$ is a result of its ability to form a hydrogen-bonded complex with 4-vinylbenzoic acid. Bach et al. applied the Mn-salen complex 187, with peripheral amide groups derived from the 325 azabicyclo[3.2.1]octan-2-one scaffold, in the enantioselective oxidation of sulfides (Fig. 45, b). ${ }^{447}$ Catalyst derived from 187 oxidized $2 H$-benzo[e][1,4]thiazin-3-one into the respective sulfoxide in $76 \%$ yield and $67 \%$ ee. A series of control experiments confirmed that the hydrogen bond interactions between the remote 30 lactam group of the ligand and the quinolone binding motif of the substrate were solely 


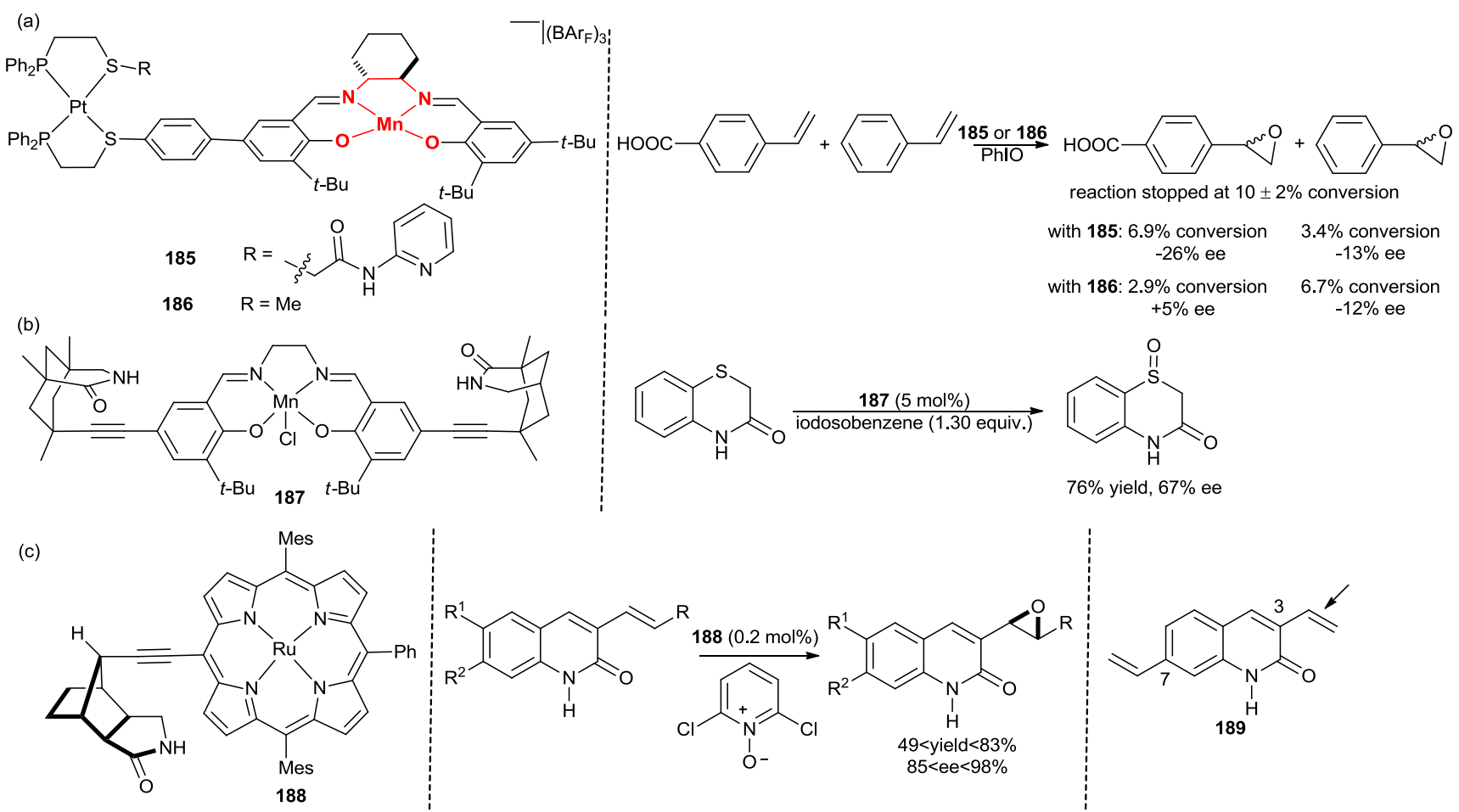

Fig. 45 Salen or porphyrin metal catalysts which possess a peripheral receptor for substrate binding. The respective catalysts were applied: in the epoxidation of 4-vinylbenzoic acid (a), in the oxidation of $2 \mathrm{H}$-benzo[e][1,4]thiazin-3-one (b) and in the epoxidation of 3-alkenylquinolones (c). Refs: see the text.

5 responsible for face discrimination. However, the substrate scope was somewhat limited since substrates with an isoquinolone group were not selectively oxidized. The same group designed the Ruporphyrin complex 188 with an appended chiral octahydro- $1 H$ 4,7-methanoisoindol-1-one skeleton (Fig. 45, c). ${ }^{448}$ Complex 188 10 catalysed the epoxidation of 3-alkenylquinolones with good yields and selectivities employing 1.1 equiv of 2,6-dichloropyridine-Noxide as the oxidant. ${ }^{449}$ Also, the double bond in the 3-position of 189 is oxidized specifically $(88 \%$ ee). In the most favourable transition state, the quinolone function of the substrate interacts 15 with the lactam group of the ligand via a two-point hydrogen bond. This interaction favours a $R e$ face attack of the metal-oxo on the $s$ trans conformer of the substrate. The catalyst exhibits a good degree of flexibility because $\mathrm{C}=\mathrm{C}$ bonds in 3-alkenylpyridone derivatives are also oxidized with high selectivity (up to $88 \%$ ee) 20 whereas the selectivity is only slightly lower with a 3 -alkenylamide derivative $(70 \%$ ee).

In the course of their studies on the cobalt-catalysed asymmetric cyclopropanation of electron-poor olefins, Zhang and co-workers found a remarkable ligand effect. ${ }^{450-454}$ Porphyrin ligands with 25 peripheral amide groups, such as 190 (Fig. 46), usually displayed higher activity than porphyrin ligands lacking the amide functionality. Mechanistic studies established that the $\mathrm{N}-\mathrm{H}$ proton of the ancillary amide group may interact with the carbonyl oxygen of the ester function of methyl diazoacetate

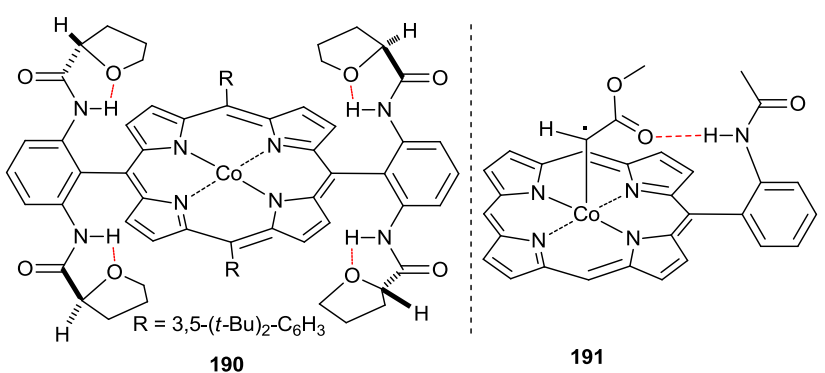

Fig. $46 \mathrm{~N}-\mathrm{H}$ effect observed for the $\mathrm{Co}^{\mathrm{II}}$-catalysed cyclopropanation of olefins. Refs: see the text.

substrate and stabilise the formation of the transient 'carbene' species (see 191, Fig. 46). ${ }^{455}$

35 Reek and co-workers applied a diphosphine ligand that contains a neutral anion receptor in its backbone (99, Fig. 29) for the hydroformylation of $\omega$-unsaturated carboxylates and phosphonates. $^{456}$ The rhodium catalyst which contains the diphosphine ligand 99 gave selectively the linear aldehydes (1:b 40 ratio $>40$ ) in the hydroformylation reaction of 4-pentenoate and 3butenylphosphonate anions. With other substrates, for which the distance between the anion and the alkene function does not exactly span the distance between the receptor and the metal centre, the selectivity is far lower. Like for the acylguanidine 


\section{Cite this: DOI: 10.1039/c0xx00000x}
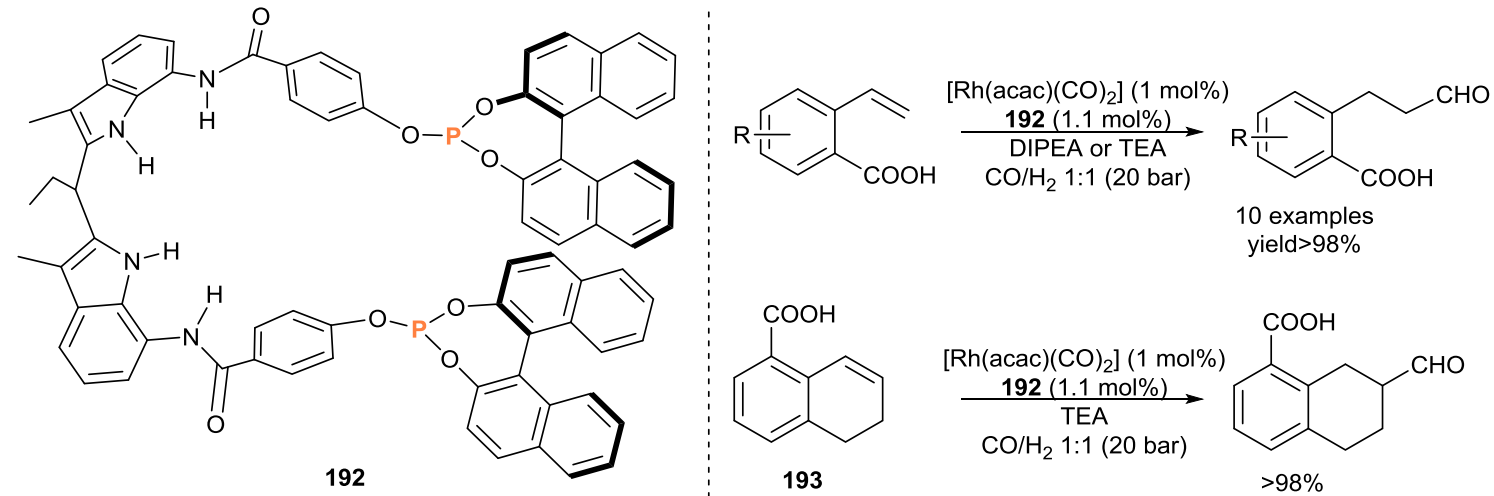

Fig. 47 Designed ligand - substrate interactions for selective hydroformylation reactions. Left: structure of ligand 192 that possesses an anion receptor in its backbone. Rhodium catalyst derived from 192 led quasi exclusively to the formation of linear aldehydes as the hydroformylation products of 2-vinyl benzoic derivatives as well as $\beta$-substituted vinyl arenes such as 193. The hydroformylation product of 193 is racemic. Ref: see the text.

s phosphine ligands (see 148 and $\mathbf{1 4 9}$, Fig. 37), the high selectivity and activity is explained by the stabilisation of the transition state during the regioselectivity-determining hydrometalation step. The system was further exploited for the selective hydroformylation of 2-vinyl benzoic acid. ${ }^{457}$ A slightly modified ligand was prepared $10(\mathbf{1 9 2}$, Fig. 47) that led exclusively to the formation of the linear aldehyde under mild reaction conditions. DFT calculations confirmed that within the ligand - substrate assembly, the conformational lability of the double bond is limited. It can only rotate in the direction of the hydride migration transition state 15 which leads to the $\beta$-phenylalkyl rhodium complex. The $\pi$-benzyl intermediate that is usually favoured cannot be formed while the carboxylate is bound to the pocket. This catalytic system is also efficient for $\beta$-substituted vinyl arenes that contain an internal double bond (see 193, Fig. 47). This catalytic system clearly 20 demonstrates the utility of well-designed ligand - substrate interactions since no covalent ligands are known to date for this reaction (they usually favour the formation of the branched aldehydes) and the resulting aldehydes are relevant intermediates towards bioactive compounds.

25 The examples described in this section highlight the potential of metal bifunctional catalysts, as highly selective and practical catalytic systems for diverse transformations (stereoselective [2+2] cyclocondensations, $\mathrm{C}-\mathrm{H}$ activations, oxidations, hydrogenations, hydroformylations, and allylic substitutions, amongst others). The 30 high catalyst performances and (stereo)selectivities rely on substrate activation and/or directed orientation of the reactants around the catalytic site by specifically designed secondary interactions between reagents and catalyst. Stabilisation of key catalytic intermediates and selectivity-determining transition states 35 also play a role. The aforementioned catalytic systems are highly selective, but also highly substrate dependent: generality has been sacrificed for substrate specificity. Further development must focus on the design of bi- or multifunctional catalysts that can transform complex substrates into highly valuable products.

\section{5.2 Supramolecular organocatalysis}

\subsubsection{Class of catalysts}

Organocatalysis, the use of small organic molecules as catalysts either in solution or in the solid state, ${ }^{458-461}$ has witnessed an intense resurgence since the year 2000 following the two seminal ${ }_{45}$ discoveries reported independently by List and Barbas ${ }^{462}$ (prolinebased enamine catalysis) and by MacMillan ${ }^{463}$ (imidazolidinonebased iminium catalysis). In addition to the reversible covalent mode of activation exhibited by the organocatalysts containing an amino group, ${ }^{464} 468$ which were referred in the paragraph above, so many other organocatalytic systems have been developed that interact with the substrate(s)/reaction intermediate(s) through reversible non-covalent interactions. Surprisingly, although the reaction mechanisms that are proposed for the organocatalysed processes involve many reversible non-covalent interactions, 55 organocatalysts are seldom considered as functional examples of supramolecular catalysts. ${ }^{69,469,470}$ Establishing a clear cut distinction between organocatalysts that activate the substrate by means of reversible covalent or non-covalent interactions is an arduous task which requires an accurate understanding of the ${ }_{60}$ catalytic process, i.e. the nature of the key catalytic intermediates and the transition state. This is also true for the myriad of reported catalytic systems that combine an organocatalyst with a metal, ${ }^{52,53,272,471-475}$ or two organocatalysts ${ }^{476-478}$ working in synergy or in tandem. The fact that structurally similar ${ }_{65}$ organocatalysts operate through quite different reaction mechanisms makes the extraction of general trends for their reactivity a very complex endeavour.

In any case, several types of functional groups are known that may recognize and activate a substrate(s) by means of reversible 70 non-covalent interactions. These functional groups constitute an important element of design of the organocatalysts; they can be embedded in the organocatalyst backbone and mixed with other functional groups (bi- and multifunctional organocatalysts). We 


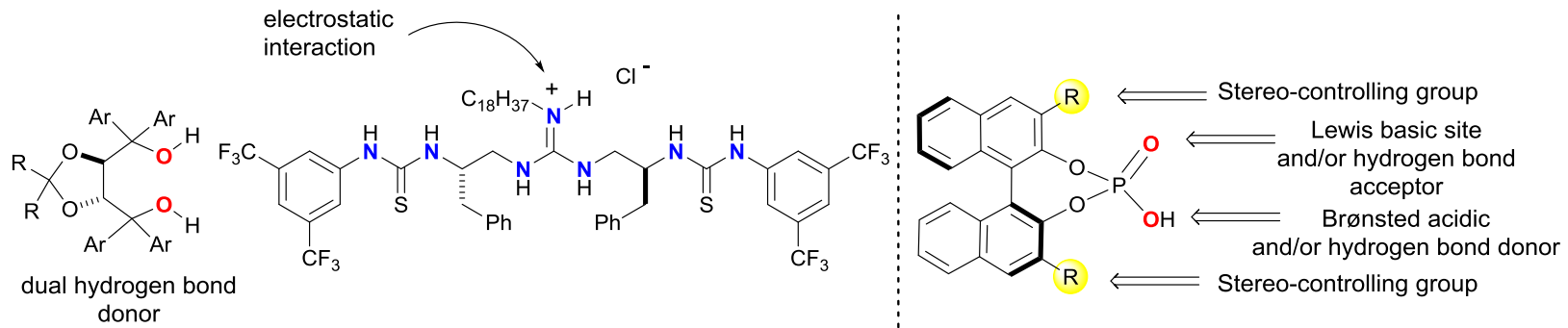

Hydrogen bond donors (A)

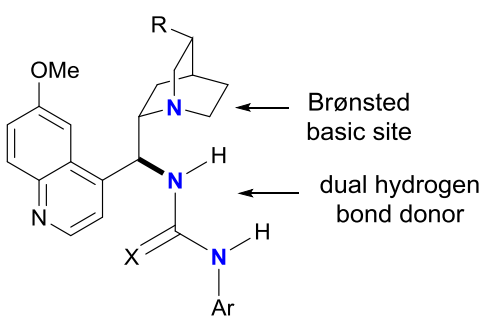

Cinchona alkaloid derivatives (C)

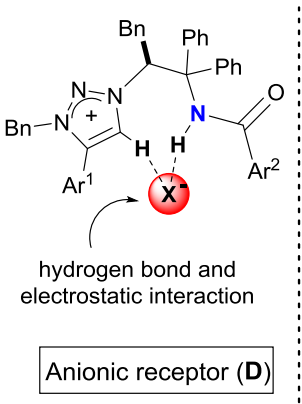

Phosphoric acids (B)
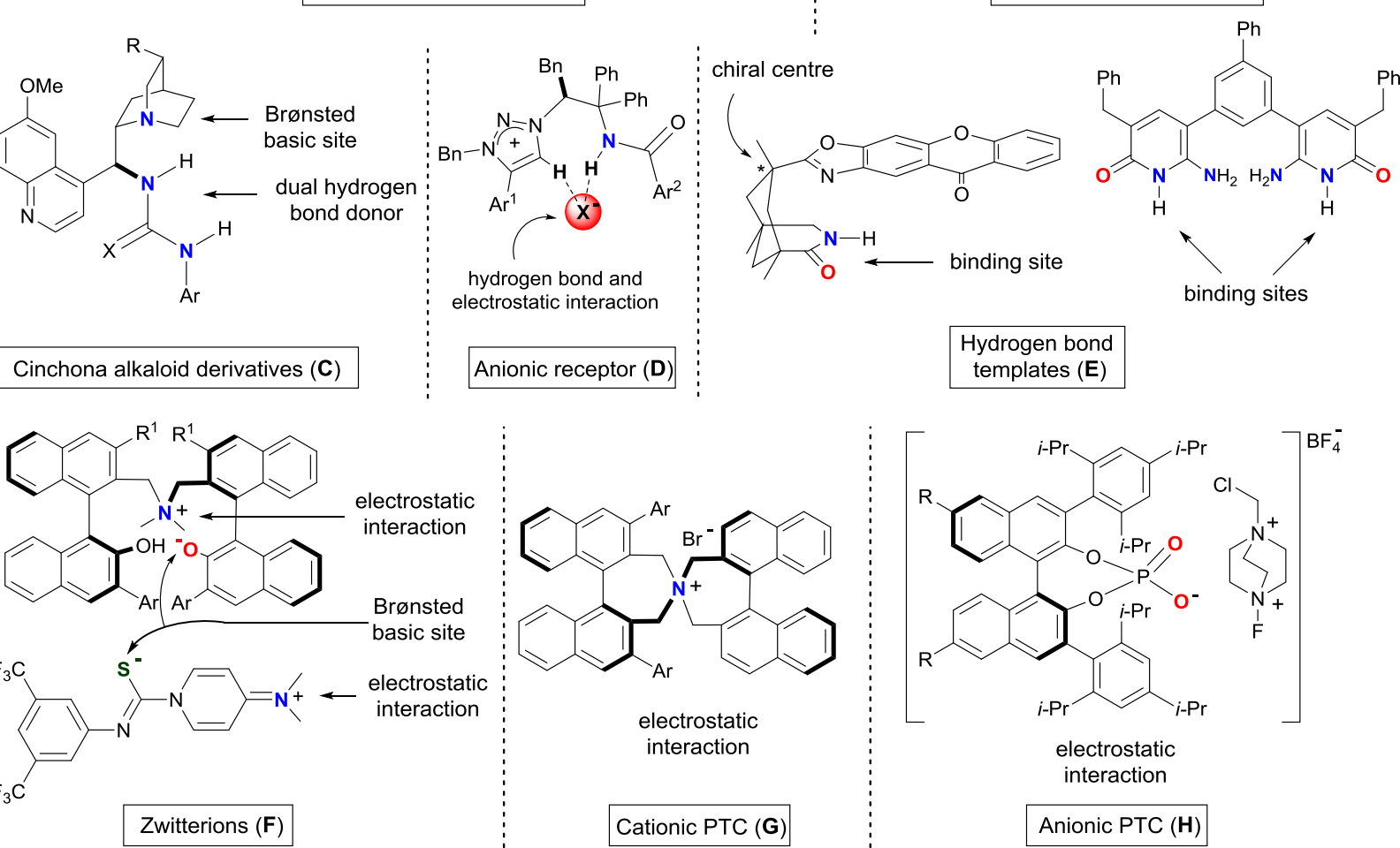

Fig. 48 Classes A-H of widely used supramolecular organocatalysts that possess one or several functional groups for the reversible recognition and activation of substrate(s) and reaction intermediate(s) or the stabilisation of transition states. Refs: see the text.

highlight the following classes of organocatalysts that incorporate 5 one or several of these functional groups (Fig. 48): i) hydrogen bond donor organocatalysts (A,), ${ }^{61,64,68,479-482}$ ii) chiral phosphoric acids as organocatalysts (B), ${ }^{52,53,63,483-487}$ iii) bifunctional organic catalysts which combine a Brønsted basic amino group and a hydrogen donor group in the scaffold of a cinchona alkaloid 10 derivative (C), ${ }^{67,488,489}$ iv) organocatalysts based on anionic receptors (D) $\left.{ }^{490} \mathrm{v}\right)$ molecular templates as organic catalysts that are capable of establishing hydrogen bond interactions with one or two reacting partners (E), ${ }^{491-505}$ vi) zwitterions as organocatalysts (F), ${ }^{506-509}$ vii) cationic chiral phase-transfer organocatalysts 15 (G), ${ }^{65,66}$ and viii) anionic chiral phase-transfer organocatalysts (H). ${ }^{510,511}$ Fig. 48 depicts selected components of each of the highlighted classes of organocatalysts together with the reversible non-covalent interactions associated with their mode of action for substrate(s) activation. As pointed out recently by Jacobsen, non20 easily predictable weak interactions $(\pi-\pi, \mathrm{C}-\mathrm{H}-\pi$, van der Waals, etc...) can play predominant roles in dictating the stereochemical outcome of organocatalytic reactions. ${ }^{4,5}$

In this context, the anion binding ability of chiral ureas and chiral thioureas has been exploited to control the level of 25 selectivity in asymmetric reactions involving the intermediate formation of achiral ion-paired species. ${ }^{481}$ For example, the ion pair 194, consisting in a carboxylate anion and an acyl-pyridinium cation is formed in situ by reacting DMAP (4dimethylaminopyridine) with an acid anhydride. ${ }^{512}$ This achiral ion 30 pair is converted into the three-particle chiral complex 195 upon binding of the carboxylate anion to the chiral thiourea (a, Fig. 49). The supramolecular chiral ion pair $\mathbf{1 9 5}$ is a better acylating agent than the parent achiral acyl-pyridinium salt 194. 


\section{Cite this: DOI: 10.1039/c0xx00000x}

\section{www.rsc.org/xxxxxx}

ARTICLE TYPE
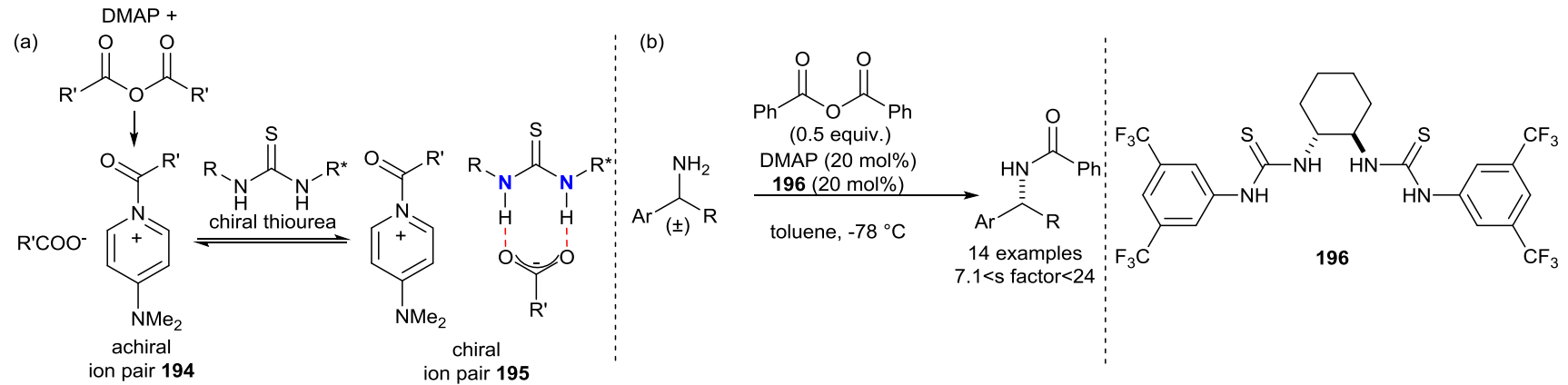

Fig. 49 Example of a catalytic application of the anion-binding strategy. (a) The achiral acyl pyridinium salt is rendered chiral via electrostatic interactions between the carboxylate anion and a chiral thiourea. (b) The combination of DMAP as a nucleophile and of thiourea 196 is efficient for the kinetic resolution of benzylic amines. $s$ factor $=\mathrm{k}_{\mathrm{rel}}=[\ln ((1-\mathrm{C})(1-\mathrm{ee}))] /[\ln ((1-\mathrm{C})(1+\mathrm{ee}))]$ where $\mathrm{C}=$ conversion and ee $=$ enantiomeric excess of the amide product. Ref: see the text.

The binding of the chiral thiourea with the carboxylate anion serves to reduce the electrostatic interaction between this anion and the acyl-pyridinium cation, making the latter more electrophilic. Consequently, DMAP in combination with the chiral thiourea 196 10 formed an efficient catalytic system for the kinetic resolution of benzylic amines ( $s$-factor up to 24, b, Fig. 49). ${ }^{512}$ The nature of the pyridine derivative had a major influence on the stereochemical outcome of the reaction. Thus changing from DMAP to 4-(di- $n$ propyl)amino-pyridine improved the overall catalytic efficiency of ${ }_{15}$ the system. ${ }^{513}$ The same type of chiral three-particle ion pairs proved to be useful for the catalysis of other enantioselective reactions. ${ }^{514-517}$ Ion-pairing catalysis based on this anion-binding strategy has been intensively explored in the last years, ${ }^{5,53}$ and the conceptually-related cation-binding strategy has also been 20 exploited. ${ }^{518,519}$

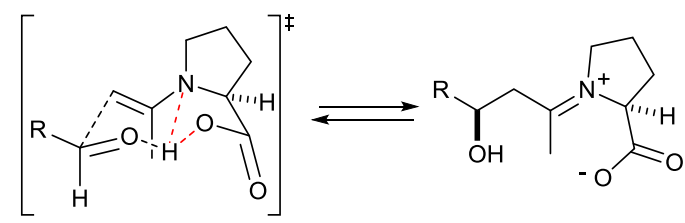

Fig. 50 List-Barbas-Hook transition state for proline-catalysed asymmetric aldol reaction. Refs: see the text

In organocatalysis, the design of bifunctional catalysts is of 25 utmost importance. Bifunctional catalysts do not only bring together the substrates/reaction intermediates, but also they can stabilise the transition state by means of secondary interactions with one of the two sites. Regarding the mechanistic pathway of proline-catalysed asymmetric aldol reactions, ${ }^{462}$ List and Barbas ${ }_{30}$ proposed a metal-free version of the Zimmerman-Traxler transition state (Fig. 50) in which the carboxylic group of the proline is hydrogen-bonded to the oxygen atom of the carbonyl electrophile. Further evidence for this model has been provided by these authors and Hook. ${ }^{520-524}$ The possibility of achieving high 35 rates and selectivities for reactions that involve a well-organised transition state has driven the design of organocatalysts beyond the limits of simple catalyst - substrate interactions. ${ }^{5,525,526}$

\subsubsection{Modularly designed supramolecular organocatalysts}

Taking into account the high number of examples that exist of what 40 can be considered as a "supramolecular organocatalyst" we chose to highlight here only those referring to modularly designed organocatalysts (MDOs).This term was initially proposed by Cong-gui and co-workers ${ }^{527}$ to designate catalytically active supramolecules composed by several molecular building blocks 45 held together by non-covalent interactions. MDOs display analogies to the supramolecular strategies employed in metal catalysis that were described in sections 3,4 and 5.1 of this review. Up to now, MDOs have been designed and applied following two distinct objectives: i) the construction of bifunctional catalysts 50 through the self-assembly of two monofunctional modules and, ii) the modification of the electronic and steric properties as well as the chiral environment of an organocatalyst by means of a complementary additive. The idea here is not to provide a comprehensive overview ${ }^{70}$ of the existing MDOs but rather to ${ }_{55}$ illustrate their applications and the analogies that exist between the strategies used in their construction and those employed in transition-metal supramolecular catalysis.

\subsubsection{Self-assembly of two monofunctional catalysts}

The cooperativity observed between two functional groups in both 60 cinchona alkaloid derivatives (see e.g. C, Fig. 48) and proline (Fig. 50), is very important to achieve highly efficient organocatalysts. In similar manner, the design of supramolecular bifunctional organocatalysts is highly desirable. In doing so, the scope and applications of this new family of organocatalysts will increase. ${ }_{65}$ Likewise, the construction of libraries of supramolecular bifunctional organocatalysts, similarly to the self-assembled ligand strategy mentioned in section 3 , will become a reality.

Cong-gui and co-workers prepared a series of supramolecular bifunctional organocatalysts composed of one aminocatalyst (for 70 nucleophile activation) and a hydrogen bond donor (for electrophile activation) linked to each other by means of ionic 


\section{Cite this: DOI: 10.1039/c0xx00000x}
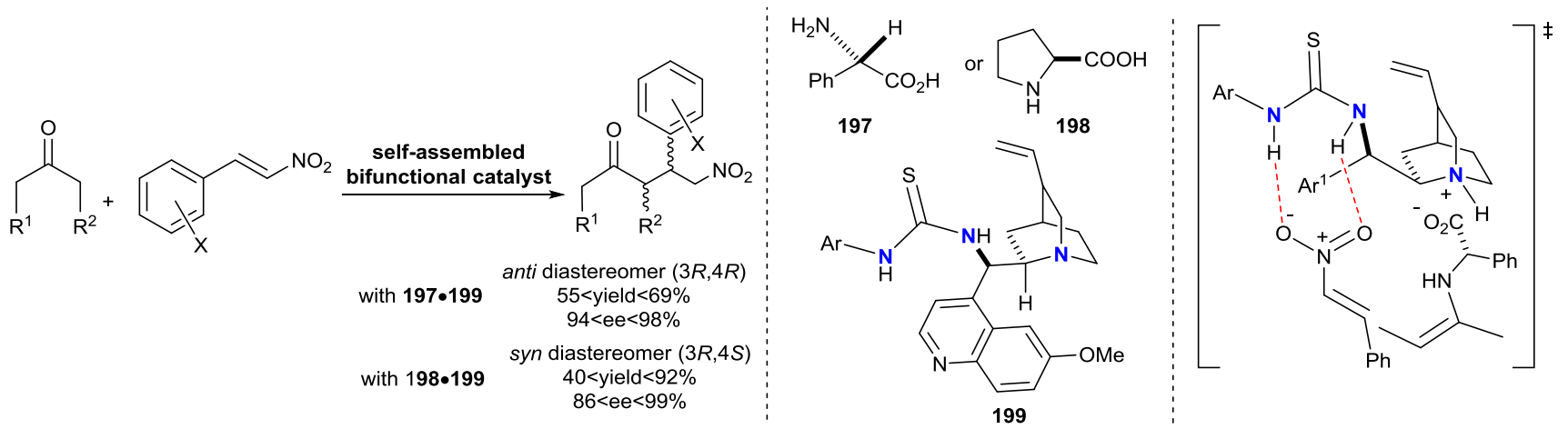

Fig. 51 Self-assembly of two monofunctional catalysts. Application in the asymmetric Michael addition reaction. Right: proposed transition state for the Michael addition of butanone to trans- $\beta$-nitrostyrene involving the ion-pairing catalyst 197•199. Ref: see the text.

hydrogen bonds. Thus, (D)-proline, $(L)$-proline or $(L)$ 5 phenylglycine (containing the amino catalytic centre) were combined with the cinchona alkaloid derivative 199 (the stereocontrolling module) and the resulting MDOs were evaluated for the asymmetric Michael addition of various ketones to $\alpha, \beta$ unsaturated nitro compounds (Fig. 51). ${ }^{527}$ The cinchona alkaloid 10 derivative 199 possesses a tertiary amine group, which is converted into an ammonium group upon protonation by the amino acid. The interaction between the $\alpha$-amino carboxylate anion and the cinchona ammonium cation produced a close-contact ion pair. Whereas low conversion are observed when the modules are used 15 alone for the reaction, high activity and selectivity are obtained with both the $\mathbf{1 9 7} \cdot 199$ and $\mathbf{1 9 8} \cdot 199$ ion pairs. Also, these ion pairs provide opposite senses of enantioselectivity and diastereoselectivity. This observation is rationalized by assuming that in the case of $(L)$-proline the $S i, S i$-attack of the hydrogen20 bonded nitrostyrene on the anti rotamer of the $E$-enamine intermediate is favoured and leads to the formation of the syn diastereomer. Conversely, for $(L)$-phenylglycine, the formation of the $Z$-enamine is favoured and the Re-Si attack of the hydrogenbonded nitrostyrene on this enamine yields the anti diastereomer 25 (see a proposed transition state in the case of $(L)$-phenylglycine in Fig. 51). The same group applied structurally-related supramolecular bifunctional organocatalysts in tandem Michael addition-cyclization reactions ${ }^{528}$ as well as aldol reactions, ${ }^{529}$ however the substrate scope was limited in both cases. An 30 improved substrate scope was obtained by Ramachary et al. in the asymmetric Michael reaction of cyclohexanone with 2-(2nitrovinyl)phenols, also combining proline with a cinchona alkaloid derivative. ${ }^{470}$ The Michael addition reaction afforded hexahydroxanthenol derivatives with high yields, ee and de values.

35 Hexahydroxanthenols are intermediates in the synthesis of medicinally relevant compounds.

The group of $\mathrm{Xu}$ also investigated the self-assembly of two monofunctional catalysts by means of electrostatic interactions. The prepared ion-pairing catalysts display reverse charge 40 distribution compared to the system reported by Cong-gui. ${ }^{530-532}$
That is, proline derivatives are decorated with a side chain containing a Lewis base centre (a pyridine or an imidazole group) that becomes protonated by the carboxylic group of an amino acid or a thioureido acid. The methodologies put forward by the groups 45 of Cong-gui and Xu make possible the screening of a high number of catalytic combinations by simply in situ mixing of the two modules that will be the components of the assembled bifunctional organocatalyst. The observation of matched and mismatched combinations between the two chiral components of the self50 assembled catalyst supports the formation of cooperative catalysts. This class of MDOs constitutes an alternative strategy capable to extend the scope and solve the limitations of the currently employed bifunctional organocatalytic systems.

5.2.2.2 MDOs composed of an organocatalyst and one or several 55 additives

Several MDOs have been developed that combine a functional organocatalyst operating through enamine, iminium, Lewis acid, Brønsted acid, Lewis base or Brønsted base catalysis and additives that improved its catalytic efficiency. The exact mode of action of 60 the additive depends on the particular catalytic system. For example, the additive may alter the electronic and steric properties as well as the chiral environment of an organocatalytic centre in a similar fashion as the ligand - ligand additive complexes presented in section 4. To determine the exact role of the additive as well as 65 the nature of the non-covalent interactions involved in the selectivity-determining step requires arduous mechanistic investigation. In addition, direct interactions between the additive and the substrate which are plausible in many cases, complicate the analysis.

70 Iminium catalysis is particularly influenced by the presence of anions than can interact with the cationic catalyst ensemble and the positively-charged transition state. ${ }^{468}$ Usually, the ion pair acting as catalyst is obtained by in situ mixing of an amino catalyst and an acid. List demonstrated that ion pairs composed by a achiral 75 iminium cation/ and a chiral anion ${ }^{533,534}$ (the so-called asymmetric counteranion directed catalysis, ACDC) or a chiral iminium cation and a chiral anion ${ }^{535}$ are efficient catalysts 


\section{Cite this: DOI: 10.1039/c0xx00000x}

\section{www.rsc.org/xxxxxx}

ARTICLE TYPE

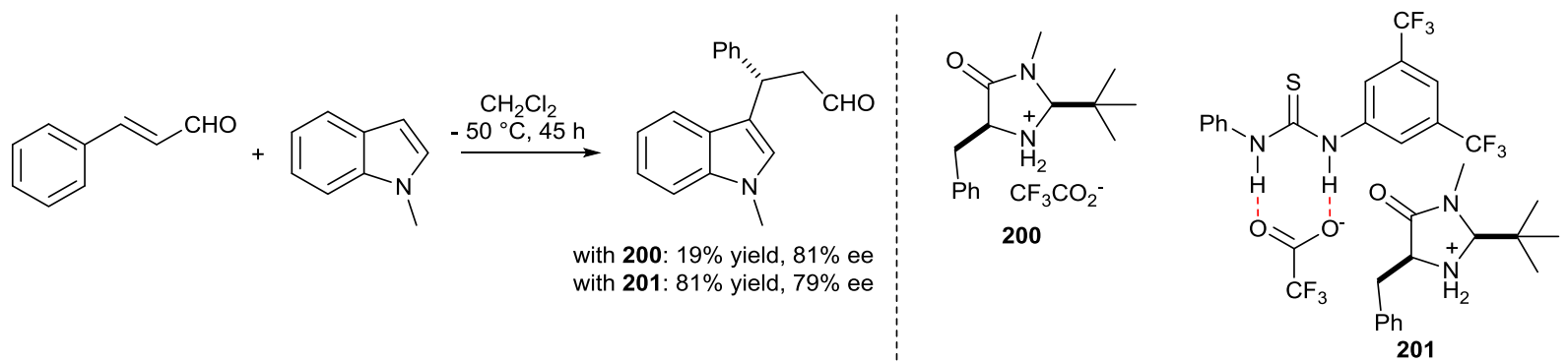

Fig. 52 Hydrogen bond donors enhance the reactivity of iminium-based catalysts. The reactivity of imidazolidinone catalysts is enhanced by the presence of a thiourea additive. The iminium ion derived from $\mathbf{2 0 1}$ is four times more active than the iminium derived from $\mathbf{2 0 0}$, because the latter is in close contact with the trifluoroacetate anion. Ref: see the text.

5 for a variety of reactions. ${ }^{52}$ The formation of a close-contact ion pair between the iminium ion and the additive is required to get high selectivity. This approach mimics the transfer of chirality between a chiral anion and an achiral cationic metal catalyst exemplified by Toste and others (see section 6.1). In a totally 10 different optic, $\mathrm{Xu}$ and co-workers recently demonstrated that the presence of thiourea additives in iminium-catalysed reactions facilitates the formation of the dissociated iminium active species by selective bonding with the anionic counterpart (a carboxylate anion, Fig. 52). ${ }^{536}$ As a result of the enhanced dissociation of the 15 iminium cation of the ion pair, better yields were obtained without significant loss of selectivity. For example, in the asymmetric Friedel-Crafts alkylation reaction of $N$-methylindole with cinnamyl aldehyde catalysed by imidazolidinonium triflate $\mathbf{2 0 0}$, the presence of $N$-phenyl- $N$ '-[3,5-bis(trifluoromethyl)]phenyl 20 thiourea results in a 4 fold increase of the reaction rate while maintaining the same enantioselectivity (compare catalysts 200 and 201, Fig. 52).
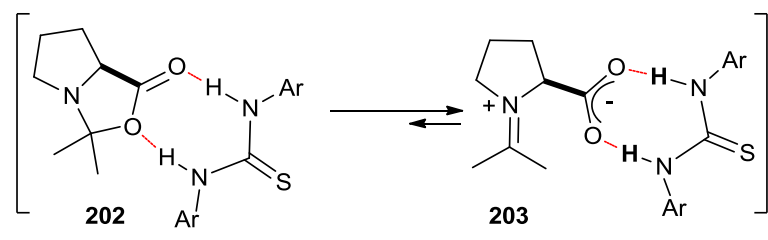

Fig. 53 Possible role of the thiourea additive for proline-catalysed

25 reactions. Binding of the oxazolidinone intermediate displaces the equilibrium towards the formation of the iminium ion. Ref: see the text.

Enamine catalysis also benefits from the presence of acid additives, but the effect is probably related to the direct activation of the electrophile by the acid. ${ }^{537,538} \mathrm{~A}$ great deal of work has been 30 directed to overcome the current limitations of the use of proline as catalyst (high catalyst loading required due to side reactions, poor solubility) while keeping intact its overall functional architecture. In 2006, Zhou et al. showed that the enantioselectivity delivered by proline catalyst for aldol reactions can be improved 35 by adding simple chiral diols (such as $(S)$-BINOL). ${ }^{539}$ In the proposed transition state, the chiral diol is hydrogen-bonded to the $\mathrm{COOH}$ group of the enamine intermediate and to carbonyl oxygen of the incoming electrophile. Demir and co-worker found that the addition of the Schreiner thiourea $(N, N$ '-bis[3,540 bis(trifluoromethyl)]phenyl thiourea) to proline increased the activity and selectivity of aldol reactions ${ }^{540}$ and others reactions ${ }^{541}$ performed in non-polar solvents. Based on the spectroscopic observation of a 1:1 complex formed between the carboxylate anion of proline and the $\mathrm{N}-\mathrm{H}$ protons of the thiourea, the authors 45 proposed that the strong bonding of the thiourea: i) aids the solubilisation of the proline catalyst, and ii) stabilises the intermediates or the transition state. Rios and co-workers also found a beneficial effect of thiourea additives for the prolinecatalysed desymmetrization of prochiral cyclohexanones. ${ }^{542}$ They 50 proposed a transition state in which the hydrogen bonds established between the $\mathrm{N}-\mathrm{H}$ protons of the thiourea and the oxygen atoms of the carboxylic group of proline: i) increase the acidity of the carboxylic group and, ii) stabilise the "chair" conformation of the List-Barbas-Hook six-membered ring transition state (Fig. 50). ${ }_{55}$ Upon reinvestigation of the mechanistic pathway reported by Demir, El-Hamdoumi et al. unraveled a more complex role of the thiourea additive. ${ }^{543}$ The thiourea co-catalyst does not help the solubilisation of proline itself, it increases the concentration in solution of the iminium ion $\mathbf{2 0 3}$ (Fig. 53), a precursor of the active 60 enamine species, by stabilising an open structure of the oxazolidinone intermediate 202. A similar role has been proposed for bifunctional ureas used to increase the rate of proline-catalysed $\alpha$-aminoxylations. ${ }^{544}$ Additional contribution of the thiourea in the energy stabilisation of the transition state, as hypothesized ${ }_{65}$ previously by Demir ${ }^{540}$ and Rios, ${ }^{542}$ cannot be ruled out. Molecules displaying hydrogen bond donors are not the only potential additives for more efficient proline organocatalysis. For example, Bella et al. demonstrated that the addition of Lewis bases improved the yields and selectivities of proline-catalysed Michael additions 70 of aldehydes to 2-cyclohexen-1-one. The formation of an ion pair composed by the carboxylate group of the deprotonated enamine intermediate and the ammonium function of the protonated Lewis base is probably at the origin of the observed enhanced rates. ${ }^{545}$ Although supplementary mechanistic studies are clearly needed to 75 better understand the exact role of these additives, the 


\section{Cite this: DOI: $10.1039 / \mathrm{c0xx} 00000 \mathrm{x}$}
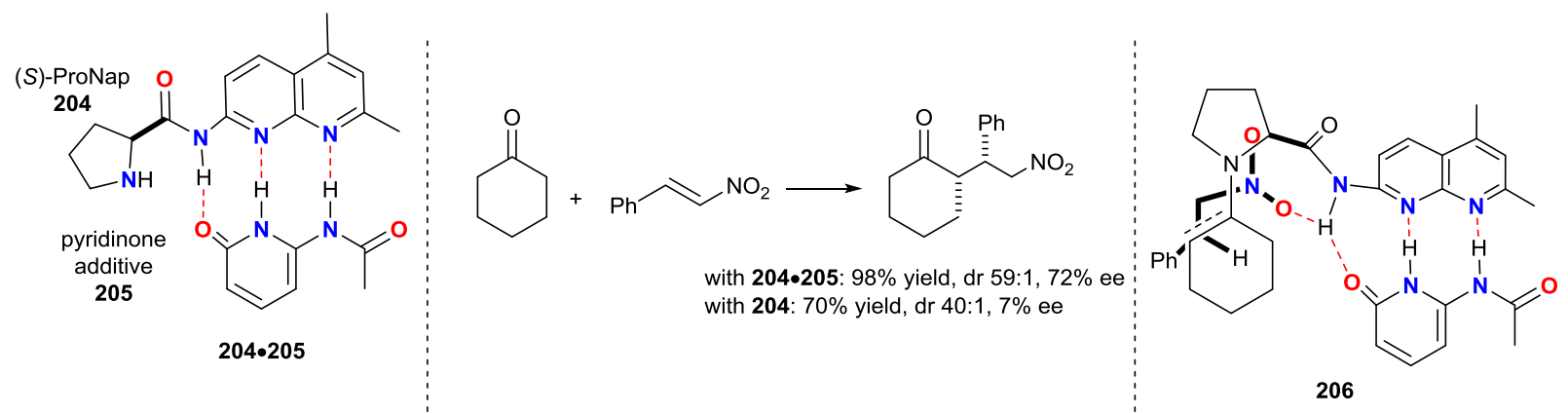

Fig. 54 The efficiency of enamine-based catalysts is enhanced by hydrogen-bonded additives. A supramolecular proline catalyst $\mathbf{2 0 4 \bullet 2 0 5}$ formed through the self-assembly of complementary fragments 204 and 205. Representation of a probable transition state 206 with a bifurcated hydrogen bond between the proline side-arm, the co-catalyst and the nitro substrate. Refs: see the text.

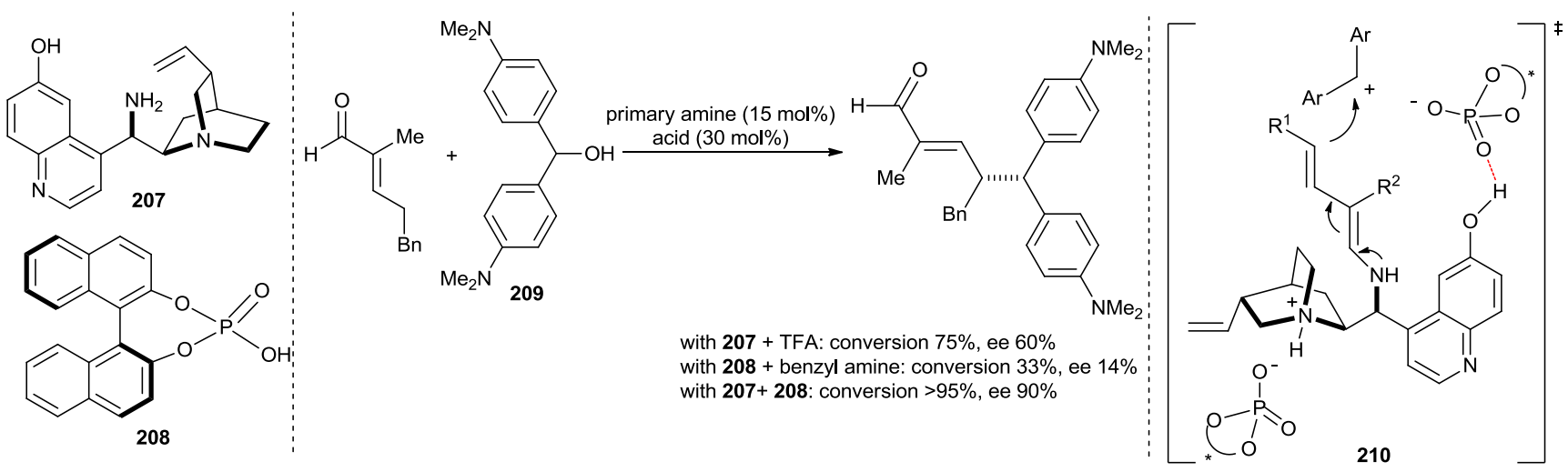

Fig. 55 The efficiency of dienamine-based catalysis is enhanced by the presence chiral phosphoric acid additives. Synergistic effect for the dienamine activation of $\alpha$-branched enals with a combination of a cinchona alkaloid derivative $\mathbf{2 0 7}$ and a chiral phosphoric acid 208. Right: proposed transition state 210. Ref: see the text.

strategies involving proline - additive combinations and the 10 extension to other amino acids, ${ }^{546}$ pave the way to promising classes of MDOs.

The modification of the catalytic properties of structurally more complex proline derivatives has also been investigated by means of the same strategy. Clarke et al. demonstrated that the chiral 15 environment delivered by a proline derivative, functioning as organocatalyst, can be modified through the formation of a hydrogen-bonded assembly with achiral additives. The work represents a nice example of a self-assembled modular organocatalyst. (S)-proline derivative 204 covalently 20 functionalized with an amidonaphthyridine group (Fig. 54) is an unselective catalyst for the Michael reaction between cyclohexanone and $\beta$-nitrostyrene. ${ }^{547}$ However, a remarkable improvement of enantioselectivity (from 7 to $72 \%$ ee) was observed in the presence of additives i.e. 205 containing a 25 pyridinone unit complementary to the hydrogen bonding motif of the amidonaphthyridine binding site equipping catalyst 204. The catalytic turnover and the diastereoselectivity of the reactions were also increased in the presence of the additives. The initial working hypothesis was that $\mathbf{2 0 4}$ and the additive $\mathbf{2 0 5}$ self-assembled into a 30 single supramolecular catalyst $\mathbf{2 0 4} \mathbf{2 0 5}$. The supramolecular catalyst 204-205 inhibits the reversible Michael addition and provides a better defined chiral environment than 204 alone. Further investigations with similar self-assembled modular systems but based on more soluble ureoimidazoles co-catalysts 35 suggest that: i) the co-catalyst displaces the equilibrium towards the formation of the enamine by assisting elimination of water from the intermediate $N, O$ hemiacetal and, ii) in the transition state (206, Fig. 54), a bifurcated hydrogen bond is established between the $\mathrm{N}-\mathrm{H}$ proton of the ancillary amido group of the proline 40 derivative, one oxygen atom of the co-catalyst and one oxygen atom of the nitro group of the Michael acceptor; this interaction may explain the enhanced levels of diastereo- and enantioselectivity exhibited by the self-assembled catalyst. ${ }^{548}$

In dienamine catalysis, ${ }^{549}$ a secondary amine catalyst activates ${ }_{45}$ an $\alpha, \beta$-unsaturated ketone affording $\gamma$-functionalization with various electrophiles. Melchiorre and co-workers have extended the scope of the reaction to $\alpha$-substituted linear $\alpha, \beta$-unsaturated aldehydes. ${ }^{550}$ Upon activation with the cinchona alkaloid derivative 207 (Fig. 55), $\alpha$-branched enals are alkylated in their $\gamma$ ${ }_{50}$ position in presence of bis(4-dimethylaminophenyl)methanol 209. A Brønsted acid is required to generate the stabilised carbocation derived from 209 acting as alkylating electrophilic 


\section{Cite this: DOI: $10.1039 / \mathrm{c0xx00000x}$}

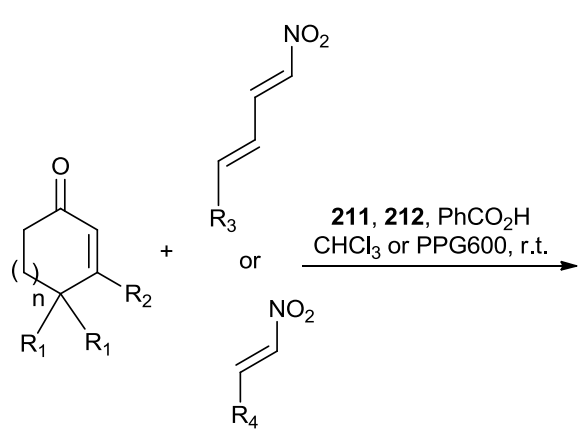

and poly(alkene glycol)s for organocatalytic Diels-Alder
species. In the presence of either $\mathbf{2 0 7}$ or the chiral phosphoric acid $\mathbf{2 0 8}$, the reaction is poorly selective. When combined together, a synergistic effect is observed and the best combination provides a series of $\gamma$-alkylated products with up to $95 \%$ ee. The cooperative effect is also highlighted by the observation of $10 \mathrm{matched} / \mathrm{mismatched}$ combination between the chiral cinchona alkaloid derivative and the chiral phosphate anion. Regarding the reaction mechanism, the authors proposed a dual role of the phosphate acting as the anion in both the ion pair with the protonated quinuclidine and in the ion pair with the benzhydryl 15 cation formed in situ. It is worthy to note that the hydroxyl group located at the 6'-position of the quinoline ring of $\mathbf{2 0 7}$ is required to achieve high conversion and selectivity. Accordingly, a hydrogen bond interaction between the phosphate anion and this hydroxyl group seems to be responsible for the formation of a highly20 organised transition state (210, Fig. $\mathbf{5 5})$.

In analogy with the examples of organometallic catalysts possessing a binding site, bifunctional organocatalysts which combine the reactive centre with a open-cavity suitable for substrate encapsulation will be mentioned in Part 2. The inclusion 25 of proline derivatives within the hydrophobic pocket of cyclodextrins proved to be a successful strategy to perform catalytic reactions in/on water and generate recyclable catalysts. ${ }^{551-553}$

$\mathrm{Xu}$ et al. prepared supramolecular catalysts by a self-assembly 30 process that combined a chiral pyrrolidine appended with a Lewis base (a pyridyl group) and poly(ethylene glycol)s (PEGs) or poly(propylene glycol)s (PPGs) in the presence of an acid. ${ }^{554,555}$ The supramolecular organocatalysts obtained were tested in DielsAlder reactions of cyclohexenones with nitrodienes affording 35 excellent levels of chemo-, regio- and enantioselectivities. ${ }^{554} \mathrm{UV}$ vis, NMR and CD analyses suggest that the acid has a dual role: i) it favours the self-assembly by maintaining the electrostatic interaction between the pyridinium cation and the polyether chain

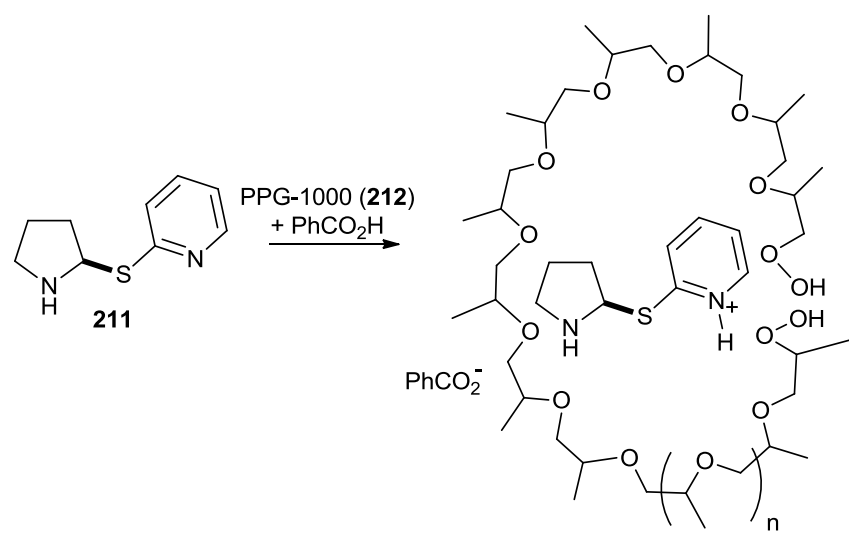

213 


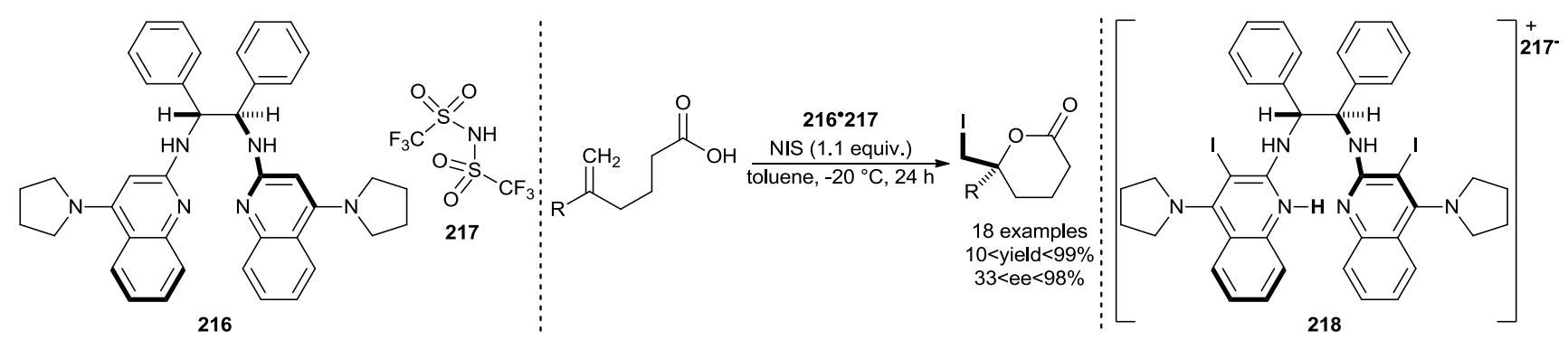

Fig. 58 The catalytic efficiency of a Brønsted acid catalyst (triflimide, 217) is enhanced by the presence of the bis(amidine) Brønsted base 216. The acidbase reaction between these two components generates a chiral proton catalyst that is effective for the enantioselective $\gamma$-lactonisation of various 5 unsaturated carboxylic acids. The active ion-paired catalyst $\mathbf{2 1 8}$ is thought to be the monoprotonated bisamidine with a triflimidate counteranion. NIS $=N$ iodosuccinimide. Refs: see the text.

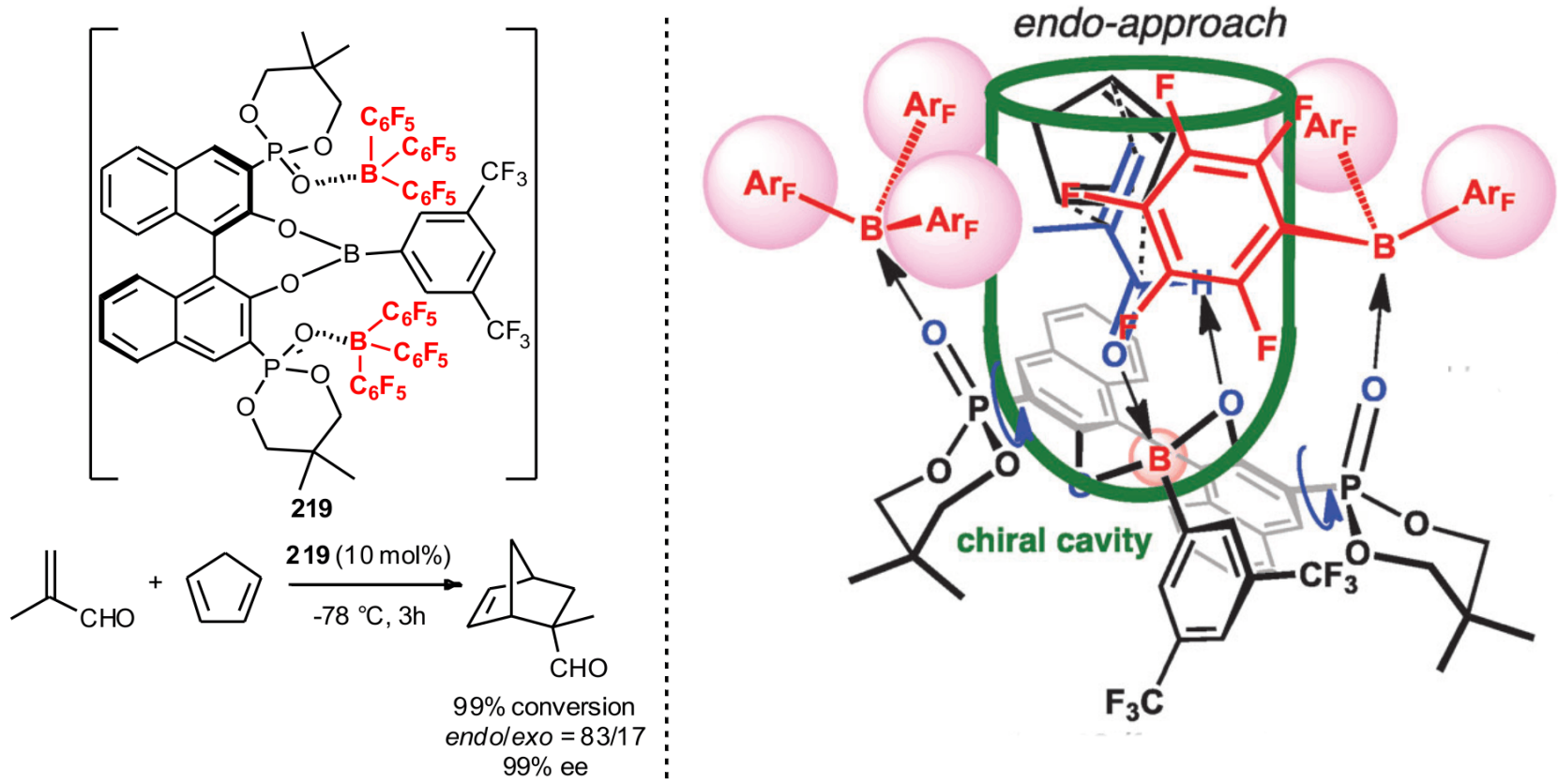

Fig. 59 Coordination bonds used to tune the activity and selectivity of Lewis acid catalysts. Left: Structure of the supramolecular boron-based catalyst 219. Catalyst 219 provides anomalous selectivity for the Diels-Alder reaction between cyclopentadiene and methacrolein: the enantiopure endo product is 10 formed in high yield. Right: A possible transition state for this reaction that rationalizes the formation of the endo-product. (The transition state is reprinted with permission from ref. 562. Copyright 2012. Royal Society of Chemistry). Refs: see the text.

substituents in its 3,3'-positions. Optimisation is also facilitated by the possibility of screening a high variety of achiral secondary amines. Tuning the acidity of Brønsted acid catalysts by adding a 15 weak Brønsted base is a well-known strategy in organic synthesis, however the design of acid catalysts that can deliver chiral proton is far more challenging. Johnston et al. developed a series of chiral proton catalysts that are readily made by mixing one equivalent of a chiral bisamidine ligand (such as 216, Fig. 58) and one equivalent 20 of an achiral strong acid. The prepared organocatalysts are bifunctional and consist in 1:1 ion pairs that are very effective for enantioselective aza-Henry reactions ${ }^{556,557}$ and lactonisation reactions. ${ }^{558}$ In the latter case, the combination of $\mathbf{2 1 6}$ and triflimide 217 achieves highly enantioselective (up to $98 \%$ ee) $\gamma$ 25 lactonisation reactions of unsaturated carboxylic acids in the presence of 1 equiv of $N$-iodosuccinimide (Fig. 58) ${ }^{558}$ The nature of the achiral anion of the ion pair was used to increase the level of enantioselectivity that the protonated chiral amine provides to the reaction (see the postulated active species $\mathbf{2 1 8}$, Fig. 58).
30 In the past, several examples have demonstrated the possibility to tune the acidity of a catalyst following the acid-assisted Lewis acid catalysis strategy developed by Kobayashi, Ishihara and others. ${ }^{559,560}$ The group of Ishihara used this strategy in order to finely tune the electronic and steric properties as well as the chiral 35 environment of a Lewis acidic boron-based catalyst. A series of three-component organocatalysts were prepared in situ. The catalysts were assembled by taking advantage of the formation of the boron BINOLates and included one BINOL derivative, one phenyl boronic acid and two borane units. ${ }^{561}$ Intermolecular dative 40 coordination bonds between the two $\mathrm{P}=\mathrm{O} \cdots \mathrm{B}\left(\mathrm{C}_{6} \mathrm{~F}_{5}\right)_{3}$ molecular components are critical in the design of the conformationally flexible complex (219, Fig. 59). The $\mathrm{B}\left(\mathrm{C}_{6} \mathrm{~F}_{5}\right)_{3}$ component played a dual role: i) due to its bulkiness it shaped a narrow and deep cavity around the Lewis acidic boron centre and, ii) it also 45 increased the Lewis acidity of the central boron atom through conjugated bonds. ${ }^{560}$ The energetically 


\section{Cite this: DOI: $10.1039 / \mathrm{c0xx00000x}$}

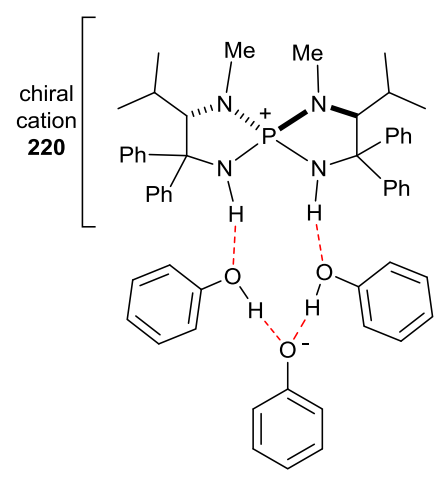

catalyst 221

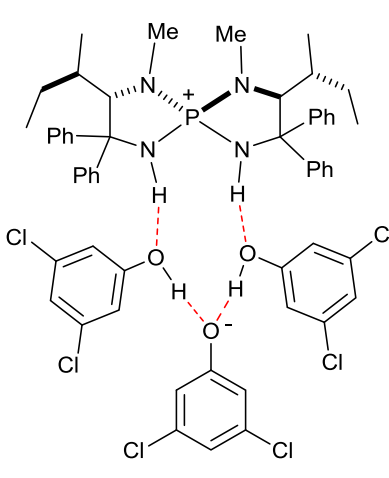

catalyst 222

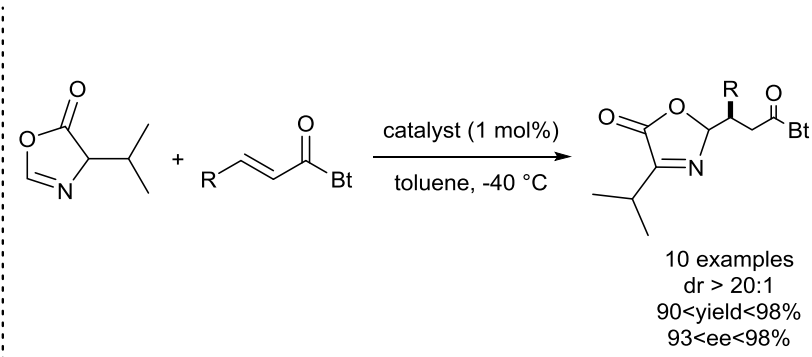

for $\mathrm{R}=\mathrm{Ph}, \quad$ with $221: 99 \%$ yield, $60 \%$ ee with 222: $95 \%$ yield, $95 \%$ ee

Fig. 60 Chiral supramolecular Brønsted base catalysts assembled through a hydrogen-bonding network. Bt = 1-benzotriazole. Ref: see the text.

demanding rotations around the $\mathrm{C}-\mathrm{P}$ and $\mathrm{P}=\mathrm{O} \cdots \mathrm{B}\left(\mathrm{C}_{6} \mathrm{~F}_{5}\right)_{3}$ bonds provided the supramolecular catalyst with a reduced 5 conformational flexibility. The Diels-Alder reaction between cyclopentadiene and methacrolein was conducted in the presence of $219(10 \mathrm{~mol} \%)$ in dichloromethane at $-78^{\circ} \mathrm{C}$ for $3 \mathrm{~h}$. The "anomalous" endo adduct was obtained as the major product of the reaction in high yield $(99 \%$ yield, endo/exo $=83 / 17)$ and with 10 excellent enantioselectivity ( $99 \%$ ee). Repulsive steric interactions between the fluorobenzene rings of $\mathbf{2 1 9}$ and the cyclopentadiene disfavoured the exo approach to occur inside the cavity (Fig. 59 shows a schematic representation of the transition state favouring the endo-selectivity). Conversely, the Diels-Alder reaction 15 between cyclopentadiene and acrolein in the presence of $\mathbf{2 1 9}$ afforded the "normal" endo product with high selectivity. Interestingly, a change in the BINOLate module in 219 yielded a new catalyst which gave predominantly the "anomalous" exo product in the reaction of cyclopentadiene and acrolein. The 20 generation of a shallower and wider cavity was put forward to explain the reaction outcomes. Taken together, these results pointed out the ability of well-chosen non-covalent interactions to tune the size and shape of an internal cavity having a direct impact on its catalytic performance. ${ }^{52}$ This strategy presents some 25 analogy with the encapsulation of rhodium catalytic centres by means of non-covalent interactions (see 44, Fig. 11 and 81•713, Fig. 23), which exhibit unusual selectivities in hydroformylation reactions.

Ooi and co-workers reported an unusual example that enables 30 the fine tuning of the catalytic performance of a self-assembled catalyst containing a Brønsted base centre. ${ }^{563}$ The supramolecular organocatalyst 221 was prepared through the self-assembly process of four molecular components: a chiral phosphonium cation (220), two molecules of phenol and one phenolate anion.

35 The four-particle assembly is stabilised through hydrogen bonds, ionic hydrogen bonds and charge-charge interactions (221, Fig. 60). The catalyst was tested in the asymmetric Michael reaction between a particular azlactone and various $\alpha, \beta$-unsaturated ketones. The azlactone was deprotonated by the organocatalyst 40221 functioning as a supramolecular base and the corresponding enolate was incorporated in the hydrogen-bonded network as a substitute of the phenoxide component without significant disruption of the aggregate's geometry. The best results derived from the use of a phenyl derivative as the Michael acceptor 45 providing a diastereomeric ratio higher than 20:1 for the two possible $1: 4$ adducts and a $60 \%$ ee for the major diastereoisomer. It was also demonstrated that the selectivity of the reaction could be tuned through structural modifications of the achiral phenolic components. Particularly, the use of 3,5-dichlorophenol induced a 50 significant increase in enantioselectivity. In addition, structural modifications of the chiral cationic moiety were also crucial to achieve the highest selectivity. These observations are in support of the idea that all of the components of the catalytic assembly play an important role in the transfer of chiral information to the 55 transition states. The supramolecular catalyst 222 afforded a $95 \%$ yield and 95\% ee (Fig. 60). It is remarkable that the order of addition of the various components do not affect the catalytic performance of the self-assembled catalyst. This methodology enables a rapid screening of Brønsted base catalysts through the 60 sequential modification of one of the building blocks of the selfassembly at a time.

The examples mentioned in this section clearly demonstrate that non-covalent interactions can be successfully employed for the construction of dynamic bifunctional catalysts as well as to tune ${ }_{65}$ the electronic, steric properties and the chiral environment of organocatalysts whatever their mode of actions (amino-based, Lewis acid, Brønsted acid and Brønsted base). The self-assembly nature of MDOs enables the easy preparation of cooperative and highly modular catalysts. The outstanding properties of MDOs 70 augur well for the fast development of this area in the burgeoning field of organocatalysis. 


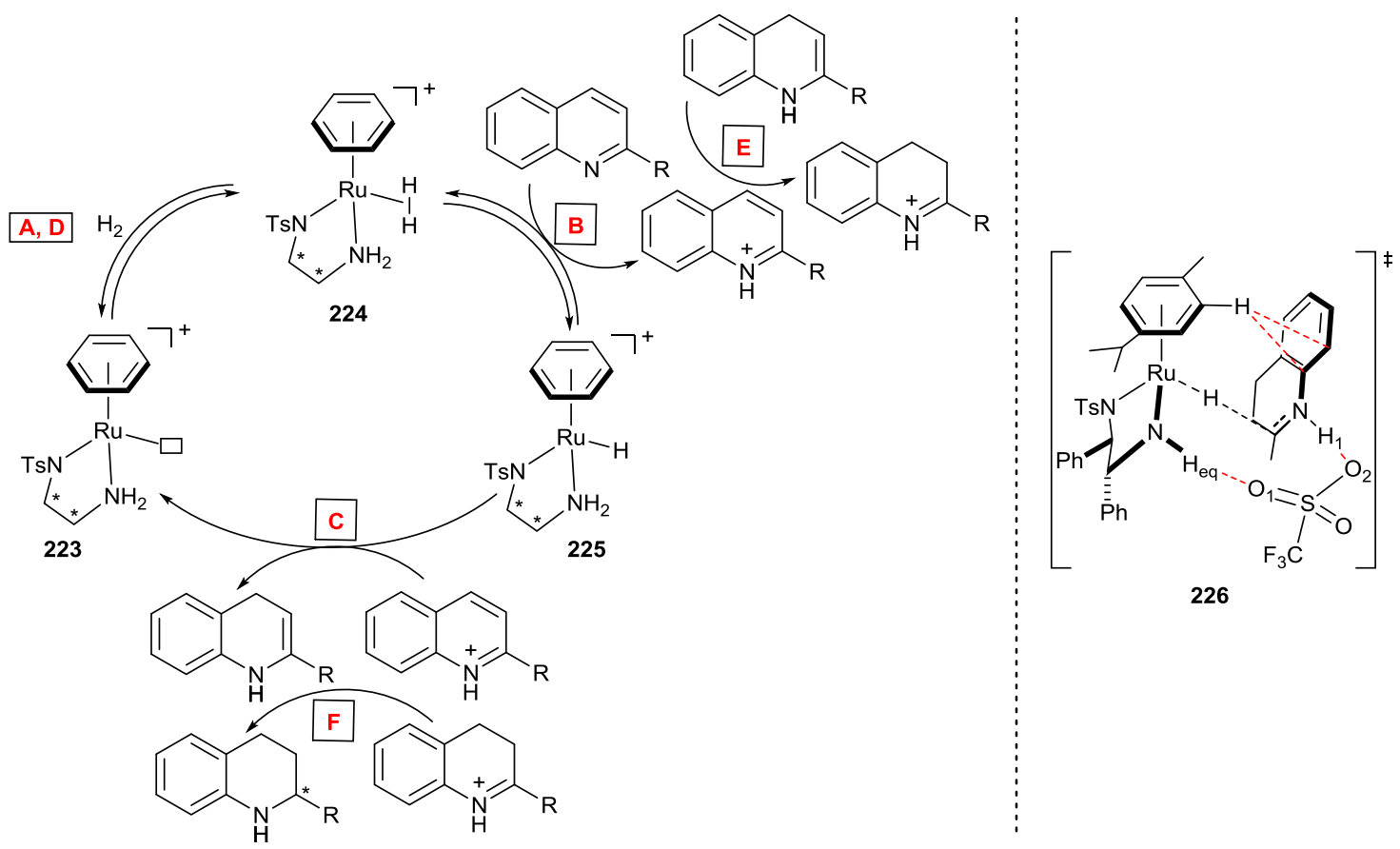

Fig. 61 Example of metal - achiral anion - substrate interactions that govern the selectivity of a reaction. Left: proposed mechanism for the hydrogenation of quinolines with $\left\{\mathrm{Ru}(\mathrm{OTf})\left(\eta^{6}\right.\right.$-arene $)[(S, S)$-TsDPEN $\left.]\right\}$, the phenyl groups of the ethylenediamine ligands and OTf are omitted for clarity. Right: Proposed transition state for the enantioselectivity-determining step. Refs: see the text.

\section{Multiple interactions}

\subsection{Metal - anion - substrate interactions}

\subsubsection{Cationic metal with achiral counteranion}

From a general viewpoint, counteranions of cationic metal 10 catalysts compete with the substrate for the coordination of a free site and, as a result, the formation of a close contact ion-pair is detrimental to catalytic activity. The role of the counteranion in terms of selectivity is far less intuitive and a positive effect of loose metal - anion pairs has been demonstrated mostly in the field of 15 olefin polymerization. ${ }^{71}$

Two examples were reported recently for which the achiral counteranion does not simply act as a metal partner, but it is also involved in a selectivity-determining interaction with the substrate. Fan and co-workers found that $\left\{\mathrm{Ru}(\mathrm{OTf})\left(\eta^{6}\right.\right.$-arene $)[(S, S)$ 20 TsDPEN] $\}$ is a very efficient catalyst for the asymmetric hydrogenation of quinolines in ionic liquids ${ }^{564}$ or under solventfree conditions. ${ }^{565}$ In organic solvents, the substrate scope has been extended to 2-arylquinolines, and to 2-functionalized and 2,3disubstituted quinoline derivatives. ${ }^{352}$ The reaction is strongly 25 altered by the nature of the anion coordinated to the precatalyst: $\left[\mathrm{Ru}(\mathrm{OTf})\left(\eta^{6}\right.\right.$-arene)(TsDPEN)] is very efficient whereas the analogous chloride complex is almost completely inactive. First investigation of the mechanism reveals an ionic catalytic pathway different from the classical outer-sphere mechanism observed for
30 the hydrogenation and transfer-hydrogenation of ketones with this catalyst (see section 5.1.2.1). A series of stoichiometric experiments between potential $\mathrm{Ru}$ intermediates and quinoline or protonated quinoline substrate showed that the hydrogen addition undergo a stepwise $\mathrm{H}^{+} / \mathrm{H}^{-}$transfer process outside the coordination 35 sphere. ${ }^{352}$ Deuterium-labelling experiments and DFT calculations suggest a sequential ionic pathway for quinoline hydrogenation that involves 1,4-hydride addition, isomerisation and 1,2-hydride transfer. A representation of these interconnected catalytic cycles is given in Fig. 61; the main steps are: i) the reversible formation 40 of a dihydrogen complex $\mathbf{2 2 4}$ from $\mathbf{2 2 3}$ (steps A and D), ii) the deprotonation of the dihydrogen ligand by the quinoline that generated both the active species $\mathbf{2 2 5}$ and the activated substrate (the protonated quinoline; step B), iii) a 1,4-hydride transfer that affords the enamine and regenerates 223 (step C), iv) the 45 deprotonation of the dihydride ligand by the enamine (isomerisation step E) that yields the iminium and the ruthenium hydride intermediate $\mathbf{2 2 5}$ and, v) the irreversible 1,2- hydride transfer between $\mathbf{2 2 5}$ and the activated iminium give the products and 223 (step F) that can further react with $\mathrm{H}_{2}$. DFT calculations ${ }_{50}$ performed on the enantioselectivity-determining step suggest a well-organised transition state involving the Ru hydride complex 225, the activated iminium substrate and the triflate anion. The triflate anion form two hydrogen bonds: one with the $\mathrm{N}-\mathrm{H}$ proton of the amino ligand $\left(\mathrm{H}_{\mathrm{eq}} \bullet \bullet \mathrm{O}_{1}\right)$ and the other with the $\mathrm{N}-\mathrm{H}$ proton 55 of the iminium substrate $\left(\mathrm{H}_{1} \bullet \bullet \mathrm{O}_{2}\right)$, the overall 

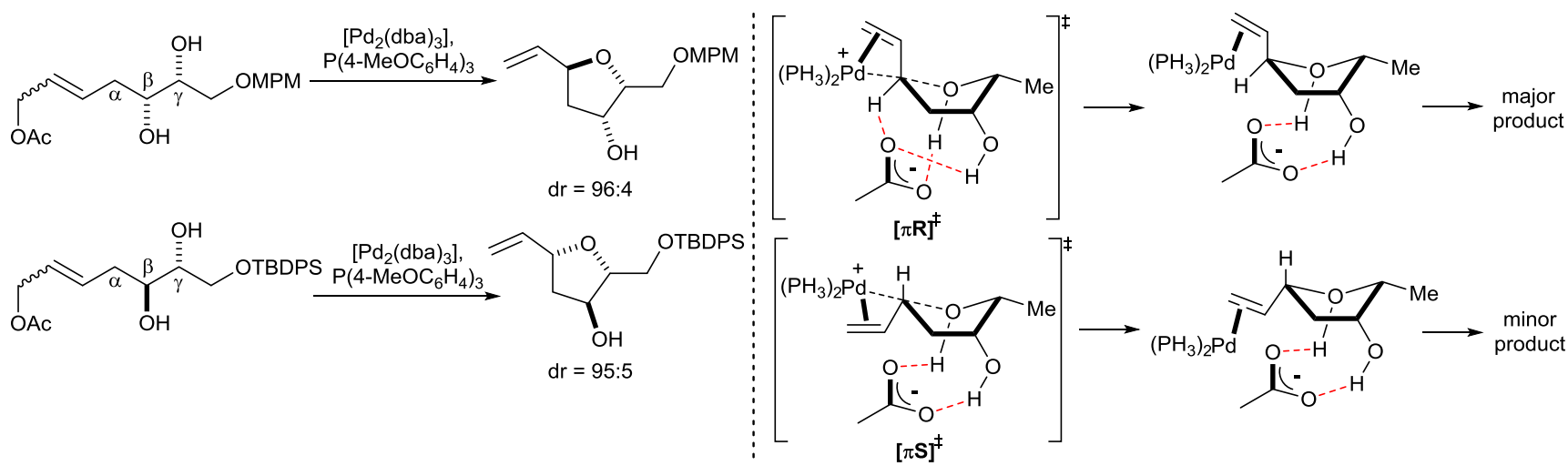

Fig. 62 Hydrogen bon interactions within a metal - achiral anion - substrate ternary complex mediate the selectivity of intramolecular reactions. Left: high diastereomeric ratios obtained during the palladium-catalysed intramolecular cyclization of $\beta, \gamma$-dihydroxyl allyl acetates. Right: proposed transition 5 states for these intramolecular Tsuji-Trost reactions: compared to $[\pi \mathrm{S}]^{\ddagger},[\pi \mathrm{R}]^{\ddagger}$ is further stabilised by a strong hydrogen bond between the allylic proton and the acetate anion. MPM = methoxyphenylmethylidene, TBDPS $=$ tert-butyldiphenylsilyl. Ref: see the text.
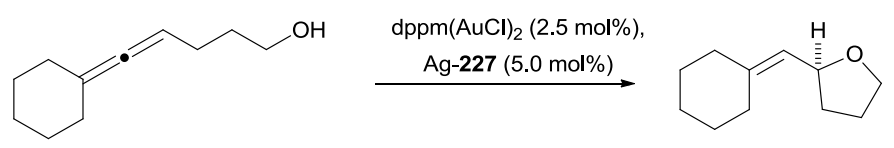

$90 \%$ yield, $97 \%$ ee

(R)-BINAP(AuCl $)_{2}, \mathrm{AgBF}_{4}: 52 \%$ yield, $6 \%$ ee<smiles>CC(C)=C=CCCC(=O)O</smiles>

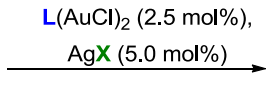

$\mathrm{L}=(R)-\mathrm{BINAP} ; \mathrm{X}=4-\left(\mathrm{NO}_{2}\right)-\mathrm{C}_{6} \mathrm{H}_{3}-\mathrm{COO}^{-}$

$\mathrm{L}=\mathrm{dppm} ; \mathrm{X}=\mathbf{2 2 7}$

$\mathrm{L}=(R)$-BINAP; $\mathrm{X}=\mathbf{2 2 7}$

$\mathrm{L}=(S)$-BINAP; $\mathbf{X}=\mathbf{2 2 7}$

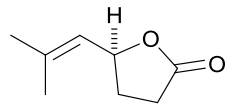

$80 \%$ yield, $38 \%$ ee $(R)$

$89 \%$ yield, $12 \%$ ee $(S)$

$91 \%$ yield, $3 \%$ ee $(R)$

$88 \%$ yield, $82 \%$ ee $(S)$
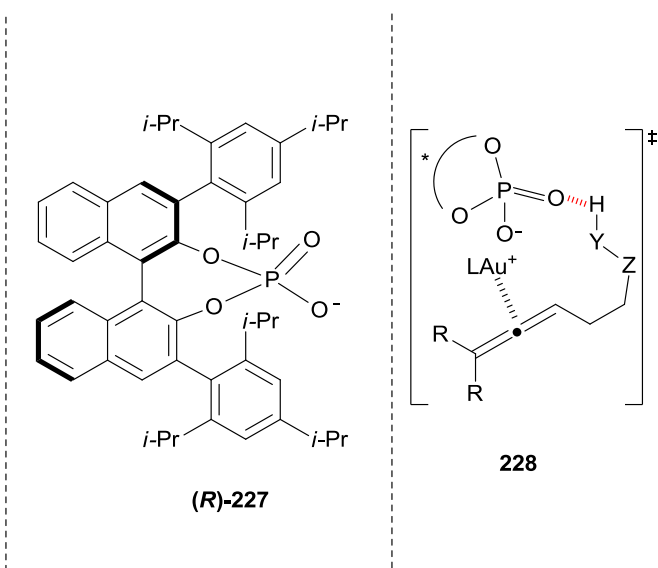

Fig. 63 Chirality induced by counteranion $(\boldsymbol{R})-\mathbf{2 2 7}$ for $\mathrm{Au}^{\mathrm{I}}$-catalysed asymmetric reactions. The conjugate acid of $\mathbf{2 2 7}$ is named Trip (for 3,3'-bis(2,4,6triisopropyl- phenyl)-1,1'-BINAPhthyl-2,2'-diyl hydrogen phosphate) and Ag-227 is thus referred to as Ag-Trip. Right: a plausible three-component transition state that involves a hydrogen bond interaction between the phosphate anion and the substrate. Ref: see the text.

assembly forming a 10-membered ring (226, Fig. 61). The enantioselectivity originated from $\mathrm{C}-\mathrm{H}-\pi$ interactions between the $\eta^{6}$-arene coordinated to the Ru metal and the iminium substrate similarly to what was described by Noyori for ketone reduction 15 (see Fig. 32).

Gandon, Roulland and co-workers disclosed an unanticipated role played by the counteranion during the course of an intramolecular Tsuji-Trost reaction. ${ }^{56} \mathrm{~A}$ high degree of diastereoselectivity was observed during the cyclization of $\beta, \gamma$ 20 dihydroxyl allyl acetates $(\mathrm{dr} \geq 95: 5)$. The presence of both a hydroxyl group at the $\beta$-position of the allyl moieties and a counteranion with hydrogen bonding ability was found to be primordial to achieve a high degree of selectivity. Upon investigation of the transition states leading to the two
25 diastereoisomers by DFT, the acetate anion has been found to be involved in hydrogen bond interactions with both the $\mathrm{O}-\mathrm{H}$ protons of the substrate. However, an additional hydrogen bond established between the triflate anion and the allylic $\mathrm{C}-\mathrm{H}$ proton is observed for the $[\pi \mathrm{R}]^{\ddagger}$ transition state (compared $[\pi \mathrm{R}]^{\ddagger}$ and $[\pi \mathrm{S}]^{\star}$ transition 30 states represented in Fig. 62). This interaction stabilises the $[\pi R]^{*}$ transition state and furnishes the diastereoisomer that is observed experimentally.

These two examples reveal the non-innocent role that an achiral counteranion can play in mediating the selectivity of a metal35 catalysed reaction through the formation of a well-organised transition state. 


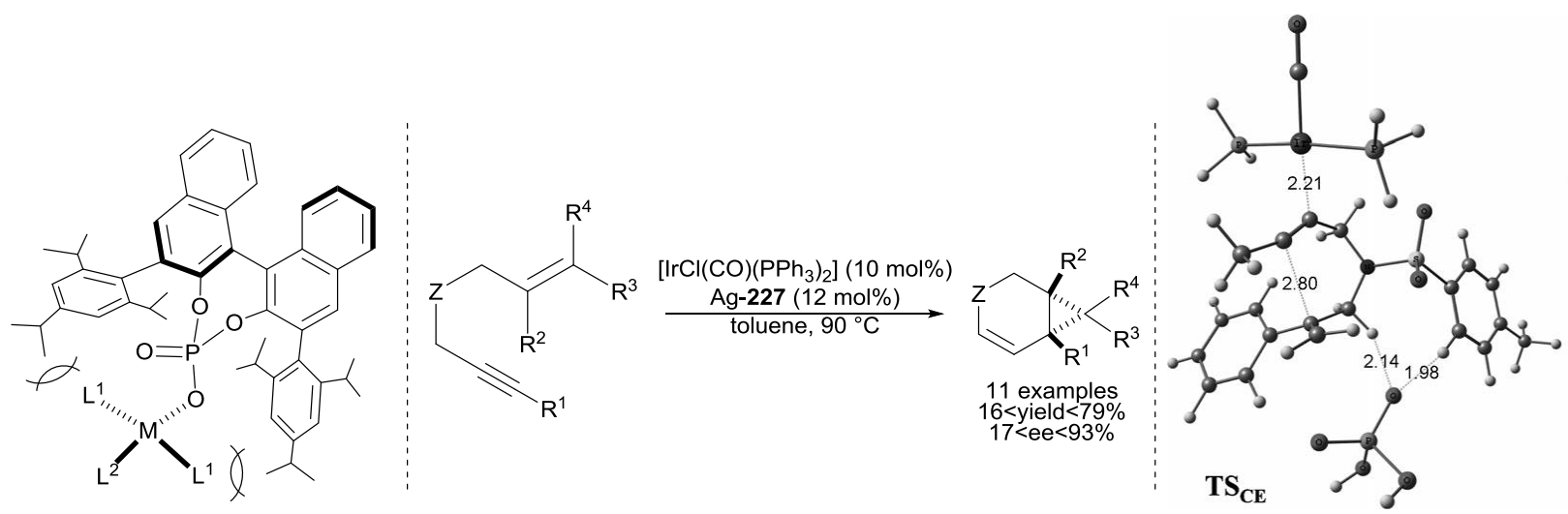

Fig. 64 Metal counteranion - substrate interactions in gold-catalysed reactions. Square planar complex with co-ligands $\mathrm{L}^{1}$ that disfavours the coordination of the phosphate anion due to steric repulsion. The combination of Vaska's complex $\left[\mathrm{IrCl}(\mathrm{CO})\left(\mathrm{PPh}_{3}\right)_{2}\right]$ and the silver complex of Trip $(\mathbf{A g}-\mathbf{2 2 7})$ is active 5 and selective for the carbocyclization of 1,6-enynes. Right: proposed structure for the 6-endo transition state (for computations, 227 has been replaced by the model phosphate $\mathrm{HPO}_{4}^{-}$). (The transition state is reprinted with permission from ref. 575. Copyright 2011 John Wiley and Sons).

\subsubsection{Cationic metal with chiral counteranion}

In classical metal-catalysed asymmetric reactions, a chiral ligand is coordinated to the metal and the stereoselection process 10 occurs in the first coordination sphere of the complex. Alternative approaches were investigated in order to avoid the use of expensive chiral ligands and to induce selectivity from remote stereogenic centres. One solution was provided by performing the reaction in a chiral ionic liquid phase. ${ }^{567,568}$ Alternatively, chiral anions can be 15 used to induce chirality to a cationic metal catalyst via electrostatic interactions. ${ }^{569-572}$

In recent years chiral ions have been used for the resolution of enantiomeric transition-metal complexes. ${ }^{573}$ In 2007, Toste et al. reported the use of chiral counteranions for several cationic gold20 catalysed reactions (Fig. 63). ${ }^{574}$ For hydroalkoxylation and hydroamination reactions, the achiral precatalyst with chiral anion 227 outperforms a series of gold complexes derived from a chiral diphosphine such as BINAP. For a more challenging substrate, a "matched" combination of $(S)$-BINAP and 227 as gold ligand and 25 counteranion, respectively, gave higher conversion and ee than the use of the chiral anion or $(S)$-BINAP alone (Fig. 63). The presence of $\mathrm{O}-\mathrm{H} / \mathrm{N}-\mathrm{H} \cdots \cdot \mathrm{O}$ hydrogen bonds between the phosphate counteranion and the substrates (see 228, Fig. 63) was not hypothesized in the initial paper but is very probable considering: 30 i) the large scope of nucleophiles that are tolerated by the gold chiral anion catalyst, and ii) that the same catalytic system is inactive for the carbocyclization of 1,6-enynes. Regarding the prospective coordination of the chiral anion to the gold atom, the strong substrate - anion interaction can facilitate the dissociation 35 of the $\mathrm{P}-\mathrm{O} \cdots A \mathrm{Au}$ bond, liberating a coordinating site for the substrate. Based on this last observation, Barbazanges et al. postulated that the use of a square planar complex will disfavour the coordination of chiral phosphate due to the steric repulsion of the co-ligands neighboring the potentially coordinated anion. ${ }^{575}$
40 Accordingly, they found that a combination of Vaska's complex $\left[\mathrm{IrCl}(\mathrm{CO})\left(\mathrm{PPh}_{3}\right)_{2}\right]$ and the silver complex of the Trip anion $(\mathbf{A g}-$ 227) is efficient for the carbocyclization of 1,6-enynes (up to $93 \%$ ee, Fig. 64). DFT calculations suggest that the 6-endo-dig cyclization pathway is more exergonic than the exo pathway. ${ }_{45}$ Attempts to bring the phosphate anion close to the cationic iridium centre resulted in systematic repulsion from the coordination sphere. Accordingly, the authors proposed a transition state for the 6-endo-dig cyclization pathway for which the phosphate anion is located in the second coordination sphere of iridium and interacts 50 with the substrate through two $\mathrm{C}-\mathrm{H} \bullet \bullet \mathrm{O}$ hydrogen bonds.

The Toste group applied the counteranion-directed catalysis strategy for the gold-catalysed preparation of enantioenriched heterocyclic systems (i.e. pyrazolidines, isoxazolidines, and tetrahydrooxazines) from allenes (ee up to $99 \%$ ). ${ }^{576}$

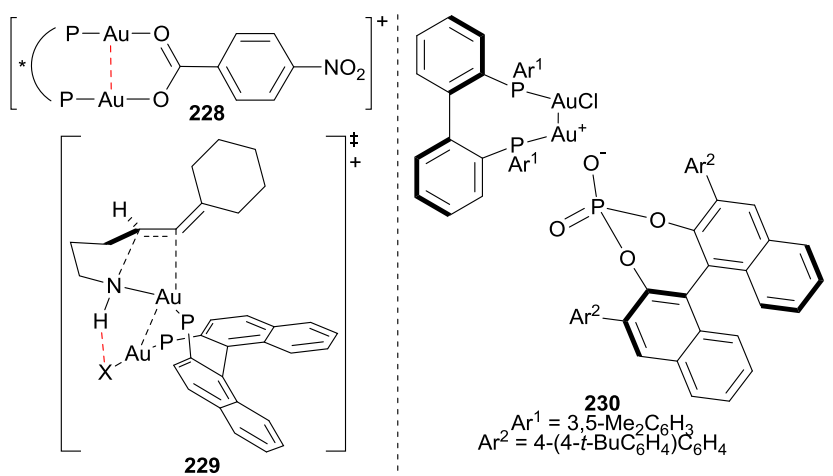

Fig. 65 Non-covalent chiral anion - substrate or chiral anion - metal interactions mediate the selectivity of Au-catalysed reactions. Left: The coordinated benzoate anion directs the substrate, favouring the synaddition pathway. Transition state $\mathbf{2 2 9}$ that leads to the experimentally60 observed enantiomer. Right: Ion-pairing catalyst used in the asymmetric hydroalkoxylation of allenes. Refs: see the text 


\section{Cite this: DOI: $10.1039 / \mathrm{c0xx00000x}$}
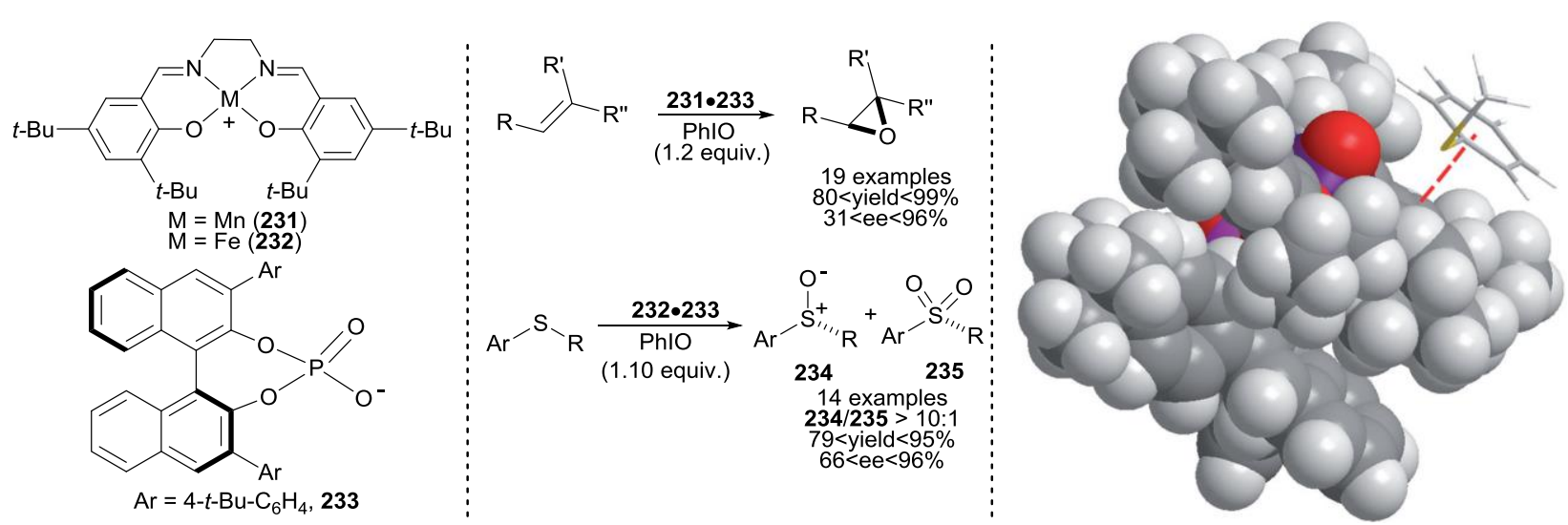

Fig. 66 Chiral anion-mediated asymmetric oxidation reactions. $231 \cdot 233$ and $\mathbf{2 3 2} \cdot \mathbf{2 3 3}$ are the best combinations for the asymmetric epoxidation of alkenes and the oxidation of sulfides respectively. Right: hypothetic 3D-model for the oxidation of the sulfide substrate by the iron-oxo ion-pairing catalyst. (The 5 model is reprinted with permission from ref. 581. Copyright 2012 John Wiley and Sons). Refs: see the text.

In 2007, the same group found that benzoate anions significantly increase the selectivity of the intramolecular hydroamination of allenes catalysed by dinuclear gold catalysts. ${ }^{577}$ The active species is a monocationic complex with a benzoate 10 anion bridging the gold atoms and the overall structure is further stabilised by an aurophilic interaction (see 228, Fig. 65). Kang, Lee and co-workers recently investigated the important role played by the coordinated benzoate anion. ${ }^{578}$ Based on DFT calculations, they found that a hydrogen bond established between the oxygen 15 atom of the benzoate anion and the $\mathrm{N}-\mathrm{H}$ proton of the substrate allows the nucleophilic attack to occur at the cis position to gold. This interaction is maintained along the catalytic pathway that leads to the $S$ enantiomer (the experimentally-observed one) whereas this interaction is weakened in the transition state that 20 leads to the less favoured $R$ enantiomer (see 229, Fig. 65). The study reveals the potential role of coordinating anions in mediating highly selective transformations. Previously, Mikami and coworkers observed a synergistic effect when a combination of $(R)$ BIPHEP-( $\mathrm{AuCl})_{2}$ and chiral phosphate anions are employed in the 25 hydroalkoxylation of allenes. ${ }^{579}$ Interestingly, the more active and selective species is again a monocationic gold complex (230, Fig. 65). The optimised catalyst provided the furan derivatives in high yields and enantioselectivities (up to $95 \%$ ee).

The chiral counteranion-directed catalysis strategy is not limited 30 to gold reactions. List et al. presented a revisited version of the Jacobsen-Katsuki catalyst, using a combination of an achiral Mnsalen complex and a chiral phosphate anion (up to $96 \%$ ee for the

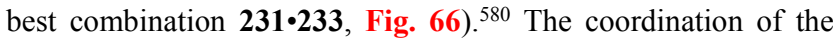
salen ligand to the cationic $\mathrm{Mn}$ centre generates two 35 enantiomorphic conformations; one of them is favoured upon the addition of a well-chosen chiral phosphate anion. The absolute configuration of the product is the same as the one observed with $S, S$ Jacobsen catalyst for which chirality is induced from the chiral salen backbone. The formation of an ion pair between the cationic $40 \mathrm{Mn}$ complex and the phosphate anion was further supported by the fact that polar solvents decrease the catalytic efficiency. Additional features of this original catalyst are that: i) no additional neutral ligand is required to increase the activity and, ii) the formation of a loose ion pair increases the electrophilicity of the metal centre, ${ }_{45}$ providing high activity. The same ion-pairing catalyst was also investigated for the asymmetric oxidation of methyl phenyl sulfide but it only provided modest enantio- and chemoselectivity (sulfoxide/sulfone ratio). ${ }^{581}$ The iron analogue $\mathbf{2 3 2} \cdot \mathbf{2 3 3}$ is less active and thus limits the amount of over-oxidation of the sulfoxide 50 into sulfone (Fig. 66). Non-covalent interactions between the incoming sulfide and the salen ligands generates a well-organised transition state (see the hypothetic 3D-model represented in Fig. 66). ${ }^{582}$ The alkyl chain of the sulfur points in an open space, explaining why the catalyst is particularly selective for sulfide 55 substrates that possess a long alkyl chain.

The initial breakthrough accomplished by Toste has clearly inspired a lot of research groups in the development of catalytic systems that combine an achiral metal cation and a chiral counteranion. In parallel, catalysts incorporating a metal complex 60 and a Brønsted acid (mainly chiral BINOL-derived phosphoric acids) have also been developed. With substrates containing basic sites, the acid can have a dual role: activating the substrate and acting as counteranion of the metal catalyst. A few examples of dual activation of substrates by a metal complex and a Brønsted 65 acid will be presented in the next subsection.

In the previous examples, the formation of an ion-pairing catalyst that is closely involved in the selectivity-determining step is made plausible by the corroboration of several experimental data: the dependence of the selectivity on the solvent polarity, the 70 observation of matched/mismatched combinations, etc... However, the presence of additional interactions between the 


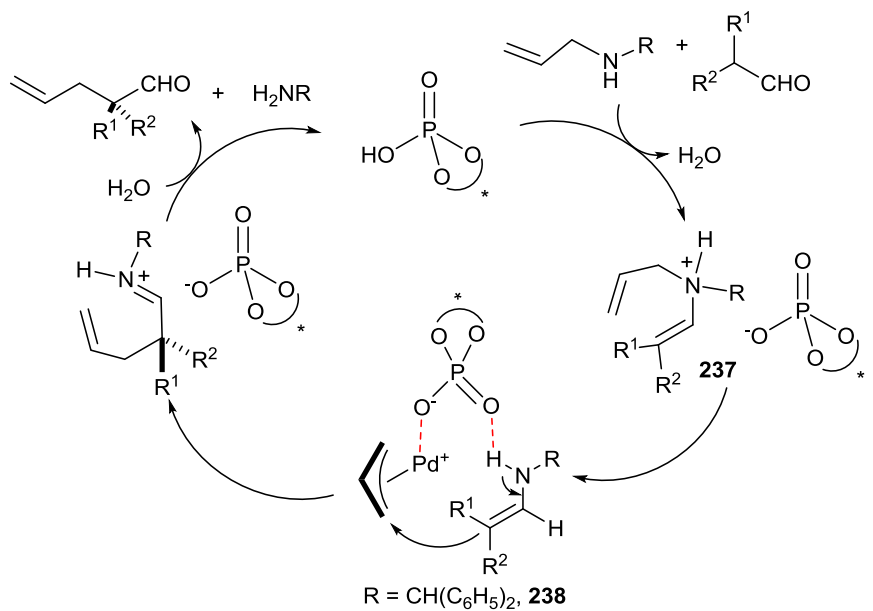

Fig. 67 Cooperative catalysis between an achiral metal catalyst and a chiral Brønsted acid catalyst. Left: $\alpha$-allylation of $\alpha$-branched aldehydes by a combination of a $\operatorname{Pd}(0)$ precursor and Trip. Right: reaction pathway for the $\alpha$-allylation of $\alpha$-branched aldehydes with 236. In the proposed hydrogenbonded transition state 238, the phosphate anion acts as a bridge between the enamine and the Pd-allyl intermediates. Refs: see the text.

5 substrate and the chiral anion and the possible coordination of the chiral anion to the metal cation at any step of the catalytic cycle cannot be discarded. Whatever the exact nature of the mechanism, ${ }^{583}$ the strategy has undoubtedly led to the discovery of innovative catalytic systems. ${ }^{584-591}$ Any metal-catalysed reaction 10 that involves a cationic intermediate in its stereo-determining step can benefit from the chiral counteranion-directed catalysis strategy.

\subsection{Metal catalyst - organocatalyst - substrate interactions}

The scope of the reactions and substrates that can be subjected to 15 catalytic activation has greatly increased by means of catalytic systems that combine a metal catalyst and an organocatalyst. ${ }^{471-475}$ The feasibility of the approach relies on the ability of the catalysts to co-exist and to activate the substrate(s) in the same reaction medium. A lot of systems have now been disclosed that differ by 20 the mode of activation of the substrate: i) cascade catalysis means that one substrate is transformed independently by one catalyst and the resulting product is transformed by the second catalyst (independent catalytic cycles), ii) dual catalysis refers to the simultaneous activation of one substrate by the two catalysts and, 25 iii) synergistic catalysis deals with the simultaneous activation of two substrates by two separate catalysts (intertwined catalytic cycles). Only the last two approaches allow for cooperative catalysis as a result of reciprocal attractive interactions between the two catalysts. A few examples have been disclosed for which non30 covalent interactions between the components of the reaction (the two catalysts and the substrate(s)) have been detected that may explain the observed cooperative effect.

List and co-workers developed an original system for the $\alpha$ allylation of $\alpha$-branched aldehydes, a particularly challenging class 35 of substrates in Tsuji-Trost allylation reactions. ${ }^{592}$ In their first approach, they used a combination of $\left[\mathrm{Pd}\left(\mathrm{PPh}_{3}\right)_{4}\right]$ and a chiral phosphoric acid ((R)-Trip), achieving up to $97 \%$ ee for the reaction (Fig. 67). The success of the reaction relies both on the use of a $N$ benzhydryl amine derivative (236, Fig. 67) and the dual role 40 played by the chiral phosphoric acid. The first step of the catalytic cycle is the acid-promoted reaction between the $N$-benzhydryl amine derivative 236 and the aldehyde substrate. The resulting enamonium phosphate salt 237 reacts with the $\operatorname{Pd}(0)$ precursor generating a cationic $\eta^{3}$-allyl-Pd-complex and an enamine. The 45 nucleophilic addition of the enamine onto the $\eta^{3}$-allyl-Pd complex yields an iminium phosphate salt. Upon hydrolysis, this salt releases the allylated product and $N$-benzhydryl amine (byproduct) as well as regenerates the phosphoric acid catalyst. Regarding the high degree of stereoinduction despite the use of an 50 achiral palladium precursor, a well-defined transition state $\mathbf{2 3 8}$ involving the enamine, the $\eta^{3}$-allyl-Pd complex and the phosphate anion has been proposed in which the phosphate anion acts as a counteranion of the palladium and a hydrogen bond acceptor for the enamine intermediate. The same catalytic combination was 55 tested for the direct $\alpha$-allylation of $\alpha$-branched aldehydes with allylic alcohols (Fig. 67). ${ }^{593}$ However, almost no selectivity was observed as a probable result of a mixture of $E / Z$-enol isomers generated under the reaction conditions. The authors hypothesized that the in situ formation of an enamine with a preferred $E$ 60 configuration will be beneficial. Indeed, after a screening of primary amines, they found that the combination of $(S)$-Trip, $\left[\mathrm{Pd}\left(\mathrm{PPh}_{3}\right)_{4}\right]$ and $\mathrm{N}$-benzhydryl amine, all of them in catalytic amounts, afforded the formation of the expected allyl products in high yields and excellent enantioselectivities. The reaction ${ }_{65}$ pathway is very similar to the original catalytic system except that the $\eta^{3}$-allyl-Pd-complex is formed upon activation of the allylic alcohol by the chiral phosphoric acid and the enamine is formed in situ by 


\section{Cite this: DOI: 10.1039/c0xx00000x}

\section{www.rsc.org/xxXXXX}

ARTICLE TYPE

(a)

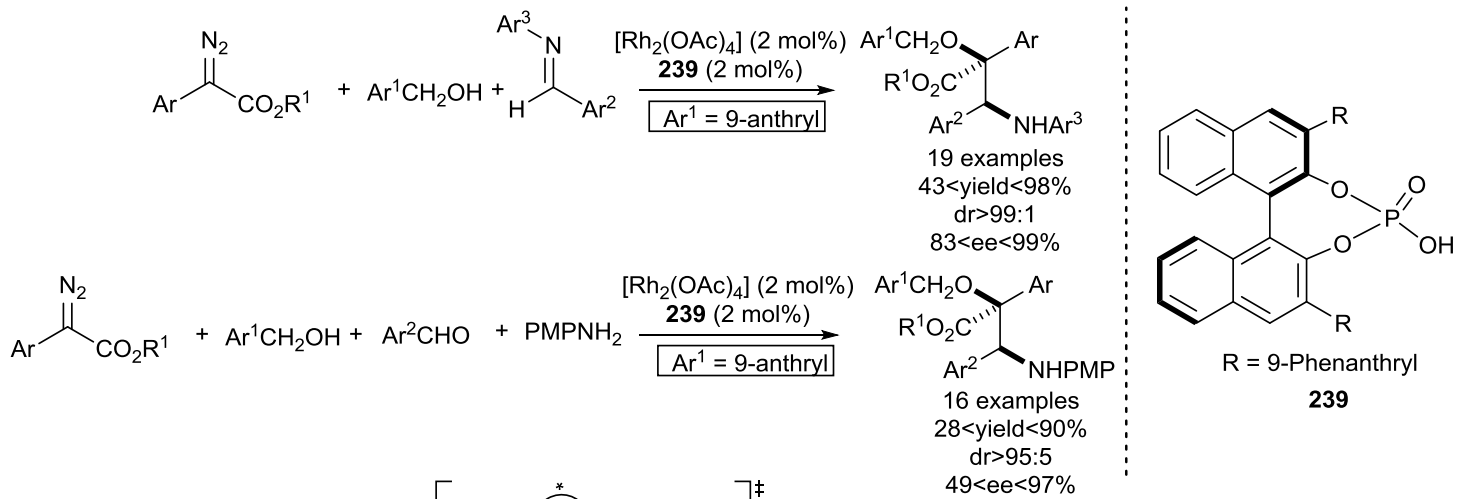

(b)

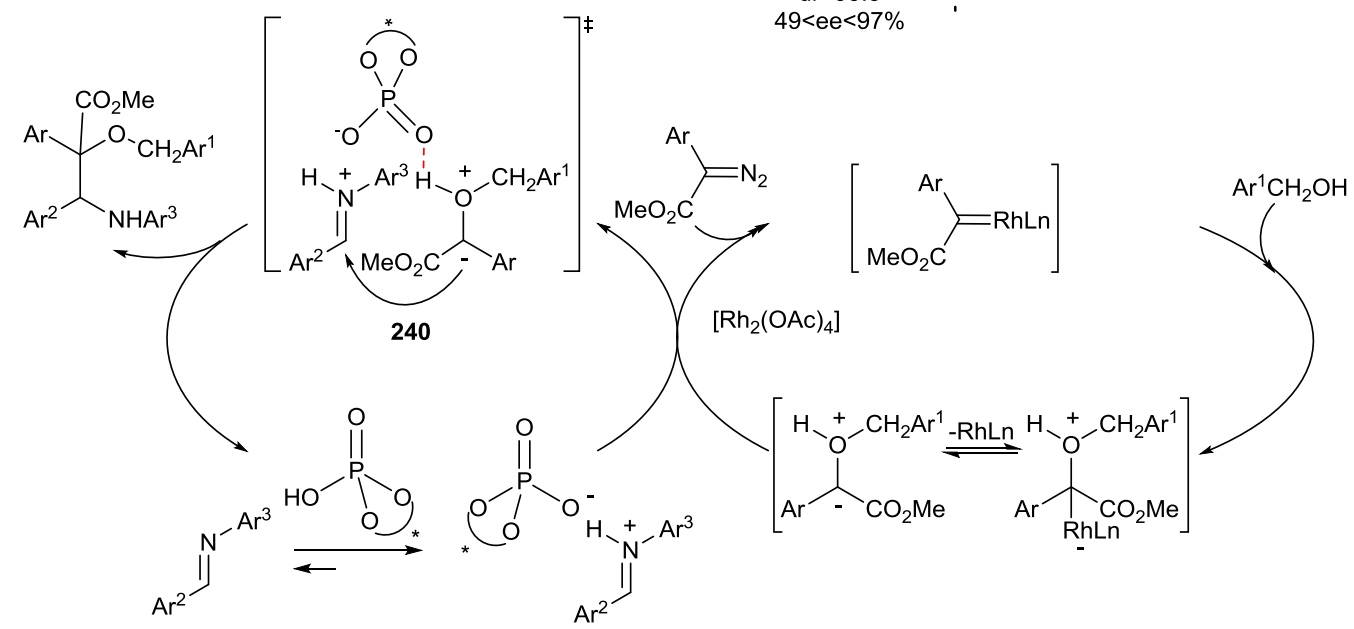

Fig. 68 Multi-component Mannich-type reactions involving the cooperative action of an achiral Rh catalyst and a chiral phosphoric acid. (a) Three- or four-component reactions are selectively catalysed by a mixture of $\left[\mathrm{Rh}_{2}(\mathrm{OAc})_{2}\right]$ and the chiral phosphoric acid 239. (b) Proposed catalytic cycle for the three-component reaction. PMP = $p$-methoxyphenyl. Refs: see the text.

5 reaction between the aldehyde and the $N$-benzhydryl amine. Accordingly, the same key catalytic species (i.e. 238, Fig. 67) at the origin of the high degree of stereocontrol is postulated.

Interestingly, transitions states involving a bridging chiral phosphate anion between an activated iminium substrate and a 10 transition-metal complex has been postulated for other catalytic reactions. ${ }^{484} \mathrm{Hu}$ and co-workers developed multi-component Mannich-type reactions, the key step being the selective trapping of oxonium ${ }^{594,595}$ or carbamate ${ }^{596}$ ylides with chiral iminium salts. In their initial catalytic protocol, the combination of $\left[\mathrm{Rh}_{2}(\mathrm{OAc})_{4}\right]$ 15 and a chiral phosphoric acid catalysed the asymmetric threecomponent Mannich-type reactions involving a diazo compound, a benzylic alcohol and an imine (Fig. 68, a). ${ }^{594}$ The strategy constitutes an attractive synthetic approach towards the preparation of enantiopure syn- $\beta$-amino- $\alpha$-hydroxyl acid 20 derivatives. The imine can also be generated in situ from the condensation between an aldehyde and an amine, rendering a fourcomponent reaction. ${ }^{595}$ The diastereoselectivities and enantioselectivities are high only when a bulky alcohol (9anthrylbenzylic alcohol) is used. In the proposed catalytic cycle ${ }_{25}$ (Fig. 68, b), decomposition of the diazo compound with
$\left[\mathrm{Rh}_{2}(\mathrm{OAc})_{4}\right]$ yields a rhodium carbenoid that further inserts into the $\mathrm{O}-\mathrm{H}$ bond of the bulky alcohol, generating a reacting ylide intermediate. Reaction of the chiral phosphoric acid 239 with the imine substrate independently gives the activated iminium salt. 30 Following a stepwise process, the transient oxonium ylide is trapped by the chiral iminium salt yielding the desired product in high yield and good to excellent de and ee values. In the proposed transition state the phosphate anion bridges the ylide and the iminium ion (240, Fig. 68, b). The selectivity of the reaction is 35 probably mediated by this well-organized ternary complex. A similar multi-component reaction was investigated that replaced the previously used alcohol by a carbamate (Fig. 69, a). ${ }^{596}$ The insertion of the rhodium carbenoid into the $\mathrm{N}-\mathrm{H}$ bond of the carbamate generates a protic ammonium ylide. The insertion is 40 stepwise allowing the transient ylide to be trapped by an iminium phosphate (see 243, Fig. 69, a). The presence of a phosphoric acid is primordial here in order both to activate the iminium substrate and to minimize the 1,2-proton shift side reaction. While screening a series of chiral phosphoric acids, the authors observed that the 45 nature of the 3,3'-substituents of the binaphthyl group strongly influences the diastereoselectivity of the reaction. 


\section{Cite this: DOI: $10.1039 / \mathrm{c0xx00000x}$}

(a)

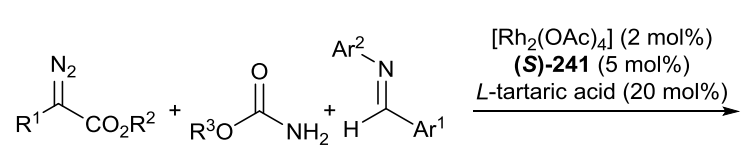

(b)

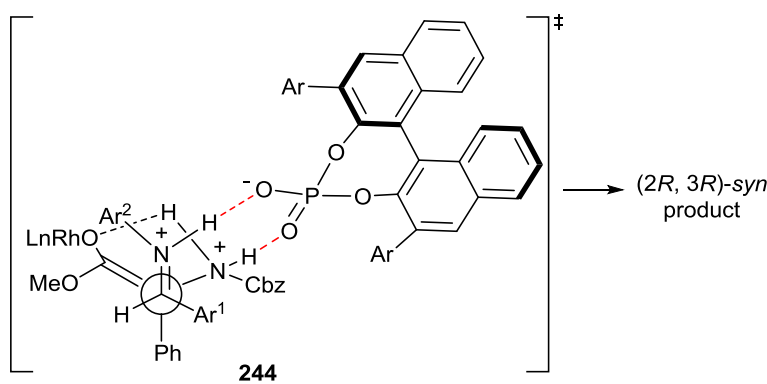

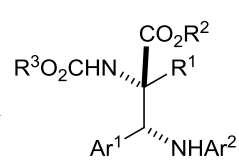

$(2 R, 3 R)$-anti 20 examples $66<$ yield $<93 \%$
$\mathrm{dr}>75: 25$ $89<$ ee $<99 \%$<smiles>[R]C(=[W])O[R20]C([NH3+])=O</smiles>
$\left[\mathrm{Rh}_{2}(\mathrm{OAc})_{4}\right](2 \mathrm{~mol} \%)$ (R)-242 (5 mol\%) L-tartaric acid (20 mol\%)

$$
\mathrm{R}^{1} \mathrm{CO}_{2} \mathrm{R}^{2}{ }_{\mathrm{R}^{3} \mathrm{O}}{ }_{\mathrm{NH}_{2}}^{+}{ }_{\mathrm{Ar}^{1}}
$$

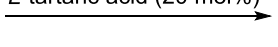

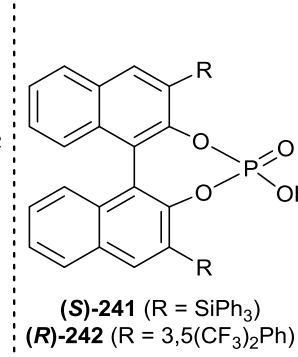

R) $-242\left(\mathrm{R}=3,5\left(\mathrm{CF}_{3}\right)_{2} \mathrm{Ph}\right)$

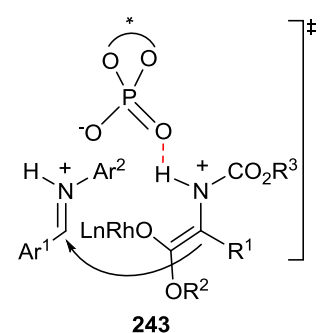

243 $(2 R, 3 S)$-syn 9 examples $67<$ yield $<90 \%$ $\mathrm{dr}>75: 25$ $88<$ ee $<99 \%$
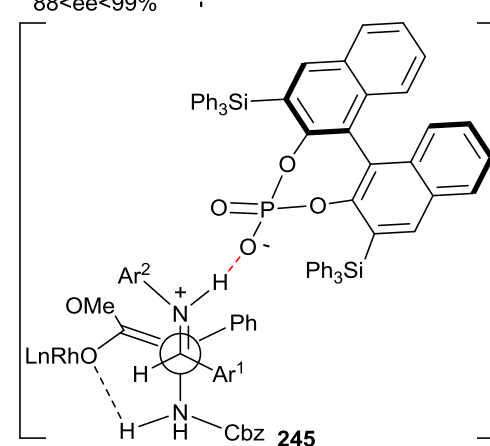

Fig. 69 Mannich-type reactions involving a diazo compound, a carbamate and an imine catalysed by a combination of an achiral Rh catalyst and a chiral phosphoric acid. (a) The diastereoselectivity of the reaction depends on the nature of the chiral phosphoric acid employed. A proposed transition state for 5 the reaction: the transient ylide is trapped by the iminium, the phosphate anion acts as a bridge between the two reaction partners. (b) Representation of the different coordination modes of the phosphate anion that may be at the origin of the opposite selectivity observed with $(\boldsymbol{S})-\mathbf{2 4 1}$ compared to $(\boldsymbol{R})$-242. Refs: see the text.

The bulky phosphoric acid $(\boldsymbol{S})$-241 yields the anti diastereoisomer while $(\boldsymbol{R})-\mathbf{2 4 2}$ led to opposite diastereoisomer. Based on these 10 observations, the authors proposed two different transition states depending on the nature of the phosphoric acid involved in the reaction. In the case of $(\boldsymbol{R}) \mathbf{- 2 4 2}$, the phosphoric acid interacts with both the iminium and the rhodium ylide favouring the syn-addition of the cis-enolate (the ammonium ylide) to the iminium ion (244,

15 Fig. 69, b). In contrast, chiral phosphoric (S)-241 can only bind the iminium ion and the resulting open-chain anti-TS may give rise to the opposite diastereoisomer (245, Fig. 69, b).

A combination of metal catalyst and a chiral acid also proved to be powerful for the asymmetric alkynylation of imines, but the 20 involvement of a bridging chiral base, as mentioned above for the phosphate anion, was not postulated. ${ }^{597,598}$

The metal-catalysed asymmetric reduction of imine with hydrogen gas can be performed by mixing an achiral metal complex and a chiral Brønsted acid. In 2011, Beller and co25 workers screened various metal catalyst/chiral phosphoric acid combinations for the hydrogenation of $\mathrm{N}$-(1phenylethylidene)aniline 246 (Fig. 70 left). ${ }^{599}$ A combination of the Knölker iron complex 145 (Fig. 36) and (S)-Trip provided the respective amine product in $81 \%$ yield and $94 \%$ ee at $65^{\circ} \mathrm{C}$. Upon 30 mixing Trip, the Knölker iron complex and the imine substrate, the authors isolated the iron-amine complex 247 and its formation during the hydrogenation process was also confirmed. Although this catalytic intermediate likely results from the reaction between the iminium phosphate and the Knölker complex, the respective 35 transition state has not been postulated. Based upon the previous examples and mechanistic studies on related reactions made very recently by Xiao, ${ }^{72}$ we propose a ternary complex with a bridging phosphate anion between the iminium ion and the Knölker complex (248, Fig. 70 right).

40 In 2008, Xiao and co-workers found that a mixture of a chiral $\mathrm{Ir}^{\mathrm{III}}$-diamine complex (related to [IrCp*(TsDPEN)]) and a chiral phosphoric acid was a selective catalytic system for the hydrogenation of acyclic imines. ${ }^{600}$ The iridium complex alone is inactive whereas chiral $\left[\mathrm{IrCp} *(\mathrm{TsDPEN})\left(\mathrm{H}_{2} \mathrm{O}\right)\right]\left[\mathrm{SbF}_{6}\right]$ is poorly 45 selective. The iridium phosphate complex 249, formed in situ by protonation of the neutral complex with the chiral phosphoric acid, is selective for the reaction (Fig. 71, a). However, additional chiral 


\section{Cite this: DOI: $10.1039 / \mathrm{c0xx} 00000 x$}

\section{www.rsc.org/xxxxxx}

ARTICLE TYPE

phosphoric acid is needed to increase the activity. Again

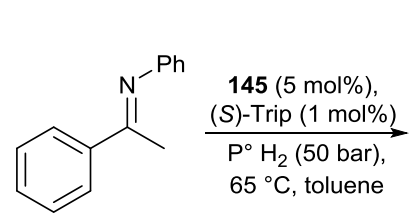

246<smiles>CC(Nc1ccccc1)c1ccccc1</smiles>

$81 \%$ yield, $94 \%$ ee

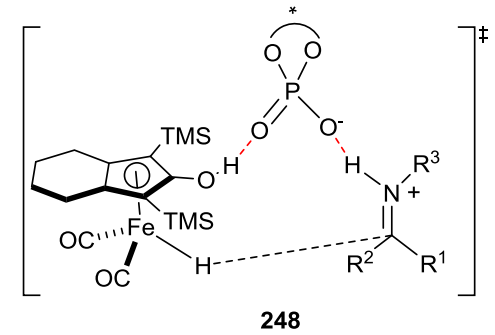

248

Fig. 70 Achiral metal catalyst and chiral phosphoric acid as a mixed catalytic system for the selective hydrogenation of imines. Left: Knölker iron complex 145 and $(S)$-Trip for the asymmetric hydrogenation of imine substrates. Middle: iron-amine complex 247 observed as an intermediate of the catalytic reaction. Right: Proposed transition state 248 involving 145, the $(S)$-Trip anion and the iminium ion. Ref: see the text.

(a)

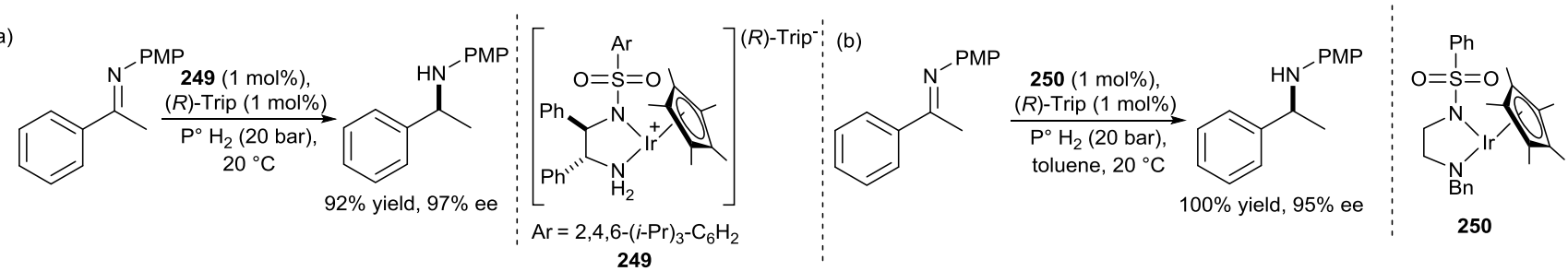

(c)

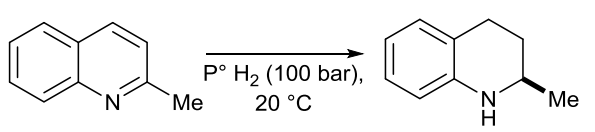

with $251(1 \mathrm{~mol} \%)+252(1 \mathrm{~mol} \%)$, conv. $60 \%$, ee $-38 \%$ with $254(1 \mathrm{~mol} \%)+253(1 \mathrm{~mol} \%)$, conv. $>95 \%$, ee $82 \%$

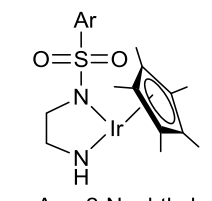

$\mathrm{Ar}=2$-Naphthyl

251

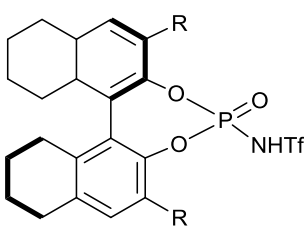

$\mathrm{R}=2,4,6-(i-\mathrm{Pr})_{3}-\mathrm{C}_{6} \mathrm{H}_{2}, 252$

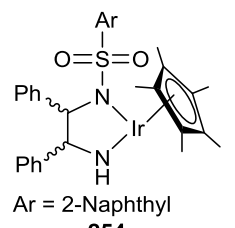

254

(d)

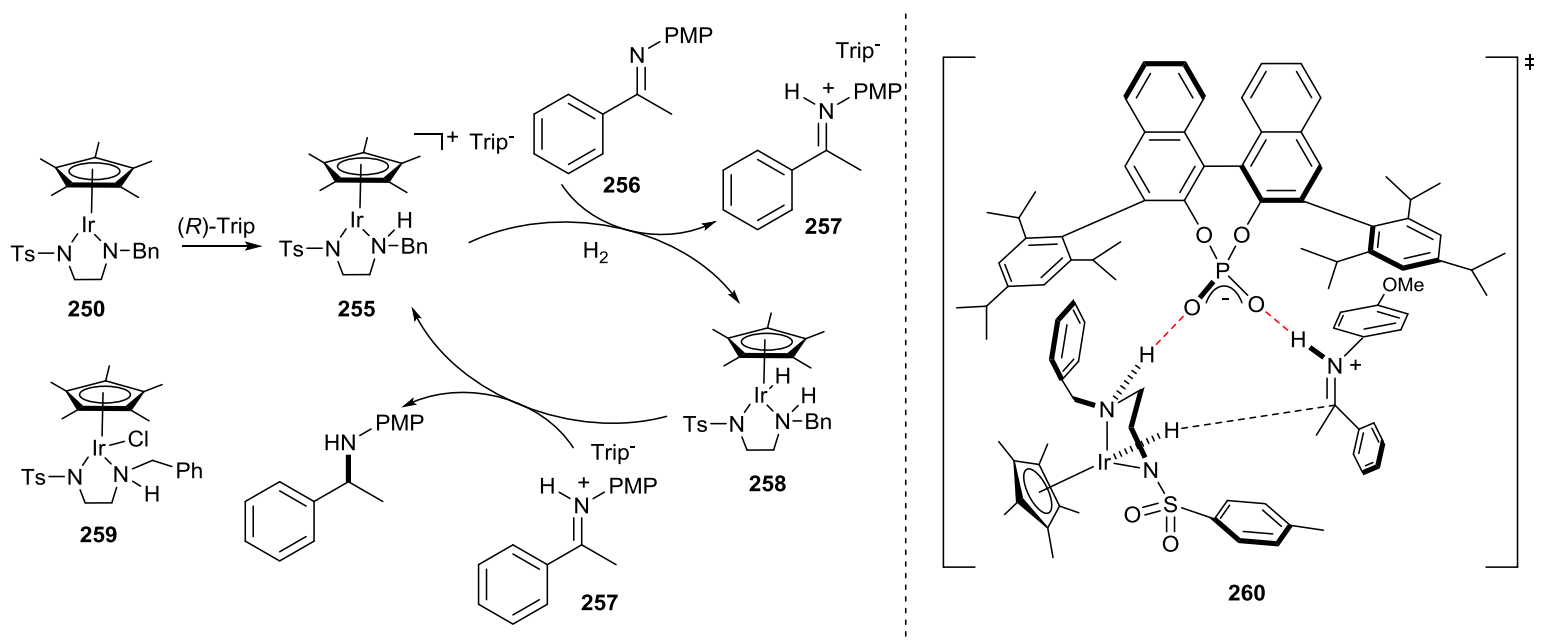

Fig. 71 Asymmetric hydrogenation of imine substrates with a combination of achiral/racemic Ir-diamine complex and a chiral Brønsted acid. (a) Combination of a chiral Ir-diamine complex and $(R)$-Trip for the hydrogenation of acyclic imines. (b) Combination of an achiral Ir-diamine complex 250 and $(R)$-Trip for the hydrogenation of acyclic imines. (c) Combination of an achiral (251) or a racemic (254) Ir-diamine complex and a chiral $N$ triflylphosphoramide for the hydrogenation of quinaldine. (d) Catalytic cycle for the asymmetric hydrogenation of $\mathbf{2 5 6}$ performed with a combination of $\mathbf{2 5 0}$ and $(R)$-Trip. Representation of the more stable ternary complex $\mathbf{2 6 0}$ that involves the $c i s-\left(S_{\mathrm{Ir}}, S_{\mathrm{N}}\right)$-Ir complex, the $(R)$-Trip anion and the iminium ion. Refs: see the text. 


\section{Cite this: DOI: $10.1039 / \mathrm{c0xx00000x}$}

the Trip anion proved to be the anion of choice and the best combination exhibited selective hydrogenation of acyclic imines with ees up to $98 \%$. Later, Rueping and co-workers performed the 5 asymmetric hydrogenation of quinaldine by mixing an achiral Irdiamine complex and a chiral $\mathrm{N}$-triflylphosphoramide anion. ${ }^{601}$ With ethylenediamine as the diamine ligand (complex 251), a maximum of $38 \%$ ee can be obtained through a small screening of chiral anions (Fig. 71, c). By employing a racemic diamine ligand 10 (derived from TsDPEN, 254), the selectivity is increased up to $82 \%$ ee with 253 as the chiral phosphoric acid. The combination proved successful for the asymmetric hydrogenation of various 2'substituted quinolines. Based upon the observation that $(R, R)-\mathbf{2 5 4}$ is more active than $(S, S)$-254, the selectivity likely arose from the 15 kinetic discrimination of the two enantiomeric forms of the racemic complex by the chiral Brønsted acid.

Xiao and co-workers finally demonstrated that a high level of selectivity is achievable for the hydrogenation of acyclic imines by simply combining an achiral iridium complex 250 and $(R)$-Trip 20 (Fig. 71, b). ${ }^{72}$ An in-depth mechanistic study of the reaction enables the observation of catalytic intermediates and the proposition of a transition state. The catalytic cycle is presented in Fig. 71, d: i) the first step is the generation of the cationic phosphate iridium species $\mathbf{2 5 5}$ by protonation of the inactive 25 neutral Ir ${ }^{\mathrm{III}}$ complex $\mathbf{2 5 0}$, ii) this cationic intermediate $\mathbf{2 5 5}$ reacts with dihydrogen and the imine substrate $\mathbf{2 5 6}$ to form the neutral iridium hydride $\mathbf{2 5 8}$ and the activated iminium phosphate $\mathbf{2 5 7}$ (the reaction probably occurs through the formation of dihydrogen complex that is deprotonated by the imine similarly to step B in 30 Fig. 61), iii) the hydride transfer step yields the amine product and regenerates the cationic iridium complex 255 . The hydride complex 258, intermediate in the postulated catalytic cycle, can be prepared independently or isolated from the catalytic conditions as a mixture of diastereoisomers (chirality located on the Ir atom and 35 on the nitrogen atom of the amino ligand). Using racemic 258 for the stoichiometric hydrogenation of the chiral iminium phosphate salt yields the amine product with the same ee as the one obtained under catalytic conditions. This indicates that the asymmetric induction arises in the hydride transfer step rather than from the 40 enantioselective formation of $\mathbf{2 5 8}$. The formation of a hydrogenbonded complex involving the iridium hydride complex, the iminium ion and the $(R)$-Trip anion is strongly supported by NMR spectroscopy. Finally, more spectroscopic data have been accumulated using $\mathbf{2 5 9}$ as a non-reactive iridium model complex, 45 imine 256 and $(R)$-Trip. Based upon the observation of key NOE signals, a series of structures has been generated using molecular mechanics and the most popular ones that satisfied the NOE signals were optimised by DFT. This led to two plausible ternary structures cis- $\left(S_{\mathrm{Ir}}, S_{\mathrm{N}}\right)-\mathrm{Ir}$ and trans- $\left(R_{\mathrm{Ir}}, S_{\mathrm{N}}\right)$-Ir that share common 50 features: i) the chiral phosphate anion acts as a bridge between the iridium hydride complex and the iminium ion and, ii) besides the hydrogen bonds established between the $\mathrm{N}-\mathrm{H}$ proton of the iminium ion and one oxygen atom of the Trip anion and between the $\mathrm{N}-\mathrm{H}$ proton of the diamine ligand and one oxygen atom of the 55 Trip anion, a range of $\mathrm{C}-\mathrm{H}-\pi$ interactions has been identified. The difference between the two calculated transitions state structures arises from the presence of two additional $\mathrm{C}-\mathrm{H}-\pi$ interactions detected in the $c i s-\left(S_{\mathrm{Ir}}, S_{\mathrm{N}}\right)-\mathrm{Ir}$ transition state structure that may explain its higher stability. The hydride analogue of this Ir catalyst 60 - iminium ion - Trip anion ternary complex would afford the experimentally-observed $S$-configured amine. These results strongly support the formation of a well-organised transition state involving the metal complex, the iminium ion and the phosphate anion each of them interacting through a combination of hydrogen 65 bond interactions and $\mathrm{C}-\mathrm{H}-\pi$ interactions (260, Fig. 71). This selfassembled ternary complex is primordial for selectivity, at the cost of a lower reaction rate compared to hydrogenation reactions performed with non hydrogen-bonding counteranions.

These examples demonstrated that cooperative catalysis 70 between a metal catalyst and an organocatalyst may be the result of several attractive interactions between the different reaction partners involved in the selectivity-determining step. It is interesting to note that in most of the transition states mentioned above, a phosphate anion serves as a hydrogen-bonded chiral relay 75 between the metal complex and the activated substrate. The strategy is useful and will probably be extended to catalytic reactions that cannot be accomplished by means of classical catalysts.

\subsection{Organocatalysts - substrate interactions}

80 We already presented in section 5.2.2.1 examples of bifunctional organocatalysts that are formed through the selfassembly of two monofunctional modules. Jacobsen and coworkers have reported a catalytic process that possesses a higher degree of complexity: the substrate, an anion and a bifunctional 85 organocatalyst form a highly-organised self-assembly through several types of non-covalent interactions. The example followed the anion-binding strategy $y^{5,52}$ that operates through the binding of an anion by thiourea or urea catalysts (see one example in Fig. 49) but the very precise investigation of the reaction pathway and of 90 the selectivity-determining transition structure deserves particular attention.

The formal [4+2] cycloaddition of $N$-aryl imines and electron rich olefins is known as the Povarov reaction. In the presence of an acid (e.g. triflic acid), the reaction proceeds quickly but is not 95 selective. Jacobsen and co-workers found that the combination of the chiral bifunctional sulfinamido urea derivative $\mathbf{2 6 5}$ and orthonitrobenzenesulfonic acid (NBSA) gave the exo adduct of the model reaction in high diastereo- and enantioselectivity (up to $91 \%$ ee, Fig. 72). ${ }^{476} \mathrm{~A}$ series of control experiments indicate a 100 cooperative role of the urea and sulfinamide groups of $\mathbf{2 6 5}$ in the rate- and enantioselectivity-determining steps of the catalytic reaction. The reaction catalysed by a combination of 265 and 


\section{Cite this: DOI: $10.1039 / \mathrm{c0xx00000x}$}

NBSA has a large scope and provides tetrahydroquinoline derivatives, biologically-relevant synthons, with high degree of optical purity.

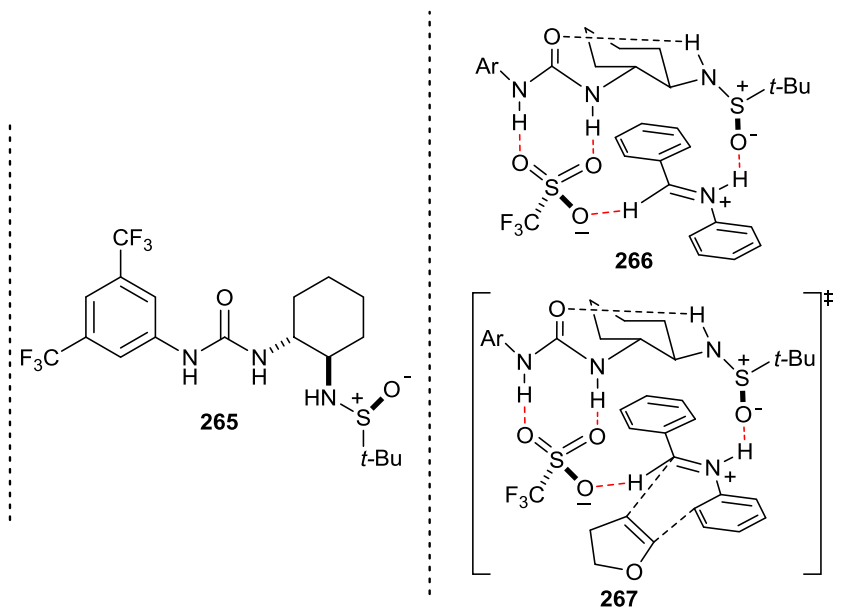

5 Fig. 72 Cooperative activation of the imine substrate by a chiral bifunctional catalyst and an achiral anion. Left: asymmetric Povarov reaction catalysed by a combination of the bifunctional catalyst $\mathbf{2 6 5}$ and NBSA. Right: more stable structure $\mathbf{2 6 6}$ found for the 265-OTf-iminium ternary complex and proposed transition state 267 for the Povarov reaction between 261 and 262 catalysed by a mixture of 265 and triflic acid. Ref: see the text.

The authors first investigated the nature of the interaction between the iminium salt, formed in situ by the acid-base reaction 10 between the imine and the triflic acid, and the bifunctional catalyst 265. They first compared the chemical shift of the formyl proton of the iminium salt upon complexation by $\mathbf{2 6 5}$ and an achiral mono-urea lacking the sulfinamide group. They found that in the first case the signal is slightly upfield shifted compared to the free 15 iminium salt whereas in the second case the signal is highly downfield shifted. They proposed that the iminium-OTf ion pair is tighter in the presence of $\mathbf{2 6 5}$ and this was further confirmed by DFT calculations focused on the 265-OTf-iminium ternary complex. In the four energy-minimized structures that have been 20 located both the $\mathrm{N}-\mathrm{H}$ and the $\mathrm{C}-\mathrm{H}$ protons of the iminium ion are involved in hydrogen bond interactions with either the triflate anion or the $\mathrm{S}-\mathrm{O}$ bond of the sulfinamide group. The more stable structure is represented in Fig. 72 (266). Kinetic studies reveal that the presence of the additional interaction between the formyl group 25 of the iminium and the catalyst-sulfinamide group decreases the rate of the reaction. The high value of the bonding constant found between the triflate iminium salt and $265\left(\mathrm{~K}=9000 \pm 2000\right.$ L.mol$^{-}$ $\left.{ }^{1}\right)$ explains why the reaction pathway that involves the 265-OTfiminium ternary complex is predominant during the catalytic

30 reaction preventing the unselective pathway (reaction of the uncomplexed-iminium) to occur. The selectivity-determining step of the reaction is the concerted [4+2] cycloaddition and several possible transition structures have been modeled using DFT or $a b$ initio methods. In the lowest-energy structure that leads to the 35 experimentally-observed $(R)$-enantiomer $\mathbf{2 6 3}$, the hydrogen bond interactions between the formyl $\mathrm{C}-\mathrm{H}$ proton of the iminium ion and the sulfinamide group and between the $\mathrm{N}-\mathrm{H}$ proton of the iminium ion and the triflate anion are still present. In addition, a $\pi$ - $\pi$ interaction between the $N$-aryl group of the iminium ion and the 40 aryl group hold by the urea function of $\mathbf{2 6 5}$ has also been identified. This interaction is absent in the transition structure that leads to the minor enantiomer. These observations demonstrated that the selectivity observed in this acid-catalysed Povarov reaction arose from a combination of specific hydrogen bond interactions and $\pi$ ${ }_{45} \pi$ interaction between the iminium ion, the anion and the bifunctional catalyst present in the transition structure (267, Fig. 72). The hydrogen bond interactions were already present in $\mathbf{2 6 6}$ placing the reactive iminium ion in an ideal position for the attack of the electron-rich olefin $\mathbf{2 6 2}$. $^{602,603}$

50 The structure of the transition state that has been established through a combination of experimental and computational experiments, presents some similarity with the well-organised transition state structures presented above for the metal catalyst anion or metal catalyst - organocatalyst cooperative systems. ${ }_{55}$ Obviously, the design of multi-component catalytic systems that can self-assemble by means of specific interactions will gain interest in the future, mostly in the course of enantioselective reactions that cannot been performed with simple catalytic systems.

\section{${ }_{60}$ 7. Critical analysis and perspectives}

As regards the area of "supramolecular catalysis beyond enzyme mimics", one can wonder whether a comprehensive review is achievable for such a broad and interdisciplinary research domain. In spite of the 600 references quoted here, the answer is certainly 65 no. To the best of our knowledge, we provided all the references dealing with the design of non-covalent interactions between two ligands (intra- and intermolecular) or between a ligand and a complementary ligand additive. It was facilitated by the relative 
novelty of these areas: 2003 for ligand - ligand interactions ${ }^{56}$ without taking into consideration the work on SPOs ligands (an avant la lettre example of supramolecular bidentate ligand), 2001 for ligand - ligand additive interaction. ${ }^{57}$ Ligand - substrate 5 interactions have been probed more than 30 years by several Japanese groups, ${ }^{321}$ the ligand design was driven by trial-and-error ligand screening with the expectation that the presence of a functional group in the remote part of the ligand can be beneficial for selective substrate binding and activation. It was also 10 established experimentally that the presence of an $\mathrm{N}-\mathrm{H}$ group in the ligand dramatically increases the efficiency of hydrogenation catalysts. This led to the discovery of the very efficient NoyoriIkariya class of catalysts and the $\mathrm{N}-\mathrm{H}$ effect ${ }^{3}$ was rationalized by the formation of non-covalent ligand - substrate interactions. The 15 number of related catalysts has exploded and the $\mathrm{N}-\mathrm{H}$ effect is now considered as an important design element for hydrogenation catalysts. We have compiled several examples of catalytic systems whose efficiency was ascribed to ligand - substrate interactions. However, the beneficial presence of a functional group in the 20 ligand has probably been found in other catalytic systems for which ligand - substrate interactions were not always suspected. As a consequence, a comprehensive coverage of the area seems unlikely. We already mentioned that most of the privileged organocatalysts developed to date can be considered in essence as 25 supramolecular. We focused here on a small part of supramolecular organocatalysts, the modularly designed organocatalysts (MDOs), because we believe that the analogies that exist with supramolecular metal catalysis can stimulate both fields of research. Again, this area is expanding quickly and our 30 idea was to point out the more promising results rather than to give a full coverage of the area. The same statement can be made for the last section, notably for the chiral counteranion-directed catalysis strategy that is currently an intense topic of research. ${ }^{5,53}$ Despite an assumed degree of incompleteness, we can establish 35 general trends and propose perspectives for the different approaches and strategies described in this review.

Intra ligand - ligand interaction: the area raised a fundamental question: can ligand-metal binding and ligand - ligand interactions co-exist? A plethora of supramolecular bidentate ligands have now 40 been disclosed demonstrating the success of the approach. The type of the complementary units and the nature of the reactions investigated differ from one catalytic system to another. On a quantitative point of view, it seems that the area expands exponentially from 2003 to 2008 but the number of publications 45 seems to steadily decrease from that time onwards. Clearly, a revitalization of the area is necessary after having demonstrated the proof-of-concept on simple catalytic reactions. Designing bidentate supramolecular ligands has proven to be successful for the building and screening of catalyst libraries mostly in ${ }_{50}$ hydrogenation and hydroformylation reactions. However, the approach is complementary to the screening of mixtures of monodentate ligands, which has also proven to be successful in the same or related catalytic reactions. ${ }^{604}$ In both approaches, the catalytic performance relies upon the formation of a ${ }_{55}$ heterocombination of ligands that is more active and selective than the respective homocombinations. With well-chosen complementary interactions, the respective hetero-complexes can be formed exclusively. When the activity of the homo-complexes is low, or their selectivity still acceptable, $100 \%$ formation of 60 hetero-complex may not be primordial. From the mechanistic viewpoint, whether the ligand - ligand interactions are maintained during the whole catalytic system needs to be ascertained in several cases. Mild catalytic conditions (low temperature and pressure, absence of base, apolar solvent) are usually employed that might ${ }_{65}$ prevent the dissociation of the ligand - ligand interactions. This has been demonstrated through a series of experimental and computational studies in rhodium-catalysed hydroformylation of linear alkenes performed by a hydrogen-bonded ligand. ${ }^{136}$ For a given self-assembled ligand, it is important to note that a variation 70 of the catalytic conditions can totally change the mode of action of the respective catalyst. In a few cases, the ligand - ligand interactions, which have been observed in the precatalyst, are lost at the expense of ligand - substrate interactions that are crucial for mediating the selectivity of the reaction. ${ }^{73,134,135,142}$ Well-chosen 75 control experiments are needed to ascertain the nature of the selectivity-determining interaction; a crucial parameter in the aim of designing improved catalytic systems. One wish for the future remains the discovery of new reactions and new applications, because as we already mentioned, the supramolecular "machinery" 80 has hardly been put to practice into this direction. To this end, we consider three promising directions for intramolecular selfassembled ligands: i) developing catalytic systems for which the selectivity arises from the interplay of non-covalent interactions between ligands and remote chiral centres. It will participate to the 85 growth of the so-called supramolecular chirogenesis: a field that is of fundamental importance to understand chirality transfer phenomena occurring in Nature. It can also expand the number of efficient privileged catalysts beyond the usually employed BINOL- or TADDOL-derived phosphane ligands. ii) designing 90 self-assembled ligands that encapsulate their coordinating metal; confinement of metal catalyst can be a solution towards the finding of new catalytic reactions. iii) expanding the number of selfassembled ligands that can operate in water because a reaction in this medium is desirable, whatever the complexity of the catalytic 95 system.

Inter ligand - ligand interaction: Hydrogen bond interactions between metal-salen or metal-porphyrin monomers led to the formation of di- or polynuclear catalysts that have been applied mainly in reactions that involve the formation or the transformation 100 of epoxides. Although the strategy holds promise for catalytic reactions that require the presence of two metal centres in close proximity during the selectivity-determining step, its relevance compared to classical catalysts remains to be fully demonstrated. We also mention in this section examples of photocatalysts which 105 combine one or several metal photosensitizers and one metal catalytic centre embedded in the same supramolecule. Among the plethora of photocatalytic systems that have been reported only a few can be formed in situ through the self-assembly of various building blocks. It is surprising because this approach will enable 110 rapid screening of photosensitizer - catalyst combinations in very valuable processes such as light-driven water splitting reactions. ${ }^{212,218}$

Ligand - ligand additive interaction: although the proof-of-concept has been published in $2001,{ }^{57}$ tuning the 115 properties of a metal complex by means of non-covalent interactions between one of its ligand and a non-catalytic additive 
remains a largely unexplored strategy. Following this keystone report, most of the existing systems are based upon Lewis acid Lewis base interaction between the nitrogen atom of a pyridyl fragment and the metal atom of a metal-porphyrin or metal-salen 5 platform (ligand - template strategy). In few cases unusual selectivity has been observed for known reactions as a probable result of metal encapsulation by the self-assembled ligands; the steric constraint exerted by the capsule limits the conformational freedom of the substrate or the metal-bound intermediates during 10 the selectivity-determining step. Recent examples by the groups of Reek $^{284}$ and $\mathrm{Ooi}^{270,271}$ pointed out the possibility of transferring the chirality from a chiral module to an achiral ligand by means of a combination of hydrogen bond and electrostatic interactions between these two components. The approach seems very 15 promising in order to modulate the electronic and steric properties as well as the chiral environment of the metal catalyst. Further work is needed to expand this area towards more valuable reactions that can benefit from the presence of highly tunable and reversible interactions between the ligand and a complementary additive.

20 Ligand - substrate interaction: during the last decade, the area gained a lot of interest because the efficiency of many catalytic reactions can be improved as a result of ligand - substrate attractive interactions occurring during the catalytic cycle. As the ligand possesses a functional group, the interaction between this 25 functional group and the respective complementary group of the substrate directs the reactive part of the latter in an ideal position for activation by the metal centre. The functional group may also stabilise key catalytic intermediates as well as the transition state structure by means of non-covalent interactions. A distinction can 30 be made between ligands that interact with the substrate and then undergo an acid-base reaction with it (proton transfer), and ligands that bind reversibly the substrate without reacting. A trial-anderror ligand screening usually reveals the beneficial role of the functional group and control experiments help to rationalize the 35 observed catalytic effect. A degree of design is achievable and computational studies can help to determine the structure of the ligand - substrate assembly as well as the optimal length between the functional group of the ligand and the metal. With that goal in mind, highly-directional hydrogen bond and electrostatic 40 interactions proved to be relevant as demonstrated independently by the groups of Crabtree, ${ }^{441,443}$ Breit, ${ }^{404-407} \mathrm{Bach}^{447-449}$ and Reek. ${ }^{456,457}$ On the one hand, the approach is highly valuable when the bifunctional catalyst is able to transform a class of substrates with a selectivity that differs from the one observed with covalent 45 catalytic systems. It is the case for the hydroformylation catalyst developed by the group of Reek that transforms 2-vinyl benzoic acid into the corresponding linear aldehyde whereas classical catalysts yields the branched aldehyde as the main product. ${ }^{457}$ On the other hand, the scope of bifunctional catalysts is limited to 50 substrates for which the distance between their complementary group and their reactive part spans the distance between the functional group of the ligand and the metal. Even though it is hard to know if further achievements are feasible in this area, an ideal bifunctional catalyst may impose a certain degree of 55 conformational rigidity for selective substrate activation and transition state stabilisation while keeping a certain degree of flexibility and adaptability.

Organocatalyst - substrate interaction: supramolecular organocatalysts will continue to be designed that activate the ${ }_{60}$ substrate(s) or reaction intermediates and stabilise the transition state though several types of secondary interactions. ${ }^{4,5}$ MDOs constitute a suitable approach to access bifunctional organocatalyst libraries and to tune the properties of privileged organocatalysts. Various additives have been investigated that modify significantly 65 the efficiency of a given organocatalyst, acting cooperatively for the activation of the substrate. The exact role of the additive depends on the catalytic system; entropic gain related to a closer proximity of the reactants in the self-assembled catalyst, transition state stabilisation, chirality transfer or more complex mechanistic 70 pathways may be at the origin of the beneficial role exhibited by the additive. MDOs have been developed for various organocatalytic systems whatever their mode of activation (Lewis and Brønsted acids, Brønsted bases, aminocatalysts, etc...) and further achievements are needed in the direction of self-assembled 75 catalysts that stretch the limits associated with current organocatalysis (high catalyst loading, limited reaction and substrate scope).

Multiple interactions: cooperative catalysts exist which activate a substrate simultaneously though the interplay of several non80 covalent interactions. Highly-organised transition state structures can explain the selectivity observed within this class of catalysts. Remarkably, a similar ternary structure composed of a chiral anion acting as a bridge between the protonated substrate and the metal catalyst has been proposed for different catalytic systems and 85 reactions. Elaborating a catalyst that incorporate some elements of design in the aim of stabilising a transition state remains an arduous task giving the elusive nature of this structure, however such approach can stimulate both the fields of metal and organic catalysis.

90 To conclude, the design of supramolecular catalysts beyond enzyme mimics has drawn the attention of researchers belonging to all the disciplines of chemical science. The field has expanded continuously in the last decade, reaching an average 5 publications/month rate for the last 6 years (considering the 95 references quoted in this review). We have seen many examples of supramolecular catalysts that changed completely the selectivity of a reaction and in many instances this was achieved by design. This concerns known reactions which facilitates the design. Confinement of metal catalyst or organocatalyst can be a solution 100 towards the finding of new catalytic reactions. In few cases, nonthermodynamic products were obtained that cannot be obtained by other catalysts or synthetic methods. However, prediction is rather difficult in this area and trial-and-error iterations are probably needed to get the desired catalytic effects. An industrial application 105 would underscore the practical importance of supramolecular catalysis. This is not an easy target. Any supramolecular ligand system will be more expensive than a routine ligand such as triphenylphosphine, any multi-component system will be more difficult to be recovered from a substrate and product solution, and 110 thus a new supramolecular catalyst should do something that a simple ligand-metal system cannot. There are examples available of the latter, but apparently the costs do not permit their application yet. Industries have taken patents in this area and thus the chemistry is not only beautiful for academic research but also holds 115 a promise for applications. The elaboration of supramolecular catalysts by design should benefit the parallel emergence of highly- 
efficient computational tools. ${ }^{605}$ Perhaps supramolecular catalysis should focus on what seems most appropriate for this tool, the conversion of more complicated molecules in a highly selective way in a system capable of multiple reactions, as recognition is a 5 key feature.

\section{Notes and references}

${ }^{a}$ Institute of Chemical Research of Catalonia (ICIQ), Av. Països Catalans 16, 43007 Tarragona, Spain.

${ }^{b}$ Current address: Chimie des Polymères, UPMC Université Paris 06 10 and CNRS, UMR 7610, F-75005 Paris, France.

${ }^{c}$ Catalan Institution of Research and Advanced Studies (ICREA), Passeig Lluís Companys 23, 08010 Barcelona, Spain.

\section{Acknowledgement}

We thank MICINN (Grant CTQ2011-28512) and the ICIQ ${ }_{15}$ Foundation for financial support.

\section{References}

1. S. E. Clapham, A. Hadzovic and R. H. Morris, Coord. Chem. Rev., 2004, 248, 2201-2237.

2. T. Ikariya, K. Murata and R. Noyori, Org. Biomol. Chem., 2006, 4, $20 \quad 393-406$.

3. B. Zhao, Z. Han and K. Ding, Angew. Chem. Int. Ed., 2013, 52, 47444788.

4. R. R. Knowles and E. N. Jacobsen, Proc. Natl. Acad. Sci. U. S. A., 2010, 107, 20678-20685.

25 5. K. Brak and E. N. Jacobsen, Angew. Chem. Int. Ed., 2013, 52, 534561.

6. Y. Murakami, J.-I. Kikuchi, Y. Hisaeda and O. Hayashida, Chem. Rev., 1996, 96, 721-758.

7. A. J. Kirby, Angew. Chem. Int. Ed. Engl., 1996, 35, 707-724.

30 8. W. B. Motherwell, M. J. Bingham and Y. Six, Tetrahedron, 2001, 57, $4663-4686$

9. A. J. Kirby and F. Hollfelder, From Enzyme Models to Model Enzymes, RSC Publishing, Cambridge, 2009.

10. Z. Y. Dong, Q. Luo and J. Q. Liu, Chem. Soc. Rev., 2012, 41, 78907908.

11. M. Raynal, P. Ballester, A. Vidal-Ferran and P. W. N. M. van Leeuwen, Chem. Soc. Rev., 2013, Part 2.

12. I. Tabushi, Acc. Chem. Res., 1982, 15, 66-72.

13. R. Breslow and S. D. Dong, Chem. Rev., 1998, 98, 1997-2011.

40 14. D. M. Vriezema, M. C. Aragones, J. A. A. W. Elemans, J. J. L. M. Cornelissen, A. E. Rowan and R. J. M. Nolte, Chem. Rev., 2005, 105, 1445-1489.

15. M. D. Pluth, R. G. Bergman and K. N. Raymond, Acc. Chem. Res., 2009, 42, 1650-1659.

45 16. M. Yoshizawa, J. K. Klosterman and M. Fujita, Angew. Chem. Int. Ed., 2009, 48, 3418-3438.

17. S. Otto and J. B. F. N. Engberts, Org. Biomol. Chem., 2003, 1, 28092820.

18. R. Breslow, J. Phys. Org. Chem., 2006, 19, 813-822.

50 19. E. A. Wintner, M. M. Conn and J. Rebek, Jr., Acc. Chem. Res., 1994, 27, 198-203.

20. B. G. Bag and G. von Kiedrowski, Pure Appl. Chem., 1996, 68, $2145-$ 2152.

21. D. H. Lee, K. Severin and M. R. Ghadiri, Curr. Opin. Chem. Biol., 55 1997, 1, 491-496.

22. A. Robertson, A. J. Sinclair and D. Philp, Chem. Soc. Rev., 2000, 29, 141-152.

23. N. Paul and G. F. Joyce, Curr. Opin. Chem. Biol., 2004, 8, 634-639.

24. A. Vidonne and D. Philp, Eur. J. Org. Chem., 2009, 593-610.

60 25. V. del Amo and D. Philp, Chem. Eur. J., 2010, 16, 13304-13318.

26. N. Giuseppone, Acc. Chem. Res., 2012, 45, 2178-2188.

27. S. Otto, Acc. Chem. Res., 2012, 45, 2200-2210.

28. J. Rebek, Jr., Acc. Chem. Res., 1984, 17, 258-264.

29. L. Kovbasyuk and R. Krämer, Chem. Rev., 2004, 104, 3161-3187.
65 30. M. J. Wiester, P. A. Ulmann and C. A. Mirkin, Angew. Chem. Int. Ed., 2011, 50, 114-137.

31. C. M. Thomas and T. R. Ward, Chem. Soc. Rev., 2005, 34, 337-346.

32. A. J. Boersma, R. P. Megens, B. L. Feringa and G. Roelfes, Chem. Soc. Rev., 2010, 39, 2083-2092.

70 33. T. R. Ward, Acc. Chem. Res., 2011, 44, 47-57.

34. P. J. Deuss, R. den Heeten, W. Laan and P. C. J. Kamer, Chem. Eur. J., 2011, 17, 4680-4698.

35. M. T. Reetz, Chem. Rec., 2012, 12, 391-406.

36. R. van de Coevering, R. J. M. K. Gebbink and G. van Koten, Prog. 75 Polym. Sci., 2005, 30, 474-490.

37. J. M. Fraile, J. I. García and J. A. Mayoral, Chem. Rev., 2009, 109, 360-417.

38. P. Barbaro and F. Liguori, Chem. Rev., 2009, 109, 515-529.

39. M. J. Wilkinson, P. W. N. M. van Leeuwen and J. N. H. Reek, Org.

80 Biomol. Chem., 2005, 3, 2371-2383.

40. Z. Wang, G. Chen and K. Ding, Chem. Rev., 2009, 109, 322-359.

41. M. Studer, H.-U. Blaser and C. Exner, Adv. Synth. Catal., 2003, 345, 45-65.

42. M. Heitbaum, F. Glorius and I. Escher, Angew. Chem. Int. Ed., 2006, $85 \quad 45,4732-4762$.

43. Y. J. Park, J.-W. Park and C.-H. Jun, Acc. Chem. Res., 2008, 41, 222234.

44. B. Breit, Angew. Chem. Int. Ed., 2005, 44, 6816-6825.

45. A. W. Kleij and J. N. H. Reek, Chem. Eur. J., 2006, 12, 4218-4227.

90 46. T. S. Koblenz, J. Wassenaar and J. N. H. Reek, Chem. Soc. Rev., 2008, 37, 247-262.

47. P. E. Goudriaan, P. W. N. M. van Leeuwen, M.-N. Birkholz and J. N. H. Reek, Eur. J. Inorg.Chem., 2008, 2939-2958.

48. L. A. Joyce, S. H. Shabbir and E. V. Anslyn, Chem. Soc. Rev., 2010, 39, 3621-3632.

49. J. Meeuwissen and J. N. H. Reek, Nature Chem., 2010, 2, 615-621.

50. S. Carboni, C. Gennari, L. Pignataro and U. Piarulli, Dalton Trans., 2011, 40, 4355-4373.

51. R. Bellini, J. I. van der Vlugt and J. N. H. Reek, Isr. J. Chem., 2012, $100 \quad$ 52, 613-629.

52. For a review dealing with the ACDC strategy see: M. Mahlau and B. List, Isr. J. Chem., 2012, 52, 630-638.

53. R. J. Phipps, G. L. Hamilton and F. D. Toste, Nature Chem., 2012, 4 , 603-614.

105 54. P. W. N. M. van Leeuwen, ed., Supramolecular Catalysis, WileyVCH, Weinheim, 2008.

55. Thermodynamic and kinetic data related to non-covalent interactions are out of the scope of this review. However, these parameters will be given if it helps to explain the catalytic behaviour. For the sake of consistency, ionic metal-ligand interaction will be mentioned in the construction of libraries of bidentate ligands but not in the other sections. For a quantitative estimation of non-covalent interactions see the following review: C. A. Hunter, Angew. Chem. Int. Ed., 2004, 43, 5310-5324.

115 56. B. Breit and W. Seiche, J. Am. Chem. Soc., 2003, 125, 6608-6609.

57. V. F. Slagt, J. N. H. Reek, P. C. J. Kamer and P. W. N. M. van Leeuwen, Angew. Chem. Int. Ed., 2001, 40, 4271-4274.

58. T. Ohkuma, H. Ooka, S. Hashiguchi, T. Ikariya and R. Noyori, J. Am. Chem. Soc., 1995, 117, 2675-2676.

120 59. S. Hashiguchi, A. Fujii, J. Takehara, T. Ikariya and R. Noyori, J. Am. Chem. Soc., 1995, 117, 7562-7563.

60. R. Noyori and S. Hashiguchi, Acc. Chem. Res., 1997, 30, 97-102.

61. M. S. Taylor and E. N. Jacobsen, Angew. Chem. Int. Ed., 2006, 45, $1520-1543$.

125 62. T. Akiyama, J. Itoh and K. Fuchibe, Adv. Synth. Catal., 2006, 348, 999-1010.

63. T. Akiyama, Chem. Rev., 2007, 107, 5744-5758.

64. A. G. Doyle and E. N. Jacobsen, Chem. Rev., 2007, 107, 5713-5743.

65. T. Hashimoto and K. Maruoka, Chem. Rev., 2007, 107, 5656-5682.

130 66. T. Ooi and K. Maruoka, Angew. Chem. Int. Ed., 2007, 46, 4222-4266.

67. C. Palomo, M. Oiarbide and R. López, Chem. Soc. Rev., 2009, 38, 632653.

68. X. Fu and C.-H. Tan, Chem. Commun., 2011, 47, 8210-8222.

69. For an instructive comparison between supramolecular and covalent 135 organocatalysts see: P. A. Woods and A. D. Smith, in Supramolecular Chemistry: From Molecules to Nanomaterials, eds. J. W. Steed and P. 
A. Gale, John Wiley \& Sons, Ltd, 2012, DOI: 10.1002/9780470661345.smc9780470661156.

70. For a review on organocatalytic chiral ion pairs in which both anionic and cationic parts are working in a cooperative fashion see: J. F. Briere, S. Oudeyer, V. Dalla and V. Levacher, Chem Soc Rev, 2012, 41, 16961707.

71. A. Macchioni, Chem. Rev., 2005, 105, 2039-2073.

72. W. Tang, S. Johnston, J. A. Iggo, N. G. Berry, M. Phelan, L. Lian, J. Bacsa and J. Xiao, Angew. Chem. Int. Ed., 2013, 52, 1668-1672.

10 73. K. Lang, J. Park and S. Hong, Angew. Chem. Int. Ed., 2012, 51, $1620-$ 1624.

74. P. W. N. M. van Leeuwen, P. C. J. Kamer, J. N. H. Reek and P. Dierkes, Chem. Rev., 2000, 100, 2741-2769.

75. P. C. J. Kamer, P. W. N. M. van Leeuwen and J. N. H. Reek, Acc. Chem. Res., 2001, 34, 895-904.

76. Z. Freixa and P. W. N. M. van Leeuwen, Dalton Trans., 2003, 18901901.

77. Z. Freixa and P. W. N. M. van Leeuwen, Coord. Chem. Rev., 2008, 252, 1755-1786.

20 78. J. Wassenaar and J. N. H. Reek, Org. Biomol. Chem., 2011, 9, 17041713.

79. W. Seiche and B. Breit, in Phosphorus Ligands in Asymmetric Catalysis, ed. A. Börner, Wiley-WCH, Weinheim, 2008, pp. 848-885.

80. P. W. N. M. van Leeuwen and C. F. Roobeek, Hydroformylation of

25 olefins, 1983, Shell Internationale Research Maatschappij B. V., Neth., EP 82576, Chem. Abstr., 99, 121813..

81. W. B. Beaulieu, T. B. Rauchfuss and D. M. Roundhill, Inorg. Chem., 1975, 14, 1732-1734.

82. P. W. N. M. van Leeuwen, C. F. Roobeek, R. L. Wife and J. H. G.

30 Frijns, J. Chem. Soc., Chem. Commun.,, 1986, 31-33.

83. J. L. Cook, C. A. Hunter, C. M. R. Low, A. Perez-Velasco and J. G. Vinter, Angew. Chem. Int. Ed., 2007, 46, 3706-3709.

84. N. V. Dubrovina and A. Börner, Angew. Chem. Int. Ed., 2004, 43, 5883-5886.

35 85. A. Christiansen, C. Li, M. Garland, D. Selent, R. Ludwig, R. Franke and A. Börner, ChemCatChem, 2010, 2, 1278-1285.

86. A. Christiansen, D. Selent, A. Spannenberg, M. Köckerling, H. Reinke, W. Baumann, H. Jiao, R. Franke and A. Börner, Chem. Eur. J., 2011, 17, 2120-2129.

40 87. L. Ackermann, Synthesis, 2006, 1557-1571.

88. L. Ackermann, R. Born, J. H. Spatz, A. Althammer and C. J. Gschrei, Pure Appl. Chem., 2006, 78, 209-214.

89. T. Nemoto and Y. Hamada, Tetrahedron, 2011, 667-687.

90. D. Martin, D. Moraleda, T. Achard, L. Giordano and G. Buono, Chem. Eur. J., 2011, 17, 12729-12740.

91. X.-B. Jiang, A. J. Minnaard, B. Hessen, B. L. Feringa, A. L. L. Duchateau, J. G. O. Andrien, J. A. F. Boogers and A. H. M. de Vries, Org. Lett., 2003, 5, 1503-1506.

92. Q. Jiang, A. J. Minnaard, B. L. Feringa and A. H. M. de Vries, J. Org.

50 Chem., 2004, 69, 2327-2331.

93. X.-B. Jiang, M. van den Berg, A. J. Minnaard, B. L. Feringa and A. H. M. de Vries, Tetrahedron: Asymmetry, 2004, 15, 2223-2229.

94. C. M. Jensen and W. C. Trogler, J. Am. Chem. Soc., 1986, 108, $723-$ 729

55 95. T. Ghaffar and A. W. Parkins, J. Mol. Cat. A: Chem., 2000, 160, 249261.

96. T. Ghaffar and A. W. Parkins, Tetrahedron Lett., 1995, 36, 8657-8660.

97. P. M. Castro, H. Gulyas, J. Benet-Buchholz, C. Bo, Z. Freixa and P. W. N. M. van Leeuwen, Catal. Sci. Technol., 2011, 1, 401-407.

60 98. A. Comas-Vives, G. Ujaque and A. Lledós, Organometallics, 2007, 26, 4135-4144.

99. A. Comas-Vives, G. Ujaque and A. Lledós, THEOCHEM, 2009, 903, $123-132$.

100.F. W. Patureau, M. Kuil, A. J. Sandee and J. N. H. Reek, Angew. Chem. Int. Ed., 2008, 47, 3180-3183.

101.F. W. Patureau, M. A. Siegler, A. L. Spek, A. J. Sandee, S. Jugé, S. Aziz, A. Berkessel and J. N. H. Reek, Eur. J. Inorg. Chem., 2012, 496503.

102.A. M. Kluwer, R. Detz, Z. Abiri, A. M. van der Burg and J. N. H. Reek, 70 Adv. Synth. Catal., 2012, 354, 89-95.

103.P.-A. R. Breuil, F. W. Patureau and J. N. H. Reek, Angew. Chem. Int. Ed., 2009, 48, 2162-2165.
104.J. Meeuwissen, R. Detz, A. J. Sandee, B. de Bruin, M. A. Siegler, A. L. Spek and J. N. H. Reek, Eur. J. Inorg. Chem., 2010, 2992-2997.

75 105.J. Meeuwissen, A. J. Sandee, B. De Bruin, M. A. Siegler, A. L. Spek and J. N. H. Reek, Organometallics, 2010, 29, 2413-2421.

106.P. Štěpnička and I. Císařová, J.Chem. Soc., Dalton Trans., 1998, 28072811.

107.A. M. Trzeciak, P. Štěpnička, E. Mieczyńska and J. J. Ziółkowski, J. Organomet. Chem., 2005, 690, 3260-3267.

108.A. C. Laungani, M. Keller, J. M. Slattery, I. Krossing and B. Breit, Chem. Eur. J., 2009, 15, 10405-10422.

109.W. Seiche, A. Schuschkowski and B. Breit, Adv. Synth. Catal., 2005, 347, 1488-1494.

85 110.B. Breit and W. Seiche, Angew. Chem. Int. Ed., 2005, 44, 1640-1643.

111.C. Waloch, J. Wieland, M. Keller and B. Breit, Angew. Chem. Int. Ed., 2007, 46, 3037-3039.

112.A. C. Laungani, J. M. Slattery, I. Krossing and B. Breit, Chem. Eur. J., 2008, 14, 4488-4502.

90 113.D. Fuchs, G. Rousseau, L. Diab, U. Gellrich and B. Breit, Angew. Chem. Int. Ed., 2012, 51, 2178-2182.

114.M.-N. Birkholz, N. V. Dubrovina, H. Jiao, D. Michalik, J. Holz, R. Paciello, B. Breit and A. Börner, Chem. Eur. J., 2007, 13, 5896-5907.

115.M.-N. Birkholz, N. V. Dubrovina, I. A. Shuklov, J. Holz, R. Paciello,

95 C. Waloch, B. Breit and A. Börner, Tetrahedron: Asymmetry, 2007, 18, 2055-2060.

116.B. Schäffner, J. Holz, S. P. Verevkin and A. Börner, Tetrahedron Lett., 2008, 49, 768-771.

117.I. A. Shuklov, N. V. Dubrovina, E. Barsch, R. Ludwig, D. Michalik and A. Börner, Chem. Commun., 2009, 1535-1537.

118.U. Gellrich, J. Huang, W. Seiche, M. Keller, M. Meuwly and B. Breit, J. Am. Chem. Soc., 2011, 133, 964-975.

119.O. Abillard and B. Breit, Adv. Synth. Catal., 2007, 349, 1891-1895.

120.M. Weis, C. Waloch, W. Seiche and B. Breit, J. Am. Chem. Soc., 2006, 128, 4188-4189.

121.F. Chevallier and B. Breit, Angew. Chem., Int. Ed., 2006, 45, 15991602.

122.T. Šmejkal and B. Breit, Organometallics, 2007, 26, 2461-2464.

123.I. Usui, S. Schmidt, M. Keller and B. Breit, Org. Lett., 2008, 10, $1207-$ $110 \quad 1210$.

124.B. Breit, Pure Appl. Chem., 2008, 80, 855-860.

125.M. de Greef and B. Breit, Angew. Chem., Int. Ed., 2009, 48, 551-554.

126.J. Wieland and B. Breit, Nature Chem., 2010, 2, 832-837.

127.P. A. Duckmanton, A. J. Blake and J. B. Love, Inorg. Chem., 2005, 44, 7708-7710.

128.L. K. Knight, Z. Freixa, P. W. N. M. van Leeuwen and J. N. H. Reek, Organometallics, 2006, 25, 954-960.

129.A. J. Sandee, A. M. van der Burg and J. N. H. Reek, Chem. Commun., 2007, 864-866.

120 130.J. Meeuwissen, M. Kuil, A. M. van der Burg, A. J. Sandee and J. N. H. Reek, Chem. Eur. J., 2009, 125, 10272-10279.

131.M. Durini, E. Russotto, L. Pignataro, O. Reiser and U. Piarulli, Eur. J. Org.Chem., 2012, 5451-5461.

132.Y. Liu, C. A. Sandoval, Y. Yamaguchi, X. Zhang, Z. Wang, K. Kato and K. Ding, J. Am. Chem. Soc., 2006, 128, 14212-14213.

133.A. C. Laungani and B. Breit, Chem. Commun., 2008, 844-846.

134.L. Pignataro, S. Carboni, M. Civera, R. Colombo, U. Piarulli and C. Gennari, Angew. Chem. Int. Ed., 2010, 49, 6633-6637.

135.L. Pignataro, M. Boghi, M. Civera, S. Carboni, U. Piarulli and C. 130 Gennari, Chem. Eur. J., 2012, 18, 1383-1400.

136.U. Gellrich, W. Seiche, M. Keller and B. Breit, Angew. Chem. Int. Ed., 2012, 51, 11033-11038.

137.V. Agabekov, W. Seiche and B. Breit, Chem. Sci., 2013, 4, 2418-2422.

138.Z. Kokan and S. I. Kirin, RSC Advances, 2012, 2, 5729-5737.

135 139.Previously, Gilbertson developed libraries of diphosphine ligands based on a covalent peptide backbone. In some cases, the catalytic selectivity arose from the $\beta$-turn secondary structure adopted by the peptide ligands rather than from the sum of the individual chiral centres. See references 139-141: S. R. Gilbertson, S. E. Collibee and A. Agarkov, J. Am. Chem. Soc., 2000, 122, 6522-6523.

140.S. J. Greenfield, A. Agarkov and S. R. Gilbertson, Org. Lett., 2003, 5, 3069-3072.

141.A. Agarkov, S. Greenfield, D. Kie, R. Pawlick, G. Starkey and S. R. Gilbertson, Biopolymers (Peptides Sci.), 2006, 84, 48-73. 
142.L. Pignataro, C. Bovio, M. Civera, U. Piarulli and C. Gennari, Chem. Eur. J., 2012, 18, 10368-10381.

143.V. F. Slagt, P. W. N. M. van Leeuwen and J. N. H. Reek, Chem. Commun., 2003, 2474-2475.

5 144.M. Kuil, P. E. Goudriaan, P. W. N. M. Van Leeuwen and J. N. H. Reek, Chem. Commun., 2006, 4679-4681.

145.V. F. Slagt, P. W. N. M. van Leeuwen and J. N. H. Reek, Dalton Trans., 2007, 2302-2310.

146.M. Kuil, P. E. Goudriaan, A. W. Kleij, D. M. Tooke, A. L. Spek, P. W.

10 N. M. van Leeuwen and J. N. H. Reek, Dalton Trans., 2007, 23112320.

147.S. Ponsico, H. Gulyas, M. Martinez-Belmonte, E. C. Escudero-Adan, Z. Freixa and P. W. N. M. van Leeuwen, Dalton Trans., 2011, 40, 10686-10697.

15 148.T. Gadzikwa, R. Bellini, H. L. Dekker and J. N. H. Reek, J. Am. Chem. Soc., 2012, 134, 2860-2863.

149.V. F. Slagt, P. W. N. M. van Leeuwen and J. N. H. Reek, Angew. Chem. Int. Ed., 2003, 42, 5619-5623.

150.M. Dieguez, O. Pamies and C. Claver, Tetrahedron Asymmetry, 2004, 15, 2113-2122.

151.Y. J. Yan, Y. X. Chi and X. M. Zhang, Tetrahedron Asymmetry, 2004, 15, 2173-2175.

152.X.-B. Jiang, L. Lefort, P. E. Goudriaan, A. H. M. de Vries, P. W. N. M. van Leeuwen, J. G. de Vries and J. N. H. Reek, Angew. Chem. Int. Ed., 2006, 45, 1223-1227.

153.X.-B. Jiang, P. W. N. M. van Leeuwen and J. N. H. Reek, Chem. Commun., 2007, 2287-2289.

154.P. E. Goudriaan, X.-B. Jang, M. Kuil, R. Lemmens, P. W. N. M. van Leeuwen and J. N. H. Reek, Eur. J. Org. Chem., 2008, 6079-6092.

30 155.P. E. Goudriaan, M. Kuil, X.-B. Jiang, P. W. N. M. van Leeuwen and J. N. H. Reek, Dalton Trans., 2009, 1801-1805.

156.V. F. Slagt, M. Roeder, P. C. J. Kamer, P. W. N. M. van Leeuwen and J. N. H. Reek, J. Am. Chem. Soc., 2004, 126, 4056-4057.

157.J. N. H. Reek, M. Röder, P. E. Goudriaan, P. C. J. Kamer, P. W. N. M.

35 van Leeuwen and V. F. Slagt, J. Organomet. Chem., 2005, 690, 45054516.

158.J. M. Takacs, D. Sahadeva Reddy, S. A. Moteki, D. Wu and H. Palencia, J. Am. Chem. Soc., 2004, 126, 4494-4495.

159.For older examples of bimetallic catalysts that incorporate an

$40 \quad$ assembling metal and a catalytic metal centre see references 159164.F. Senocq, C. Randrianalimanana, A. Thorez, P. Kalck, R. Choukroun and D. Gervais, J. Chem. Soc., Chem. Commun.,, 1984, 1376-1377.

160.R. Choukroun, A. Iraqi, D. Gervais, J.-C. Daran and Y. Jeannin, 45 Organometallics, 1987, 6, 1197-1201.

161.A. M. Trzeciak, J. J. Ziółkowski and R. Choukroun, J. Mol. Cat. A: Chem., 1996, 110, 135-139.

162.N. Wheatley and P. Kalck, Chem. Rev., 1999, 99, 3379-3419.

163.M. Quirmbach, A. Kless, J. Holz, V. Tararov and A. Börner,

50 Tetrahedron: Asymmetry, 1999, 10, 1803-1811.

164.Q. F. Mokuolu, P. A. Duckmanton, P. B. Hitchcock, C. Wilson, A. J. Blake, L. Shukla and J. B. Love, Dalton Trans., 2004, 1960-1970.

165.J. M. Takacs, P. M. Hrvatin, D. S. Reddy and J. L. Clark, New. J. Chem., 2005, 29, 263-265.

55 166.J. M. Atkins, S. A. Moteki, S. G. DiMagno and J. M. Takacs, Org. Lett., 2006, 8, 2759-2762.

167.J. M. Takacs, K. Chaiseeda, S. A. Moteki, D. S. Reddy, D. Wu and K. Chandra, Pure Appl. Chem., 2006, 78, 501-509.

168.N. C. Thacker, S. A. Moteki and J. M. Takacs, ACS Catal., 2012, 2, 2743-2752.

169.S. A. Moteki and J. M. Takacs, Angew. Chem. Int. Ed., 2008, 47, 894897.

170.S. A. Moteki, K. Toyama, Z. Liu, J. Ma, A. E. Holmes and J. M. Takacs, Chem. Commun., 2012, 48, 263-265.

65 171.D. Rivillo, H. Gulyas, J. Benet-Buchholz, E. C. Escudero-Adan, Z. Freixa and P. W. N. M. van Leeuwen, Angew. Chem. Int. Ed., 2007, 46, 7247-7250.

172.D. Rivillo, Universitat Rovira i Virgili, Tarragona, 2010.

173.P. W. N. M. van Leeuwen, D. Rivillo, M. Raynal and Z. Freixa, J. Am. Chem. Soc., 2011, 133, 18562-18565. The coordination of the chiral diol can dictate the chirality of the Ti centre $(\Delta, \Lambda)$ that transfers the chirality to the orientation of the $m$-aryl-diphenylphosphino groups $(\mathrm{Re} / \mathrm{Si})]$.
174.A. Duursma, R. Hoen, J. Schuppan, R. Hulst, A. J. Minnaard and B. L. Feringa, Org. Lett., 2003, 5, 3111-3113.

175.E. Hartmann and R. M. Gschwind, Angew. Chem. Int. Ed., 2013, 52, 2350-2354.

176.References 176-178 deal with metal complexes for which the respective position of the monophophine ligands (cis/trans) can be tuned through specific interaction with an additive; no catalytic applications. V. W.-W. Yam, X.-X. Lu and C.-C. Ko, Angew. Chem. Int. Ed., 2003, 42, 3385-3388.

177.X.-X. Lu, H.-S. Tang, C.-C. Ko, J. K.-Y. Wong, N. Zhu and V. W.-W. Yam, Chem. Commun., 2005, 1572-1574.

85 178.D. J. M. Snelders, M. A. Siegler, L. S. von Chrzanowski, A. L. Spek, G. van Koten and R. J. M. K. Gebbink, Dalton Trans., 2011, 40, 25882600.

179.D. J. M. Snelders, G. van Koten and R. J. M. K. Gebbink, Chem. Eur. J., 2011, 17, 42-57.

90 180.W. A. Herrmann, J. Kellner and H. Riepl, J. Organomet. Chem., 1990, 389, $103-128$.

181.R. Kreiter, R. J. M. K. Gebbink and G. van Koten, Tetrahedron, 2003, 59, 3989-3997.

182.D. J. M. Snelders, R. Kreiter, J. J. Firet, G. van Koten and R. J. M. K. Gebbink, Adv. Synth. Catal., 2008, 350, 262-266.

183.D. J. M. Snelders, G. van Koten and R. J. M. K. Gebbink, J. Am. Chem. Soc., 2009, 131, 11407-11416.

184.I. T. Horváth, R. V. Kastrup, A. A. Oswald and E. J. Mozeleski, Catal. Lett., 1989, 2, 85-90.

100 185.H. Ding and B. E. Hanson, J. Chem. Soc., Chem. Commun., 1994, 2747-2748.

186.H. Ding and B. E. Hanson, J. Mol. Cat. A: Chem., 1995, 99, 131-137.

187.H. Ding, B. E. Hanson and T. E. Glass, Inorg. Chim. Acta, 1995, 229, 329-333.

105 188.D. J. Darensbourg and C. J. Bischoff, Inorg. Chem., 1993, 32, 47-53.

189.H. Gulyás, J. Benet-Buchholz, E. C. Escudero-Adan, Z. Freixa and P. W. M. N. van Leeuwen, Chem. Eur. J., 2007, 13, 3424-3430.

190.L. Pignataro, B. Lynikaite, J. Cvengros, M. Marchini, U. Piarulli and C. Gennari, Eur. J. Org. Chem., 2009, 2539-2547.

110 191.G. Hattori, T. Hori, Y. Miyake and Y. Nishibayashi, J. Am. Chem. Soc., 2007, 129, 12930-12931.

192.Y. Li, Y. Feng, Y.-M. He, F. Chen, J. Pan and Q.-H. Fan, Tetrahedron Lett., 2008, 49, 2878-2881.

193.Y. Li, B. D. Ma, Y. M. He, F. Zhang and Q. H. Fan, Chem. Asian J., 2010, 5, 2454-2458.

194.For the influence of alkali cations on styrene hydroformylation reactions catalysed by rhodium complexes of bisphosphite ligands see references 194-196. S. B. Owens, Jr. and G. M. Gray, Organometallics, 2008, 27, 4282-4287.

120 195.A. A. Kaisare, S. B. Owens, Jr., E. J. Valente and G. M. Gray, J. Organomet. Chem., 2010, 695, 1472-1479.

196.A. A. Kaisare, S. B. Owens, Jr., E. J. Valente and G. M. Gray, J. Organomet. Chem., 2010, 695, 2658-2666.

197.I. Mon, D. A. Jose and A. Vidal-Ferran, Chem. Eur. J., 2013, 19, 2720 $125 \quad 2725$.

198.C. Machut, J. Patrigeon, S. Tilloy, H. Bricout, F. Hapiot and E. Monflier, Angew. Chem. Int. Ed., 2007, 46, 3040-3042.

199.J. Patrigeon, F. Hapiot, M. Canipelle, S. Menuel and E. Monflier, Organometallics, 2010, 29, 6668-6674.

130 200.For the various roles played by CDs in supramolecular catalysis see references 200 and 273: F. Hapiot, H. Bricout, S. Tilloy and E. Monflier, Eur. J. Inorg. Chem., 2012, 1571-1578.

201.B. Lynikaite, J. Cvengroš, U. Piarulli and C. Gennari, Tetrahedron Lett., 2008, 49, 755-759.

135 202.O. Chuzel, C. Magnier-Bouvier and E. Schulz, Tetrahedron: Asymmetry, 2008, 19, 1010-1019.

203.D. Didier and E. Schulz, ChemCatChem, 2011, 3, 1880-1884.

204.D. Didier, C. Magnier-Bouvier and E. Schulz, Adv. Synth. Catal., 2011, 353, 1087-1095.

140 205.S. Jónsson, F. G. J. Odille, P.-O. Norrby and K. Wärnmark, Chem. Commun., 2005, 549-551.

206.S. Jónsson, F. G. J. Odille, P.-O. Norrby and K. Wärnmark, Org. Biomol. Chem., 2006, 4, 1927-1948.

207.D.-Y. Ma, H. Norouzi-Arasi, E. Sheibani and K. Wärnmark, 145 Chem CatChem, 2010, 2, 629-632. 
208.E. Sheibani and K. Wärnmark, Org. Biomol. Chem., 2012, 10, 20592067.

209.J. Park, K. Lang, K. A. Abboud and S. Hong, J. Am. Chem. Soc., 2008, 130, 16484-16485.

5 210.J. Park, K. Lang, K. A. Abboud and S. Hong, Chem. Eur. J., 2011, 17, 2236-2245.

211.For light-driven organometallic catalysis by heterooligonuclear complexes see reviews 211 and 212: $\quad$ S. Rau, D. Walther and J. G. Vos, Dalton Trans., 2007, 915-919.

10 212.H. Ozawa and K. Sakai, Chem. Commun., 2011, 47, 2227-2242.

213. For examples of oligonuclear complexes prepared by a multi-step modification of the coordinated $\mathrm{N}$ or P ligands see references 213-218: M. Osawa, M. Hoshino and Y. Wakatsuki, Angew. Chem. Int. Ed., 2001, 40, 3472-3474.

15 214.J. Ekström, M. Abrahamson, C. Olson, J. Bergquist, F. B. Kaynak, L. Eriksson, L. Sun, H.-C. Becker, B. Åkermark, L. Hammarström and S. Ott, Dalton Trans., 2006, 4599-4606.

215.H. Ozawa, M. Haga and K. Sakai, J. Am. Chem. Soc., 2006, 128, 49264927.

20 216.H. Ozawa, Y. Yokoyama, M. Haga and K. Sakai, Dalton Trans., 2007, 1197-1206.

217.Y. Miyake, K. Nakajima, K. Sasaki, R. Saito, H. Nakanishi and Y. Nishibayashi, Organometallics, 2009, 28, 5240-5243.

218.F. Li, Y. Jiang, B. B. Zhang, F. Huang, Y. Gao and L. C. Sun, Angew. 25 Chem. Int. Ed., 2012, 51, 2417-2420.

219.For examples of oligonuclear complexes prepared by successive coordination of a metal catalyst and a sensitizer to a bridging ligand see references 219-226: S. Rau, B. Schaefer, D. Gleich, E. Anders, M. Rudolph, M. Friedrich, H. Goerls, W. Henry and J. G. Vos, Angew. Chem. Int. Ed., 2006, 45, 6215-6218.

220.M. Elvington, J. Brown, S. M. Arachchige and K. J. Brewer, J. Am. Chem. Soc., 2007, 129, 10644-10645.

221.A. Fihri, V. Artero, M. Razavet, C. Baffert, W. Leibl and M. Fontecave, Angew. Chem. Int. Ed., 2008, 47, 564-567.

35 222.W. M. Gao, J. H. Liu, W. N. Jiang, M. Wang, L. H. Weng, B. Åkermark and L. C. Sun, C. R. Chimie, 2008, 11, 915-921.

223.A. Fihri, V. Artero, A. Pereira and M. Fontecave, Dalton Trans., 2008 , 5567-5569.

224.W. Z. Chen, F. N. Rein and R. C. Rocha, Angew. Chem. Int. Ed., 2009, $40 \quad 48,9672-9675$.

225.W. Z. Chen, F. N. Rein, B. L. Scott and R. C. Rocha, Chem. Eur. J., 2011, 17, 5595-5604.

226.D. M. Cropek, A. Metz, A. M. Müller, H. B. Gray, T. Horne, D. C. Horton, O. Poluektov, D. M. Tiede, R. T. Weber, W. L. Jarrett, J. D. Phillips and A. A. Holder, Dalton Trans., 2012, 41, 13060-13073.

227.A. J. Esswein and D. G. Nocera, Chem. Rev., 2007, 107, 4022-4047.

228.K. Sakai and H. Ozawa, Coord. Chem. Rev., 2007, 251, 2753-2766.

229.M. Wang, Y. Na, M. Gorlov and L. Sun, Dalton Trans., 2009, 64586467.

50 230.A. M. Kluwer, R. Kapre, F. Hartl, M. Lutz, A. L. Spek, A. M. Brouwer, P. W. N. M. van Leeuwen and J. N. H. Reek, Proc. Natl. Acad. Sci. USA, 2009, 106, 10460-10465.

231.A related system where the zinc-porphyrin platform interacted with a pyridine group attached to the dithiolate ligand, was previously reported $\left(0.2 \mathrm{TON}\right.$ for the production of hydrogen from $\left.\mathrm{CF}_{3} \mathrm{COOH}\right)$ : X. Li, M. Wang, S. Zhang, J. Pan, Y. Na, J. Liu, B. Åkermark and L. Sun, J. Phys. Chem. B, 2008, 112, 8198-8202.

232.S. Jasimuddin, T. Yamada, K. Fukuju, J. Otsuki and K. Sakai, Chem. Commun., 2010, 46, 8466-8468.

60 233.N. Mourtzis, P. C. Carballada, M. Felici, R. J. M. Nolte, R. M. Willams, L. de Cola and M. C. Feiters, Phys. Chem. Chem. Phys., 2011, 13, 7903-7909.

234.P. C. Carballada, N. Mourtzis, M. Felici, S. Bonnet, R. J. M. Nolte, R. M. Williams, L. De Cola and M. C. Feiters, Eur. J. Org. Chem., 2012, 6729-6736.

235.M. Kirch, J. M. Lehn and J. P. Sauvage, Helv. Chim. Acta, 1979, 62, 1345-1384.

236.K. Kalyanasundaram, J. Kiwi and M. Grätzel, Hel. Chim. Acta, 1978, 61, 2720-2730.

70 237.J. Kiwi and M. Grätzel, Nature, 1979, 281, 657-658.

238.Y. Okuno, Y. Chiba and O. Yonemitsu, J. Chem. Soc., Chem. Commun., 1984, 1638-1639.

239.C. A. Tolman, Chem. Rev., 1977, 77, 313-348.
240.C. A. Tolman, J. Am. Chem. Soc., 1970, 92, 2953-2956.

75 241.J. Powell, J. Chem. Soc., Chem. Commun., 1989, 200-202.

242.W. P. Power and R. E. Wasylishen, Inorg. Chem., 1992, 31, 21762183.

243.E. Lindner, R. Fawzi, H. A. Mayer, K. Eichele and W. Hiller, Organometallics, 1992, 11, 1033-1043.

80 244.W. Leitner, M. Buhl, R. Fornika, C. Six, W. Baumann, E. Dinjus, M. Kessler, C. Kruger and A. Rufinska, Organometallics, 1999, 18, 11961206.

245.W. S. Knowles, Acc. Chem. Res., 1983, 16, 106-112.

246.M. T. Reetz and G. Mehler, Angew. Chem. Int. Ed., 2000, 39, 3889.

85 247.M. Van den Berg, A. J. Minnaard, E. P. Schudde, J. van Esch, A. H. M. De Vries and B. L. Feringa, J. Am. Chem. Soc., 2000, 122, 1153911540.

248.C. Claver, E. Fernandez, A. Gillon, K. Heslop, D. J. Hyett, A. Martorell, A. G. Orpen and P. G. Pringle, Chem. Commun., 2000, 961962.

249.M. T. Reetz, T. Sell, A. Meiswinkel and G. Mehler, Angew. Chem., Int. Ed., 2003, 42, 790-793.

250.M. T. Reetz, G. Mehler and A. Meiswinkel, Tetrahedron: Asymmetry, 2004, 15, 2165-2167.

95 251.M. T. Reetz and X. Li, Angew. Chem. Int. Ed., 2005, 44, 2959-2962.

252.G. J. H. Buisman, M. E. Martin, E. J. Vos, A. Klootwijk, P. C. J. Kamer and P. W. N. M. van Leeuwen, Tetrahedron: Asymmetry, 1995, 6, 719738.

253.M. Diéguez, A. Ruiz and C. Claver, J. Org. Chem., 2002, 67, 37963801.

254.S. R. Gilbertson, G. Chen and M. McLoughlin, J. Am. Chem. Soc., 1994, 116, 4481-4482.

255.M. B. Francis and E. N. Jacobsen, Angew. Chem. Int. Ed., 1999, 38, 937-941.

105 256.V. F. Slagt, P. C. J. Kamer, P. W. N. M. van Leeuwen and J. N. H. Reek, J. Am. Chem. Soc., 2004, 126, 1526-1536.

257.P. W. N. M. van Leeuwen and C. Claver, Rhodium-Catalyzed Hydroformylation, Kluwer Academic Publishers, Dordrecht, 2000.

258.M. Kuil, T. Soltner, P. W. N. M. van Leeuwen and J. N. H. Reek, $J$. Am. Chem. Soc., 2006, 128, 11344-11345.

259.T. Besset, D. W. Norman and J. N. H. Reek, Adv. Synth. Catal., 2013, 355, 348-352.

260.A. W. Kleij, M. Lutz, A. L. Spek, P. W. N. M. van Leeuwen and J. N. H. Reek, Chem. Commun., 2005, 3661-3663.

115 261.A. W. Kleij, M. Kuil, D. M. Tooke, A. L. Spek and J. N. H. Reek, Inorg. Chem., 2005, 44, 7696-7698.

262.V. Bocokić, M. Lutz, A. L. Spek and J. N. H. Reek, Dalton Trans., 2012, 41, 3740-3750.

263.R. Bellini, S. H. Chikkali, G. Berthon-Gelloz and J. N. H. Reek, 120 Angew. Chem. Int. Ed., 2011, 50, 7342-7345.

264.R. Bellini and J. N. H. Reek, Chem. Eur. J., 2012, 18, 7091-7099.

265.M. Rubio, E. Jellema, M. A. Siegler, A. L. Spek, J. N. H. Reek and B. de Bruin, Dalton Trans., 2009, 8970-8976.

266.M. Rubio, M. A. Siegler, A. L. Spek and J. N. H. Reek, Dalton Trans., 2010, 39, 5432-5435.

267.G. A. Morris, S. T. Nguyen and J. T. Hupp, J. Mol. Cat. A: Chem., 2001, 174, 15-20.

268.J. Flapper and J. N. H. Reek, Angew. Chem. Int. Ed., 2007, 46, 85908592.

130 269.J. A. Fuentes, M. L. Clarke and A. M. Z. Slawin, New. J. Chem., 2008, 32, 689-693.

270.K. Ohmatsu, M. Ito, T. Kunieda and T. Ooi, Nature Chem., 2012, 4, 473-477.

271.K. Ohmatsu, M. Ito, T. Kunieda and T. Ooi, J. Am. Chem. Soc., 2013, 135, 590-593.

272.M. Rueping, R. M. Koenigs and I. Atodiresei, Chem. Eur. J., 2010, 16, 9350-9365.

273.F. Hapiot, S. Tilloy and E. Monflier, Chem. Rev., 2006, 106, 767-781.

274.J. Bjerre, C. Rousseau, L. Marinescu and M. Bols, Appl. Microbiol. 140 Biotechnol., 2008, 81, 1-11.

275.L. Caron, M. Canipelle, S. Tilloy, H. Bricout and E. Monflier, Eur. $J$ Inorg.Chem., 2003, 595-599.

276.E. Monflier, H. Bricout, F. Hapiot, S. Tilloy, A. Aghmiz and MasdeuBultó, Adv. Synth. Catal., 2004, 346, 425-431.

145 277.C. Binkowski-Machut, M. Canipelle, H. Bricout, S. Tilloy, F. Hapiot and E. Monflier, Eur. J. Inorg.Chem., 2006, 1611-1919. 
278.E. Monflier, S. Tilloy, G. Fremy, Y. Castanet and A. Mortreux, Tetrahedron Lett., 1995, 36, 9481-9484.

279.L. Caron, H. Bricout, S. Tilloy, A. Ponchel, D. Landy, S. Fourmentin and E. Monflier, Adv. Synth. Catal., 2004, 346, 1449-1456.

5 280.L. Leclercq, F. Hapiot, S. Tilloy, K. Ramkisoensing, J. N. H. Reek, P. W. N. M. van Leeuwen and E. Monflier, Organometallics, 2005, 24, 2070-2075.

281.L. Leclercq and A. R. Schmitzer, Organometallics, 2010, 29, 34423449 .

10 282.D. N. Tran, F. X. Legrand, S. Menuel, H. Bricout, S. Tilloy and E. Monflier, Chem. Commun., 2012, 48, 753-755.

283.R. Bellini and J. N. H. Reek, Chem. Eur. J., 2012, 18, 13510-13519.

284.P. Dydio, C. Rubay, T. Gadzikwa, M. Lutz and J. N. H. Reek, J. Am. Chem. Soc., 2011, 133, 17176-17179.

15285 . For the anion binding ability of the 7,7'-diamido-2,2'diindolylmethane moiety see references 285-286: P. Dydio, T. Zieliński and J. Jurczak, Chem. Commun., 2009, 4560-4562.

286.P. Dydio, T. Zieliński and J. Jurczak, Org. Lett., 2010, 12, 1076-1078.

287.H. C. Kolb, P. G. Andersson and K. B. Sharpless, J. Am. Chem. Soc.,

$20 \quad 1994,116,1278-1291$.

288.K. B. Sharpless, Tetrahedron, 1994, 50, 4235-4258.

289.E. J. Corey and M. C. Noe, J. Am. Chem. Soc., 1996, 118, $11038-$ 11053.

290.D. W. Nelson, A. Gypser, P. T. Ho, H. C. Kolb, T. Kondo, H. L.

25 Kwong, D. V. McGrath, A. E. Rubin, P. O. Norrby, K. P. Gable and K. B. Sharpless, J. Am. Chem. Soc., 1997, 119, 1840-1858.

291.A. J. DelMonte, J. Haller, K. N. Houk, K. B. Sharpless, D. A. Singleton, T. Strassner and A. A. Thomas, J. Am. Chem. Soc., 1997, 119, $9907-9908$.

30 292.E. J. Corey, A. Guzman-Perez and M. C. Noe, J. Am. Chem. Soc., 1994, 116, 12109-12110.

293.E. J. Corey, M. C. Noe and S. Sarshar, Tetrahedron Lett., 1994, 35, 2861-2864.

294.E. J. Corey, A. Guzmanperez and M. C. Noe, Tetrahedron Lett., 1995, 35 36, 3481-3484.

295.D. A. Evans, K. T. Chapman and J. Bisaha, J. Am. Chem. Soc., 1988, 110, $1238-1256$.

296.E. J. Corey and T.-P. Loh, J. Am. Chem. Soc., 1991, 113, 8966-8967.

297.E. J. Corey, T. P. Loh, T. D. Roper, M. D. Azimioara and M. C. Noe, J. Am. Chem. Soc., 1992, 114, 8290-8292.

298.Y. Hayashi, J. J. Rohde and E. J. Corey, J. Am. Chem. Soc., 1996, 118, 5502-5503.

299.E. J. Corey and J. J. Rohde, Tetrahedron Lett., 1997, 38, 37-40.

300.F. Doro, J. N. H. Reek and P. W. N. M. van Leeuwen,

45 Organometallics, 2010, 29, 4440-4447.

301.L. A. Castonguay, A. K. Rappé and C. J. Casewit, J. Am. Chem. Soc., 1991, 113, 7177-7183.

302.F. Li, Z. J. Zheng, J. Y. Shang, K. Z. Jiang, G. Q. Lai, J. X. Jiang and L. W. Xu, Chem. Asian J., 2012, 7, 2008-2013.

50 303.H. Nakajima, M. Yasuda, R. Takeda and A. Baba, Angew. Chem. Int. Ed., 2012, 51, 3867-3870.

304.S. France, M. H. Shah, A. Weatherwax, H. Wack, J. P. Roth and T. Lectka, J. Am. Chem. Soc., 2005, 127, 1206-1215.

305. For reviews dealing with cooperative homo- and heteromultimetallic

55 catalysts see references 305-315: M. Shibasaki and H. Sasai, Pure Appl. Chem., 1996, 68, 523-530.

306.M. Shibasaki, H. Sasai and T. Arai, Angew. Chem. Int. Ed. Engl., 1997, 36, $1236-1256$.

307.M. Shibasaki, M. Kanai and K. Funabashi, Chem. Commun., 2002, 1989-1999.

308.M. Shibasaki and N. Yoshikawa, Chem. Rev., 2002, 102, 2187-2209.

309.M. Shibasaki, M. Kanai, S. Matsunaga and N. Kumagai, Acc. Chem. Res., 2009, 42, 1117-1127.

310.R. G. Konsler, J. Karl and E. N. Jacobsen, J. Am. Chem. Soc., 1998,

65 120, 10780-10781.

311.H. Steinhagen and G. Helmchen, Angew. Chem. Int. Ed. Engl., 1996, 35, 2339-2342.

312.G. J. Rowlands, Tetrahedron, 2001, 57, 1865-1882.

313.J.-A. Ma and D. Cahard, Angew. Chem. Int. Ed., 2004, 43, 4566-4583.

70 314.D. H. Paull, C. J. Abraham, M. T. Scerba, E. Alden-Danforth and T. Lectka, Acc. Chem. Res., 2008, 41, 655-663.

315.R. M. Haak, S. J. Wezenberg and A. W. Kleij, Chem. Commun., 2010, 46, 2713-2723.
316.H. Gröger, Chem. Eur. J., 2001, 7, 5247-5251.

75 317.Y.-M. Lin, J. Boucau, Z. Li, V. Casarotto, J. Lin, A. N. Nguyen and J. Ehrmantraut, Org. Lett., 2007, 9, 567-570.

318.For a review on bifunctional catalysts that combine a metal centre and a cinchona alkaloid scaffold see: L. Stegbauer, F. Sladojevich and D. J. Dixon, Chem. Sci., 2012, 3, 942-958.

80 319.A. Borner, J. Ward, K. Kortus and H. B. Kagan, Tetrahedron: Asymmetry, 1993, 4, 2219-2228.

320.L. B. Fields and E. N. Jacobsen, Tetrahedron: Asymmetry, 1993, 4, 2229-2240.

321.For avant la lettre examples of bifunctional metal catalysts see the

85 following review and references therein: M. Sawamura and Y. Ito, Chem. Rev., 1992, 92, 857-871.

322.Y. Ito, M. Sawamura and T. Hayashi, J. Am. Chem. Soc., 1986, 108, 6405-6406.

323.Y. Ito, M. Sawamura and T. Hayashi, Tetrahedron Lett., 1987, 28, $90 \quad 6215-6218$.

324.M. Sawamura, Y. Ito and T. Hayashi, Tetrahedron Lett., 1990, 31, 2723-2726.

325.T. Hayashi, M. Sawamura and Y. Ito, Tetrahedron, 1992, 48, 19992012.

95 326.T. Hayashi, M. Kawano and Y. Ito, J. Am. Chem. Soc., 1987, 109, 7876-7878.

327.M. Yatagai, M. Zama, T. Yamagishi and M. Hida, Chem. Lett., 1983, 1203-1206.

328.M. Yatagai, M. Zama, T. Yamagishi and M. Hida, Bull. Chem. Soc. Jpn., 1984, 57, 739-746.

329.T. Yamagishi, S. Ikeda, T. Egawa, M. Yamaguchi and M. Hida, Bull. Chem. Soc. Jpn., 1990, 63, 281-283.

330.M. Yamakawa, H. Ito and R. Noyori, J. Am. Chem. Soc., 2000, 122, 1466-1478.

105 331.R. Noyori, M. Yamakawa and S. Hashiguchi, J. Org. Chem., 2001, 66, 7931-7944.

332.M. Yamakawa, I. Yamada and R. Noyori, Angew. Chem. Int. Ed., 2001, 40, 2818-2821.

333.R. H. Morris, Chem. Soc. Rev., 2009, 38, 2282-2291.

110 334.S. E. Clapham and R. H. Morris, Organometallics, 2005, 24, 479-481.

335.W. Baratta, M. Ballico, G. Chelucci, K. Siega and P. Rigo, Angew. Chem. Int. Ed., 2008, 47, 4362-4365.

336.W. Baratta, C. Barbato, S. Magnolia, K. Siega and P. Rigo, Chem. Eur. $J ., 2010,16,3201-3206$.

115 337.W. Baratta, L. Fanfoni, S. Magnolia, K. Siega and P. Rigo, Eur. J. Inorg.Chem., 2010, 1419-1423.

338.M. Bertoli, A. Choualeb, D. G. Gusev, A. J. Lough, Q. Major and B. Moore, Dalton Trans., 2011, 40, 8941-8949.

339.M. Bertoli, A. Choualeb, A. J. Lough, B. Moore, D. Spasyuk and D.

120 G. Gusev, Organometallics, 2011, 30, 3479-3482.

340.D. Spasyuk, S. Smith and D. G. Gusev, Angew. Chem. Int. Ed., 2012, 51, 2772-2775.

341.T. Ikariya and A. J. Blacker, Acc. Chem. Res., 2007, 40, 1300-1308.

342.M. Ito and T. Ikariya, Chem. Commun., 2007, 5134-5142.

125 343.T. Ikariya and I. D. Gridnev, Chem. Rec., 2009, 9, 106-123.

344.T. Ikariya and I. D. Gridnev, Top. Catal., 2010, 53, 894-901.

345.For reactivity of protic-NHC complexes see the following reference and reference 379: V. Miranda-Soto, D. B. Grotjahn, A. L. Cooksy, J. A. Golen, C. E. Moore and A. L. Rheingold, Angew. Chem. Int. Ed., 2011, 50, 631-635.

346.K. Abdur-Rashid, S. E. Clapham, A. Hadzovic, J. N. Harvey, A. J. Lough and R. H. Morris, J. Am. Chem. Soc., 2002, 124, 15104-15118.

347.C. A. Sandoval, T. Ohkuma, N. Utsumi, K. Tsutsumi, K. Murata and R. Noyori, Chem. Asian J., 2006, 1, 102-110.

135 348.J. S. M. Samec, J. E. Backvall, P. G. Andersson and P. Brandt, Chem. Soc. Rev., 2006, 35, 237-248.

349.D. Di Tommaso, S. A. French and C. R. A. Catlow, THEOCHEM, 2007, 812, 39-49.

350.D. Di Tommaso, S. A. French, A. Zanotti-Gerosa, F. Hancock, E. J. 140 Palin and C. R. A. Catlow, Inorg. Chem., 2008, 47, 2674-2687.

351.H. Y. T. Chen, D. Di Tommaso, G. Hogarth and C. R. A. Catlow, Dalton Trans., 2011, 40, 402-412.

352.A different mechanism was proposed by Fan and co-workers for the hydrogenation of quinoline derivatives with $\left[\operatorname{Ru}(\mathrm{TsDPEN})\left(\eta^{6}\right.\right.$-arene $\left.)\right]$ complexes. In this case, a transition state is proposed in which the cationic $\mathrm{Ru}$ catalyst, the anion and the substrate interact through 
several secondary interactions (see Fig. 61)].: T. Wang, L.-G. Zhuo, Z. Li, F. Chen, Z. Ding, Y. He, Q.-H. Fan, J. Xiang, Z.-X. Yu and A. S. C. Chan, J. Am. Chem. Soc., 2011, 133, 9878-9891.

353.M. Watanabe, K. Murata and T. Ikariya, J. Am. Chem. Soc., 2003, 125, 7508-7509.

354.M. Watanabe, A. Ikagawa, H. Wang, K. Murata and T. Ikariya, J. Am. Chem. Soc., 2004, 126, 11148-11149.

355.T. Ikariya, H. Wang, M. Watanabe and K. Murata, J. Organomet. Chem., 2004, 689, 1377-1381.

10 356.R. Guo, R. H. Morris and D. Song, J. Am. Chem. Soc., 2005, 127, $516-$ 517.

357.R. Guo, X. Chen, C. Elpelt, D. Song and R. H. Morris, Org. Lett., 2005, 7, $1757-1759$.

358.H. Wang, M. Watanabe and T. Ikariya, Tetrahedron Lett., 2005, 46, $15 \quad 963-966$.

359.S. E. Clapham, R. Guo, M. Z.-D. Iuliis, N. Rasool, A. Lough and R. H. Morris, Organometallics, 2006, 25, 5477-5486.

360.Y. Hasegawa, M. Watanabe, I. D. Gridnev and T. Ikariya, J. Am. Chem. Soc., 2008, 130, 2158-2159.

20 361.Y. Hasegawa, I. D. Gridnev and T. Ikariya, Angew. Chem. Int. Ed., 2010, 49, 8157-8160.

362.I. D. Gridnev, M. Watanabe, H. Wang and T. Ikariya, J. Am. Chem. Soc., 2010, 132, 16637-16650.

363.For the use of chiral TF-BiphamPhos ligands in catalysis see the

25 following reference and references therein: M. Wang, C.-J. Wang and Z. Lin, Organometallics, 2012, 31, 7870-7876.

364.Z.-Y. Xue, Q.-H. Li, H.-Y. Tao and C.-J. Wang, J. Am. Chem. Soc., 2011, 133, 11757-111765.

365.D. B. Grotjahn, C. D. Incarvito and A. L. Rheingold, Angew. Chem. $30 \quad$ Int. Ed., 2001, 40, 3884-3887.

366.F. A. Jalon, B. R. Manzano, A. Caballero, M. C. Carrion, L. Santos, G. Espino and M. Moreno, J. Am. Chem. Soc., 2005, 127, 15364-15365.

367.D. B. Grotjahn and D. A. Lev, J. Am. Chem. Soc., 2004, 126, 1223212233.

35 368.D. B. Grotjahn, Top. Catal., 2010, 53, 1009-1014.

369.T. Suzuki, M. Tokunaga and Y. Wakatsuki, Org. Lett., 2001, 3, 735 737.

370.D. B. Grotjahn, Chem. Eur. J., 2005, 11, 7146-7153.

371.D. B. Grotjahn, Dalton Trans., 2008, 6497-6508.

40 372.D. B. Grotjahn, Chem. Lett., 2010, 39, 908-914.

373.T. Oshiki, H. Yamashita, K. Sawada, M. Utsunomiya, K. Takahashi and K. Takai, Organometallics, 2005, 24, 6287-6290.

374.W. K. Fung, X. Huang, M. L. Man, S. M. Ng, M. Y. Hung, Z. Y. Lin and C. P. Lau, J. Am. Chem. Soc., 2003, 125, 11539-11544.

45 375.P. Daw, A. Sinha, S. M. W. Rahaman, S. Dinda and J. K. Bera, Organometallics, 2012, 31, 3790-3797.

376.D. B. Grotjahn, C. R. Larsen, J. L. Gustafson, R. Nair and A. Sharma, J. Am. Chem. Soc., 2007, 129, 9592-9593.

377.G. Erdogan and D. B. Grotjahn, J. Am. Chem. Soc., 2009, 131, 10354$50 \quad 10355$.

378.D. B. Grotjahn, J. E. Kraus, H. Amouri, M. N. Rager, A. L. Cooksy, A. J. Arita, S. A. Cortes-Llamas, A. A. Mallari, A. G. DiPasquale, C. E. Moore, L. M. Liable-Sands, J. D. Golen, L. N. Zakharov and A. L. Rheingold, J. Am. Chem. Soc., 2010, 132, 7919-7934.

55 379.N. Meier, F. E. Hahn, T. Pape, C. Siering and S. R. Waldvogel, Eur. J. Inorg.Chem., 2007, 1210-1214.

380.For a review on pyrazole and protic-NHC complexes see: S. Kuwata and T. Ikariya, Chem. Eur. J., 2011, 17, 3542-3556.

381. L. Bellarosa, J. Díez, J. Gimeno, A. Lledós, F. J. Suárez, G. Ujaque

60 and C. Vicent, Chem. Eur. J., 2012, 18, 7749-7765.

382.S. Otto and J. B. F. N. Engberts, Pure Appl. Chem., 2000, 72, 1365 1372.

383.Y. S. Jung and R. A. Marcus, J. Am. Chem. Soc., 2007, 129, $5492-$ 5502.

65 384.X. Wu, J. Liu, D. Di Tommaso, J. A. Iggo, C. R. A. Catlow, J. Bacsa and J. Xiao, Chem. Eur. J., 2008, 14, 7699-7715.

385.M. A. Iron, E. Ben-Ari, R. Cohen and D. Milstein, Dalton Trans., 2009, 9433-9439.

386.G. Kovacs, A. Lledós and G. Ujaque, Organometallics, 2010, 29, 3252-3260.

387.M. R. DuBois and D. L. DuBois, Chem. Soc. Rev., 2009, 38, 62-72.

388.M. R. Dubois and D. L. Dubois, Acc. Chem. Res., 2009, 42, 1974-1982.
389.A. D. Wilson, R. K. Shoemaker, A. Miedaner, J. T. Muckerman, D. L. DuBois and M. R. DuBois, Proc. Natl. Acad. Sci. U. S. A., 2007, 104, 6951-6956.

390.A. D. Wilson, R. H. Newell, M. J. McNevin, J. T. Muckerman, M. R. DuBois and D. L. DuBois, J. Am. Chem. Soc., 2006, 128, 358-366.

391.J. Y. Yang, R. M. Bullock, W. J. Shaw, B. Twamley, K. Fraze, M. R. DuBois and D. L. DuBois, J. Am. Chem. Soc., 2009, 131, 5935-5945.

80 392.G. M. Jacobsen, J. Y. Yang, B. Twamley, A. D. Wilson, R. M. Bullock, M. R. DuBois and D. L. DuBois, Energy Environ. Sci., 2008, 1, 167174.

393.D. R. Jensen, M. J. Schultz, J. A. Mueller and M. S. Sigman, Angew. Chem. Int. Ed., 2003, 42, 3810-3813.

85 394.J. A. Mueller, C. P. Goller and M. S. Sigman, J. Am. Chem. Soc., 2004, 126, 9724-9734.

395.B. A. Persson, A. L. E. Larsson, M. Le Ray and J. E. Backvall, J. Am. Chem. Soc., 1999, 121, 1645-1650.

396.B. L. Conley, M. K. Pennington-Boggio, E. Boz and T. J. Williams, Chem. Rev., 2010, 110, 2294-2312.

397.C. P. Casey, S. W. Singer, D. R. Powell, R. K. Hayashi and M. Kavana, J. Am. Chem. Soc., 2001, 123, 1090-1100.

398.C. P. Casey, G. A. Bikzhanova, Q. Cui and I. A. Guzei, J. Am. Chem. Soc., 2005, 127, 14062-14071.

95 399.C. P. Casey, S. E. Beetner and J. B. Johnson, J. Am. Chem. Soc., 2008, 130, 2285-2295.

400.A. Comas-Vives, G. Ujaque and A. Lledós, Organometallics, 2008, 27, 4854-4863.

401.H. J. Knolker, E. Baum, H. Goesmann and R. Klauss, Angew. Chem. $100 \quad$ Int. Ed., 1999, 38, 2064-2066.

402.C. P. Casey and H. R. Guan, J. Am. Chem. Soc., 2007, 129, 5816-5817.

403.H. H. Zhang, D. Z. Chen, Y. H. Zhang, G. Q. Zhang and J. B. Liu, Dalton Trans., 2010, 39, 1972-1978.

404.T. Šmejkal and B. Breit, Angew. Chem. Int. Ed., 2008, 47, 311-315.

105 405.T. Šmejkal, D. Gribkov, J. Geier, M. Keller and B. Breit, Chem. Eur. J., 2010, 16, 2470-2478.

406.T. Šmejkal and B. Breit, Angew. Chem. Int. Ed., 2008, 47, 3946-3949.

407.L. Diab, T. Šmejkal, J. Geier and B. Breit, Angew. Chem. Int. Ed., 2009, 48, 8022-8026.

110 408.T. Hayashi, K. Kanehira, H. Tsuchiya and M. Kumada, J. Chem. Soc., Chem. Commun., 1982, 1162-1164.

409.M. Sawamura, H. Nagata, H. Sakamoto and Y. Ito, J. Am. Chem. Soc., 1992, 114, 2586-2592.

410.M. Sawamura, Y. Nakayama, W.-M. Tang and Y. Ito, J. Org. Chem., 1996, 61, 9090-9096.

411.T. Hayashi, A. Yamamoto, T. Hagihara and Y. Ito, Tetrahedron Lett., 1986, 27, 191-194.

412.T. Hayashi, K. Kanehira, T. Hagihara and M. Kumada, J. Org. Chem., 1988, 53, 113-120.

120 413.T. Hayashi, A. Yamamoto, Y. Ito, E. Nishioka, H. Miura and K. Yanagi, J. Am. Chem. Soc., 1989, 111, 6301-6311.

414.T. Hayashi, K. Kishi, A. Yamamoto and Y. Ito, Tetrahedron Lett., 1990, 31, 1743-1746.

415.B. M. Trost, M. R. Machacek and A. Aponick, Acc. Chem. Res., 2006, 39, 747-760.

416.W. Zeng, G.-Y. Chen, Y.-G. Zhou and Y.-X. Li, J. Am. Chem. Soc., 2007, 129, 750-751.

417.A. Börner, Eur. J. Inorg.Chem., 2001, 327-337 and references therein.

418.T. Hayashi, T. Mise and M. Kumada, Tetrahedron Lett., 1976, 48, $130 \quad 4351-4354$.

419.T. Hayashi, K. Kanehira and M. Kumada, Tetrahedron Lett., 1981, 22, 4417-4420.

420.O. Riant, O. Samuel, T. Flessner, S. Taudien and H. B. Kagan, J. Org. Chem., 1997, 62, 6733-6745.

135 421.D. Carmichael, H. Doucet and J. M. Brown, Chem. Commun., 1999, 261-262.

422.M. Livieri, F. Mancin, U. Tonellato and J. Chin, Chem. Commun., 2004, 2862-2863.

423.G. Feng, J. C. Mareque-Rivas, R. T. M. de Rosales and N. H. Williams, J. Am. Chem. Soc., 2005, 127, 13470-13471.

424.G. Q. Feng, J. C. Mareque-Rivas and N. H. Williams, Chem. Commun., 2006, 1845-1847.

425.G. Feng, D. Natale, R. Prabaharan, J. C. Mareque-Rivas and N. H. Williams, Angew. Chem. Int. Ed., 2006, 45, 7056-7059. 
426.M. Livieri, F. Mancin, G. Saielli, J. Chin and U. Tonellato, Chem. Eur. $J ., 2007,13,2246-2256$.

427.M. Wall, B. Linkletter, D. Williams, A.-M. Lebuis, R. C. Hynes and J. Chin, J. Am. Chem. Soc., 1999, 121, 4710-4711.

5 428.J. Chin, S. Chung and D. H. Kim, J. Am. Chem. Soc., 2002, 124, 10948. 429.D. Natale and J. C. Mareque-Rivas, Chem. Commun., 2008, 425-437.

430.R. Bonomi, F. Selvestrel, V. Lombardo, C. Sissi, S. Polizzi, F. Mancin, U. Tonellato and P. Scrimin, J. Am. Chem. Soc., 2008, 130, 1574415745.

10 431.E. Kövari and R. Kramer, J. Am. Chem. Soc., 1996, 118, 12704-12709.

432.Y. An, M.-L. Tong, L.-N. Ji and Z.-W. Mao, Dalton Trans., 2006, 2066-2071.

433.J.-H. Li, J.-T. Wang, Z.-W. Mao and L.-N. Ji, Inorg. Chem. Commun., 2008, 11, 865-868.

15 434.J.-H. Li, J.-T. Wang, P. Hu, L.-Y. Zhang, Z.-N. Chen, Z.-W. Mao and L.-N. Ji, Polyhedron, 2008, 27, 1898-1904.

435.J.-H. Li, J.-T. Wang, L.-Y. Zhang, Z.-N. Chen, Z.-W. Mao and L.-N. Ji, Inorg. Chim. Acta, 2009, 362, 1918-1924.

436.H. Ait-Haddou, J. Sumaoka, S. L. Wiskur, J. F. Folmer-Andersen and

20 E. V. Anslyn, Angew. Chem. Int. Ed., 2002, 41, 4014-4016.

437.J. He, J. Sun, Z.-W. Mao, L.-N. Ji and H. Sun, J. Inorg. Biochem., 2009, 103, 851-858.

438.J. He, P. Hu, Y.-J. Wang, M.-L. Tong, H. Sun, Z.-W. Mao and L.-N. Ji, Dalton Trans., 2008, 3207-3214.

25 439.R. Bonomi, G. Saielli, U. Tonellato, P. Scrimin and F. Mancin, J. Am. Chem. Soc., 2009, 131, 11278-11279.

440.T. Kull, J. Cabrera and R. Peters, Chem. Eur. J., 2010, 16, 9132-9139.

441.S. Das, C. D. Incarvito, R. H. Crabtree and G. W. Brudvig, Science, 2006, 312, 1941-1943.

30 442.S. Das, G. W. Brudvig and R. H. Crabtree, Chem. Commun., 2008, 413-424.

443.S. Das, G. W. Brudvig and R. H. Crabtree, J. Am. Chem. Soc., 2008, 130, 1628-1637.

444.D. Balcells, P. Moles, J. D. Blakemore, C. Raynaud, G. W. Brudvig,

${ }_{35}$ R. H. Crabtree and O. Eisenstein, Dalton Trans., 2009, 5989-6000.

445.J. F. Hull, E. L. O. Sauer, C. D. Incarvito, J. W. Faller, G. W. Brudvig and R. H. Crabtree, Inorg. Chem., 2009, 48, 488-495.

446.P. A. Ulmann, A. B. Braunschweig, O.-S. Lee, M. J. Wiester, G. C. Schatz and C. A. Mirkin, Chem. Commun., 2009, 5121-5123.

40 447.F. Voss, E. Herdtweck and T. Bach, Chem. Commun., 2011, 47, $2137-$ 2139.

448.P. Fackler, C. Berthold, F. Voss and T. Bach, J. Am. Chem. Soc., 2010, 132, 15911-15913.

449.P. Fackler, S. M. Huber and T. Bach, J. Am. Chem. Soc., 2012, 134, $45 \quad 12869-12878$.

450.Y. Chen, K. B. Fields and X. P. Zhang, J. Am. Chem. Soc., 2004, 126, 14718-14719.

451.Y. Chen and X. P. Zhang, J. Org. Chem., 2007, 72, 5931-5934.

452.Y. Chen, J. V. Ruppel and X. P. Zhang, J. Am. Chem. Soc., 2007, 129, 12074-12075.

453.S. F. Zhu, J. A. Perman and X. P. Zhang, Angew. Chem. Int. Ed., 2008, 47, 8460-8463.

454.S. Zhu, J. V. Ruppel, H. Lu, L. Wojtas and X. P. Zhang, J. Am. Chem. Soc., 2008, 130, 5042-5043.

55 455.W. I. Dzik, X. Xu, X. P. Zhang, J. N. H. Reek and B. de Bruin, J. Am. Chem. Soc., 2010, 132, 10891-10902.

456.P. Dydio, W. I. Dzik, M. Lutz, B. de Bruin and J. N. H. Reek, Angew. Chem. Int. Ed., 2011, 50, 396-400.

457.P. Dydio and J. N. H. Reek, Angew. Chem. Int. Ed., 2013, 52, 38783882 .

458.For selected examples of supramolecular catalysis in the solid state see references 458-461: M. Pattabiraman, A. Natarajan, L. Kaanumalle and V. Ramamurthy, Org. Lett., 2005, 7, 529-532.

459.A. N. Sokolov, D.-K. Bučar, J. Baltrusaitis, S. X. Gu and L. R.

65 MacGillivray, Angew. Chem. Int. Ed., 2010, 49, 4273-4277.

460.B. R. Bhogala, B. Captain, A. Parthasarathy and V. Ramamurthy, $J$. Am. Chem. Soc., 2010, 132, 13434-13442.

461.E. Elacqua, P. Kaushik, R. H. Groeneman, J. C. Sumrak, D. K. Bucar and L. R. MacGillivray, Angew. Chem. Int. Ed., 2012, 51, 1037-1041.

70 462.B. List, R. A. Lerner and C. F. Barbas III, J. Am. Chem. Soc., 2000, 122, 2395-2396.

463.K. A. Ahrendt, C. J. Borths and D. W. C. MacMillan, J. Am. Chem. Soc., 2000, 122, 4243-4244.
464.For general reviews on organocatalysis including aminocatalysis see 75 references 464-468: P. I. Dalko and L. Moisan, Angew. Chem. Int. Ed., 2004, 43, 5138-5175.

465.M. J. Gaunt, C. C. C. Johansson, A. McNally and N. T. Vo, Drug Discovery Today, 2007, 12, 8-27.

466.A. Dondoni and A. Massi, Angew. Chem. Int. Ed., 2008, 47, 4638$80 \quad 4660$.

467.P. Melchiorre, M. Marigo, A. Carlone and G. Bartoli, Angew. Chem. Int. Ed., 2008, 47, 6138-6171.

468.A. Erkkilä, I. Majander and P. M. Pihko, Chem. Rev., 2007, 107, 54165470 and references therein.

85 469.L. Zhang and S. Luo, Synlett, 2012, 23, 1575-1589.

470.D. B. Ramachary, R. Sakthidevi and K. S. Shruthi, Chem. Eur. J., 2012, 18, 8008-8012.

471.For general reviews on catalytic systems that combine a metal catalyst and an organocatalyst see references 52, 53, 272 and 471-475: Z. Shao and H. Zhang, Chem. Soc. Rev., 2009, 38, 2745-2755.

472.C. Zhong and X. Shi, Eur. J. Org. Chem., 2010, 2999-3025.

473.Z. Yu, W. Jin and Q. Jiang, Angew. Chem. Int. Ed., 2012, 51, 60606072.

474.A. E. Allen and D. W. C. MacMillan, Chem. Sci., 2012, 3, 633-658.

95 475.Z. Du and Z. Shao, Chem. Soc. Rev., 2013, 42, 1337-1378.

476.H. Xu, S. J. Zuend, M. G. Woll, Y. Tao and E. N. Jacobsen, Science, 2010, 327, 986-990.

477.A. Sakakura, M. Sakuma and K. Ishihara, Org. Lett., 2011, 13, 31303133 .

100 478.S. E. Denmark and M. T. Burk, Org. Lett., 2012, 14, 256-259.

479.D. Leow and C.-H. Tan, Synlett, 2010, 11, 1589-1605.

480.Y. Takemoto, Chem. Pharm. Bull., 2010, 58, 593-601.

481.Z. Zhang and P. R. Schreiner, Chem. Soc. Rev., 2009, 38, 1187-1198.

482.Y. Takemoto, Org. Biomol.Chem., 2005, 3, 4299-4306.

105 483.M. Terada, Synthesis, 2010, 1929-1982.

484. Chiral phosphate anion can also act as a bridge between imine substrates and the Hantzsch ester: L. Simon and J. M. Goodman, J. Am. Chem. Soc., 2008, 130, 8741-8747.

485.For papers dealing with the controversial mode of action of chiral 110 phosphoric acids see references 485-487: M. Hatano, K. Moriyama, T. Maki and K. Ishihara, Angew. Chem. Int. Ed., 2010, 49, 3823-3826.

486.F. Drouet, C. Lalli, H. Liu, G. Masson and J. Zhu, Org. Lett., 2011, 13, 94-97.

487.M. Fleischmann, D. Drettwan, E. Sugiono, M. Rueping and R. M. 115 Gschwind, Angew. Chem. Int. Ed., 2011, 50, 6364-6369.

488.S. J. Connon, Chem. Commun., 2008, 2499-2510.

489.T. Marcelli and H. Hiemstra, Synthesis, 2010, 8, 1229-1279.

490.K. Ohmatsu, M. Kiyokawa and T. Ooi, J. Am. Chem. Soc., 2011, 133, 1307-1309.

120 491.For early examples of achiral templates, see references 491-494: J. Wolfe, D. Nemeth, A. Costero and J. Rebek, Jr., J. Am. Chem. Soc., 1988, 110, 983-984.

492.T. R. Kelly, C. Zhao and G. J. Bridger, J. Am. Chem. Soc., 1989, 111, 3744-3745.

125 493.T. R. Kelly, G. J. Bridger and C. Zhao, J. Am. Chem. Soc., 1990, 112, 8024-8034.

494.P. Tecilla, S.-K. Chang and A. D. Hamilton, J. Am. Chem. Soc., 1990, 112, 9586-9590.

495.D. M. Bassani, V. Darcos, S. Mahony and J.-P. Desvergne, J. Am. Chem. Soc., 2000, 122, 8795-8796.

496.N. McCleanaghan, C. Absalon and D. M. Bassani, J. Am. Chem. Soc., 2003, 125, 13004-13005.

497.B. Grosch, C. N. Orlebar, E. Herdtweck, W. Massa and T. Bach, Angew. Chem. Int. Ed., 2003, 42, 3693-3696.

135 498.V. Darcos, K. Griffith, X. Sallenave, J.-P. Desvergne, C. GuyardDuhayon, B. Hasenknopf and D. M. Bassani, Photochem. Photobiol. Sci., 2003, 2, 1152-1161.

499.B. Grosch, C. N. Orlebar, E. Herdtweck, M. Kaneda, T. Wada, Y. Inoue and T. Bach, Chem. Eur. J., 2004, 10, 2179-2189.

140 500.T. Aechtner, M. Dressel and T. Bach, Angew. Chem. Int. Ed., 2004, 43, 5849-5851.

501.A. Bauer, F. Westkämper, S. Grimme and T. Bach, Nature, 2005, 436, 1139-1140.

502.J.-i. Mizoguchi, Y. Kawanami, T. Wada, K. Kodama, K. Anzai, T. 145 Yanagi and Y. Inoue, Org. Lett., 2006, 8, 6051-6054. 
503.C. Müller, A. Bauer and T. Bach, Angew. Chem. Int. Ed., 2009, 48, $6640-6642$

504.K. A. B. Austin, E. Herdtweck and T. Bach, Angew. Chem. Int. Ed., 2011, 50, 8416-8419.

5 505.C. Müller, A. Bauer, M. M. Maturi, M. C. Cuquerella, M. A. Miranda and T. Bach, J. Am. Chem. Soc., 2011, 133, 16689-16697.

506.D. Uraguchi, K. Koshimoto and T. Ooi, J. Am. Chem. Soc., 2008, 130 10878-10879.

507.D. Uraguchi, K. Koshimoto, S. Miyake and T. Ooi, Angew. Chem. Int. Ed., 2010, 49, 5567-5569.

508.D. Uraguchi, K. Koshimoto and T. Ooi, J. Am. Chem. Soc., 2012, 134, 6972-6975.

509. Y. A. Cheng, T. Chen, C. K. Tan, J. J. Heng and Y. Y. Yeung, J. Am. Chem. Soc., 2012, 134, 16492-16495.

15 510.V. Rauniyar, A. D. Lackner, G. L. Hamilton and F. D. Toste, Science, 2010, 334, 1681-1684.

511.R. J. Phipps, K. Hiramatsu and F. D. Toste, J. Am. Chem. Soc., 2012, 134, 8376-8379.

512.C. K. De, E. G. Klauber and D. Seidel, J. Am. Chem. Soc., 2009, 131, 17060-17061.

513.N. Mittal, D. X. Sun and D. Seidel, Org. Lett., 2012, 14, 3084-3087.

514.E. G. Klauber, C. K. De, T. K. Shah and D. Seidel, J. Am. Chem. Soc., 2010, 132, 13624-13626.

515.C. K. De and D. Seidel, J. Am. Chem. Soc., 2011, 133, 14538-14541.

${ }_{25} 516$. C. K. De, N. Mittal and D. Seidel, J. Am. Chem. Soc., 2011, 133, 16802-16805.

517.C. Min, N. Mittal, C. K. De and D. Seidel, Chem. Commun., 2012, 48, 10853-10855.

518.J. W. Lee, H. Yan, H. B. Jang, H. K. Kim, S.-W. Park, S. Lee, D. Y.

30 Chi and C. E. Song, Angew. Chem. Int. Ed., 2009, 48, 7683-7686.

519.H. Yan, H. B. Jang, J.-W. Lee, H. K. Kim, S. W. Lee, J. W. Yang and C. E. Song, Angew. Chem. Int. Ed., 2010, 49, 8915-8917.

520.K. Sakthivel, W. Notz, T. Bui and C. F. Barbas III, J. Am. Chem. Soc., 2001, 123, 5260-5267.

35 521.S. Bahmanyar and K. N. Houk, J. Am. Chem. Soc., 2001, 123, $11273-$ 11283.

522.L. Hoang, S. Bahmanyar, K. N. Houk and B. List, J. Am. Chem. Soc., 2003, 125, 16-17.

523.C. Allemann, R. Gordillo, F. R. Clemente, P. H. Y. Cheong and K. N.

40 Houk, Acc. Chem. Res., 2004, 37, 558-569.

524.C. F. Barbas, Angew. Chem. Int. Ed., 2008, 47, 42-47.

525.R. K. Kunz and D. W. C. MacMillan, J. Am. Chem. Soc., 2005, 127, 3240-3241.

526.R. R. Shaikh, A. Mazzanti, M. Petrini, G. Bartoli and P. Melchiorre, Angew. Chem. Int. Ed., 2008, 47, 8707-8710.

527.T. Mandal and C.-G. Zhao, Angew. Chem. Int. Ed., 2008, 47, 77147717.

528.S. Muramulla and C.-G. Zhao, Tetrahedron Lett., 2011, 52, 39053908.

50 529.D. Sinha, T. Mandal, S. Gogoi, J. J. Goldman and J. C. G. Zhao, Chinese J. Chem., 2012, 30, 2624-2630.

530.D.-Q. Xu, H.-D. Yue, S.-P. Luo, A.-B. Xia, S. Zhang and Z.-Y. Xu, Org. Biomol.Chem., 2008, 6, 2054-2057.

531.A.-B. Xia, D.-Q. Xu, S.-P. Luo, J.-R. Jiang, J. Tang, Y.-F. Wang and Z.-Y. Xu, Chem. Eur. J., 2010, 16, 801-804.

532.Z.-B. Li, S.-P. Luo, Y. Guo, A.-B. Xia and D.-Q. Xu, Org. Biomol.Chem., 2010, 8, 2505-2508.

533.S. Mayer and B. List, Angew. Chem. Int. Ed., 2006, 45, 4193-4195.

534.X. Wang and B. List, Angew. Chem. Int. Ed., 2008, 47, 1119-1122.

60 535.N. J. A. Martin and B. List, J. Am. Chem. Soc., 2006, 128, 1336813369.

536.Y. Wang, T. Y. Yu, H. B. Zhang, Y. C. Luo and P. F. Xu, Angew. Chem. Int. Ed., 2012, 51, 12339-12342.

537.For example of enamine catalysts that benefit from the presence of acid addtives see references 537-538: S. Bertelsen, N. Halland, S. Bachmann, M. Marigo, A. Braunton and K. A. Jørgensen, Chem. Commun., 2005, 4821-4823.

538.T. J. Peelen, Y. Chi and S. H. Gellman, J. Am. Chem. Soc., 2005, 127, 11598-11599.

70 539.Y. Zhou and Z. Shan, J. Org. Chem., 2006, 71, 9510-9512.

540.Ö. Reis, S. Eymur, B. Reis and A. S. Demir, Chem. Commun., 2009, $1088-1090$
541.A. S. Demir and S. Eymur, Tetrahedron: Asymmetry, 2010, 21, 112115 .

75 542.X. Companyó, G. Valero, L. Crovetto, A. Moyano and R. Rios, Chem. Eur. J., 2009, 15, 6564-6568.

543.N. El-Hamdouni, X. Companyó, R. Rios and A. Moyano, Chem. Eur. $J ., 2010,1142-1148$.

544.S. L. Poe, A. R. Bogdan, B. P. Mason, J. L. Steinbacher, S. M. Opalka and D. T. McQuade, J. Org. Chem., 2009, 74, 1574-1580.

545.M. Bella, D. M. S. Schietroma, P. P. Cusella, T. Gasperi and V. Visca, Chem. Commun., 2009, 597-599.

546.G. Ma, A. Bartoszewicz, I. Ibrahem and A. Córdova, Adv. Synth. Catal., 2011, 353, 3114-3122.

85 547.M. L. Clarke and J. A. Fuentes, Angew. Chem. Int. Ed., 2007, 46, $930-$ 933.

548.J. A. Fuentes, T. Lebl, A. M. Z. Slawin and M. L. Clarke, Chem. Sci., 2011, 2, 1997-2005.

549.S. Bertelsen, M. Marigo, S. Brandes, P. Dinér and K. A. Jørgensen, $J$. Am. Chem. Soc., 2006, 128, 12973-12980.

550.G. Bergonzini, S. Vera and P. Melchiorre, Angew. Chem. Int. Ed., 2010, 49, 9685-9688.

551.Z. Shen, J. Ma, Y. Liu, C. Jiao, M. Li and Y. Zhang, Chirality, 2005, 17, 556-558.

95 552.J. Huang, X. Zhang and D. W. Armstrong, Angew. Chem. Int. Ed., 2007, 46, 9073-9077.

553.K. Liu, D. Häussinger and W.-D. Woggon, Synlett, 2007, 14, 22982300.

554.A.-B. Xia, D.-Q. Xu, C. Wu, L. Zhao and Z.-Y. Wu, Chem. Eur. J., 2012, 18, 1055-1059.

555.D. Q. Xu, S. P. Luo, Y. F. Wang, A. B. Xia, H. D. Yue, L. P. Wang and Z. Y. Xu, Chem. Commun., 2007, 4393-4395.

556.B. M. Nugent, R. A. Yoder and J. N. Johnston, J. Am. Chem. Soc., 2004, 126, 3418-3419.

105 557.A. Singh, R. A. Yoder, B. Shen and J. N. Johnston, J. Am. Chem. Soc., 2007, 129, 3466-3467.

558.M. C. Dobish and J. N. Johnston, J. Am. Chem. Soc., 2012, 134, 6068 6071.

559. For reviews on combined acid catalysis see references 559-560: $\mathrm{H}$.

110 Ishibashi, K. Ishihara and H. Yamamoto, Chem. Rec., 2002, 2, 177188.

560.H. Yamamoto and K. Futatsugi, Angew. Chem. Int. Ed., 2005, 44, 1924-1942.

561.M. Hatano, T. Mizuno, A. Izumiseki, R. Usami, T. Asai, M. Akakura 115 and K. Ishihara, Angew. Chem. Int. Ed., 2011, 50, 12189-12192.

562.M. Hatano and K. Ishihara, Chem. Commun., 2012, 48, 4273-4283.

563.D. Uraguchi, Y. Ueki and T. Ooi, Science, 2009, 326, 120-123.

564.H. Zhou, Z. Li, Z. Wang, T. Wang, L. Xu, Y. He, Q.-H. Fan, J. Pan, L. Gu and A. S. C. Chan, Angew. Chem. Int. Ed., 2008, 47, 8464-8467.

120 565.Z. J. Wang, H. F. Zhou, T. L. Wang, Y. M. He and Q. H. Fan, Green Chem., 2009, 11, 767-769.

566.M. Arthuis, R. Beaud, V. Gandon and E. Roulland, Angew. Chem. Int. Ed., 2012, 51, 10510-10514.

567.D. Chen, M. Schmitkamp, G. Franciò, J. Klankermayer and W. 125 Leitner, Angew. Chem. Int. Ed., 2008, 47, 7339-7341.

568.S. Z. Luo, L. Zhang and J. P. Cheng, Chem. Eur. J., 2009, 4, 1184 1195.

569.For seminal examples of asymmetric catalysts that may involve chirality transfer from a non-coordinated chiral anion to an achiral

130 metal complex see references 569-572: H. Alper and N. Hamel, J. Am. Chem. Soc., 1990, 112, 2803-2804.

570.D. B. Llewellyn, D. Adamson and B. A. Arndtsen, Org. Lett., 2000, 2, 4165-4168.

571.M. C. Lacasse, C. Poulard and A. B. Charette, J. Am. Chem. Soc., 2005, 135 127, 12440-12441.

572.V. Komanduri and M. J. Krische, J. Am. Chem. Soc., 2006, 128, 16448-16449.

573.J. Lacour and V. Hebbe-Viton, Chem. Soc. Rev., 2003, 32, 373-382.

574.G. L. Hamilton, E. J. Kang, M. Mba and F. D. Toste, Science, 2007, 317, 496-499.

575.M. Barbazanges, M. Augé, J. Moussa, H. Amouri, C. Aubert, C. Desmarets, L. Fensterbank, V. Gandon, M. Malacria and C. Ollivier, Chem. Eur. J., 2011, 17, 13789-13794.

576.R. L. LaLonde, Z. J. Wang, M. Mba, A. D. Lackner and F. D. Toste, Angew. Chem. Int. Ed., 2010, 49, 598-601. 
577.R. L. LaLonde, B. D. Sherry, E. J. Kang and F. D. Toste, J. Am. Chem. Soc., 2007, 129, 2452-2453.

578.J. H. Kim, S.-W. Park, S. R. Park, S. Lee and E. J. Kang, Chem. Asian J., 2011, 6, 1982-1986.

${ }_{5}$ 579.K. Aikawa, M. Kojima and K. Mikami, Adv. Synth. Catal., 2010, 352, 3131-3135.

580.S. Liao and B. List, Angew. Chem. Int. Ed., 2010, 49, 628-631.

581.S. Liao and B. List, Adv. Synth. Catal., 2012, 354, 2363-2367.

582.L. Kurti, M. M. Blewett and E. J. Corey, Org. Lett., 2009, 11, 4592-

104595 .

583. For reviews dealing with the chiral counteranion strategy applied to catalysis and possible mode of action see references 52, 53, 272 and 583: J. Lacour and D. Moraleda, Chem. Commun., 2009, 7073-7089.

584.B. G. Zhao, H. F. Du and Y. A. Shi, J. Org. Chem., 2009, 74, 8392158395.

585.R. Yazaki, N. Kumagai and M. Shibasaki, J. Am. Chem. Soc., 2010, 132, $10275-10277$.

586.D. Chen, B. Sundararaju, R. Krause, J. Klankermayer, P. H. Dixneuf and W. Leitner, Chem CatChem, 2010, 2, 55-57.

20 587.G. X. Jiang and B. List, Chem. Commun., 2011, 47, 10022-10024.

588.G. Jiang, R. Halder, Y. Fang and B. List, Angew. Chem. Int. Ed., 2011, 50, 9752-9755.

589.B. Guo, G. Schwarzwalder and J. T. Njardarson, Angew. Chem. Int. Ed., 2012, 51, 5675-5678.

25 590.Z. Chai and T. J. Rainey, J. Am. Chem. Soc., 2012, 134, 3615-3618.

591.J. R. Zbieg, E. Yamaguchi, E. L. McInturff and M. J. Krische, Science, 2012, 336, 324-327.

592.S. Mukherjee and B. List, J. Am. Chem. Soc., 2007, 129, 11336-11337.

593.G. Jiang and B. List, Angew. Chem. Int. Ed., 2011, 50, 9471-9474.

30 594.W.-H. Hu, X.-F. Xu, J. Zhou, W.-J. Liu, H.-X. Huang, J. Hu, L.-P. Yang and L.-Z. Gong, J. Am. Chem. Soc., 2008, 130, 7782-7783.

595.X. F. Xu, J. Zhou, L. P. Yang and W.-H. Hu, Chem. Commun., 2008, 6564-6566.

596. J. Jiang, H.-D. Xu, J.-B. Xi, B.-Y. Ren, F.-P. Lv, X. Guo, L.-Q. Jiang,

35 Z.-Y. Zhang and W.-H. Hu, J. Am. Chem. Soc., 2011, 133, 8428-8431.

597.M. Rueping, A. P. Antonchick and C. Brinkmann, Angew. Chem. Int. Ed., 2007, 46, 6903-6906.

598.Y. D. Lu, T. C. Johnstone and B. A. Arndtsen, J. Am. Chem. Soc., 2009, 131, 11284-11285.

40 599.S. Zhou, S. Fleischer, K. Junge and M. Beller, Angew. Chem. Int. Ed., 2011, 50, 5120-5124.

600.C. Q. Li, C. Wang, B. Villa-Marcos and J. L. Xiao, J. Am. Chem. Soc., 2008, 130, 14450-14451.

601.M. Rueping and R. M. Koenigs, Chem. Commun., 2011, 47, 304-306.

45 602.Enantioselective Povarov reactions can also be achieved by means of chiral Brønsted acid catalysts see references 602-603: T. Akiyama, H. Morita and K. Fuchibe, J. Am. Chem. Soc., 2006, 128, 13070-13071.

603.H. Liu, G. Dagousset, G. Masson, P. Retailleau and J. P. Zhu, J. Am. Chem. Soc., 2009, 131, 4598-4599.

50 604.M. T. Reetz, Angew. Chem. Int. Ed., 2008, 47, 2556-2588.

605.K. N. Houk and P. H.-Y. Cheong, Nature, 2008, 455, 309-313. 